U.S. DEPARTMENT OF COMMERCE / National Bureau of Standards

\title{
Tables of Industrial Gas Container Contents and Density for Oxygen, Argon, Nitrogen, Helium, and Hydrogen
}

Ben A. Younglove Neil A. Olien 
he National Bureau of Standards ' was established by an act of Congress on March 3, 1901. The

Bureau's overall goal is to strengthen and advance the nation's science and technology and facilitate their effective application for public benefit. To this end, the Bureau conducts research and provides: (1) a basis for the nation's physical measurement system, (2) scientific and technological services for industry and government, (3) a technical basis for equity in trade, and (4) technical services to promote public safety. The Bureau's technical work is performed by the National Measurement Laboratory, the National Engineering Laboratory, the Institute for Computer Sciences and Technology, and the Center for Materials Science.

\section{The National Measurement Laboratory}

Provides the national system of physical and chemical measurement; coordinates the system with measurement systems of other nations and furnishes essential services leading to accurate and uniform physical and chemical measurement throughout the Nation's scientific community, industry, and commerce; provides advisory and research services to other Government agencies; conducts physical and chemical research; develops, produces, and distributes Standard Reference Materials; and provides calibration services. The Laboratory consists of the following centers:
- Basic Standards ${ }^{2}$

- Radiation Research

- Chemical Physics

- Analytical Chemistry

\section{The National Engineering Laboratory}

Provides technology and technical services to the public and private sectors to address national needs and to solve national problems; conducts research in engineering and applied science in support of these efforts; builds and maintains competence in the necessary disciplines required to carry out this research and technical service; develops engineering data and measurement capabilities; provides engineering measurement traceability services; develops test methods and proposes engineering standards and code changes; develops and proposes new engineering practices; and develops and improves mechanisms to transfer results of its research to the ultimate user. The Laboratory consists of the following centers:
- Applied Mathematics

- Electronics and Electrical Engineering ${ }^{2}$

- Manufacturing Engineering

- Building Technology

- Fire Research

- Chemical Engineering?

\section{The Institute for Computer Sciences and Technology}

Conducts research and provides scientific and technical services to aid Federal agencies in the selection, acquisition, application, and use of computer technology to improve effectiveness and economy in Government operations in accordance with Public Law 89-306 (40 U.S.C. 759), relevant Executive Orders, and other directives; carries out this mission by managing the Federal Information Processing Standards Program, developing Federal ADP standards guidelines, and managing Federal participation in ADP voluntary standardization activities; provides scientific and technological advisory services and assistance to Federal agencies; and provides the technical foundation for computer-related policies of the Federal Government. The Institute consists of the following centers:
- Programming Science and Technology

- Computer Systems Engineering

\section{The Center for Materials Science}

Conducts research and provides measurements, data, standards, reference materials, quantitative understanding and other technical information fundamental to the processing, structure, properties and performance of materials; addresses the scientific basis for new advanced materials technologies; plans research around cross-country scientific themes such as nondestructive evaluation and phase diagram development: oversees Bureau-wide technical programs in nuclear reactor radiation research and nondestructive evaluation; and broadly disseminates generic technical information resulting from its programs. The Center consists of the following Divisions:
- Inorganic Materials

- Fracture and Deformation ${ }^{3}$

- Polymers

- Metallurgy

- Reactor Radiation 


\section{Tables of Industrial Gas Container Contents and Density for Oxygen, Argon, Nitrogen, Helium, and Hydrogen}

Ben A. Younglove

Neil A. Olien

Chemical Engineering Science Division

National Engineering Laboratory

National Bureau of Standards

U.S. Department of Commerce

Boulder, Colorado 80303

Sponsored by

Compressed Gas Association

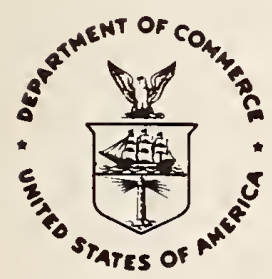

U.S. DEPARTMENT OF COMMERCE, Malcolm Baldrige, Secretary

NATIONAL BUREAU OF STANDARDS, Ernest Ambler, Director 
National Bureau of Standards Technical Note 1079

Natl. Bur. Stand. (U.S.), Tech Note 1079, 200 pages (June 1985) CODEN: NBTNAE

\section{U.S. GOVERNMENT PRINTING OFFICE}

WASHINGTON: 1985

For sale by the Superintendent of Documents, U.S. Government Printing Office, Washington, DC 20402 
1. Introduction . . . . . . . . . . . . . . . . . . . 1

2. Scope .. . . . . . . . . . . . . . . . 2

2.1 Contents Tables . . . . . . . . . . . . . . 2

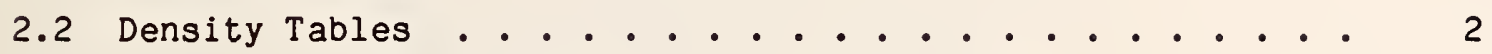

2.3 Standard Conditions . . . . . . . . . . . . . 3

3. Using the Tables . . . . . . . . . . . . . . . . . 3

3.1 Measurements and Data Required . . . . . . . . . . 3

3.2 Calculations .. . . . . . . . . . . . . . 3

3.3 Interpolation ................... . . . 4

4. Examples . . . . . . . . . . . . . . . . . 4

4.1 Cylinder Volume Calculations - Example 1 . . . . . . . 4

4.2 Cylinder Volune Calculation - Example 2 (with pressure interpolation) .................. 4

4.3 Tube Trailer Volume Calculation - Example 3 . . . . . . 5

4.4 Cylinder Mass Calculation - Example 4 (International System of Units) . . . . . . . . . . . . 5

5. Acknowledgments . . . . . . . . . . . . . . . 6

6. References . . . . . . . . . . . . . . . . 7

7. Conversion Table . . . . . . . . . . . . . . . 8

8. Cylinder Contents Tables in Engineering Units . . . . . . . . 9

8.1 Oxygen Contents Table (SCF/cu ft) . . . . . . . . 10

8.2 Argon Contents Table (SCF/cu ft) . . . . . . . . . 26

8.3 Nitrogen Contents Table (SCF/cu ft)........... 42

8.4 Helium Contents Table (SCF/cu ft) . . . . . . . . 58

8.5 Hydrogen Contents Table (SCF/cu ft) . . . . . . . . 74

9. Density Tables in SI Units . . . . . . . . . . . . . 90

9.1 Oxygen Density $\left(\mathrm{kg} / \mathrm{m}^{3}\right)$. . . . . . . . . . . . . 91

9.2 Argon Density $\left(\mathrm{kg} / \mathrm{m}^{3}\right)$. . . . . . . . . . . . . 112

9.3 Nitrogen Density $\left(\mathrm{kg} / \mathrm{m}^{3}\right)$. . . . . . . . . . . . 133

9.4 Helium Density $\left(\mathrm{kg} / \mathrm{m}^{3}\right)$. . . . . . . . . . . 154

9.5 Hydrogen Density $\left(\mathrm{kg} / \mathrm{m}^{3}\right)$. . . . . . . . . . . 175 

TABLES OF INDUSTRIAL GAS CONTAINER CONTENTS AND DENSITY FOR

OXYGEN, ARGON, NITROGEN, HELIUM, AND HYDROGEN

\author{
by \\ Ben A. Younglove and Neil A. Olien \\ Chemical Engineering Science Division \\ National Engineering Laboratory \\ National Bureau of Standards \\ Boulder, Colorado 80303
}

\begin{abstract}
Custody transfer tables are presented for oxygen, argon, nitrogen, helium, and hydrogen. The tables are based on standard reference data previously compiled by the National Bureau of Standards. Two sets of tables are provided for each fluid. Tables in engineering units cover the range -40 to $130^{\circ} \mathrm{F}$ with pressures from 100 to 10,000 psig. Tables in SI units (density versus pressure and temperature) cover the range 200 to $370 \mathrm{~K}$ with pressures from 0.5 to $70 \mathrm{MPa}$. The tables in engineering units are designed to provide a means of determining the volume of gas at standard conditions contained in a tank given the volume of the tank and the pressure and temperature of the gas within the tank. The publication also includes four examples of use of the tables in calculating tank quantities.
\end{abstract}

Key words: argon; custody transfer; gas density; gas volume; helium; hydrogen; nitrogen; oxygen.

1. Introduction

Industrial gases are important commodities in the Chemical Processes Industry as well as in other segments of the U. S. economy. Custody transfer of these gases usually takes place at high pressure and ambient temperatures with a wide variation in the latter depending on location and season. Normal custody transfer is based upon the volume the gas would occupy at standard conditions (standard conditions are defined here as $294.26 \mathrm{~K}\left(70.0^{\circ} \mathrm{F}\right)^{1}$ and $0.101325 \mathrm{MPa}$ $\left.\left(14.696 \mathrm{lb} / \mathrm{in}^{2}\right)\right)^{1}$. The tables presented are designed to provide a relatively easy means of determining the volume of gas at standard conditions contained in a tank given the volume of the tank and the pressure and temperature of the gas within the tank. Tables are provided for each of the five fluids: oxygen, argon, nitrogen, helium, and hydrogen. Also included for each of the five

1 Departing from usual NBS practice, the International System of Units (SI) were not used exclusively in this publication in order to meet the needs of the sponsoring agency, the Compressed Gas Association. 
fluids are tables in SI units (the International System of Units) of density as a function of pressure and temperature.

The National Bureau of Standards has a long history of accurate determination of the thermophysical properties of fluids and in the critical evaluation and correlation of the properties of a wide variety of pure fluids and mixtures. Much of the latter work was performed under the sponsorship of other government agencies. The work from which these tables are derived is a good example. The helium and hydrogen tables were prepared from a computer program called "Fluids Pack" (McCarty [1]) at NBS, Chemical Engineering Science Division for the Johnson Space Center of the National Aeronautics and Space Administration. The tables for the other three fluids were derived from tables of thermophysical properties and accompanying computer programs prepared under the sponsorship of the NBS-Office of Standard Reference Data (Younglove $[2,3]$ ). The computer codes are available from the office of Standard Reference Data [4] for those desiring copies or requiring data over wider ranges. The tables presented here were prepared under the sponsorship of the Compressed Gas Association (CGA).

\section{Scope}

Two tables are provided for each of the five fluids. The first gives contents in standard cubic feet/cubic feet (SCF/cu $\mathrm{ft}$ ) as a function of temperature and pressure ( ${ }^{\circ} \mathrm{F}$ and $\mathrm{psig}$ ) and the second gives density in $\mathrm{kg} / \mathrm{m}^{3}$ as a function of temperature and pressure ( $K$ and $M P a)$.

\subsection{Contents Tables}

The contents tables give the volume that a cubic foot of gas would occupy at standard conditions if a cubic foot at a given temperature and pressure were expanded to standard conditions. The tables provide these values in the range -40 to $130^{\circ} \mathrm{F}$ at $2^{\circ} \mathrm{F}$ increments and at 100 psig increments in the range from 100 to 10,000 psig. Gauge pressure (psig) is normally taken to be the gauge reading at sea level and one atmosphere $(14.696 \mathrm{psig})$. These tables use this convention.

\subsection{Density Tables}

The density tables give the density as a function of pressure and temperature in SI units. These tables may be used when it is necessary to determine the mass of gas in a container at a given temperature and pressure. The tables provide density in $\mathrm{kg} / \mathrm{m}^{3}$ over the range from 200 to $370 \mathrm{~K}$ at $2 \mathrm{~K}$ increments and at $0.5 \mathrm{MPa}$ increments in the range from 0.5 to $70 \mathrm{MPa}$. 


\subsection{Standard Conditions}

The standard conditions used in these tables are those specified by the Compressed Gas Association [5]. These are defined as $70^{\circ} \mathrm{F}(294.26 \mathrm{~K})$ and $14.696 \mathrm{lb} / \mathrm{in}^{2}(0.101325 \mathrm{MPa})$.

3. Using the Tables

3.1 Measurements and Data Required

The following data are needed to determine the amount of gas in the container:

Temperature - the temperature of the gas in the container or the temperature of the container itself must be determined.

Pressure - the pressure of the gas in the container must be determined.

Volume - the volume of the container must be known. This is usually provided by the manufacturer or supplier of the container and is determined by measuring the volume of water the tank holds.

\subsection{Calculations}

Once the temperature and pressure are determined for a particular gas, the rable is used to determine the quantity of that gas in the container. The use of the Contents Tables (engineering units) and Density Tables (SI units) are as follows:

Contents Table - use the following formula

$\mathrm{V}=\mathrm{C} \times \mathrm{V}_{\mathrm{T}} \quad$ where:

$V$ is the volume in cu $\mathrm{ft}$ which the gas in the tank would occupy at standard conditions $\left(70^{\circ} \mathrm{F}\right.$ and $\left.14.696 \mathrm{psia}\right)$, noted as SCF,

$C$ is the value obtained from the contents table at the temperature and pressure of the container, $V_{T}$ is the volume of the tank in cu $\mathrm{ft}$

Density Table - use the following formula

$M=D \times V_{T} \quad$ where:

$M$ is the mass of gas in the tank in $\mathrm{kg}$. ,

$D$ is the density value obtained from the table at the temperature and pressure of the container, $V_{T}$ is the water volume of the tank in $\mathrm{m}^{3}$. 


\subsection{Interpolation}

When gauges used to measure the temperature and pressure of the container are more accurate than the increments for temperature $\left( \pm 2^{\circ} \mathrm{F}\right.$ or $\left.\pm 2 \mathrm{~K}\right)$ or pressure $( \pm 100$ psig or $\pm 0.5 \mathrm{MPa})$ given in the tables, it is appropriate to interpolate between the next highest and next lowest values in the tables. Examples of interpolation will be given in Section 4.

\section{Examples}

For the sake of simplicity, nitrogen tables will be used in all of the examples, but the calculations are performed in exactly the same manner for all of the fluids.

4.1 Cylinder Volume Calculations - Example 1

A nitrogen cylinder has:

- Temperature of $68^{\circ} \mathrm{F}$

- Pressure of 2300 psig

- Water volume of 2675 cubic inches (please note we must have the volume in cubic feet, therefore $V_{T}=2675 / 1728^{2}=1.548 \mathrm{ft}^{3}$ ).

The formula from Section 3.2 is:

$$
\mathrm{V}=\mathrm{C} \times \mathrm{V}_{\mathrm{T}}
$$

from the table for $68^{\circ} \mathrm{F}$ and 2300 psig we obtain $\mathrm{C}=154.1$, therefore

$\mathrm{V}=154.1 \times 1.548$

$V=238.5 \mathrm{SCF}$

4.2 Cylinder Volume Calculation - Example 2 (with pressure interpolation)

A nitrogen cylinder has:

- Temperature of $64^{\circ} \mathrm{F}$

- Pressure of 1840 psig

- Water volume of $4.46 \mathrm{ft}^{3}$

The formula remains the same:

$$
\mathrm{V}=\mathrm{C} \times \mathrm{V}_{\mathrm{T}}
$$

${ }^{2}$ one cubic foot $=1728$ cubic inches. 
but we must interpolate to find $C$. This is done as follows: look up the values in the table at $64^{\circ} \mathrm{F}$ for 1800 and $1900 \mathrm{psig}$. The correct value of $\mathrm{C}$ will be the sum of the value at 1800 psig, plus $4 / 10$ of the difference between 1800 psig and 1900 psig, or as follows:

$$
\begin{aligned}
C & =124.0+4 / 10(130.4-124.0) \\
& =124.0+2.56 \\
& =126.6 \text { (rounding off to four digits) }
\end{aligned}
$$

The final calculation is:

$$
\begin{aligned}
& V=126.6 \times 4.46 \\
& V=564.6 \mathrm{sCF}
\end{aligned}
$$

4.3 Tube Trailer Volume Calculation - Example 3

A nitrogen tube trailer has:

- Temperature of $50^{\circ} \mathrm{F}$

- Pressure of 2470 psig

- 30 tubes each of which has a water volume of $15.50 \mathrm{ft}^{3}$

The interpolation is as follows:

$$
\begin{aligned}
& C=167.2+7 / 10(173.5-167.2) \\
& C=171.6
\end{aligned}
$$

where 173.5 and 167.2 are taken from the table at $50^{\circ} \mathrm{F}$ and $2500 \mathrm{psig}$ and 2400 psig, respectively.

The formula for calculating the contents of the tube trailer is:

$\mathrm{V}=\mathrm{C} \times \mathrm{V}_{\mathrm{T}} \times \mathrm{N}$; where $\mathrm{N}$ is the number of tubes

$V=171.6 \times 15.50 \times 30$

$V=79,749 \mathrm{SCF}$

4.4 Cylinder Mass Calculation - Example 4 (International System of Units)

A nitrogen cylinder has:

- Temperature of $300 \mathrm{~K}$

- Pressure of $10 \mathrm{MPa}$

- Water volume of $0.085 \mathrm{~m}^{3}$

The formula is:

$$
\begin{aligned}
& M=D \times V_{T} \\
& M=111.7 \times 0.085 \\
& M=9.495 \mathrm{~kg}
\end{aligned}
$$


5. Acknowledgments

We acknowledge the the Compressed Gas Association for their interest and support of this project. 
6. References

[1] McCarty, R. D. Interactive Fortran IV Computer Programs for the Thermodynamic and Transport Properties of Selected Cryogens (Fluids Pack). Nat. Bur. Stand. (U.S.) Tech. Note No. 1025; 1980 October. 112 p.

[2] Younglove, B. A. Thermophysical Properties of Fluids. 1. Argon, Ethylene, Parahydrogen, Nitrogen, Nitrogen Trifluoride, and Oxygen. J. Phys. Chem. Ref. Data 11, Supplement; 1982. 368 p.

[3] Younglove, B. A. Interactive Fortran Program to Calculate Thermophysical Properties of Six Fluids. Nat. Bur. Stand. (U.S.) Tech. Note 1048; 1982 June. $56 \mathrm{p}$.

[4] For magnetic tape, order NBS Standard Reference Data Base 5 for helium properties and Data Base 6 for the other fluids from the Office of Standard Reference Data, A320 Physics Building, National Bureau of Standards, Gaithersburg, MD 20899, for $\$ 200$ and $\$ 500$ respectively.

[5] The Compressed Gas Association has defined Standard Temperature and Pressure (STP) as $70^{\circ} \mathrm{F}$ and $14.696 \mathrm{lb} / \mathrm{in}^{2}$. See Metric Practice Guide for the Compressed Gas Industry, Compressed Gas Association Publication CGA $\mathrm{P}-11 ; 1980$.

7 
7. Conversion Table

To Convert

TEMPERATURE

PRESSURE

DENSITY

MOLECULAR WEIGHTS

Oxygen

Argon

Nitrogen

Helium

Hydrogen

31.9988

39.948

28.013

4.0026

2.01594

$$
\text { From }
$$

To

$\mathrm{K}$

K

${ }^{\circ} \mathrm{F}$

${ }^{\circ} \mathrm{F}$

${ }^{\circ} \mathrm{C}$

To Convert

From

$\mathrm{MPa}$

psi

atm

atm

$\mathrm{kg} / \mathrm{m}^{3}$

$1 \mathrm{~b} / \mathrm{et}^{3}$
To

psi

$\mathrm{MPa}$

$\mathrm{MPa}$

psi

$1 b / f^{3}$

$\mathrm{kg} / \mathrm{m}^{3}$
Use

$273.15+\left({ }^{\circ} F-32\right) / 1.8$

$273.15+{ }^{\circ} \mathrm{C}$

$32+1.8 * 0 \mathrm{C}$

$32+(K-273.15) * 1.8$

$K-273.15$

Multiply By

145.03775

0.006894757

0.101325

14.696

0.0624280

16.018463

Density at STP (70 F and 14.696 psia or $294.261 \mathrm{~K}$ and $0.101325 \mathrm{MPa}$ )

GAS

$\mathrm{kg} / \mathrm{m}^{3}$

$1 b / \mathrm{ft}^{3}$

Oxygen

Argon

Nitrogen

Helium

Hydrogen

$\begin{array}{ll}1.32615 & 0.082789 \\ 1.65555 & 0.103353 \\ 1.16058 & 0.072452 \\ 0.165686 & 0.0103434 \\ 0.083438 & 0.0052089\end{array}$


8.0

Cylinder Contents Tables in Engineering Units

9 
8.1

Oxygen Contents Table (SCF/cu $\mathrm{ft}$ ) 
OXYGEN CONTENTS TABLE (SCF/CU FT).

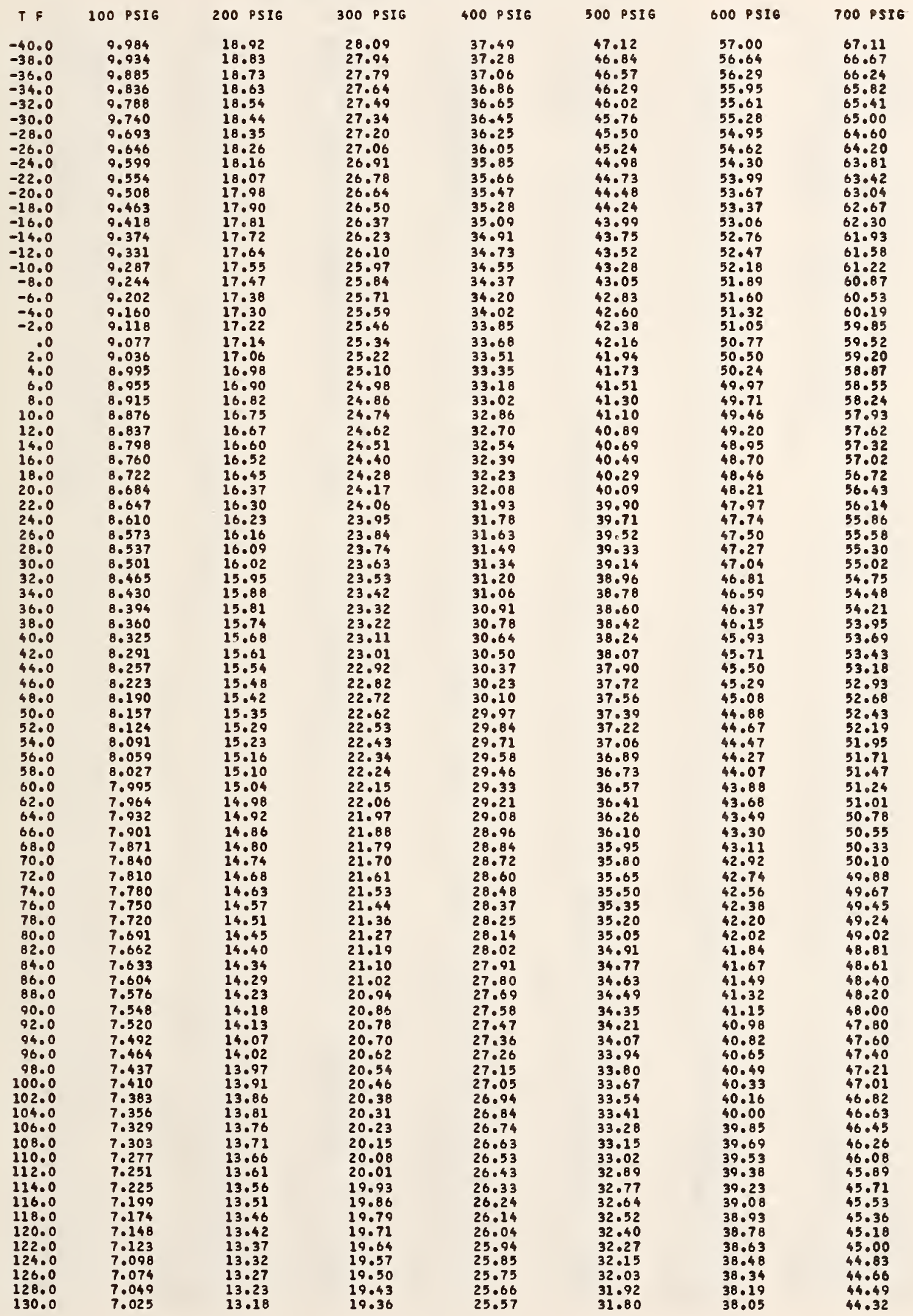


OXYGEN CONTENTS TABLE (SCF/CU FT).

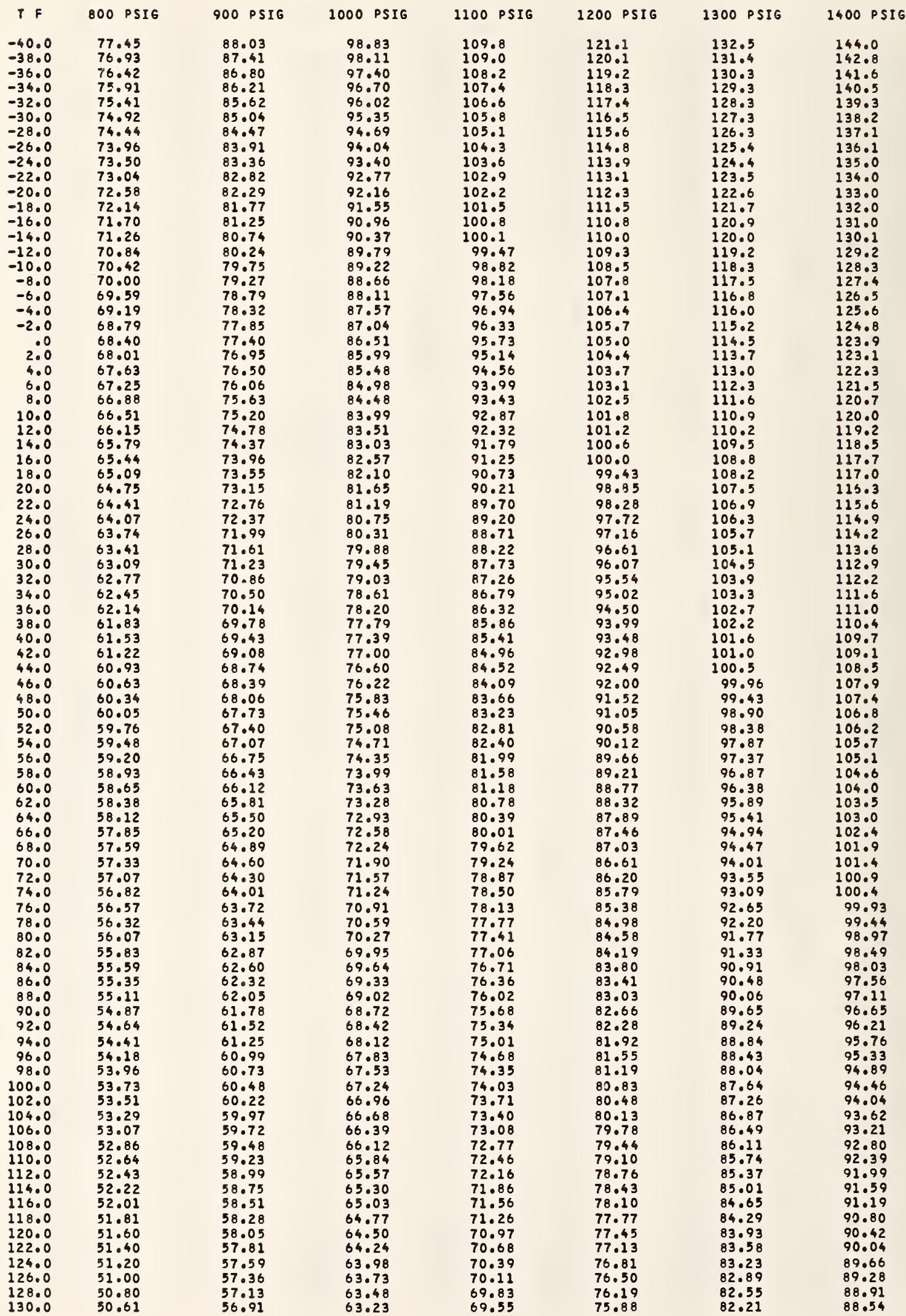


OXYGEN CONTENTS TABLE (SCFICU FT).

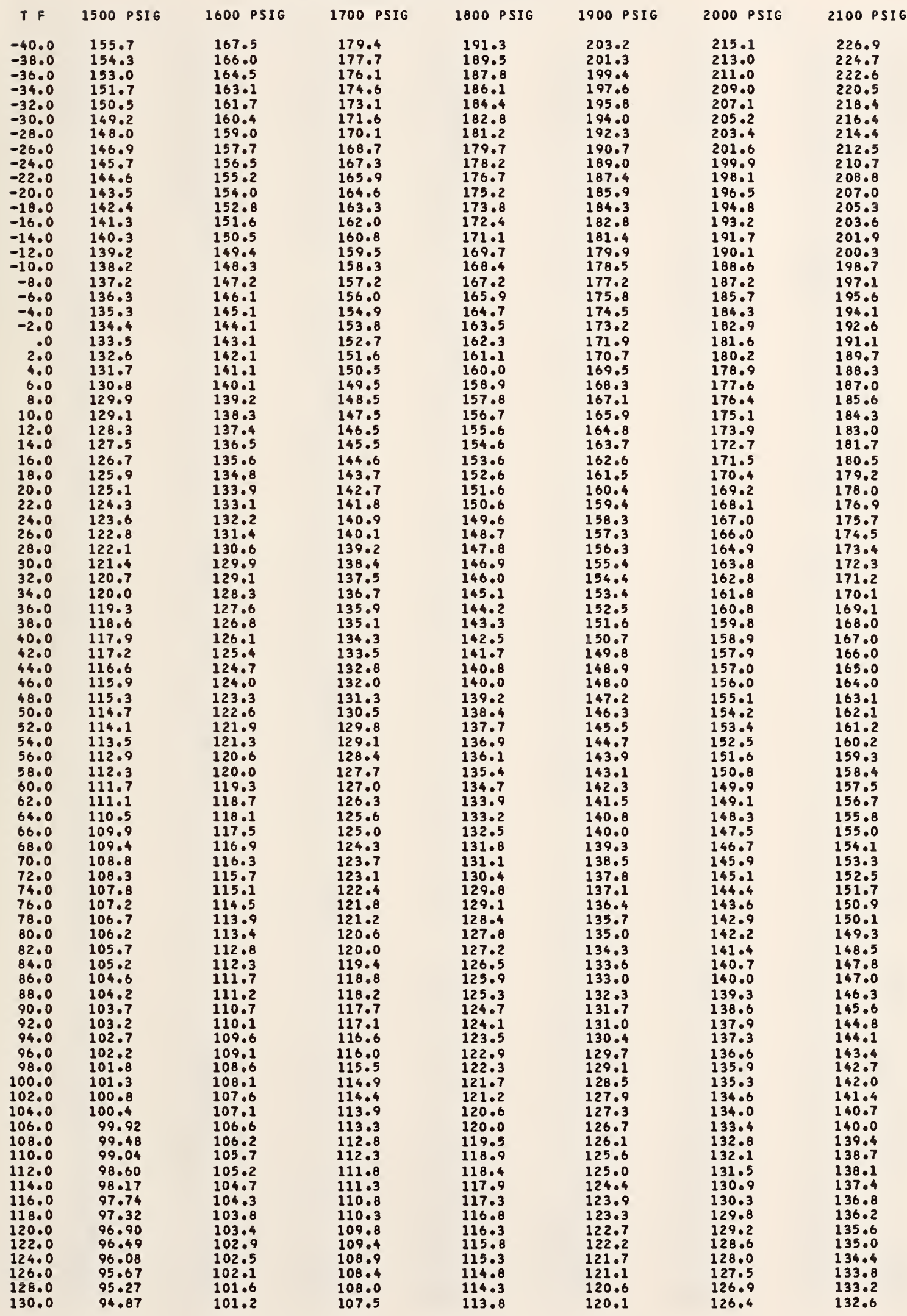


OXYGEN CONTENTS TABLE (SCF/CU FT).

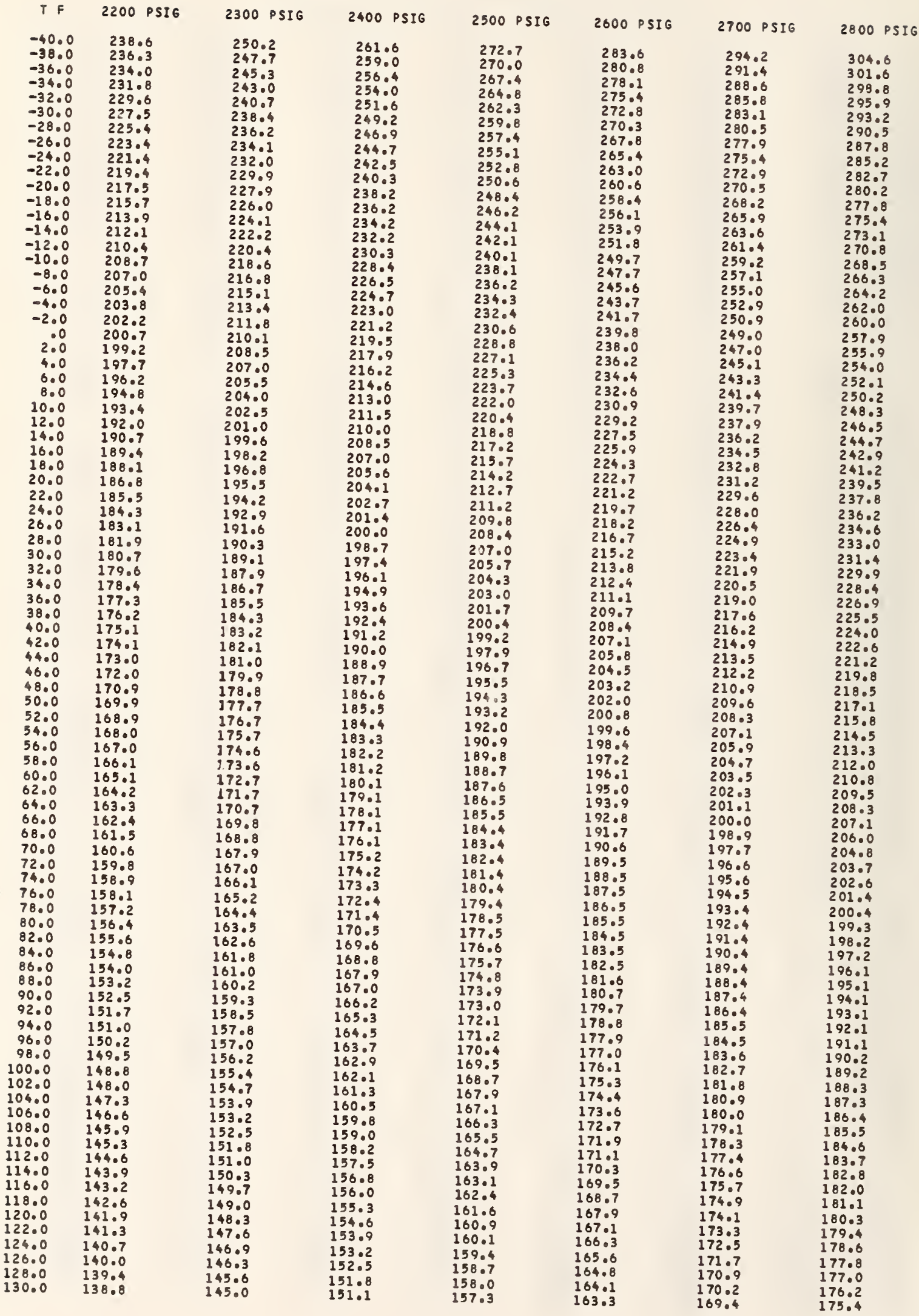


OXYGEN CONTENTS TABLE (SCFICU FTI.

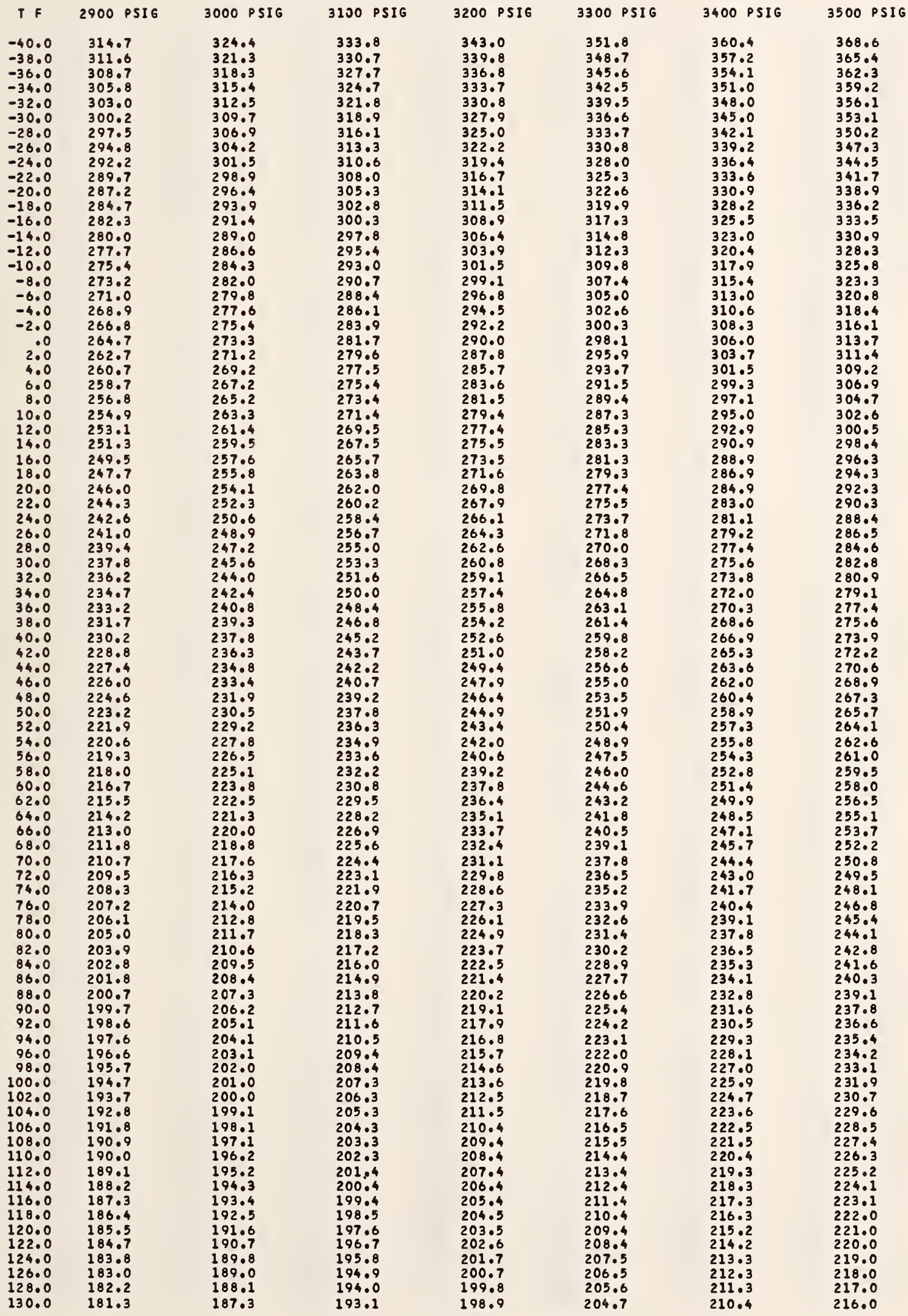


OXYGEN CONTENTS TABLE (SCF/CU FT).

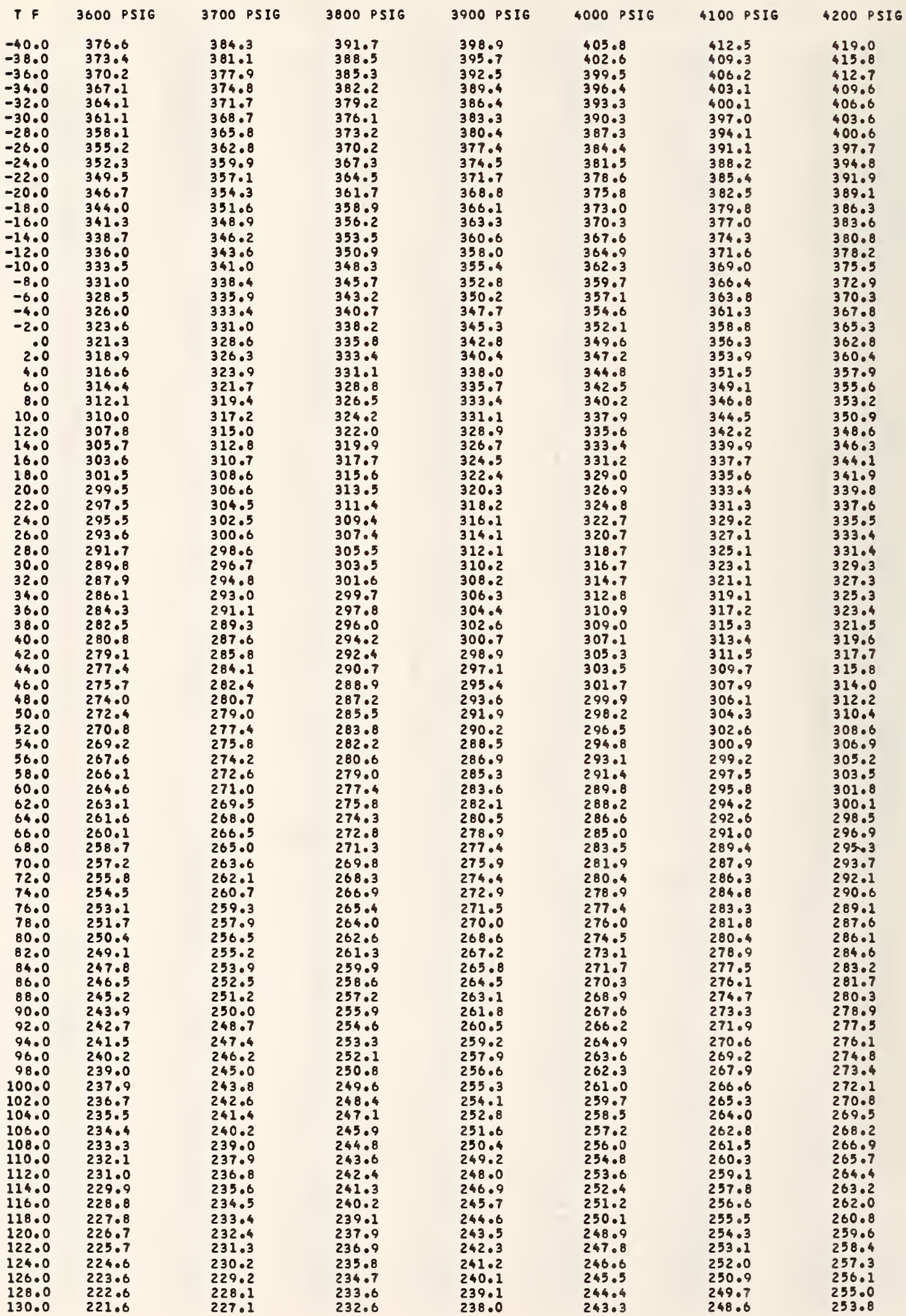


OXYGEN CONTENTS TABLE (SCF/CU FT).

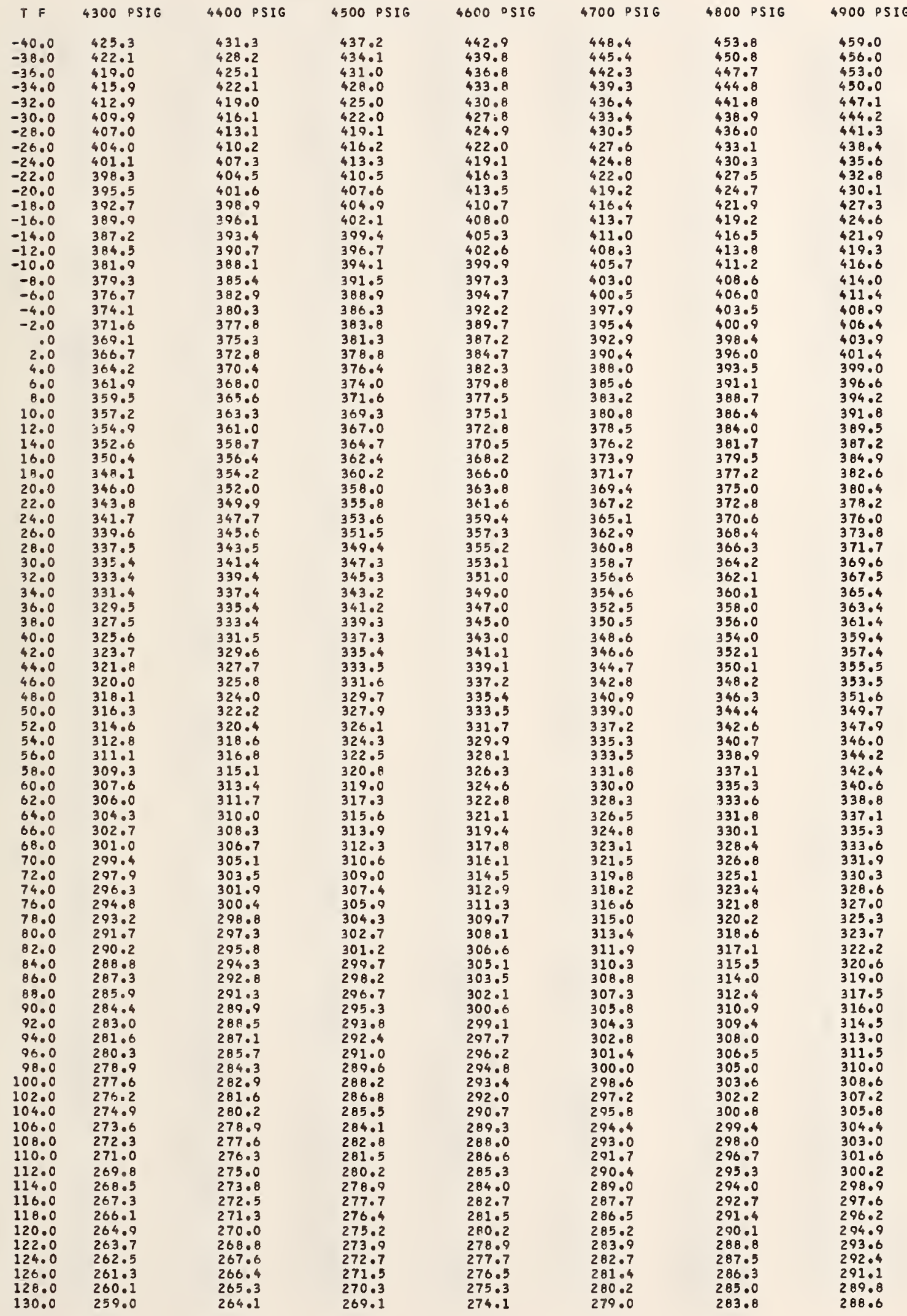


OXYGEN CONTENTS TABLE (SCF/CU FT).

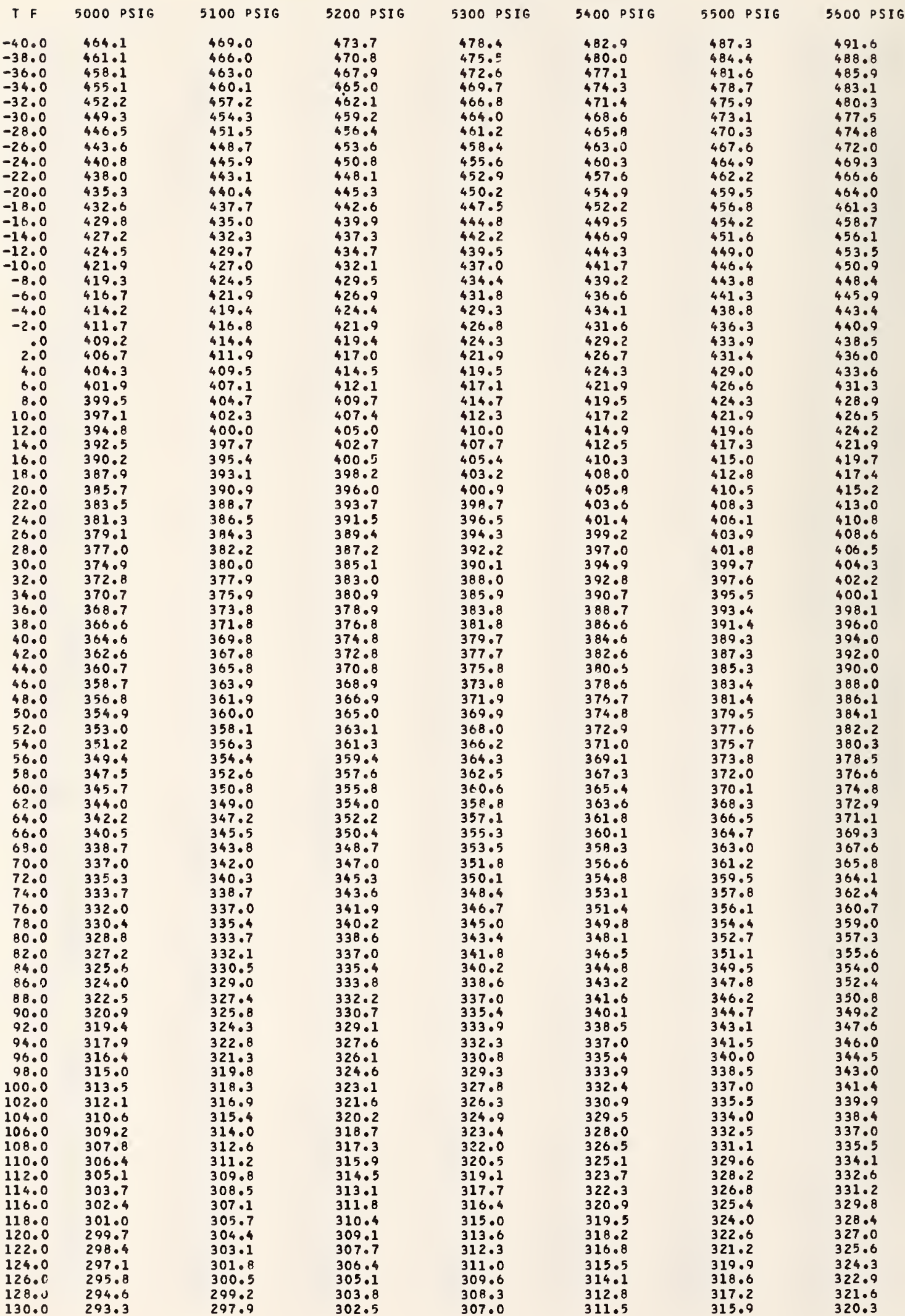


OXYGEN CONTENTS TABLE (SCFICU FT).

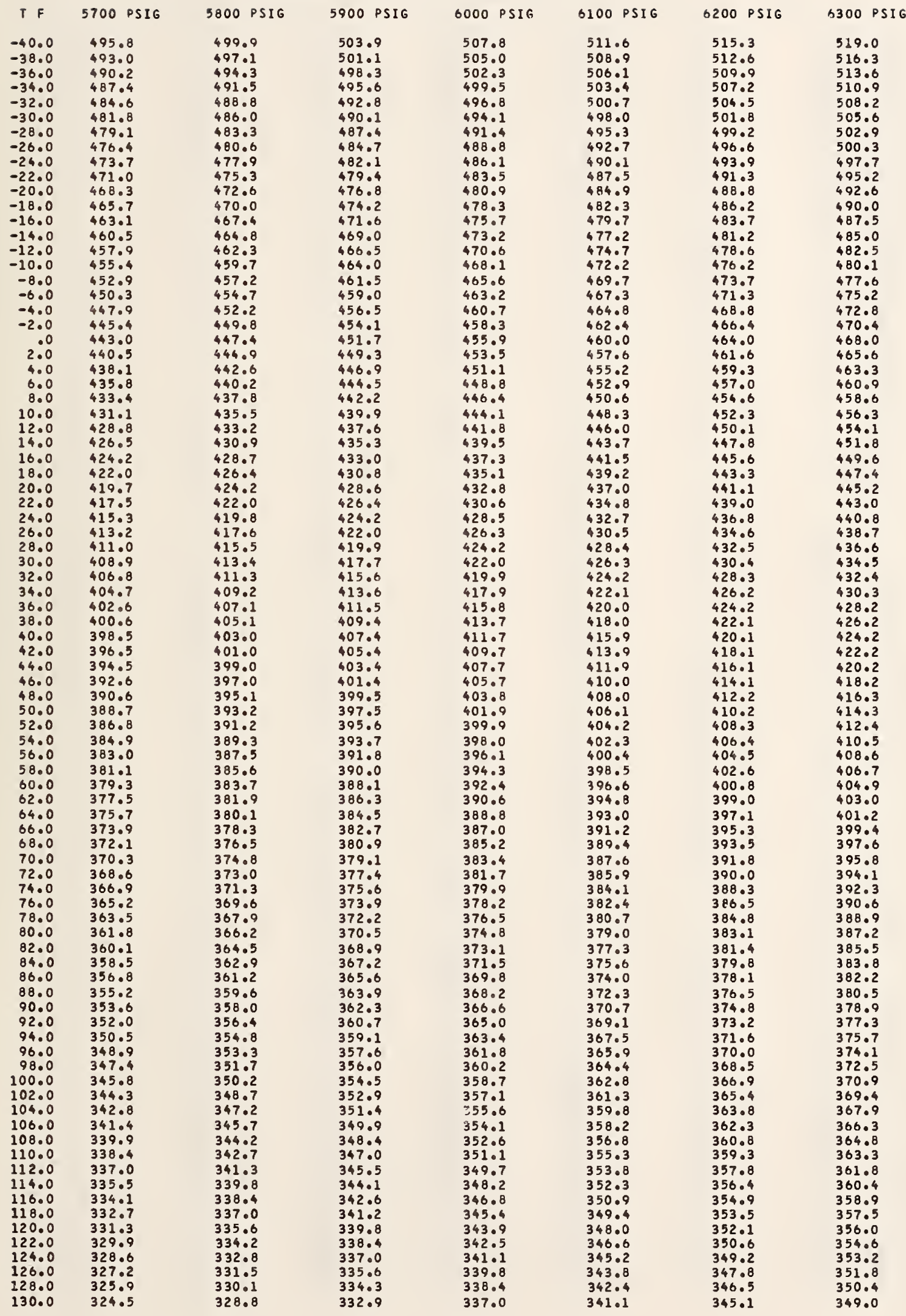


DXYGEN CONTENTS TABLE (SCF/CU FT).

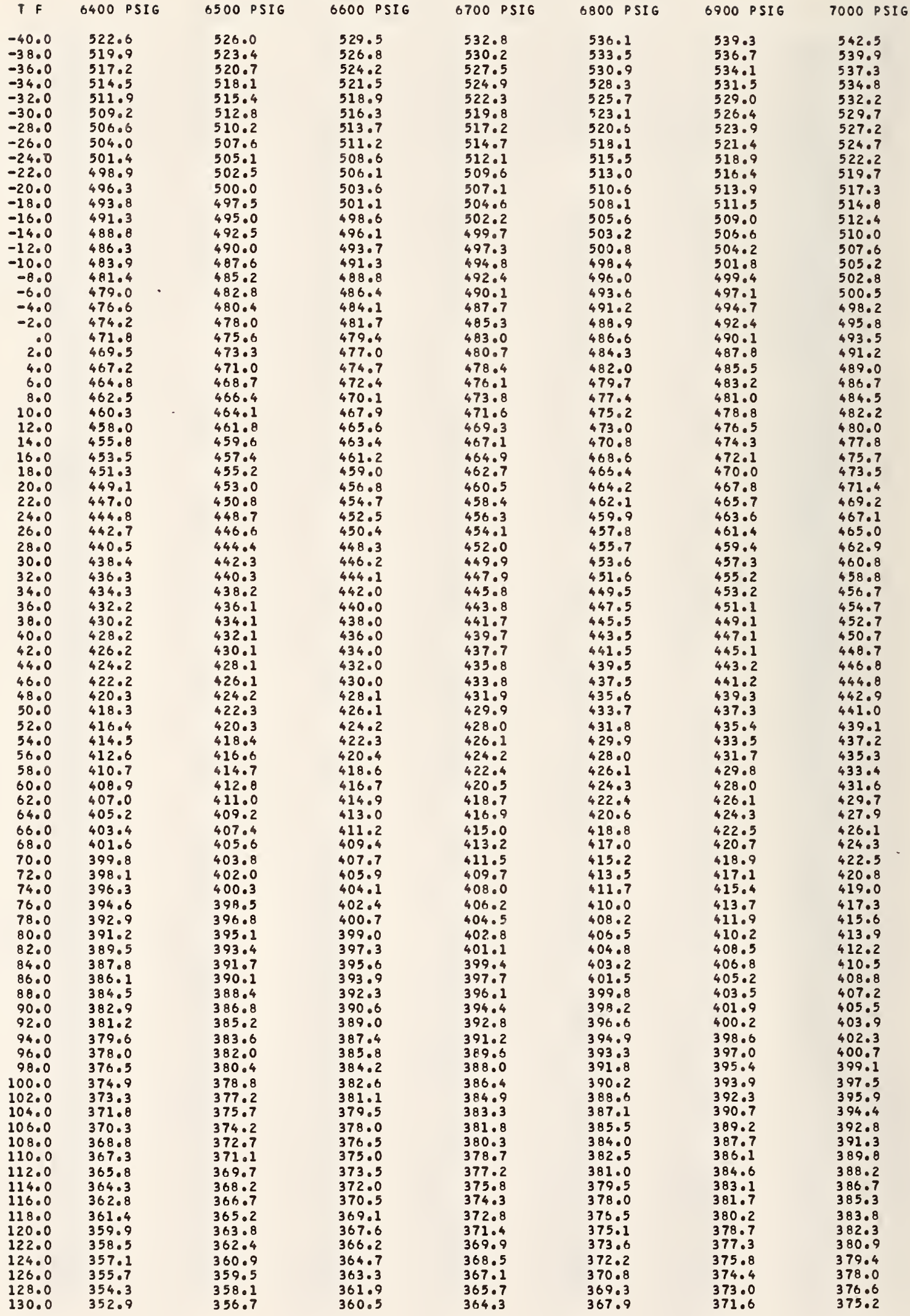


OXYGEN CONTENTS TABLE (SCFICU FT).

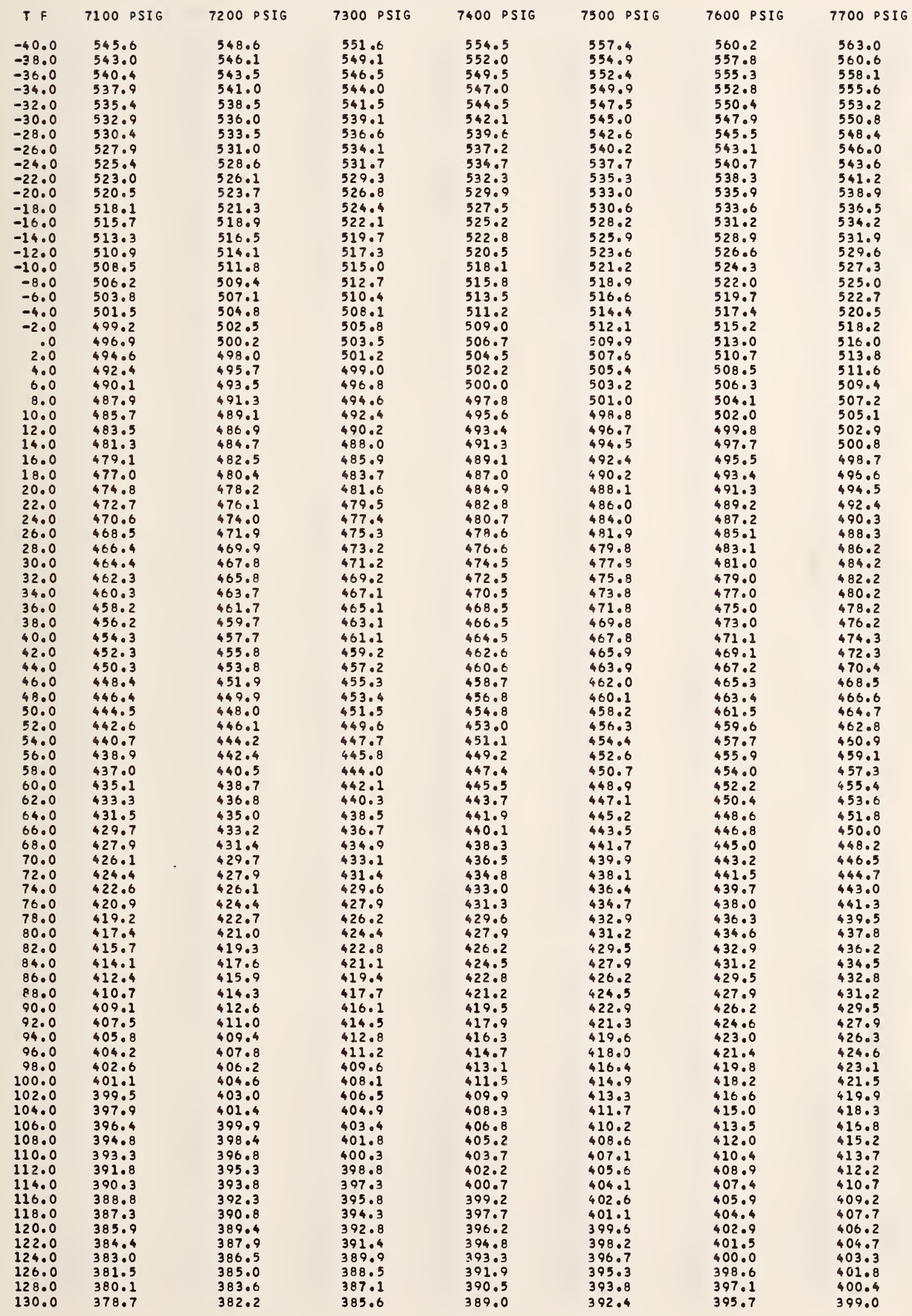


OXYGEN CONTENTS TABLE (SCF/CU FT).

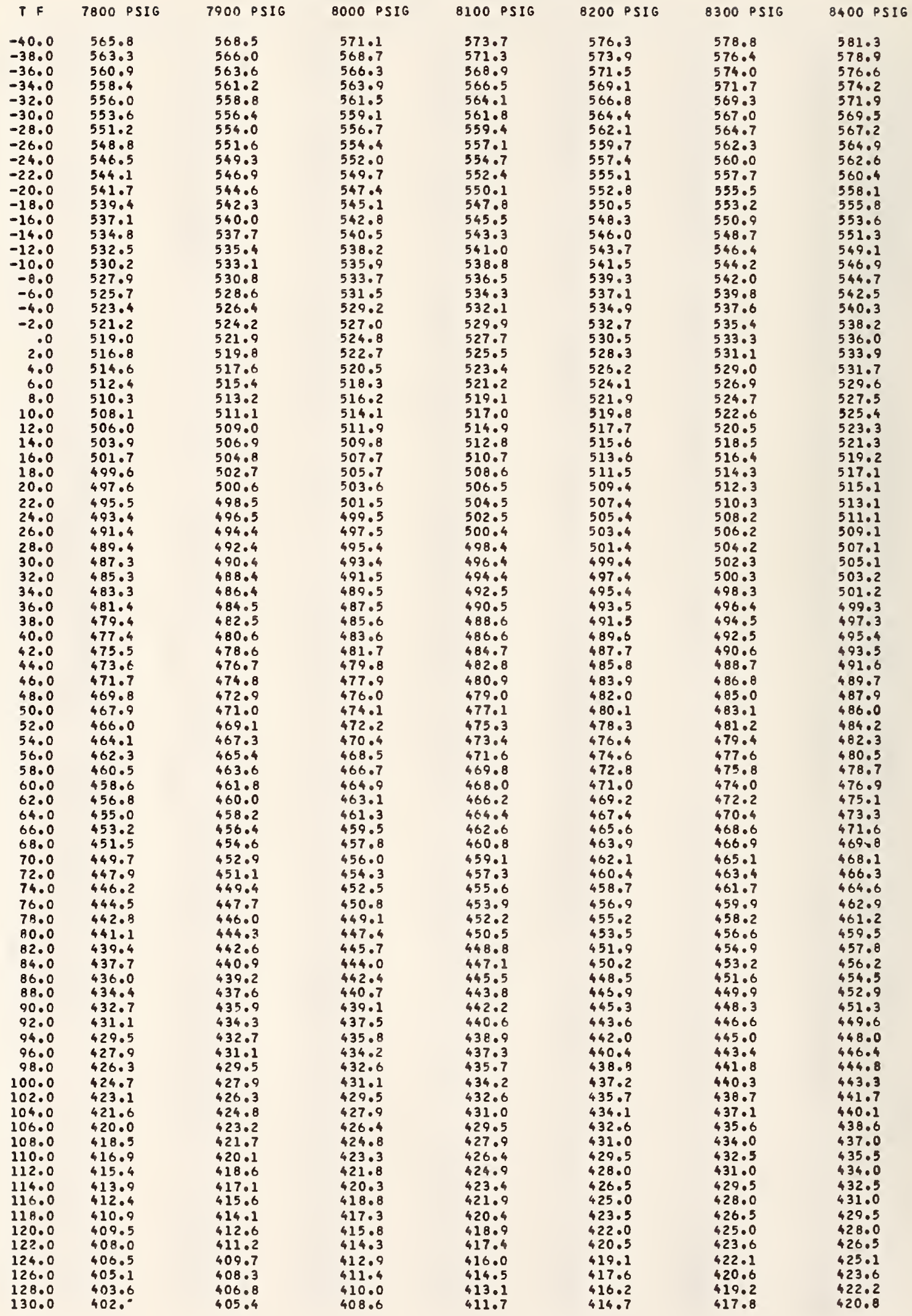


OXYGEN CDNTENTS TABLE (SCF/CU FT).

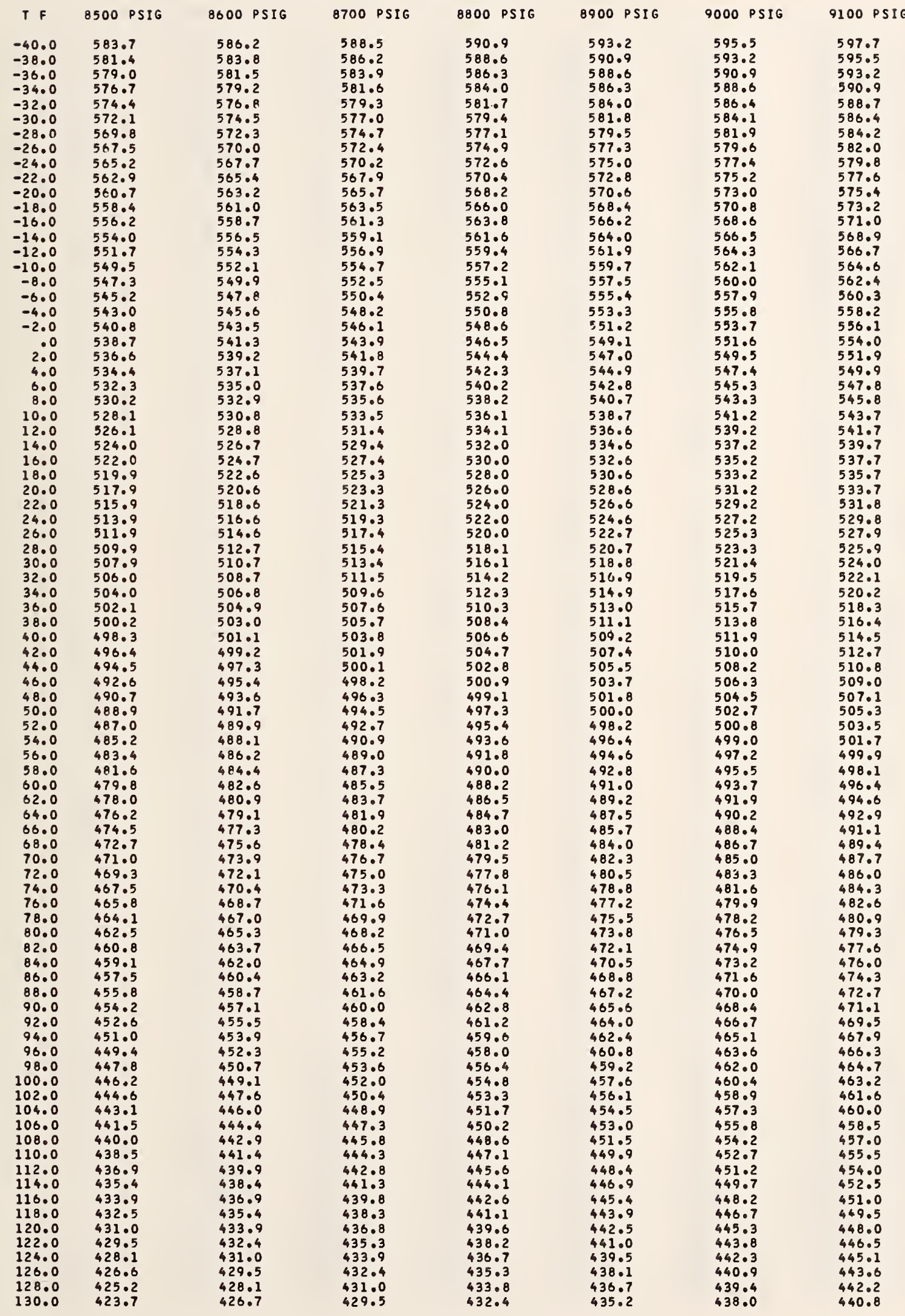


DXYGEN CONTENTS TABLE (SCF/CU FT).

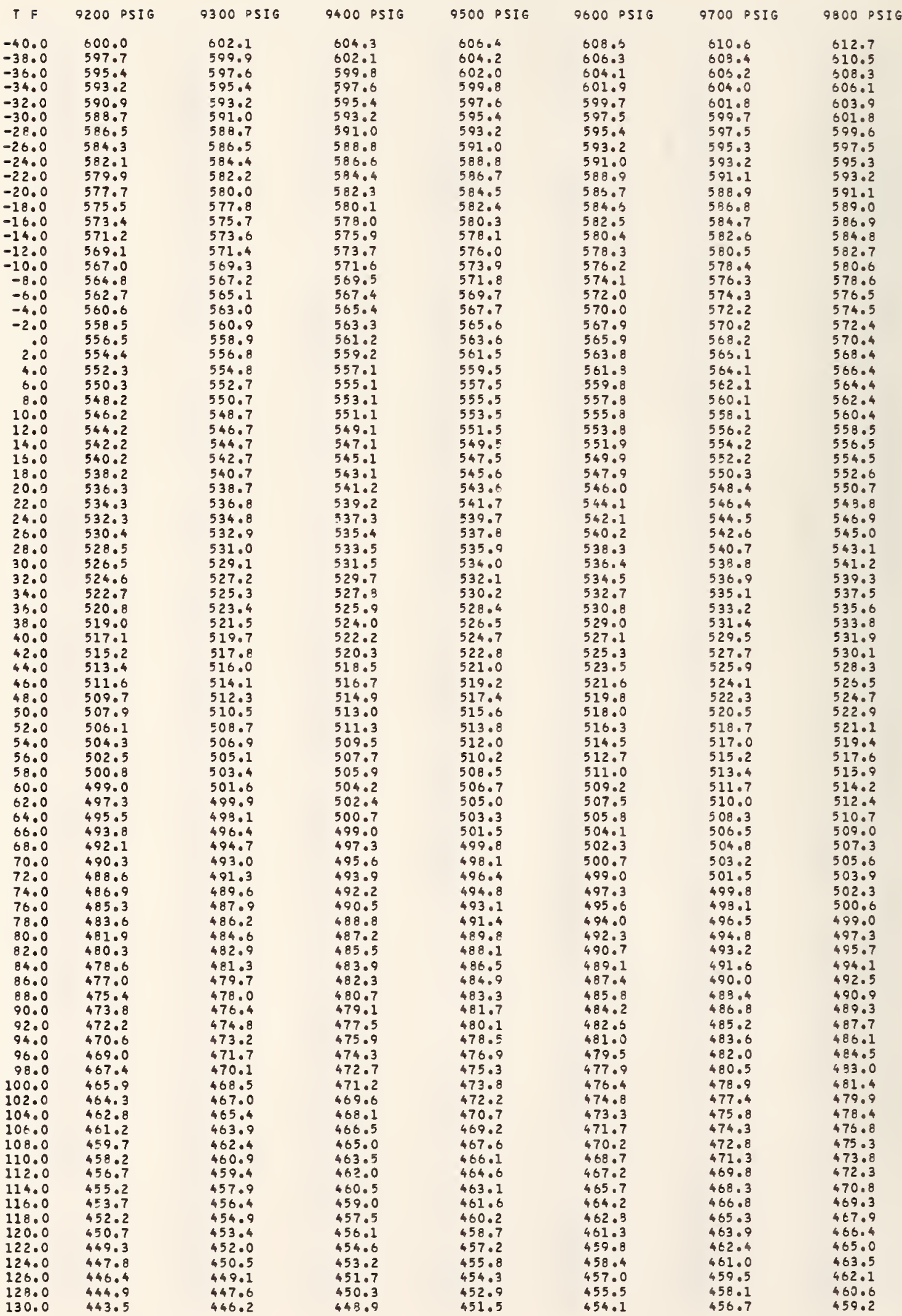


OXYGEN CONTENTS TABLE (SCF/CU FT).

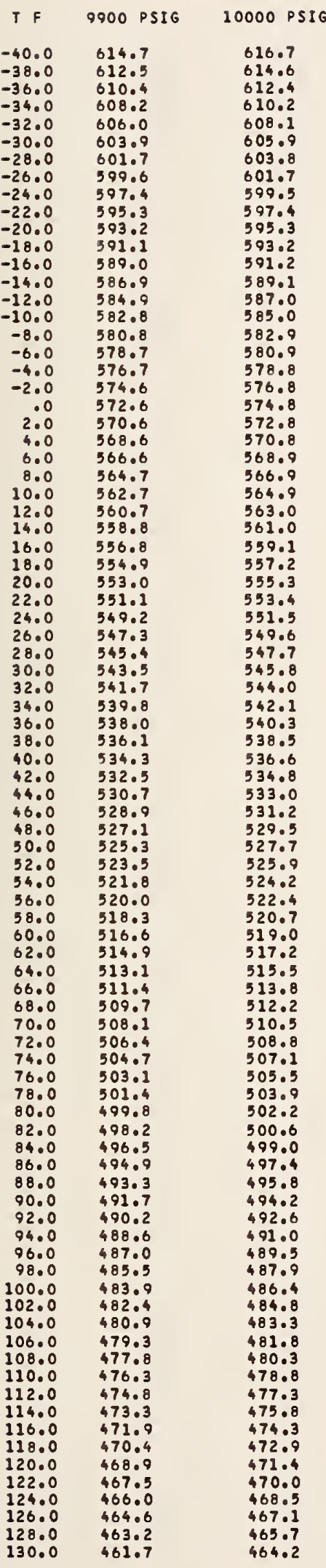


8.2

Argon Contents Table (SCF/cu ft) 
ARGON CONTENTS TABLE (SCF/CU FT).

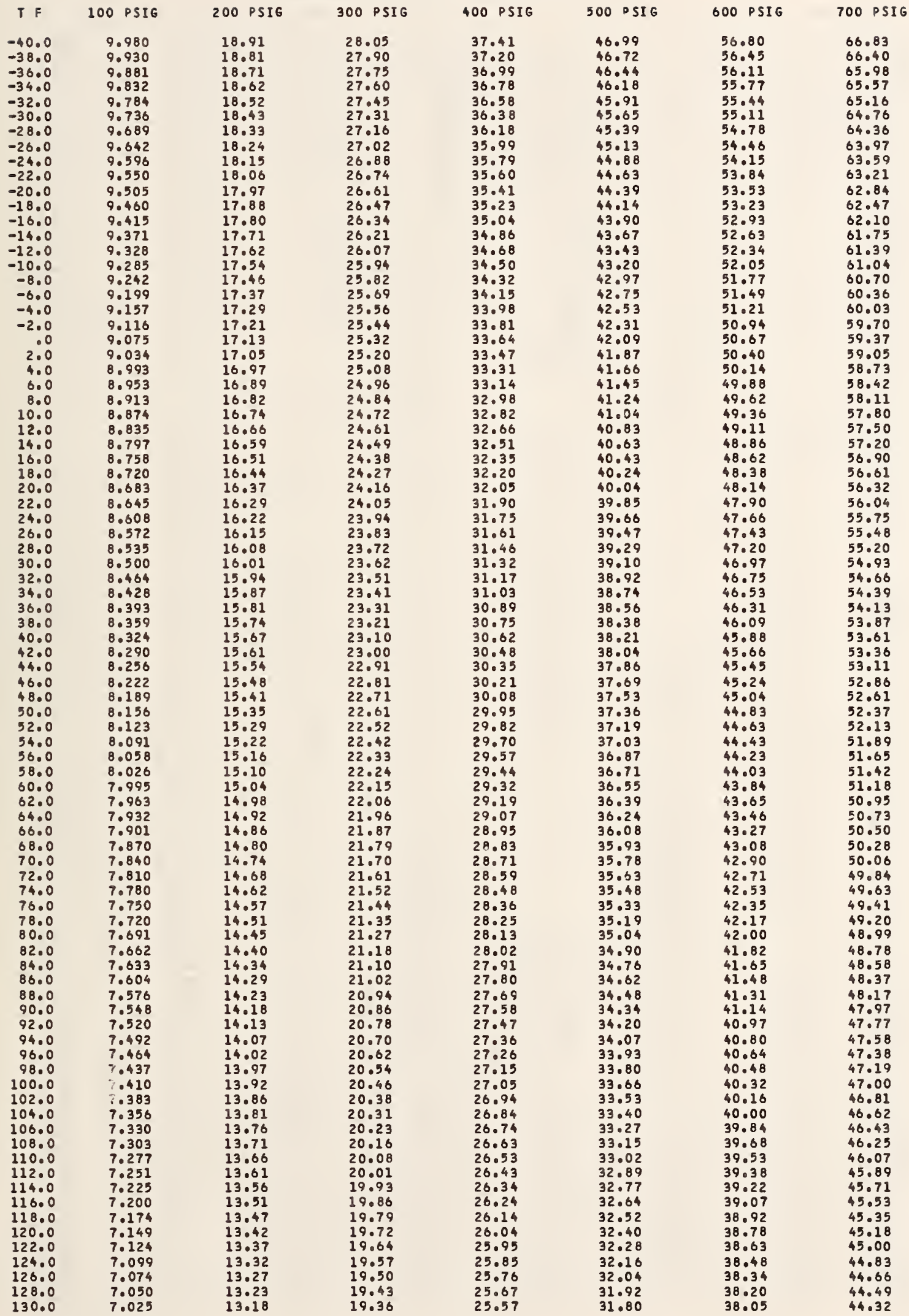


ARGON CONTENTS TABLE (SCFICU FT).

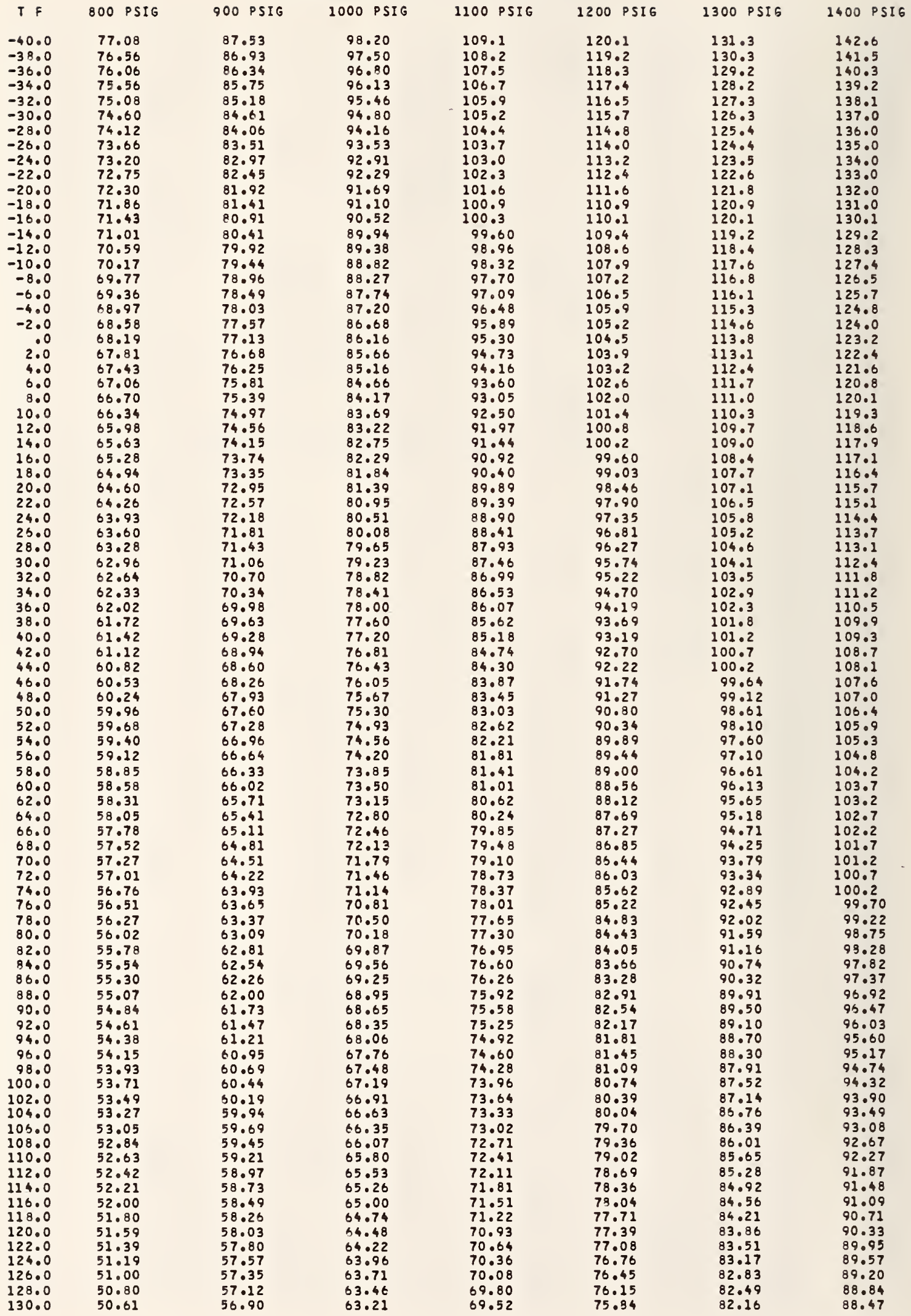


ARGCN CONTENTS TABLE (SCFICU FT).

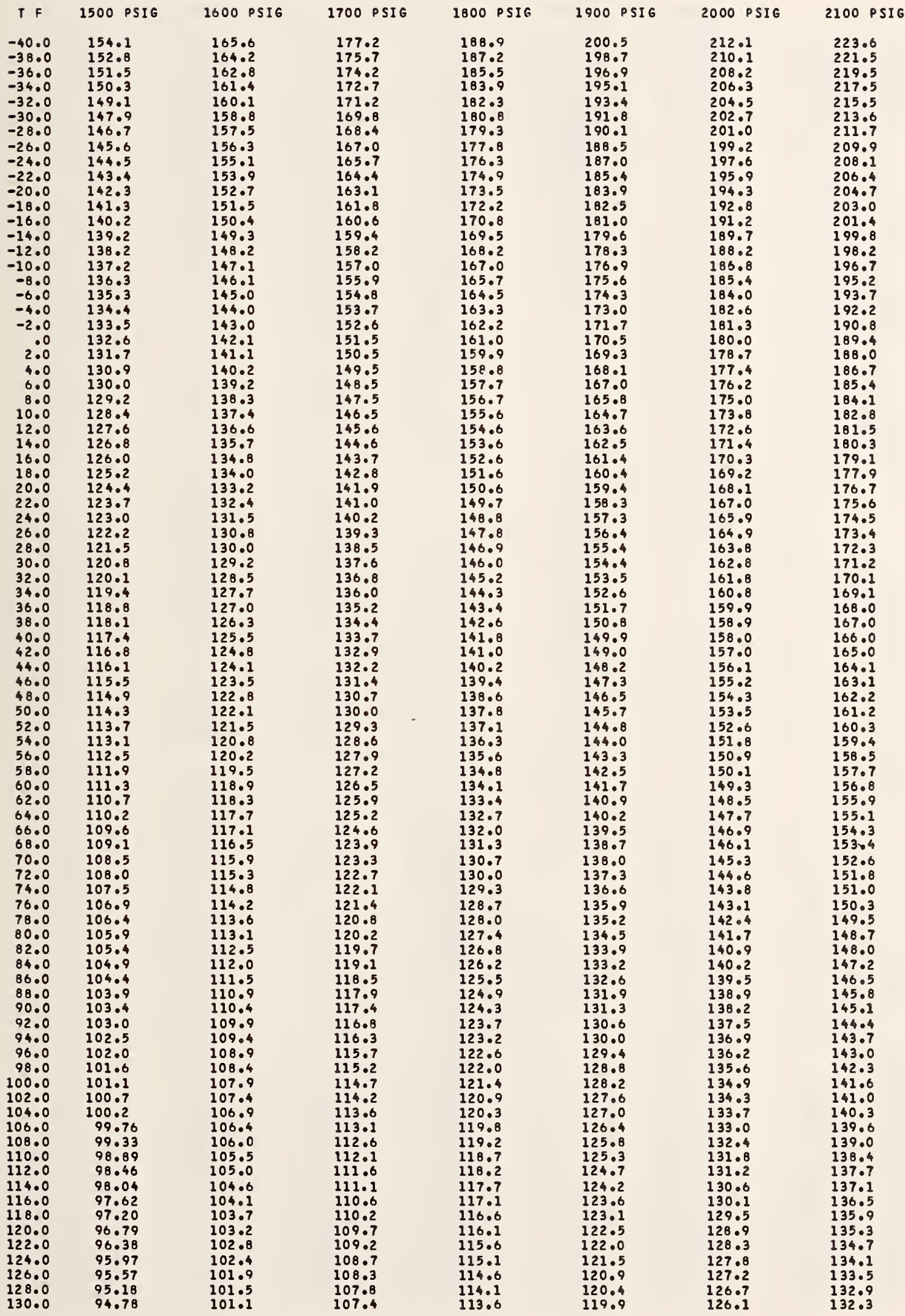


ARGCN CONTENTS TABLE (SCFICU FT).

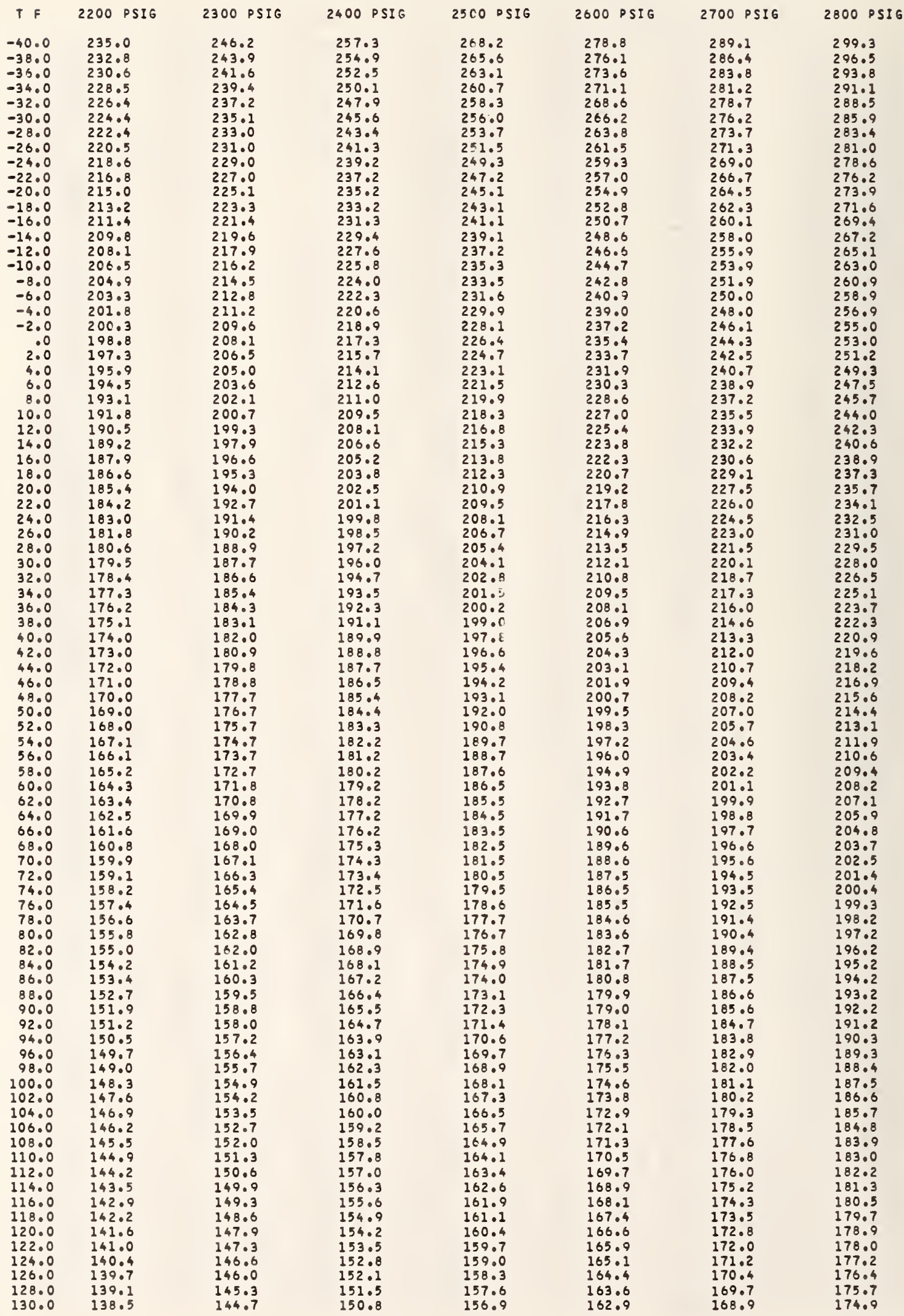


ARGON CONTENTS TABLE (SCF/CU FT).

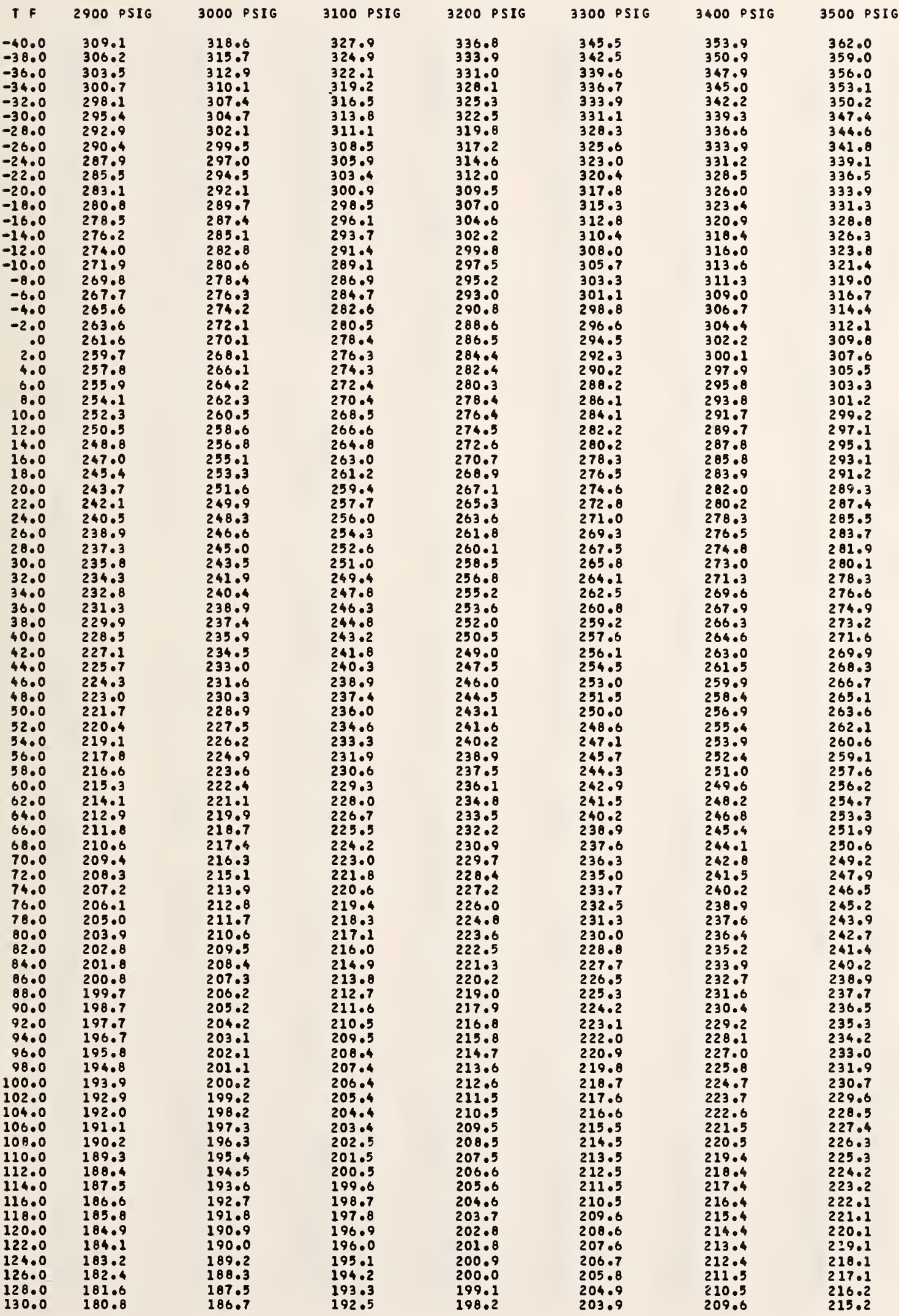




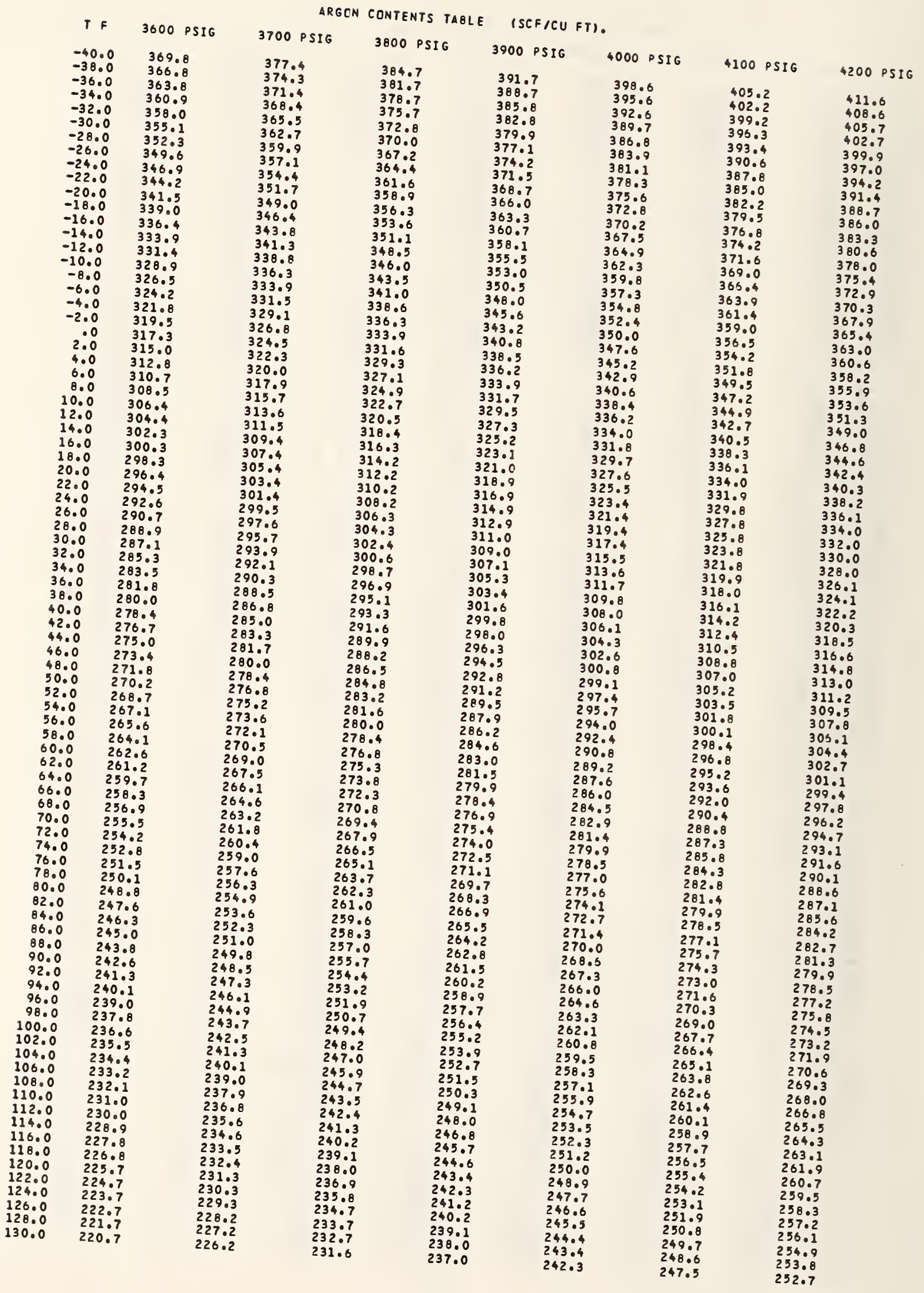


ARGON CONTENTS TABLE (SCFICU FT).

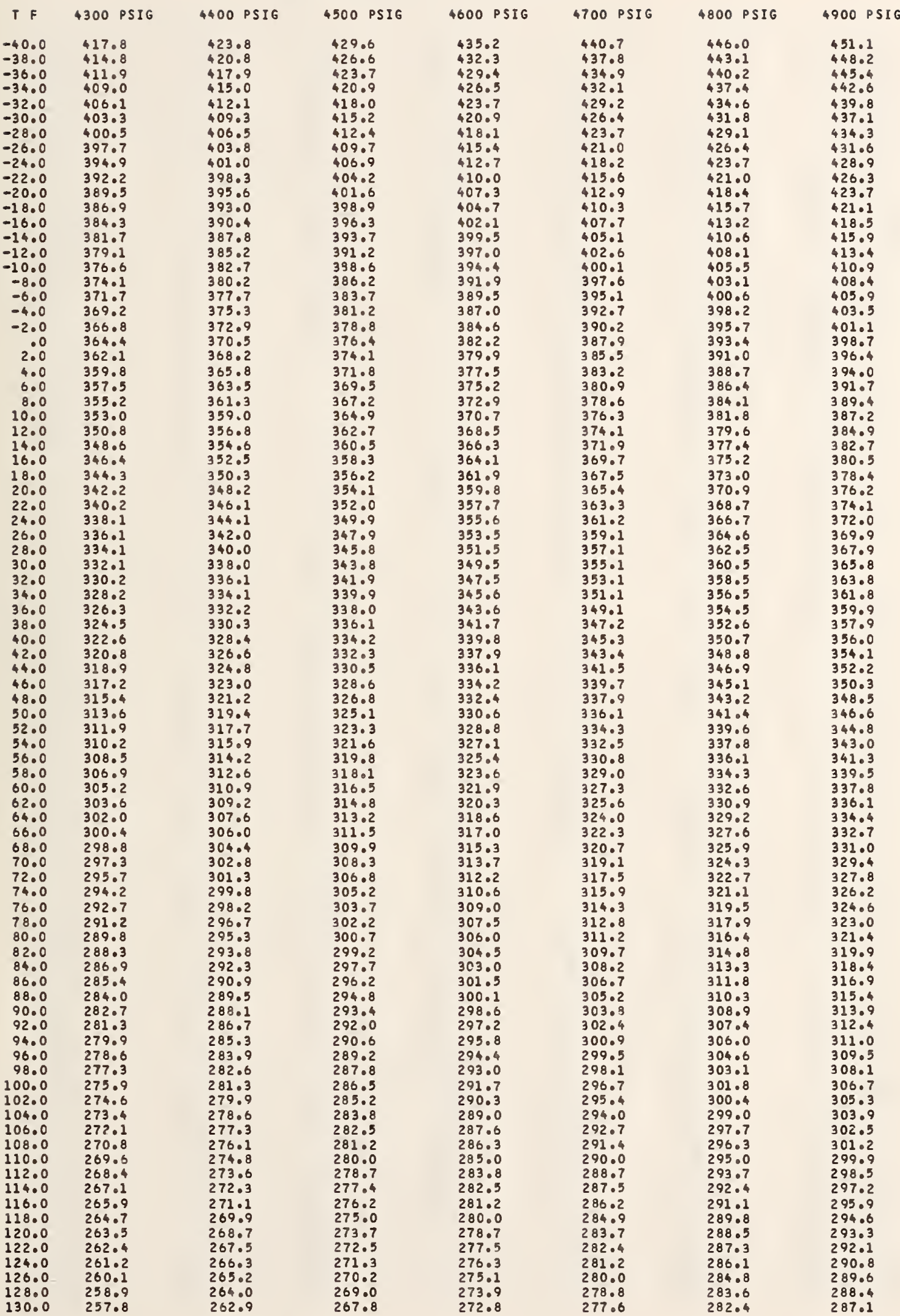




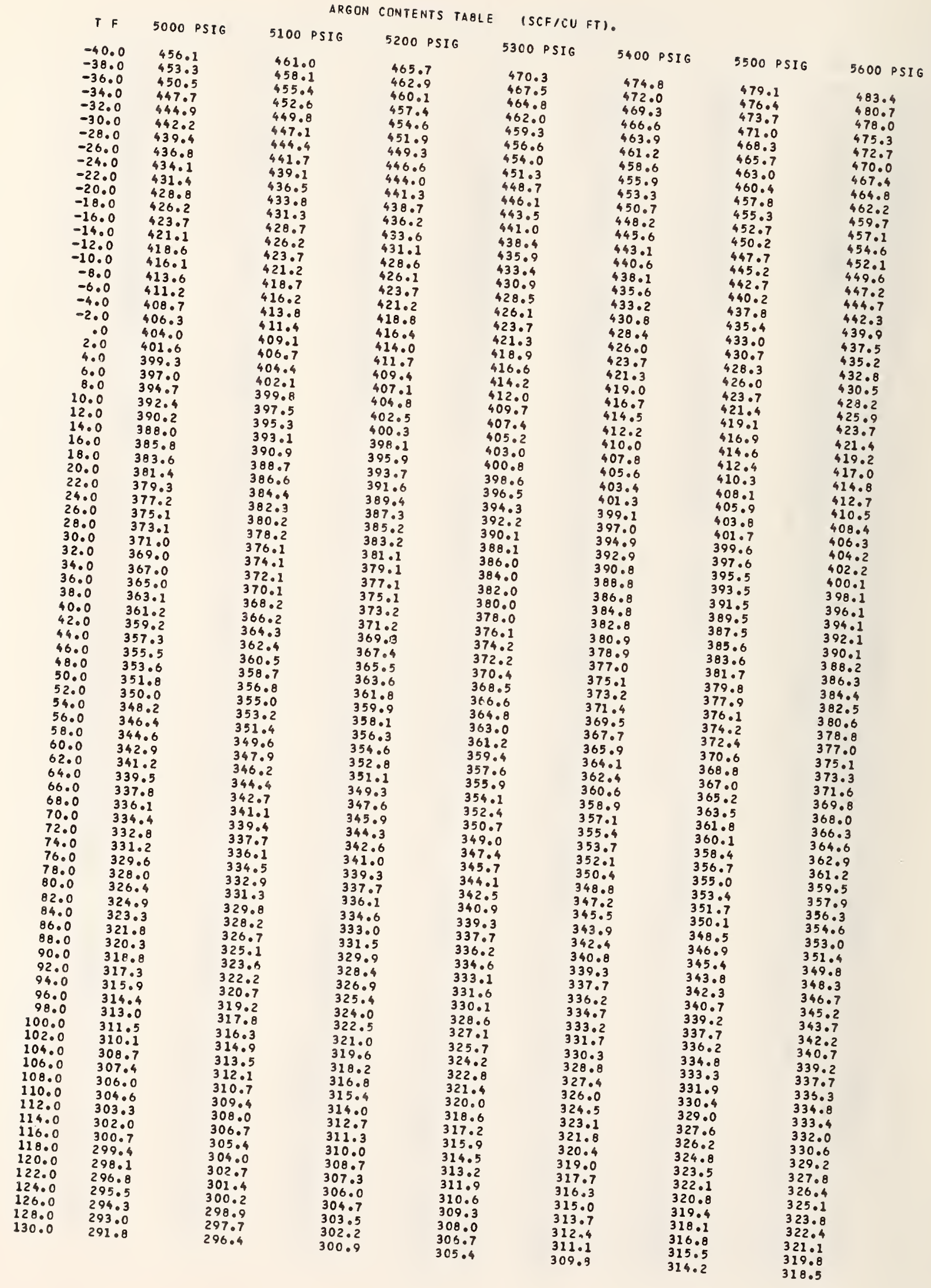


ARGON CONTENTS TABLE (SCF/CU FT).

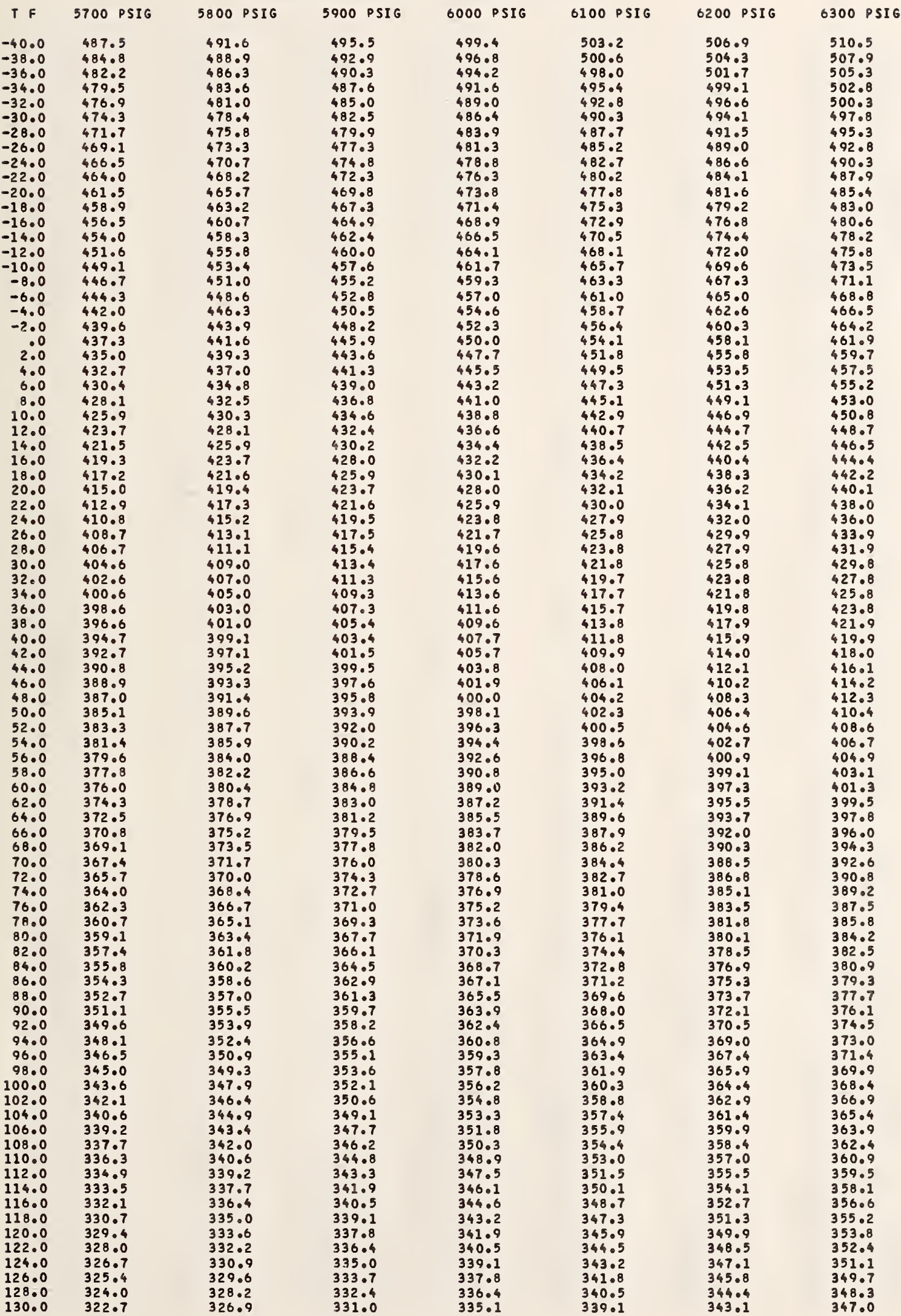


ARGCN CONTENTS TABLE (SCF/CU FT).

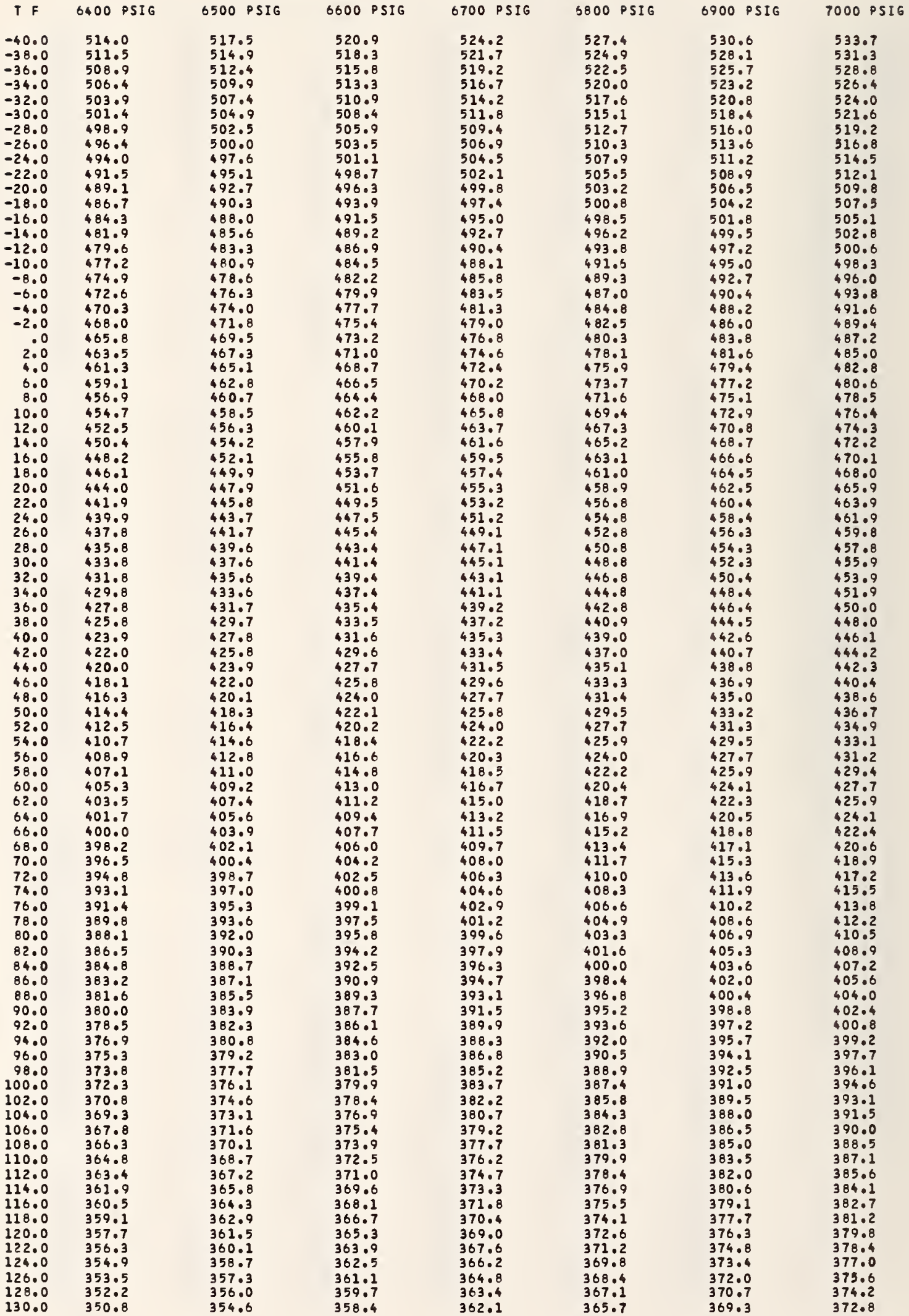


ARGON CONTENTS TABLE (SCFICU FT).

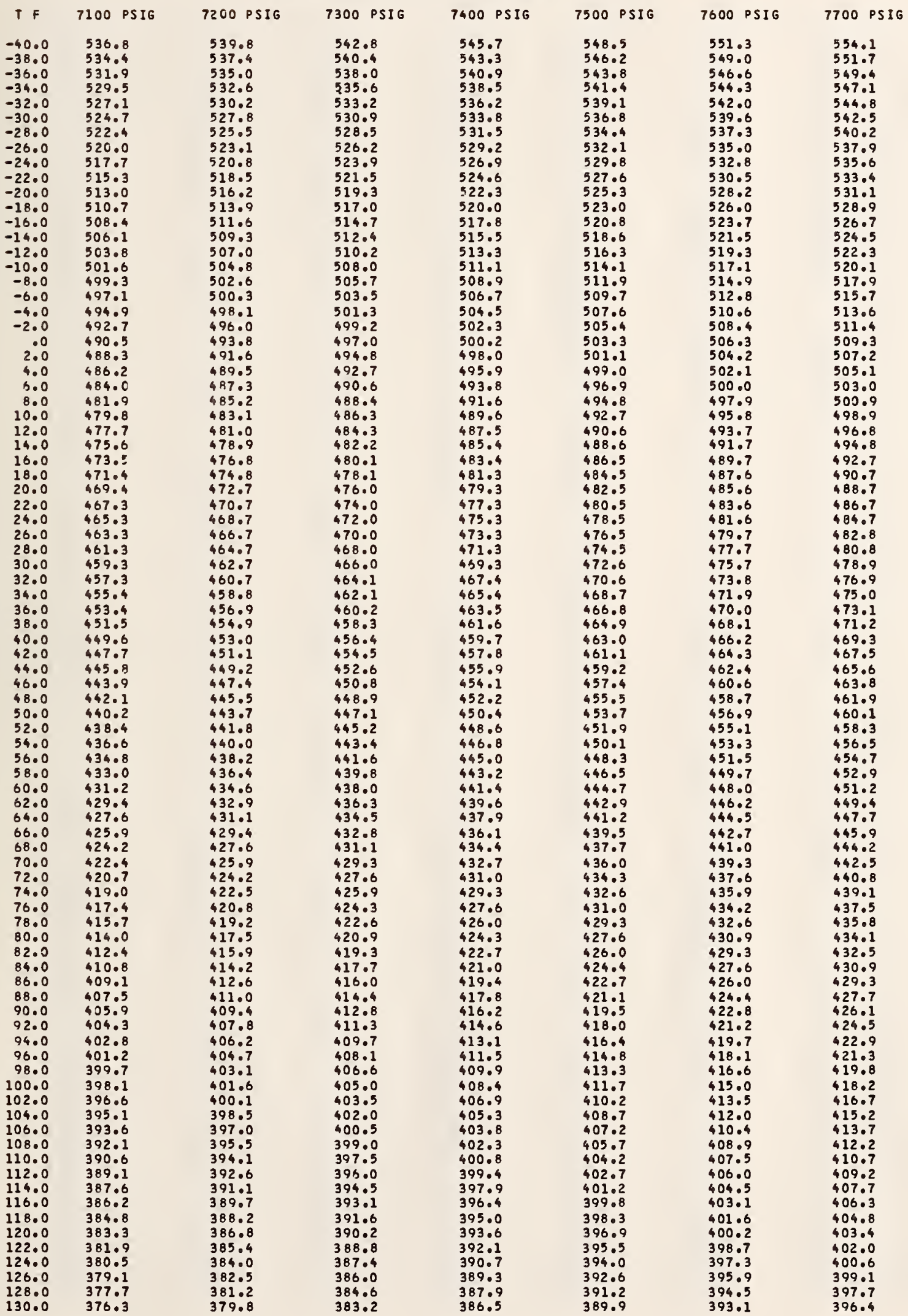


ARGON COYTENTS TABLE (SCFICU FT).

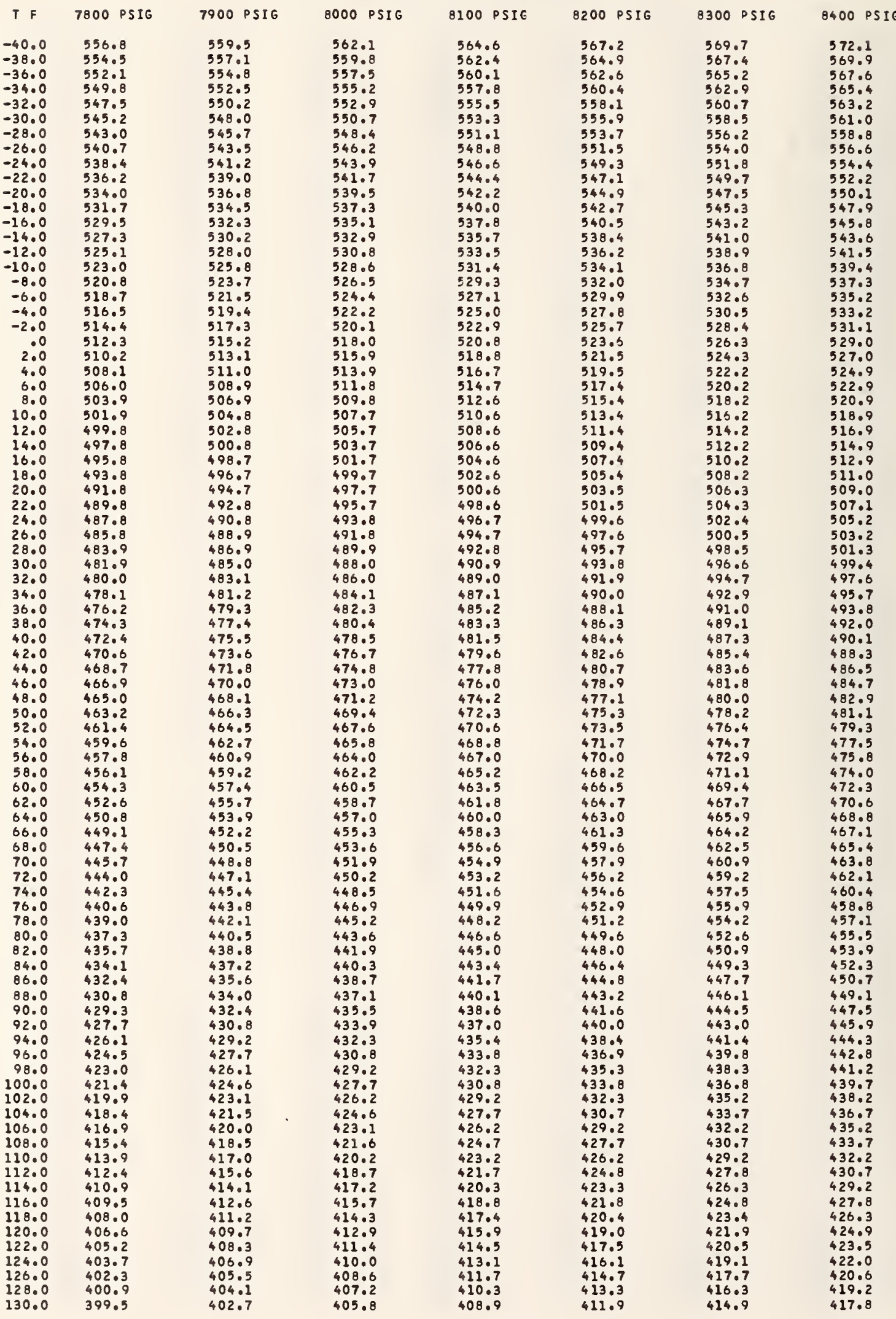


ARGON CONTENTS TABLE (SCFICU FT).

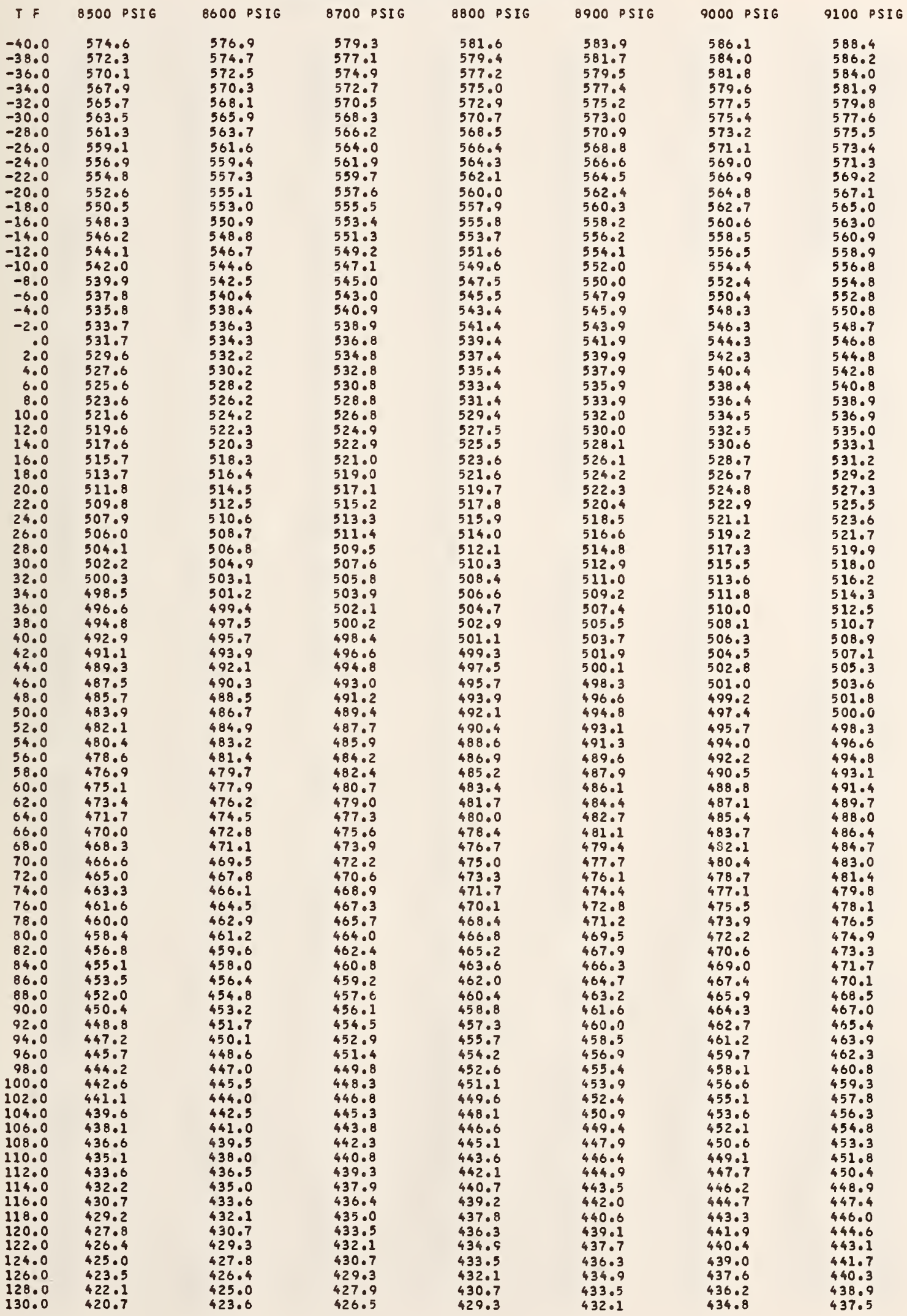


ARGON CONTENTS TABLE (SCF/CU FT).

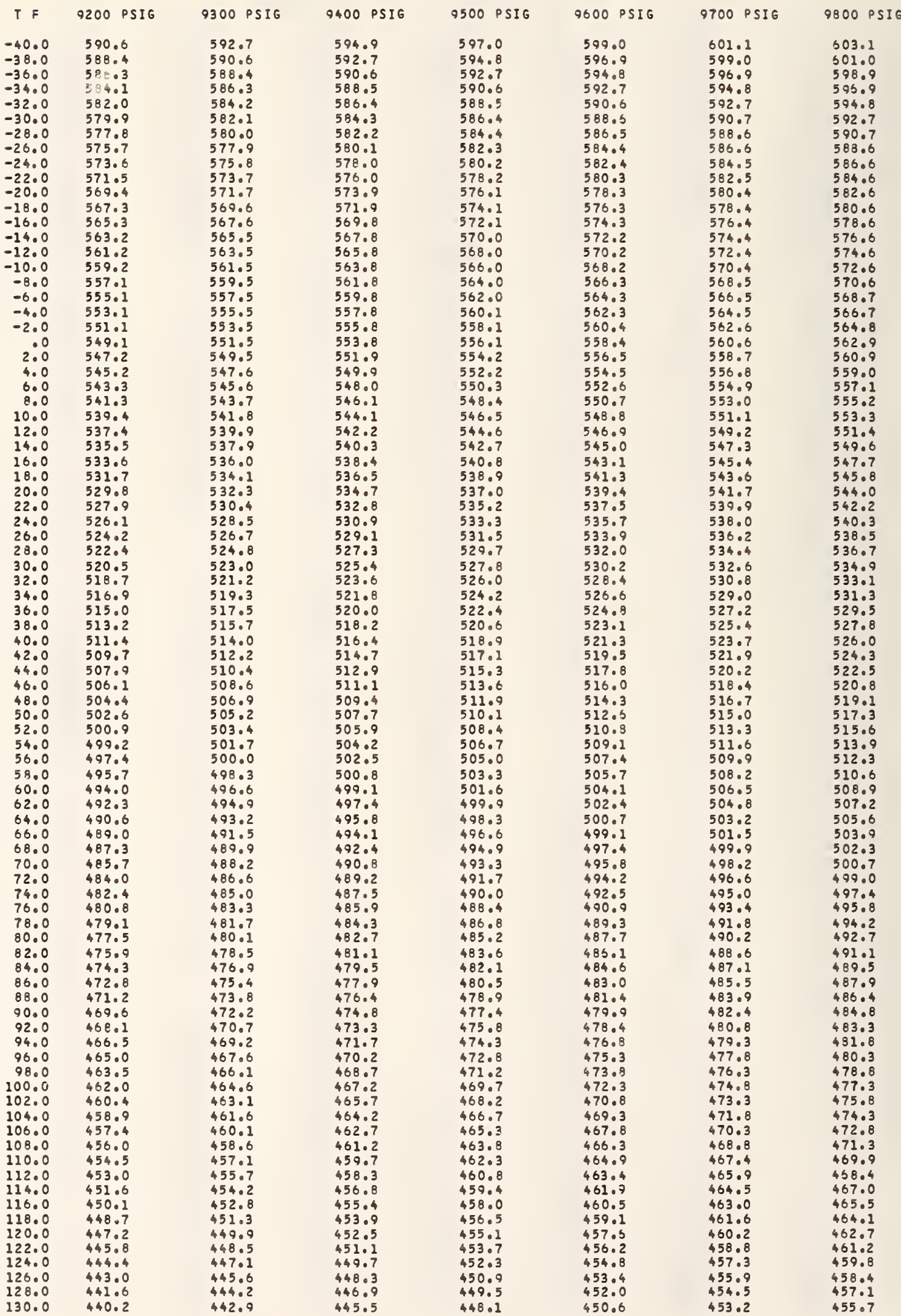


ARGON CONTENTS TABLE (SCF/CU FT).

TF 9900 PSIG 10000 PSIG

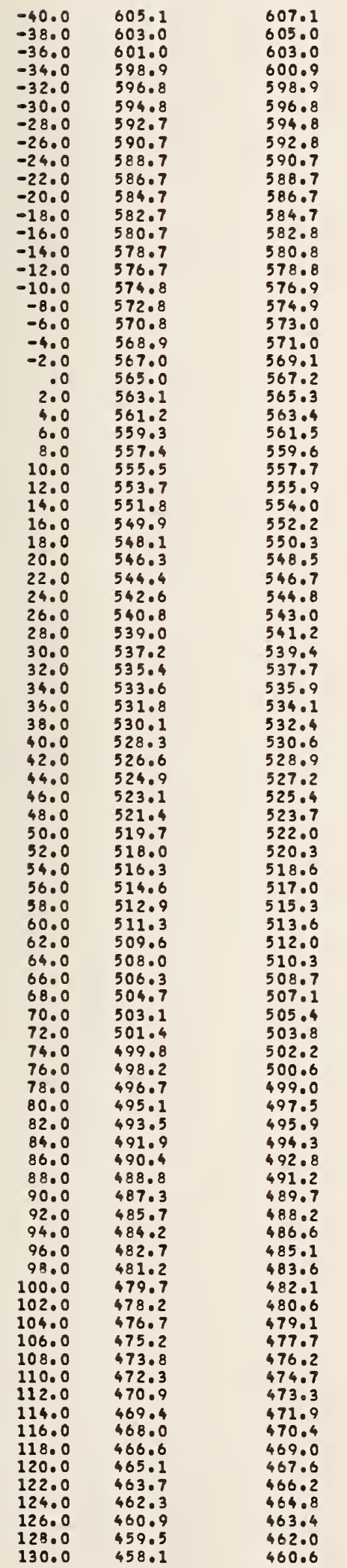


8.3

Nitrogen Contents Table (SCF/cu ft) 
NITROGEN CONTENTS TABLE (SCF/CU FT).

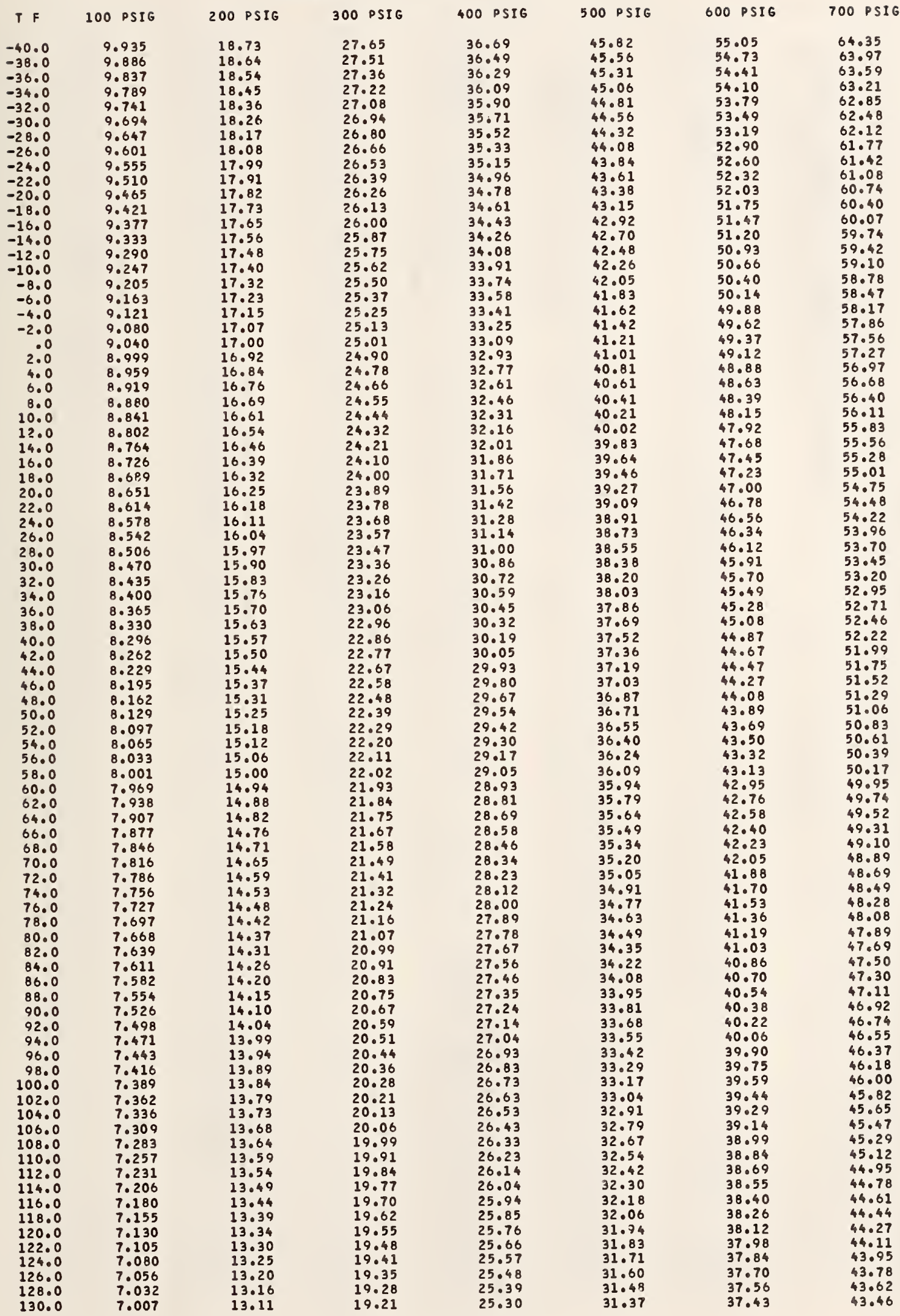


NITROGEN CONTENTS TABLE (SCFICU FT).

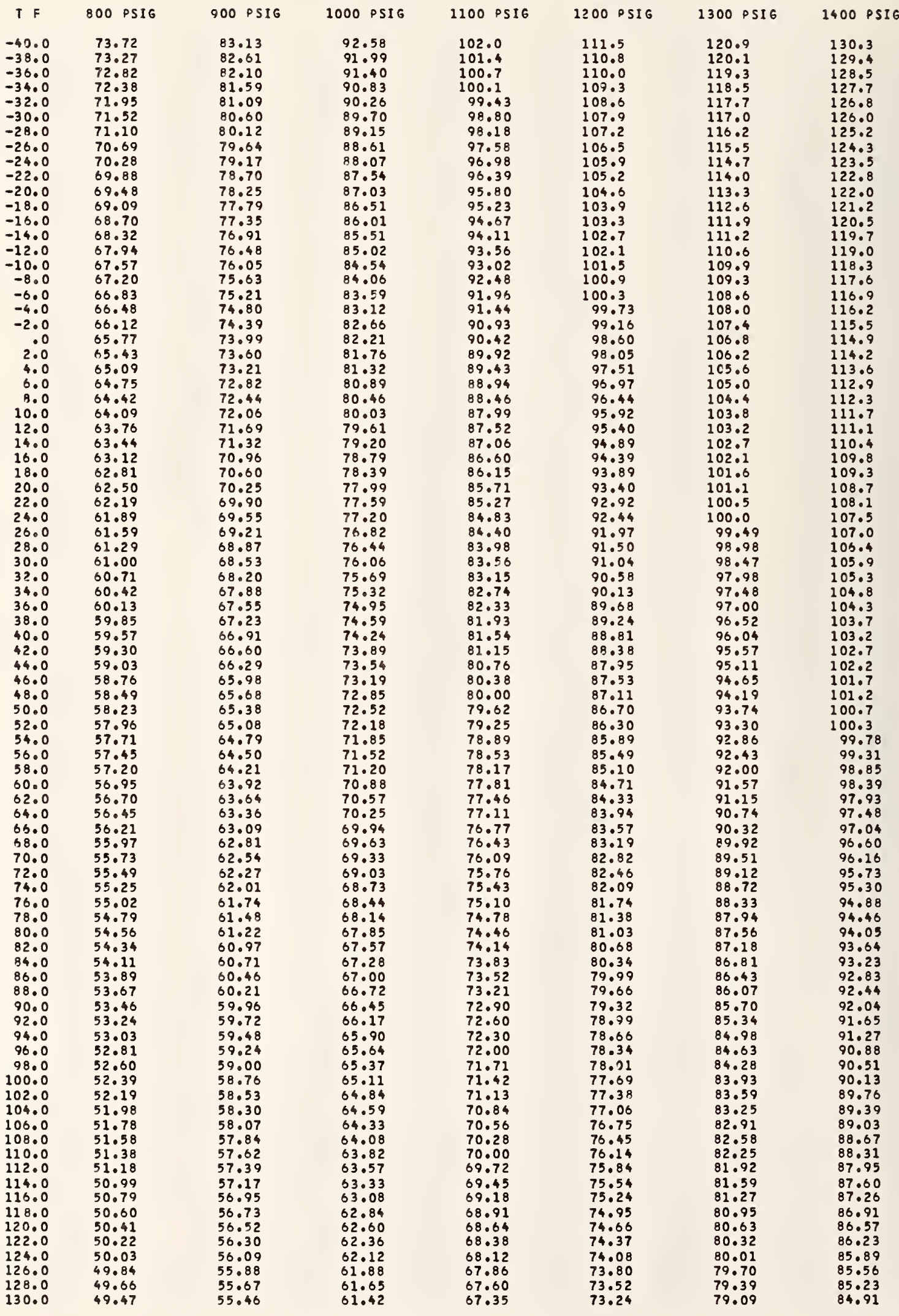


NITRDGEN CONTENTS TABLE (SCF/CU FT).

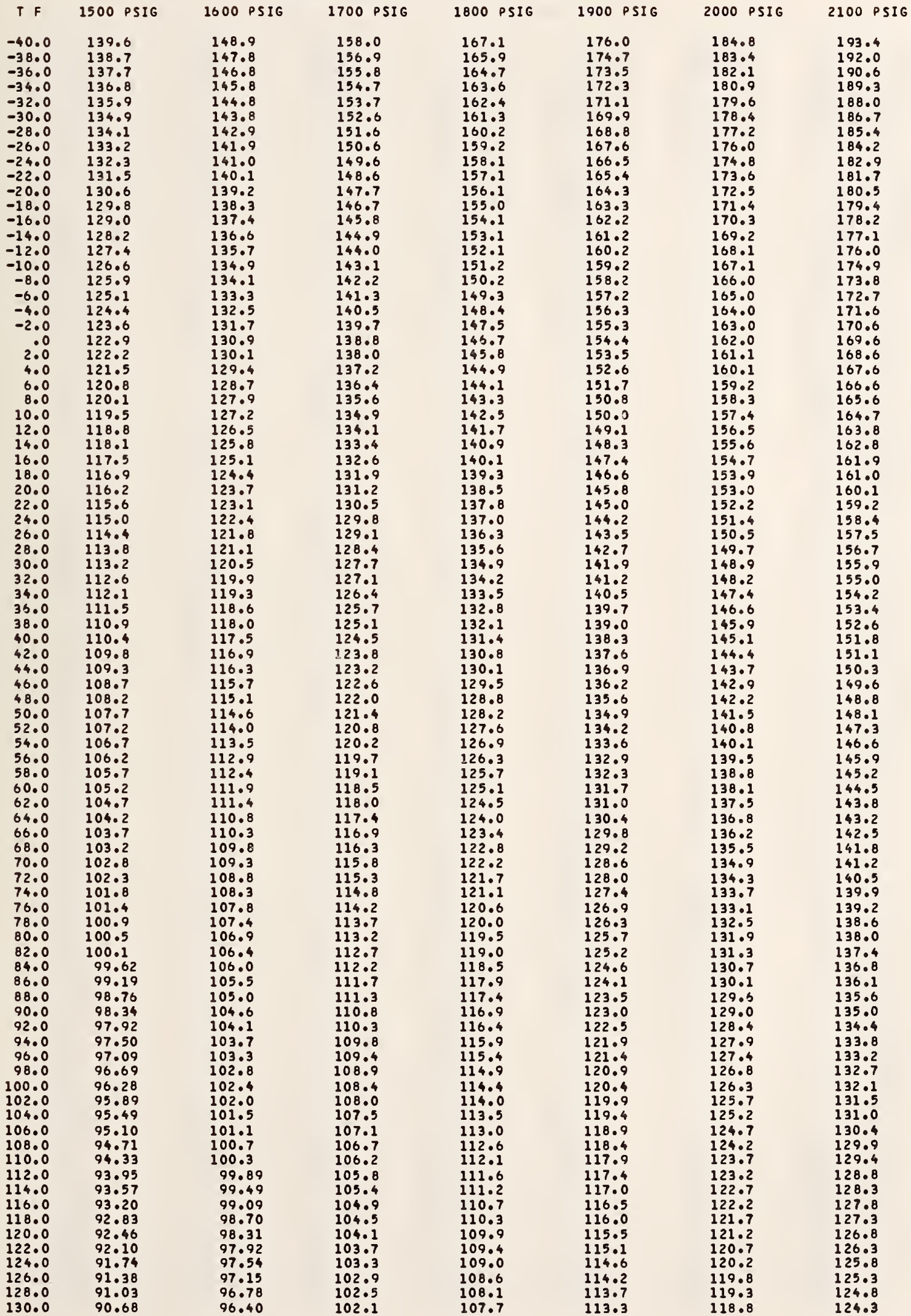


NITROGEN CONTENTS TABLE (SCF/CU FT).

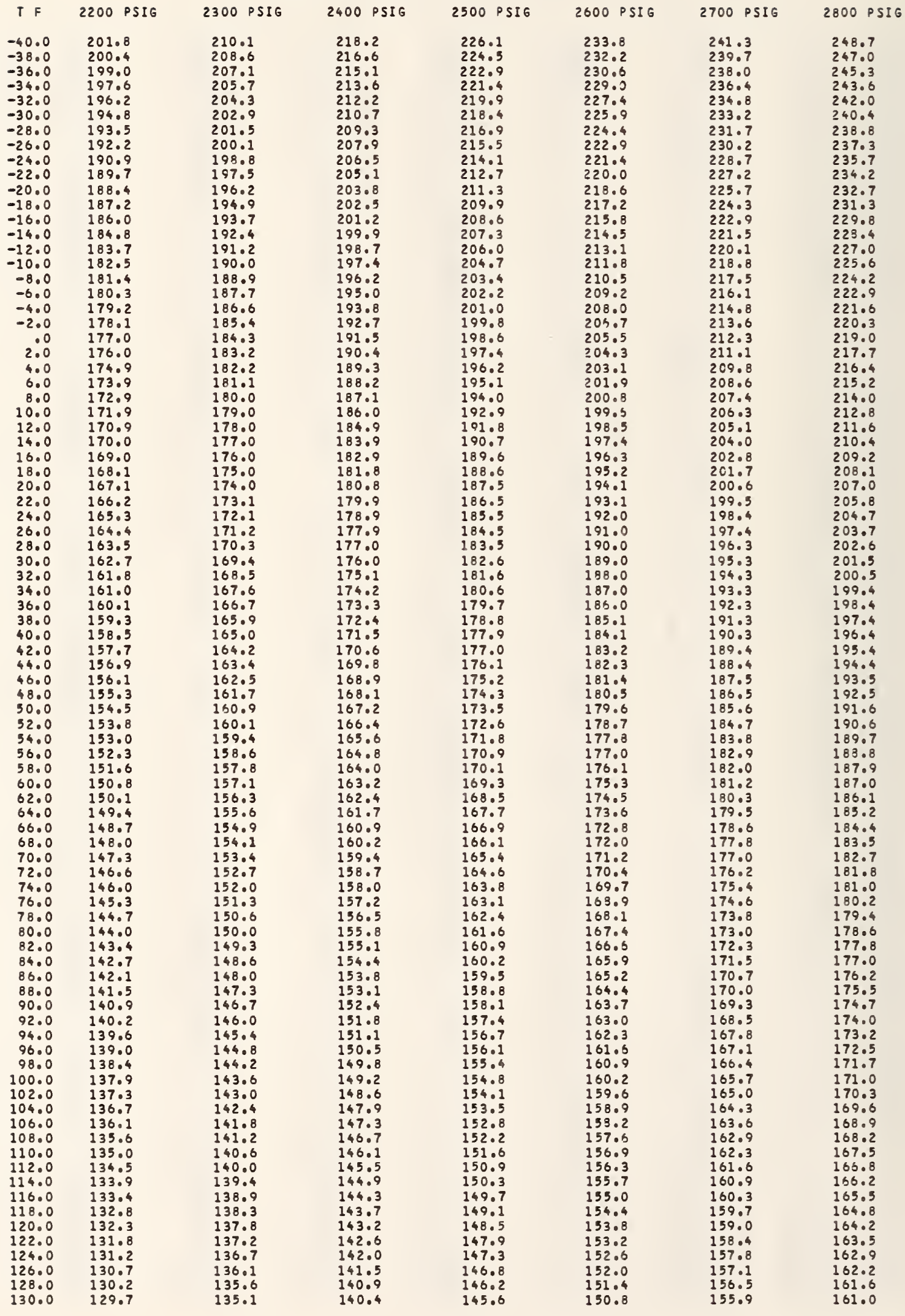


TF 2900 PSIG 3000 PSIG 3100 PSITROGEN CONTENTS TABLE (SCFICU FT)

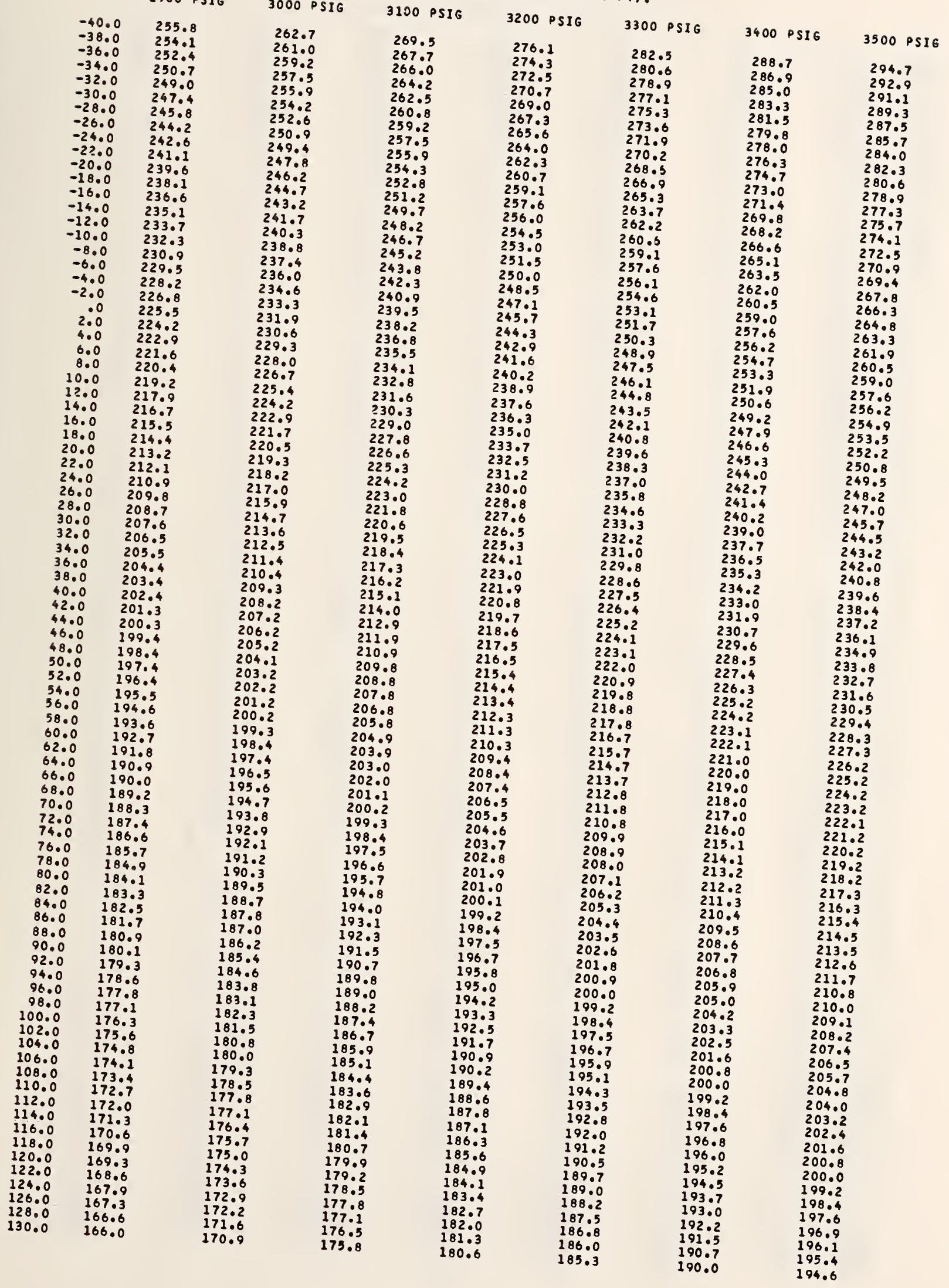


NITROGEN CONTENTS TABLE (SCF/CU FT).

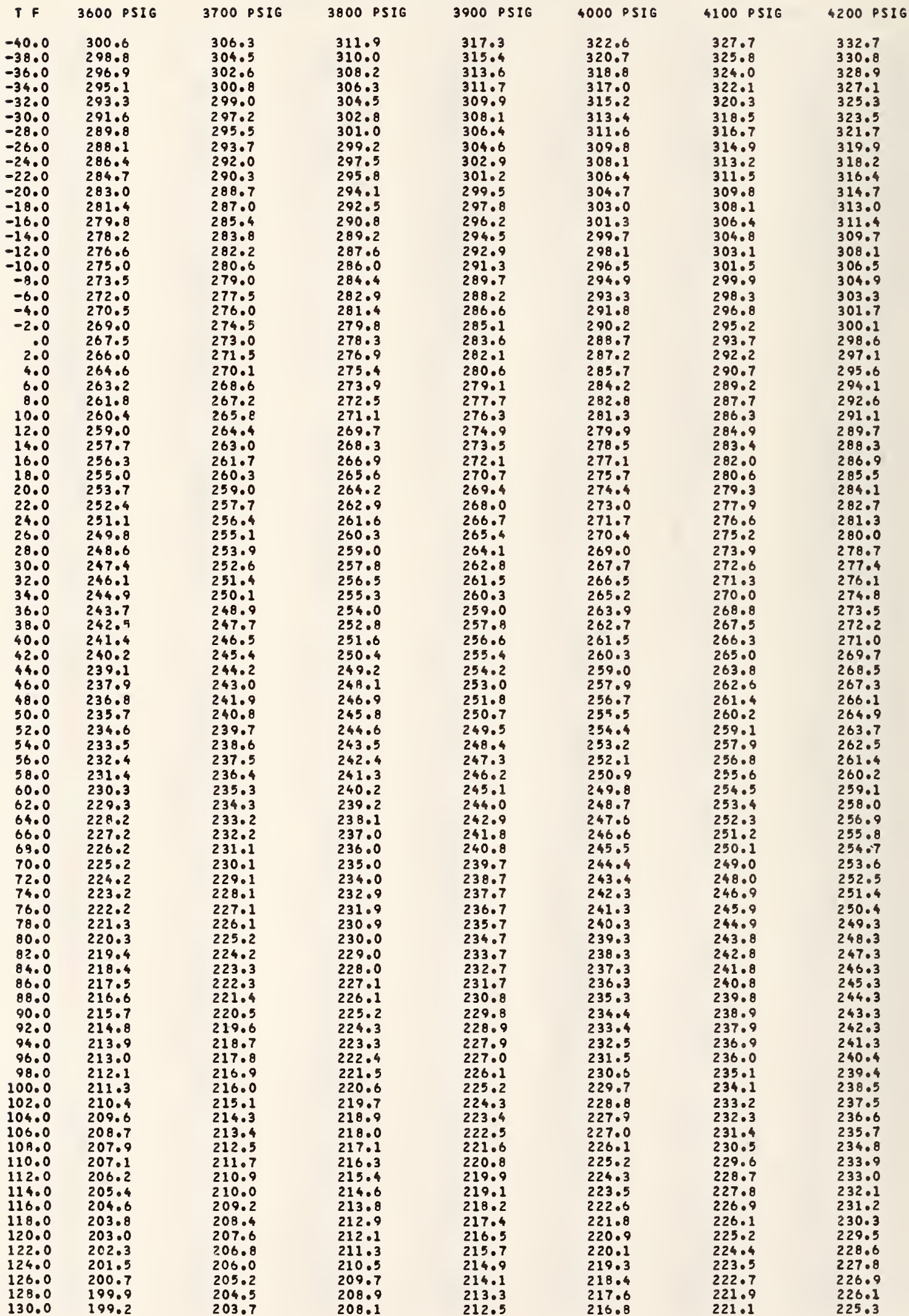


NITROGEN CONTENTS TABLE (SCF/CU FT).

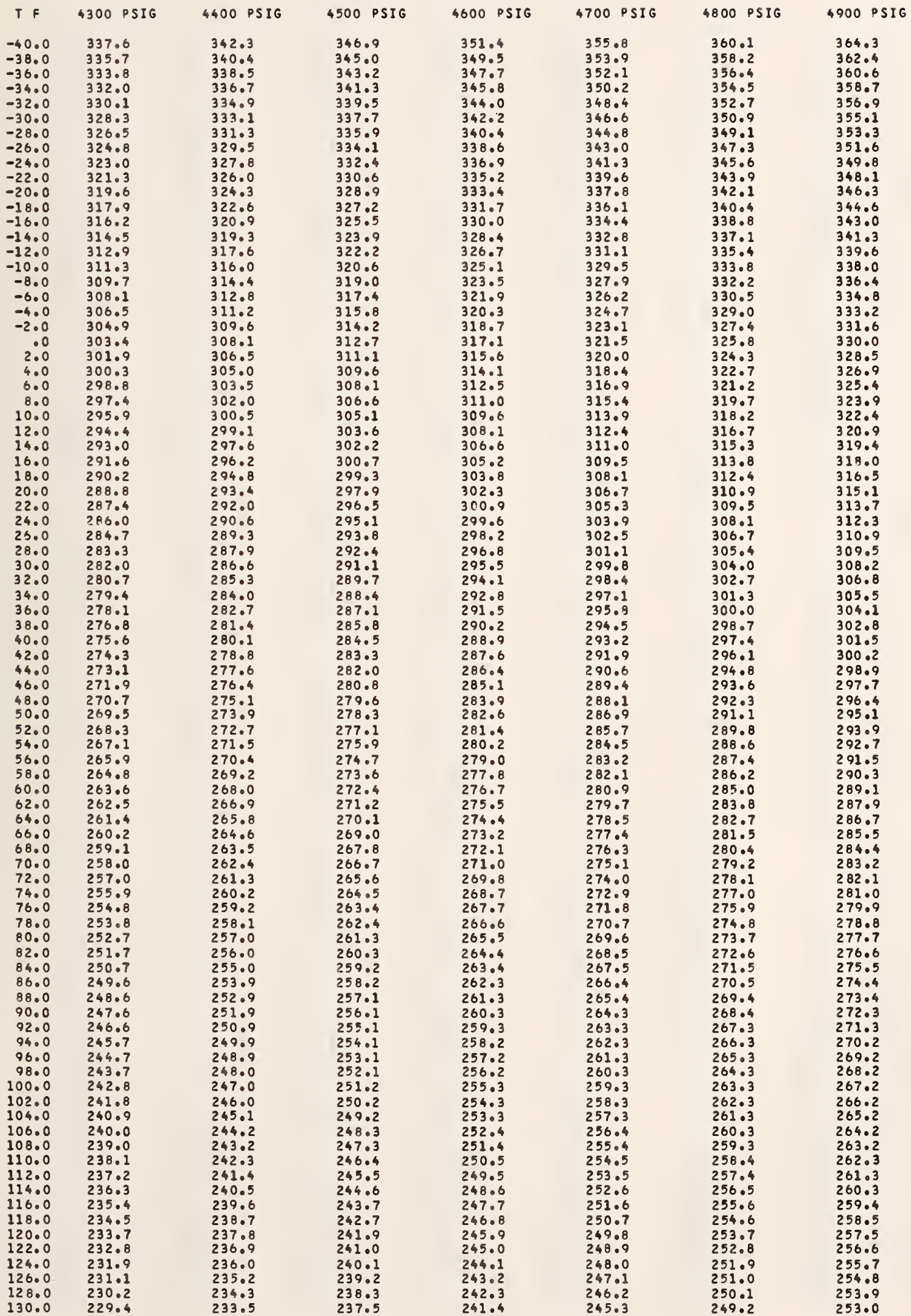


NITROGEN CONTENTS TABLE (SCF/CU FT).

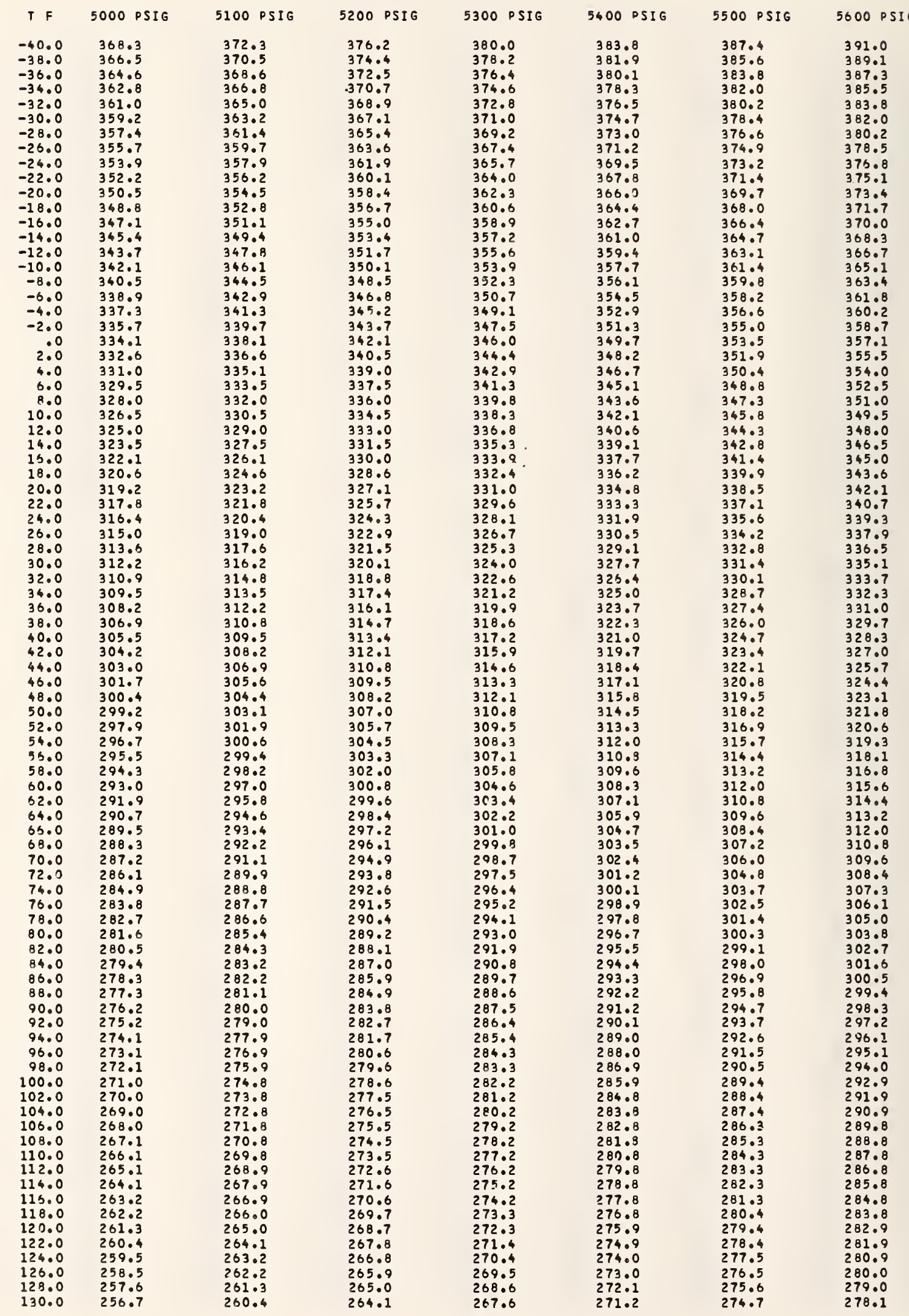


NITROGEN CONTENTS TABLE (SCF/CU FT).

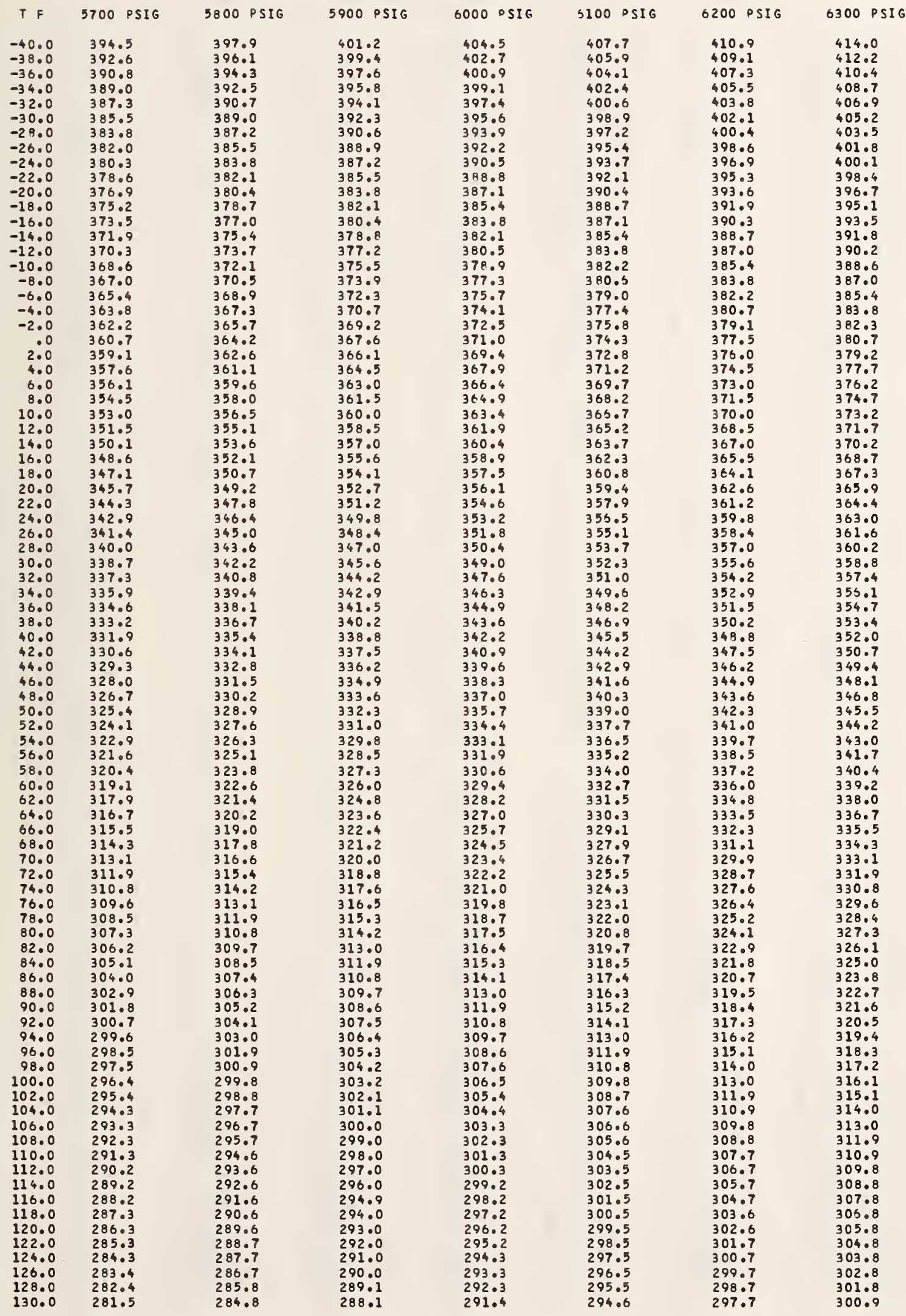


NITROGEN CONTENTS TABLE (SCF/CU FT).

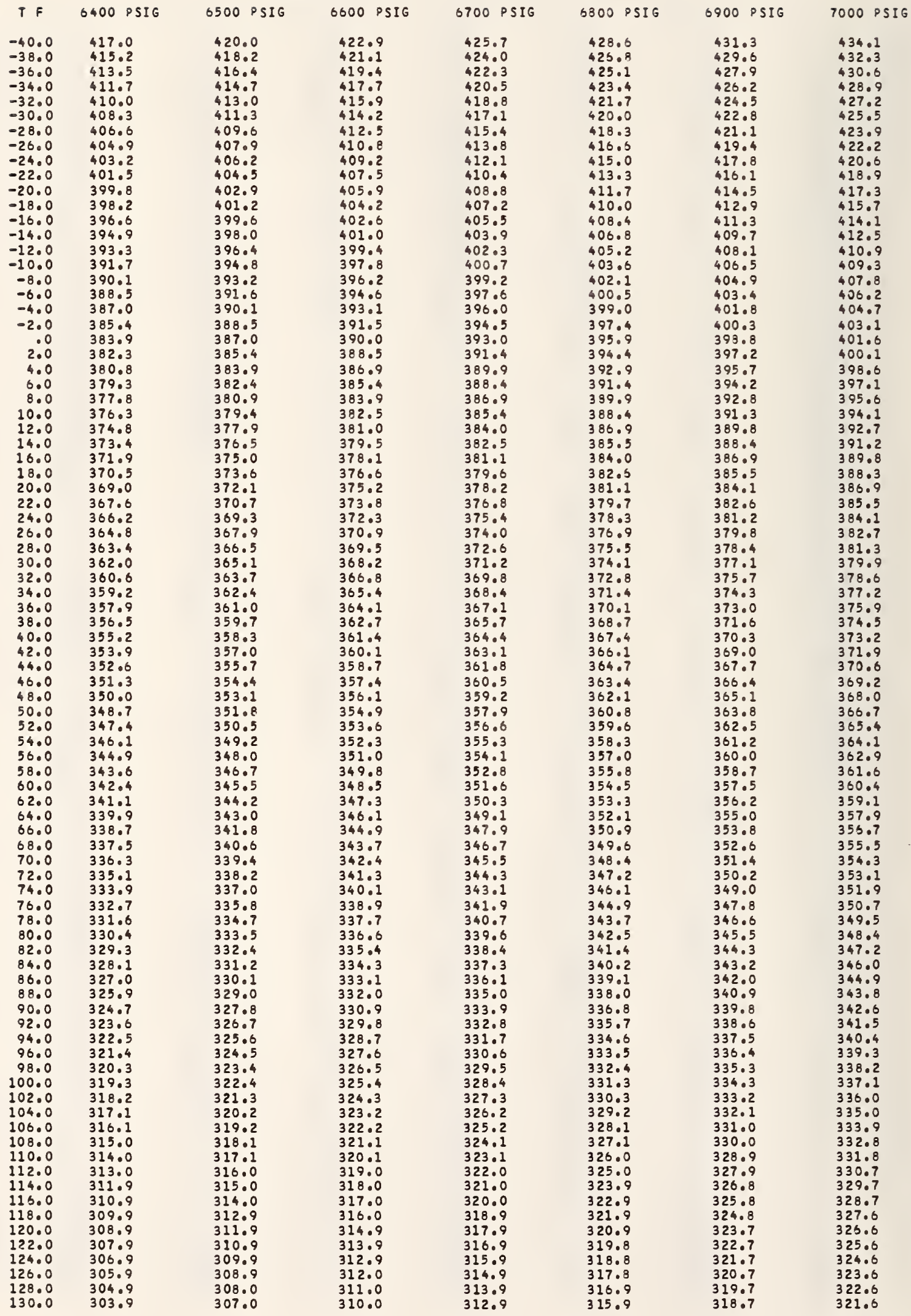


NITROGEN CONTENTS TABLE (SCFICU FT).

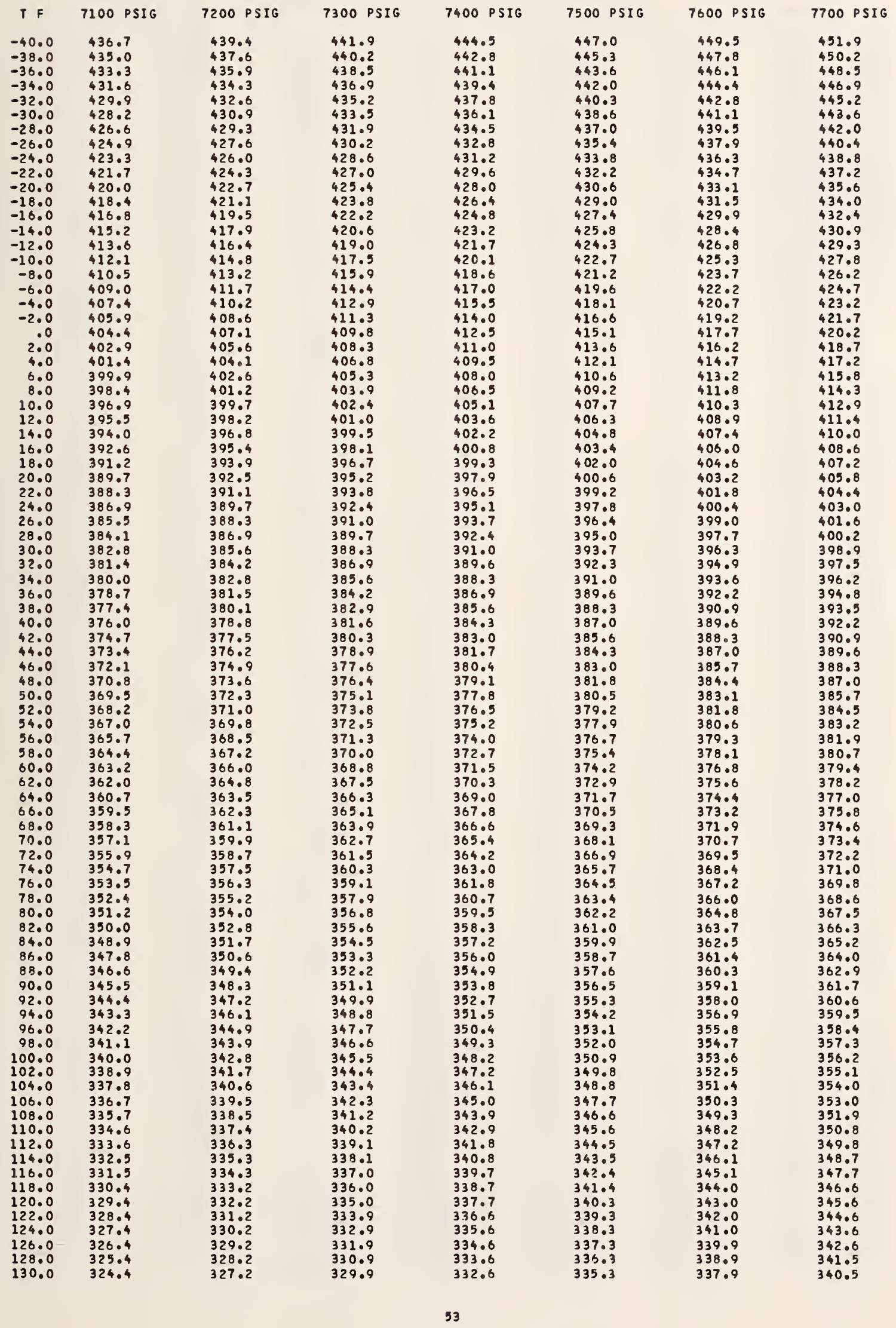


NITROGEN CONTENTS TABLE (SCF/CU FT).

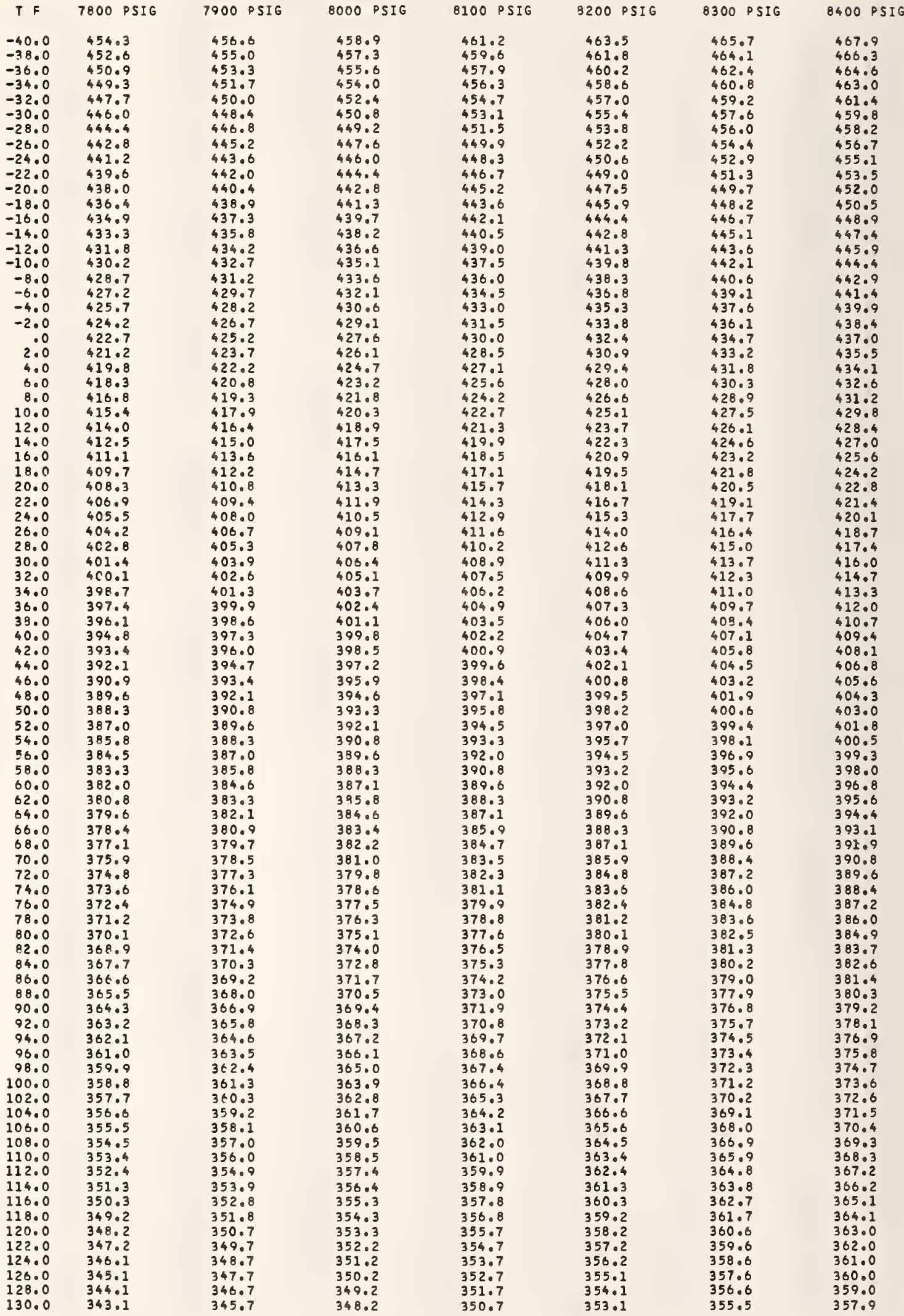


NITROGEN CONTENTS TABLE (SCF/CU FT).

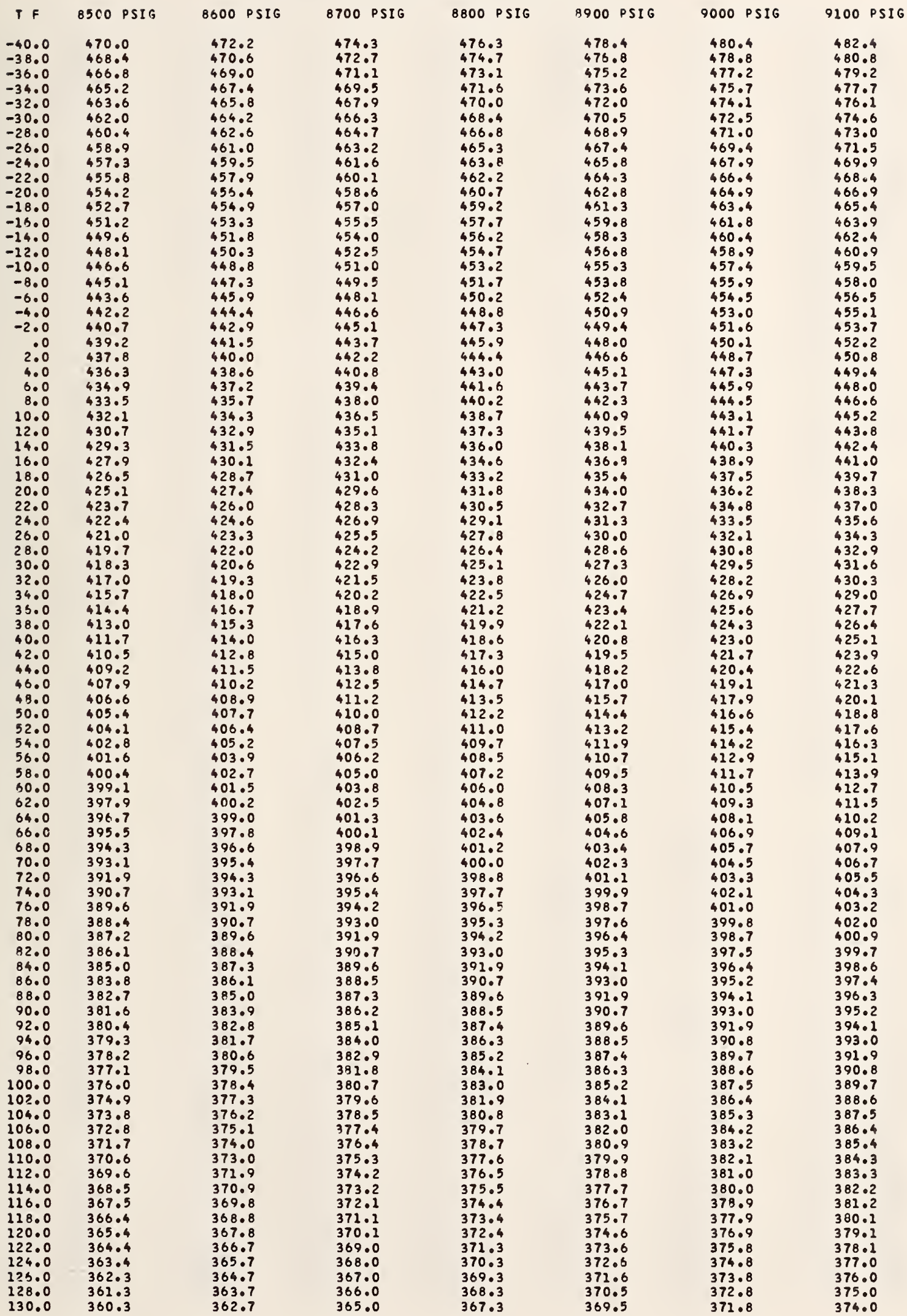


NITROGEN CONTENTS TABLE (SCF/CU FT).

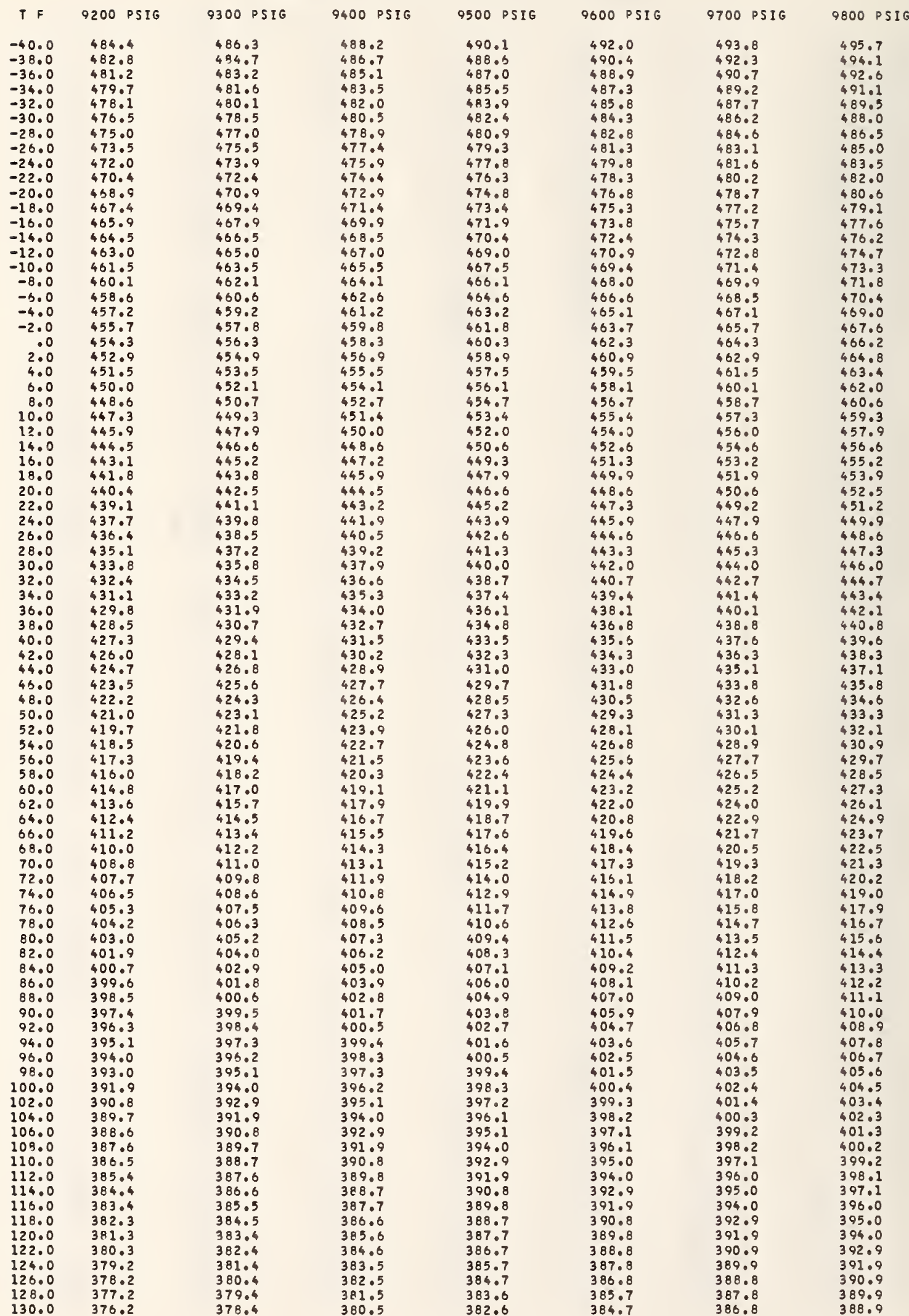


NITROGEN CONTENTS TABLE (SCFICU FT).

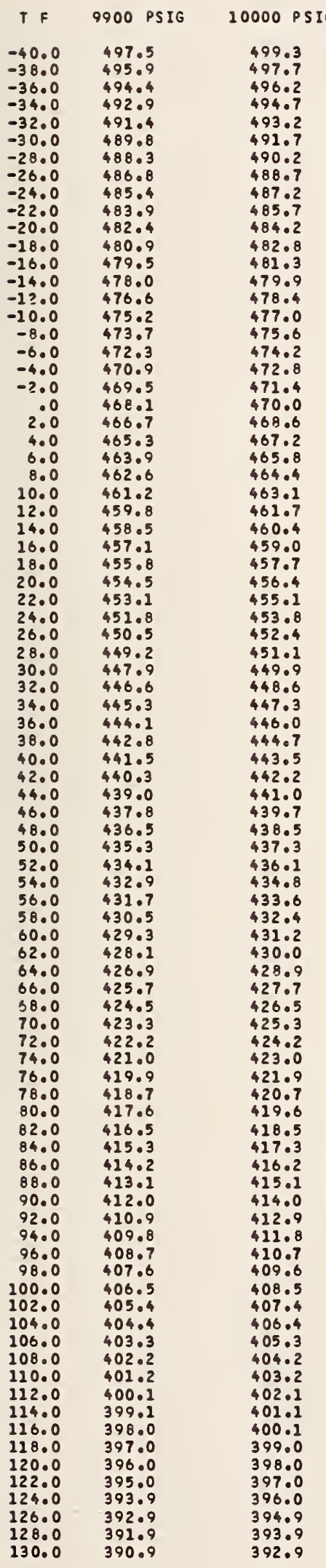


8.4

Helium Contents Table (SCF/cu ft) 
HELIUA CONTENTS TABLE (SCF/CU FT).

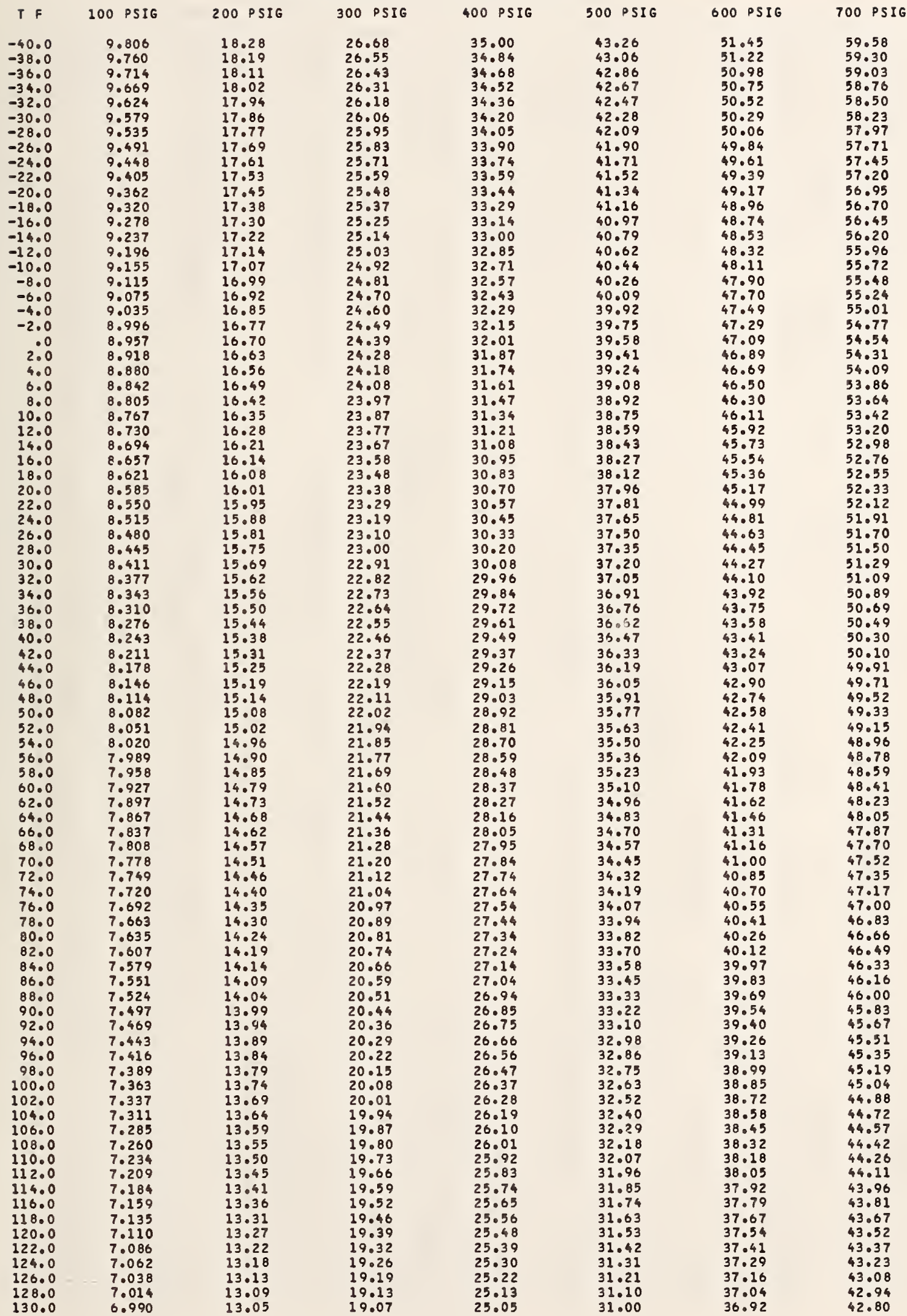


HELIUN CONTENTS TABLE (SCF/CU FT).

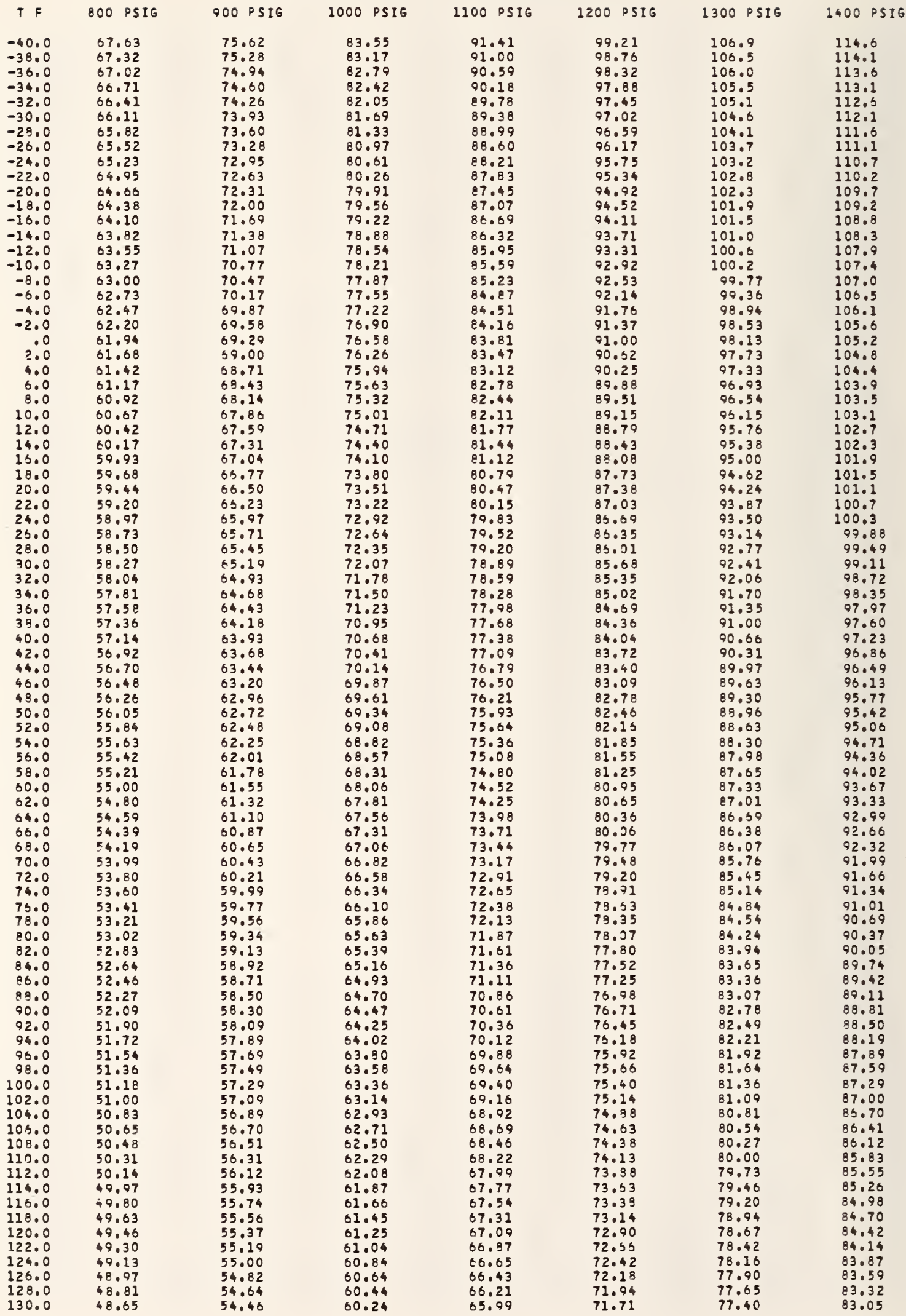


HELIUH CONTENTS TABLE (SCF/CU FT).

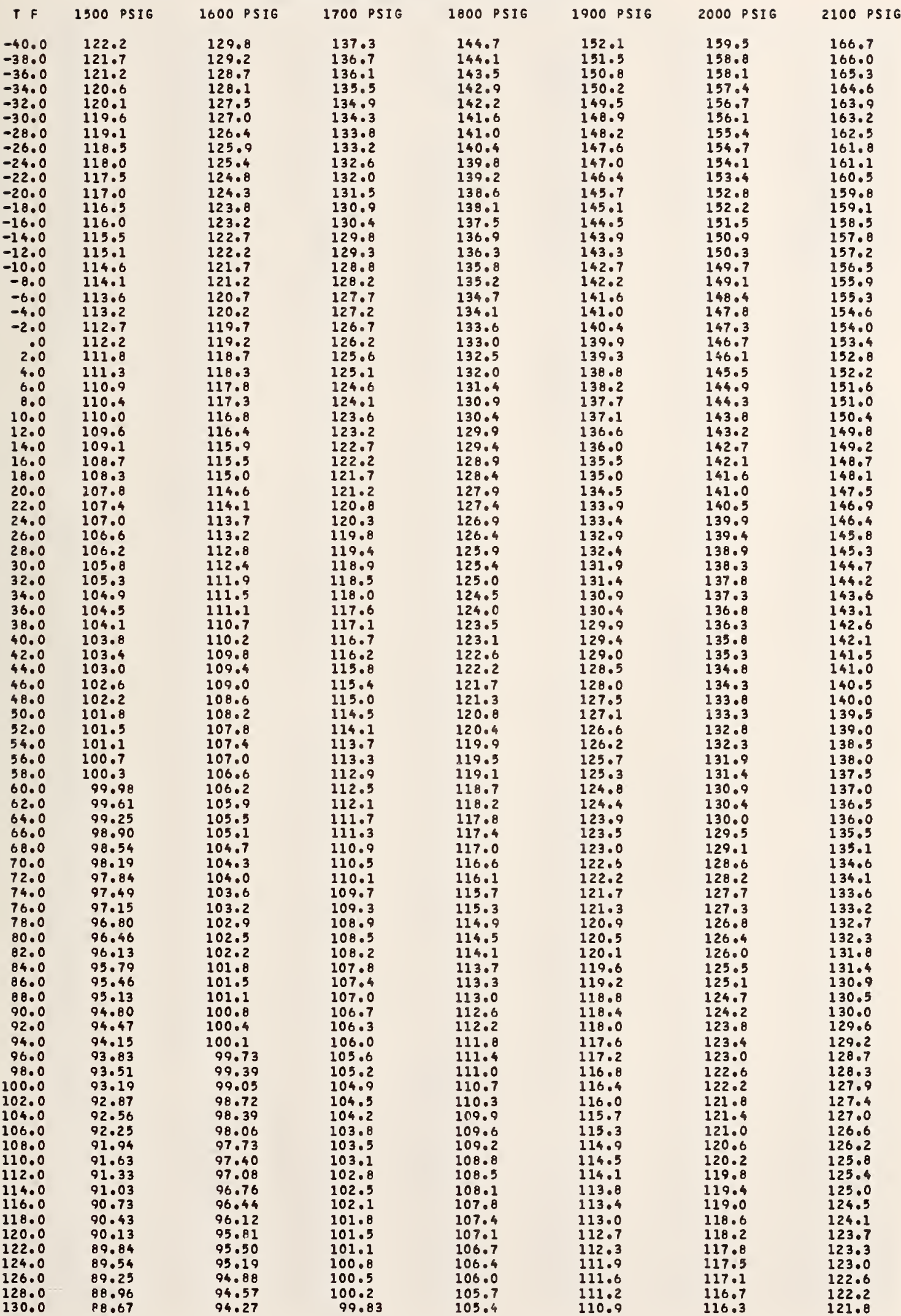




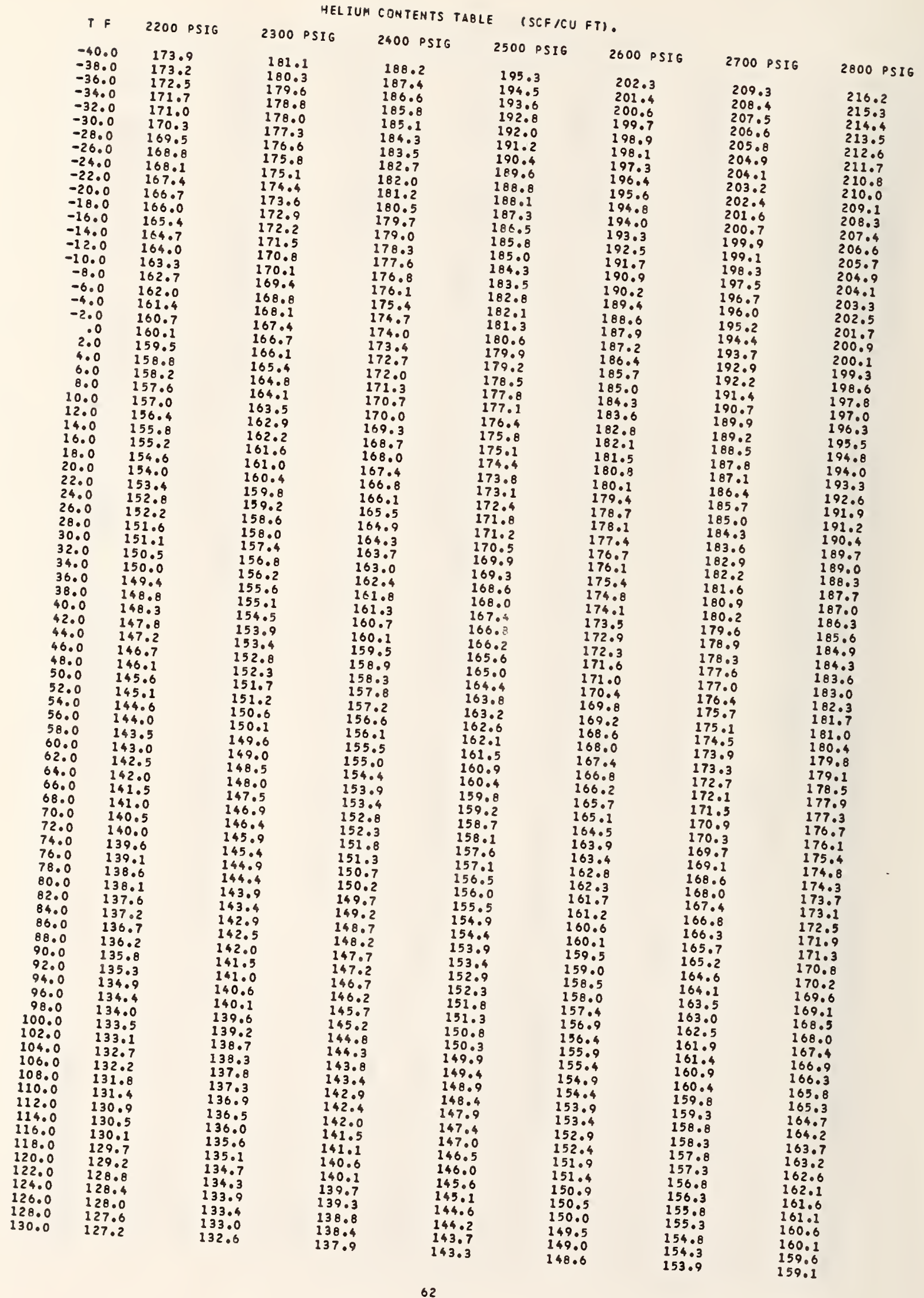


HELIUH CONTENTS TABLE (SCF/CU FT).

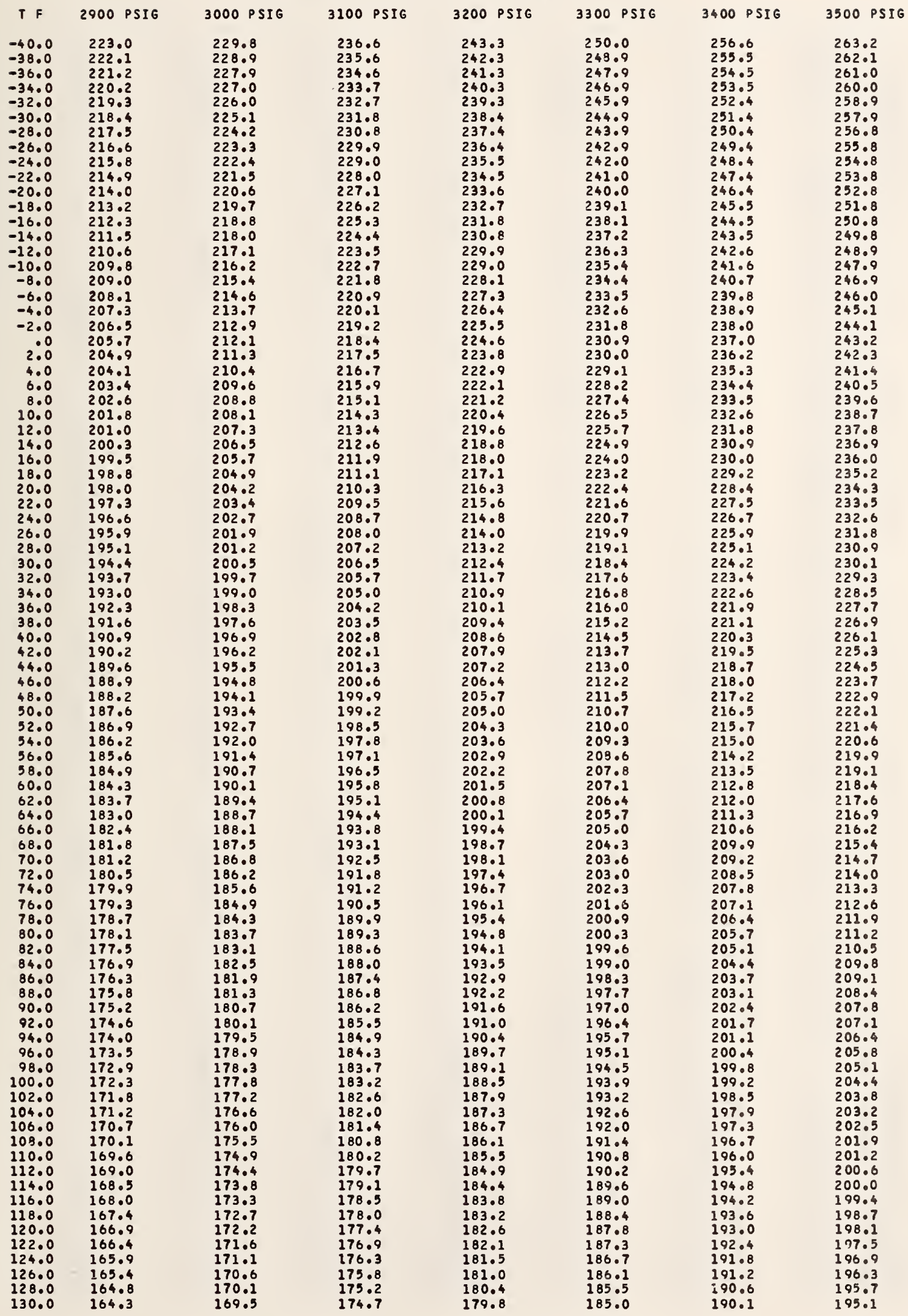


HELIUM CONTENTS TABLE (SCF/CU FT).

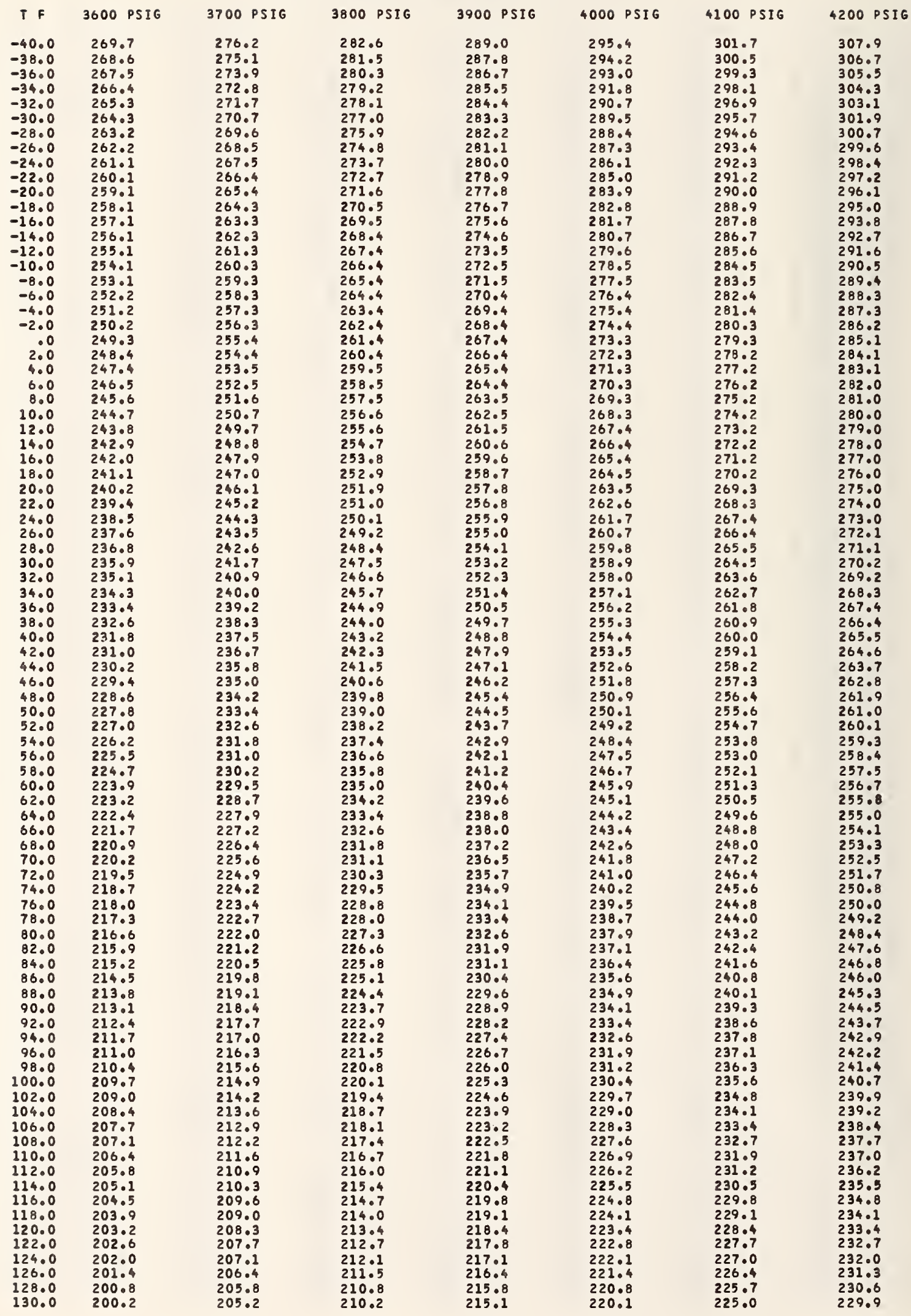


HELIUM CONTENTS TABLE (SCF/CU FT).

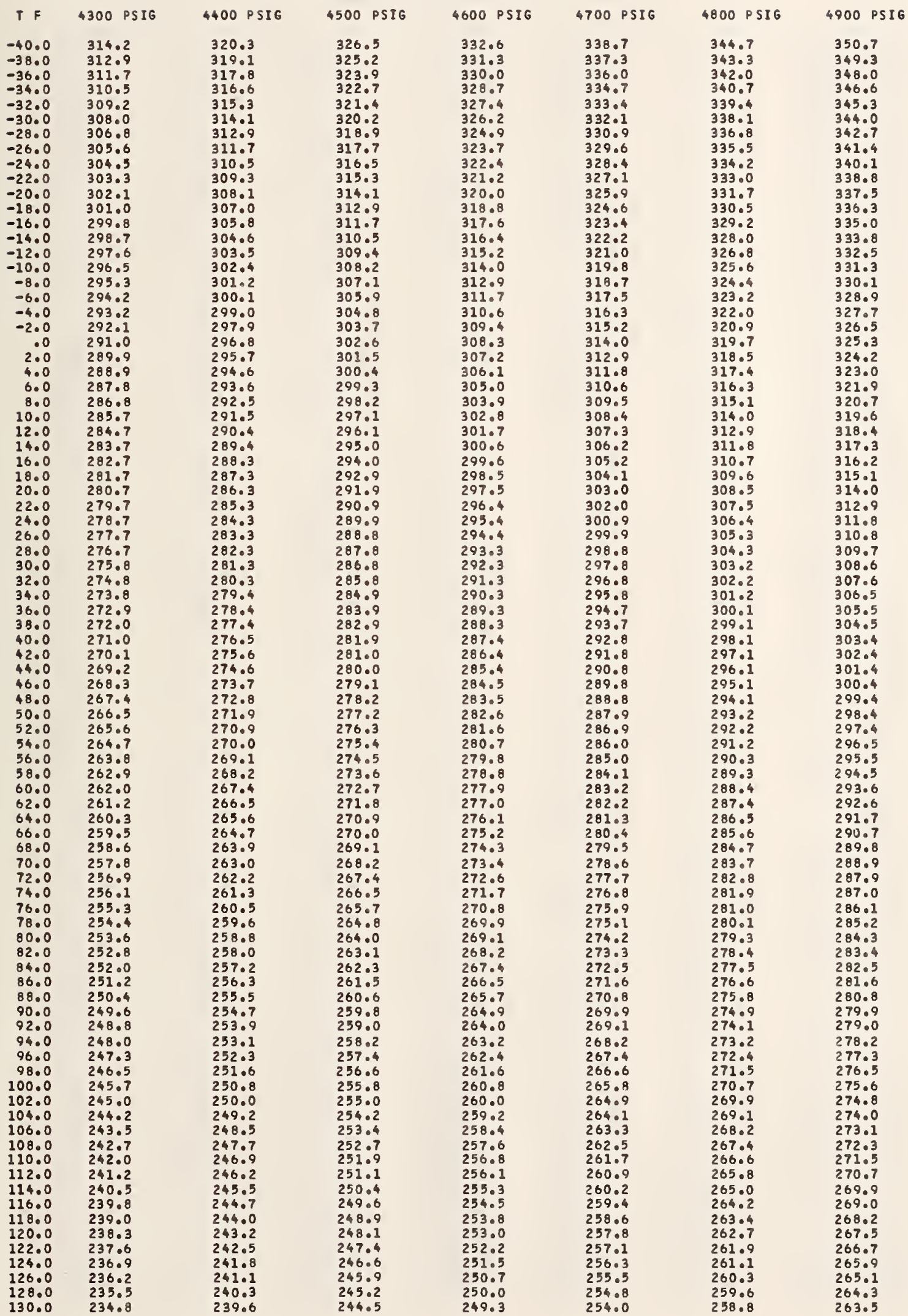


HELIUM CONTENTS TABLE (SCFICU FT).

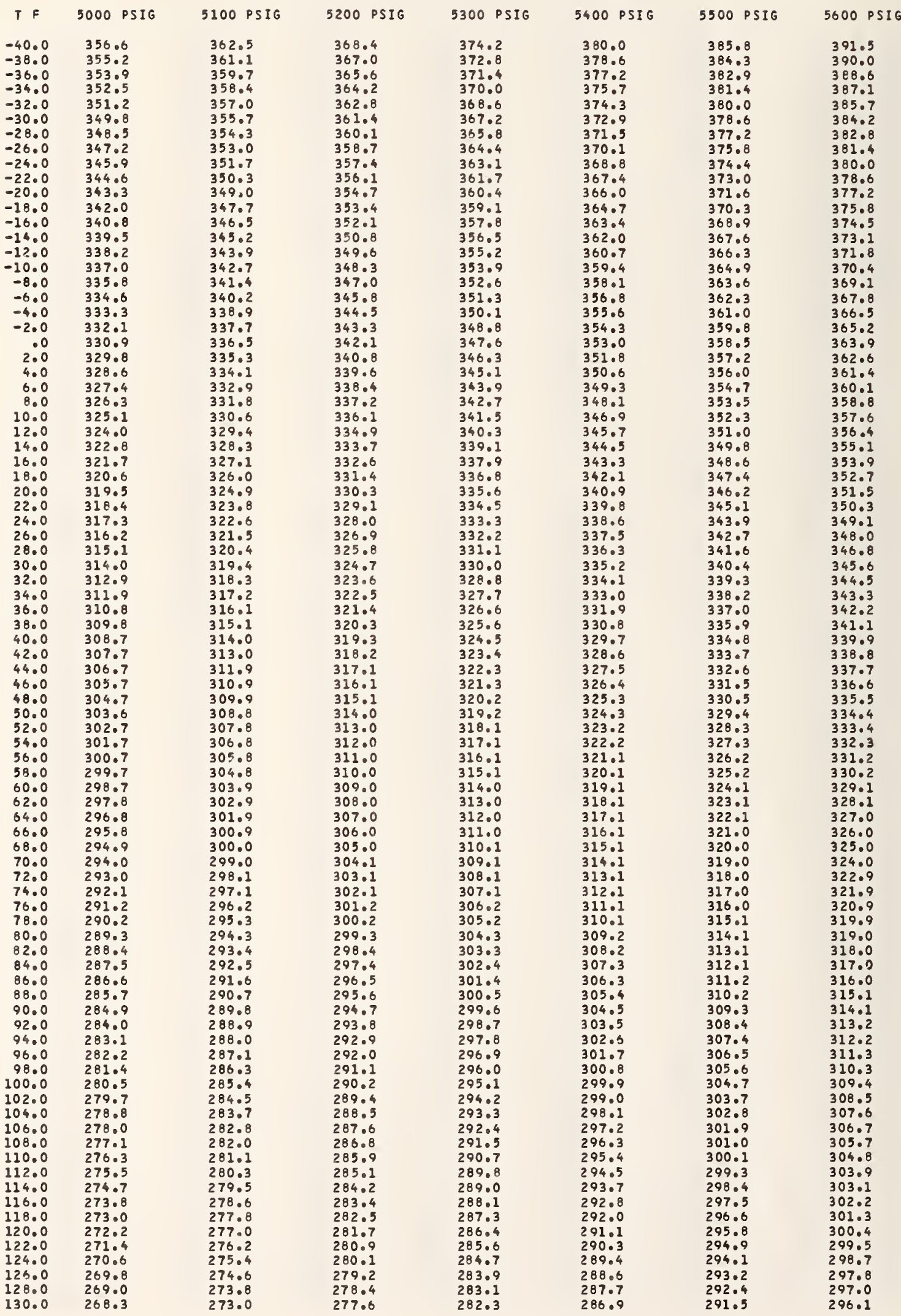


HELIUM CONTENTS TABLE (SCF/CU FT).

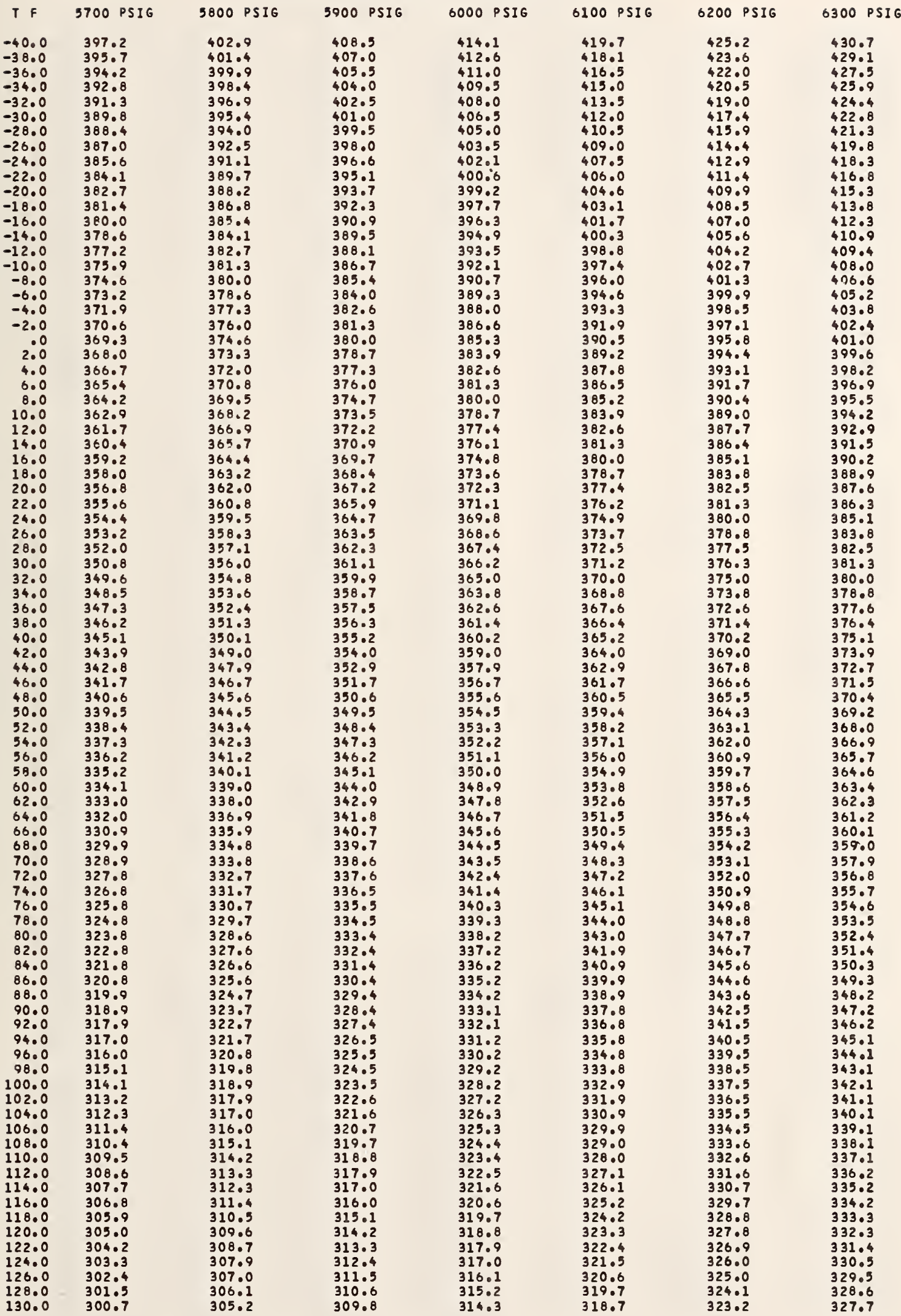


HELIUM CONTENTS TABLE (SCF/CU FT).

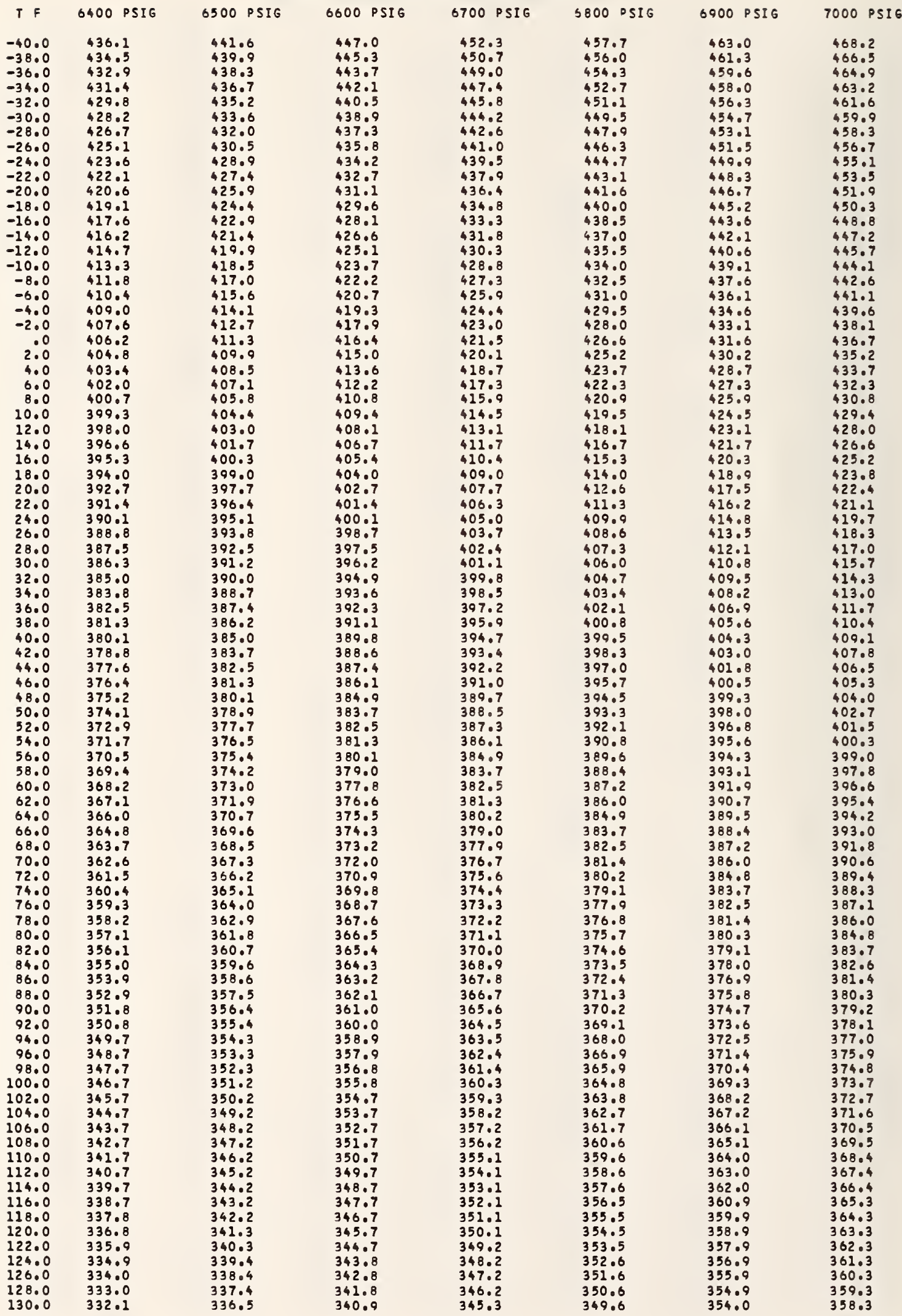


HELIUH CONTENTS TABLE (SCF/CU FT).

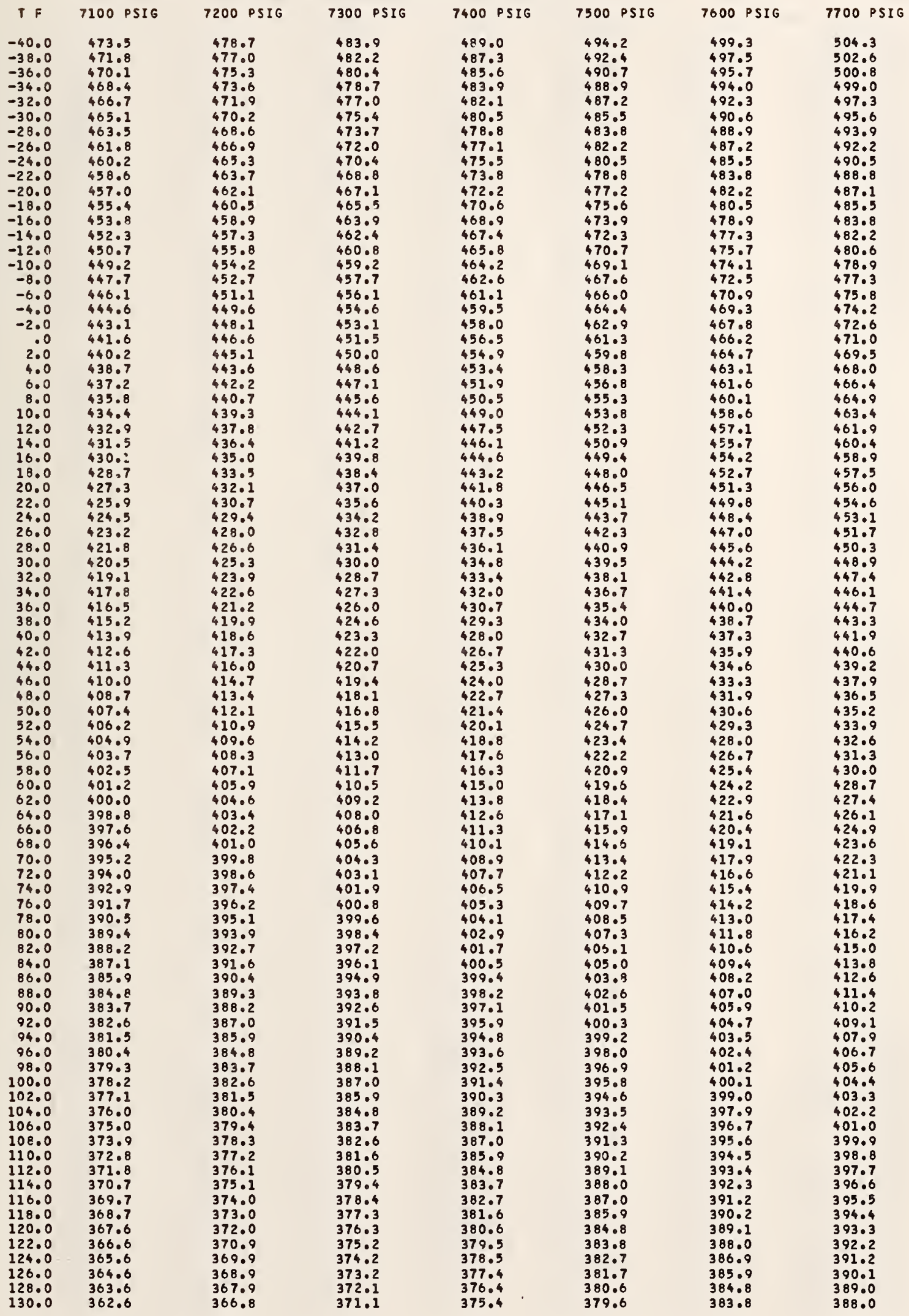


HEL IUM CONTENTS TABLE (SCF/CU FT).

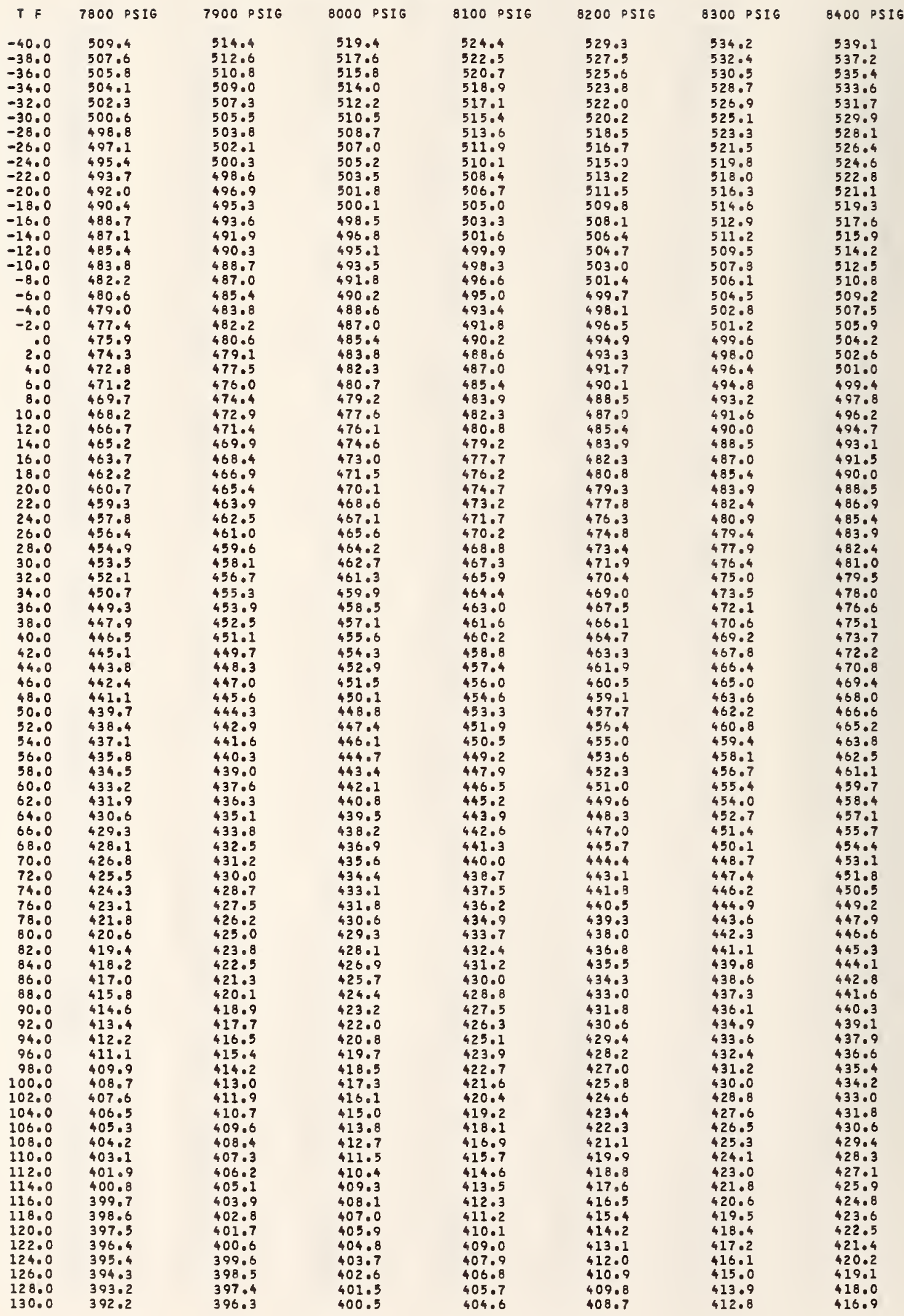


HELIUM CONTENTS TABLE (SCFICU FT).

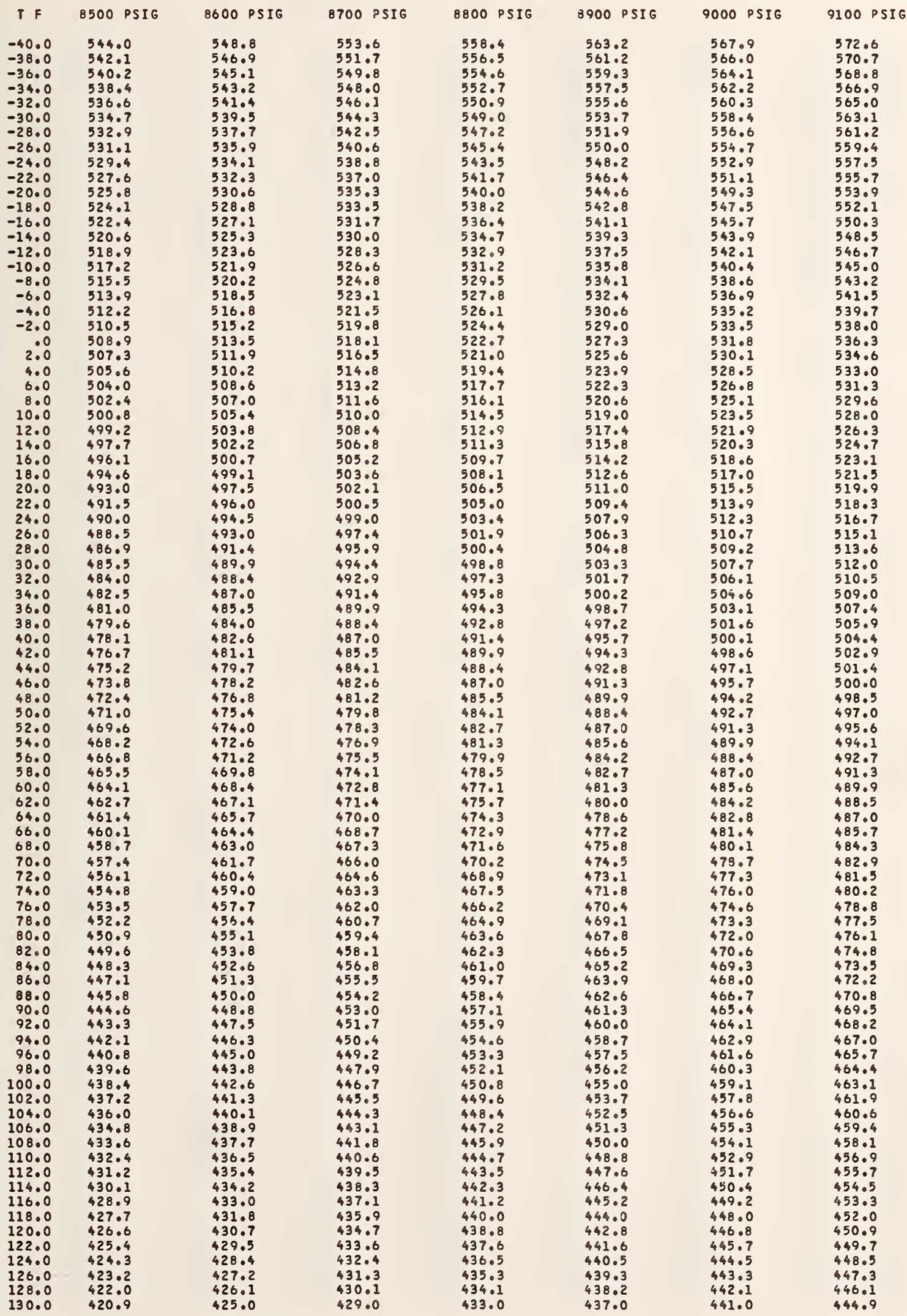


HELIUM CONTENTS TABLE (SCF/CU FT).

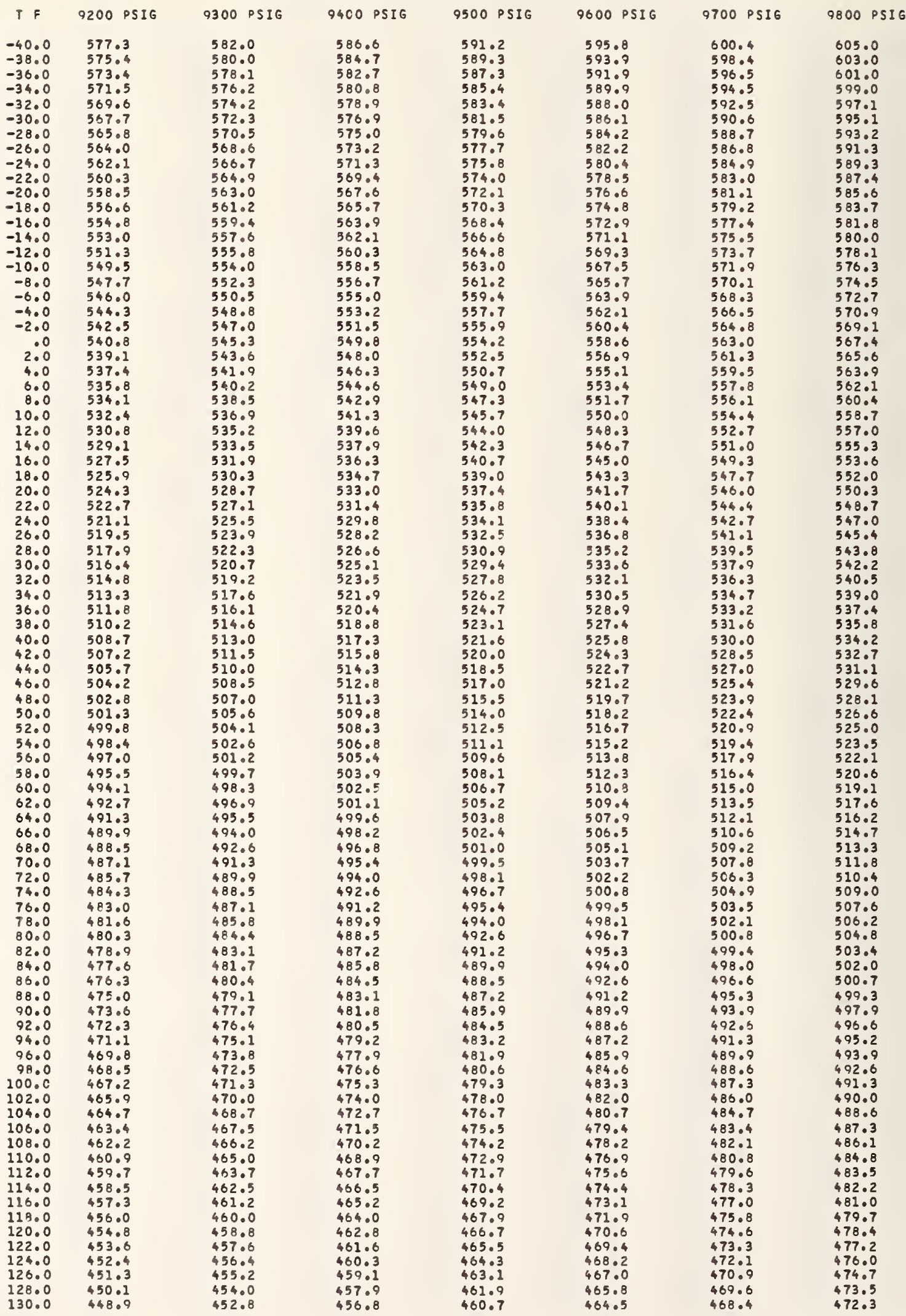


HELIUH CONTENTS TAQLE (SCF/CU FT).

TF 9900 PSIG 10000 PSIG

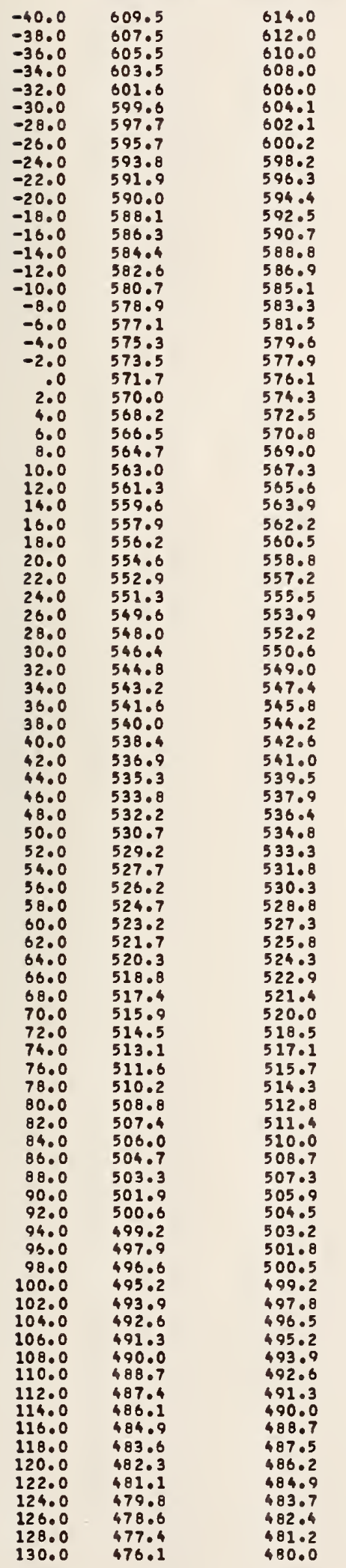


8.5

Hydrogen Contents Table (SCF/cu ft) 
HYOROGEN CONTENTS TABLE (SCF/CU FT).

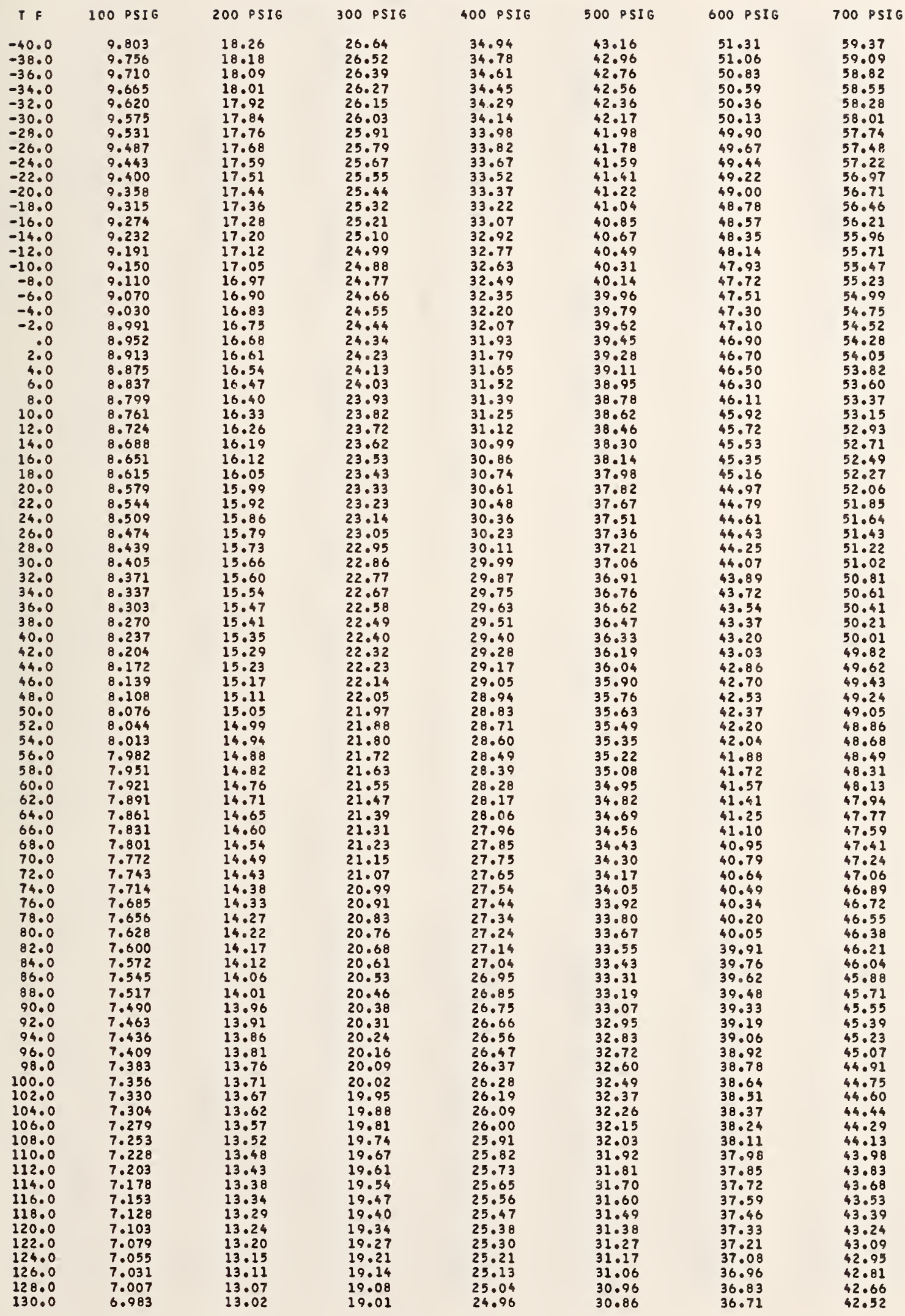


HYDROGEN CONTENTS TABLE (SCF/CU FT).

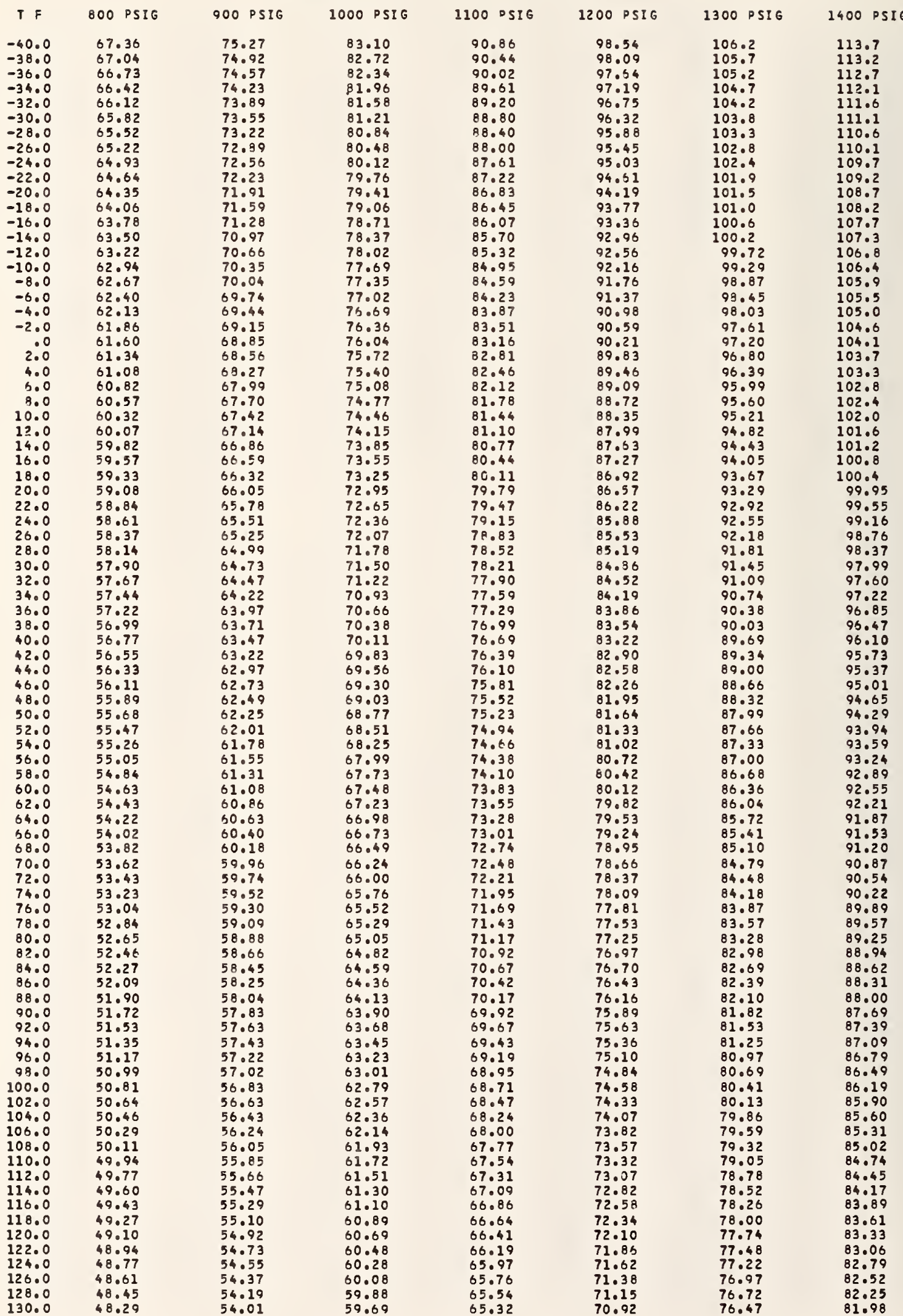


HYOROGEN CONTENTS TABLE (SCFICU FT).

-40
-38
-36

121.1

\section{6}

$-34.0 \quad 120.0$

$-32.0$

119.0

$\begin{array}{ll}-30.0 & 118.4 \\ -28.0 & 117.9\end{array}$

-26.0
-24.0

117.4

$-22.0 \quad 116.9$

$\begin{array}{ll}-20.0 & 116.3 \\ -18.0 & 115.8\end{array}$

$\begin{array}{ll}-18.0 & 115.3 \\ -16.0 & 114.0\end{array}$

$\begin{array}{ll}-16.0 & 114.8 \\ -14.0 & \end{array}$

$\begin{array}{ll}-12.0 & 114.3\end{array}$

$-10.0$

$-8.0$

113.9

113.4

$-4.0 \quad 112.4$

$-2.0 \quad 111.9$

$2.0 \quad 111.0$

$2.0 \quad 110.5$

$6.0 \quad 110.1$

$\begin{array}{rr}8.0 & 109.2 \\ 10.0 & 108.7\end{array}$

$12.0 \quad 108.3$

$14.0 \quad 107.8$

$16.0 \quad 107.4$

$18.0 \quad 107.0$

$20.0 \quad 106.6$

$\begin{array}{ll}22.0 & 106.1 \\ 24.0 & 105.7\end{array}$

$26.0 \quad 105.3$

$28.0 \quad 104.9$

$30.0 \quad 104.5$

$\begin{array}{ll}32.0 & 104.1 \\ 34.0 & 103.7\end{array}$

$36.0 \quad 103.3$

$38.0 \quad 103.3$

$40.0 \quad 102.5$

$\begin{array}{ll}42.0 & 102.1 \\ 44.0 & 101.7\end{array}$

48.0

50.0

101.3

100.9

100.5

$\begin{array}{cc}54.0 & 100.2 \\ 59.0 & 99.79\end{array}$

56.0

58.0

60.0

62.0

64.0

66.0

70.0

72.0

72.0
74.0

76.0

79.0

80.0

82.0

84.0

86.0

88.0

90.0

92.0

94.0

96.0

100.0

102.0

102.0
104.0

104.0

108.0

110.0

112.0

114.0

116.0

118.0

120.0

122.0

124.0

126.0

128.0

130.0

$\begin{array}{ll}99.79 & 105.9 \\ 99.42 & 105.5 \\ 99.05 & 105.2 \\ 98.68 & 104.8 \\ 98.32 & 104.4 \\ 97.96 & 104.0 \\ 97.61 & 103.6 \\ 97.25 & 103.3 \\ 96.90 & 102.9 \\ 96.55 & 102.5 \\ 96.21 & 102.1 \\ 95.86 & 101.8 \\ 95.52 & 101.4 \\ 95.18 & 101.1 \\ 94.85 & 100.7 \\ 94.51 & 100.4 \\ 94.18 & 100.0 \\ 93.85 & 99.66 \\ 93.53 & 99.31 \\ 93.20 & 98.97 \\ 92.88 & 98.63 \\ 92.56 & 98.29 \\ 92.24 & 97.95 \\ 91.93 & 97.62 \\ 91.61 & 97.29 \\ 91.30 & 96.96 \\ 90.99 & 96.63 \\ 90.69 & 96.30 \\ 90.38 & 95.98 \\ 90.08 & 95.66 \\ 9.78 & 95.34 \\ 9.48 & 95.03 \\ 9.18 & 94.71 \\ 8.89 & 94.40 \\ 9.60 & 94.09 \\ 8.31 & 93.79 \\ 8.02 & 93.48 \\ 7.73 & 93.18 \\ 9.45 & 92.88 \\ & \\ 9.98 & \end{array}$

128.5

127.9
127.4

126.8

126.2
125.7

125.1

124.6
124.0

123.5

122.9

122.4

121.9

121.3

120.3

119.8

119.3

118.3

117.8

116.8

116.3

115.9

115.4

114.9

114.0

113.6

113.1
112.6

112.6

111.8

111.3

110.9

110.5

109.6

109.2

108.8

108.4
107.9

107.9
107.5

107.5
107.1

106.7

106.3

105.9
105.5

05.2

04.8

104.4

103.6

02.9

02.5

102.1

101.4

101.1

100.4

00.0

99.31

98.97

98.63
98.29

97.95

97.29

6.63

5.66

95.34

4.71

93.79

93.18

(1)

1700 PSIG

1800 PIG

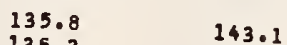

143.1

135.2

134.0

133.4

132.8

132.2
131.7

131.1

130.5
129.9

129.4

128.3

127.7
127.2

127.2

126.1

125.6

125.1

124.5

123.5

123.0

122.5

122.0

121.5

120.5

120.1

119.6

119.1

118.6

118.2

117.3

116.8

116.4

115.9

115.5

115.0

114.6

114.2

113.7

113.3

112.5

112.0

111.6

111.2

110.8

110.0

109.6

109.2

108.8

108.4

108.0

107.7

107.3

106.9

106.5

106.2

105.8

105.4

105.0

104.3

104.0

103.6

103.3

102.6

102.2

101.9

101.5

101.2

100.9

100.5

100.2
99.87

99.55

99.22

98.90

98.58
98.26

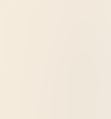

$\begin{array}{ll}143.1 & 150.3 \\ 142.4 & 149.6\end{array}$

2000 PSIG

2100 PSIG

141.2

140.5

139.9

139.3

138.1

136.9

136.3
135.7

135.7

134.6

134.6

133.4

132.9

132.3
131.8

131.2

130.7

130.1

129.1

128.6

128.0

127.5

127.0

126.5

126.0
125.5

125.5

125.0

124.1

123.6
123.1

123.1

122.2

121.7

121.2

120.3

119.9

119.4

119.0

118.5

118.1

117.7

117.2

116.8
116.4

115.9

115.5

115.1

114.7

114.3

113.5

113.1

112.7

112.3

111.9

111.5

111.1
110.7

110.7

110.0

109.6

109.2

108.9

108.5

107.8

107.4

107.1

106.7

106.0

105.7 


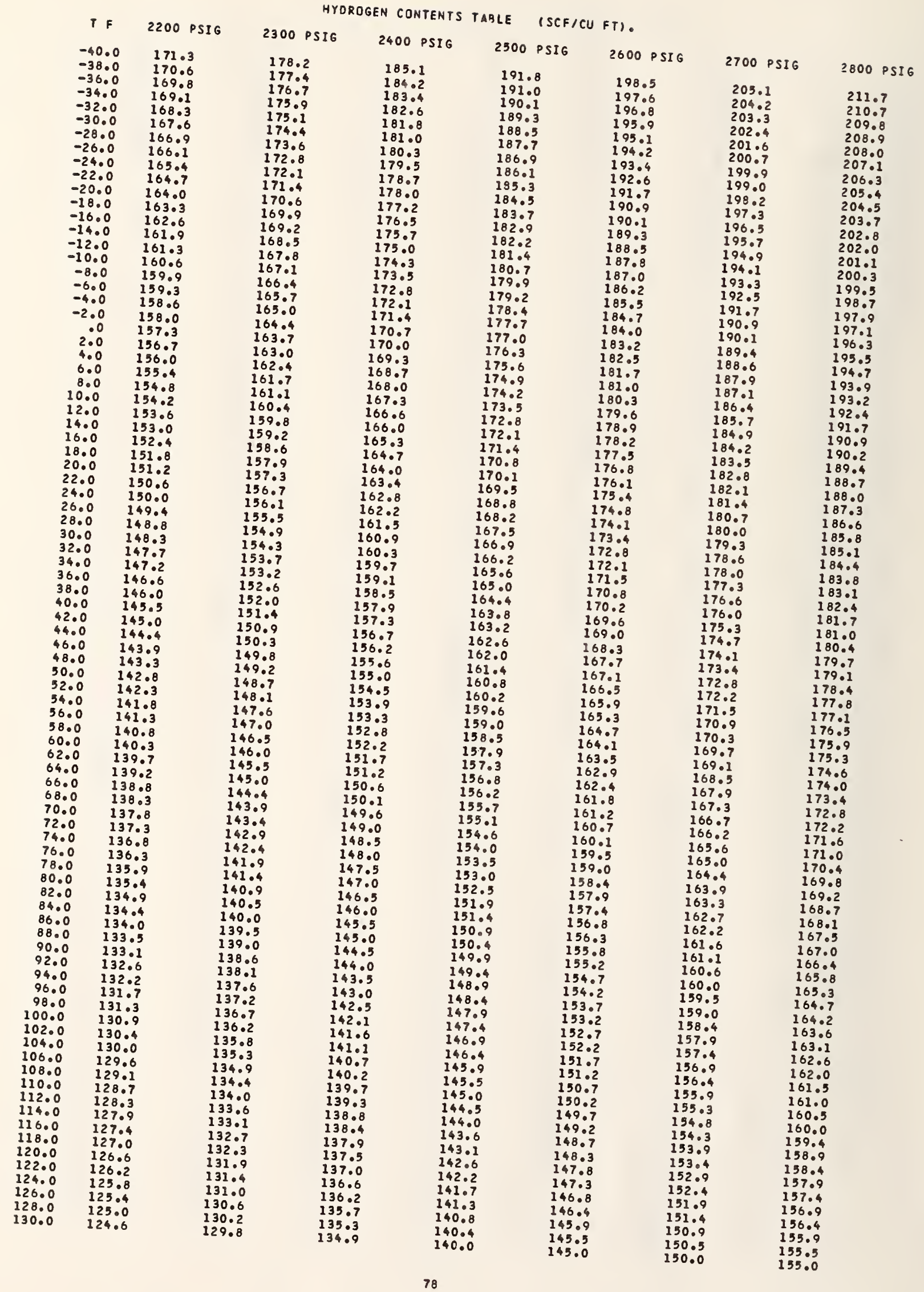


MYDROGEN CONTENTS TABLE (SCF/CU FT).

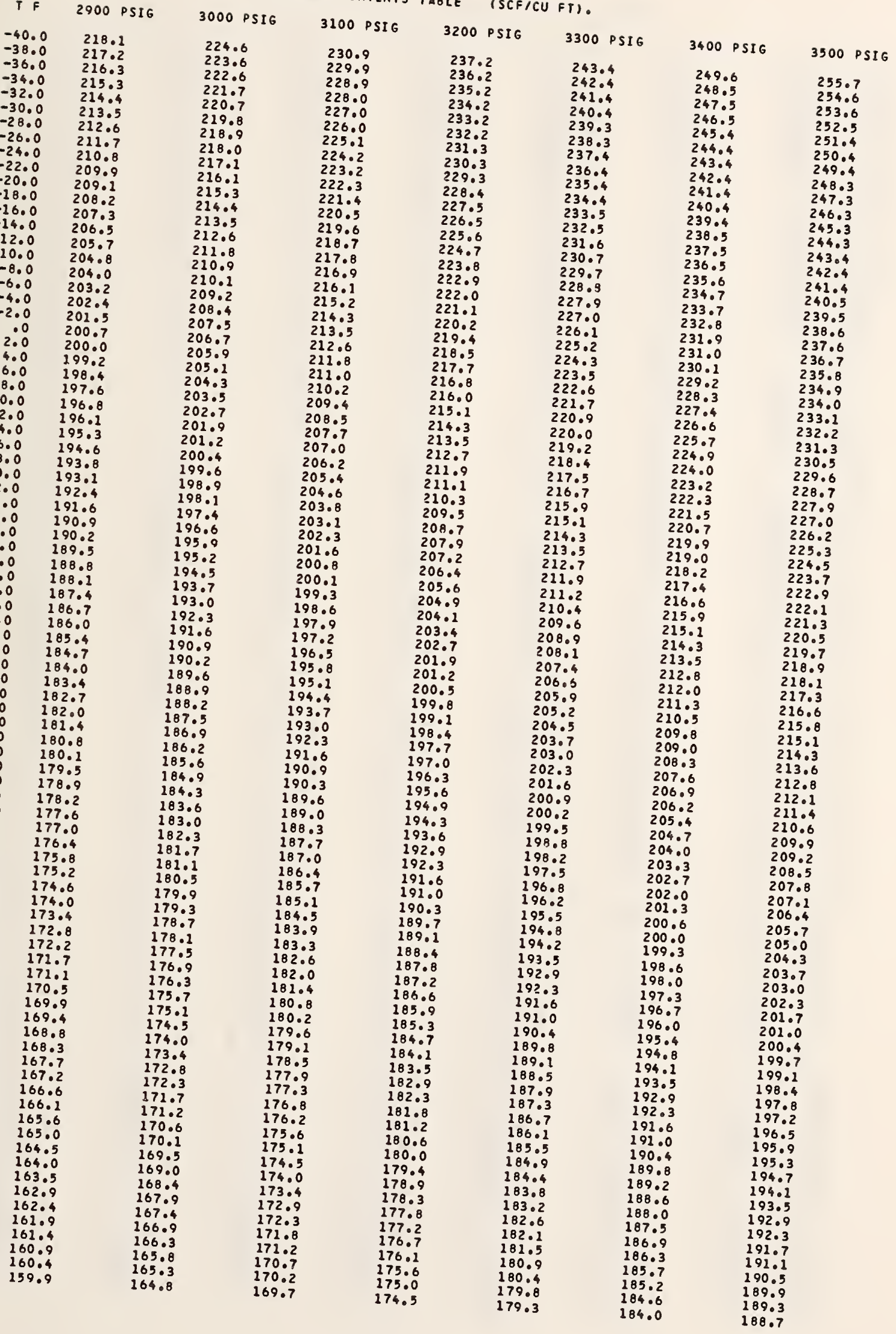


HYOROGEN CONTENTS TABLE (SCF/CU FT).

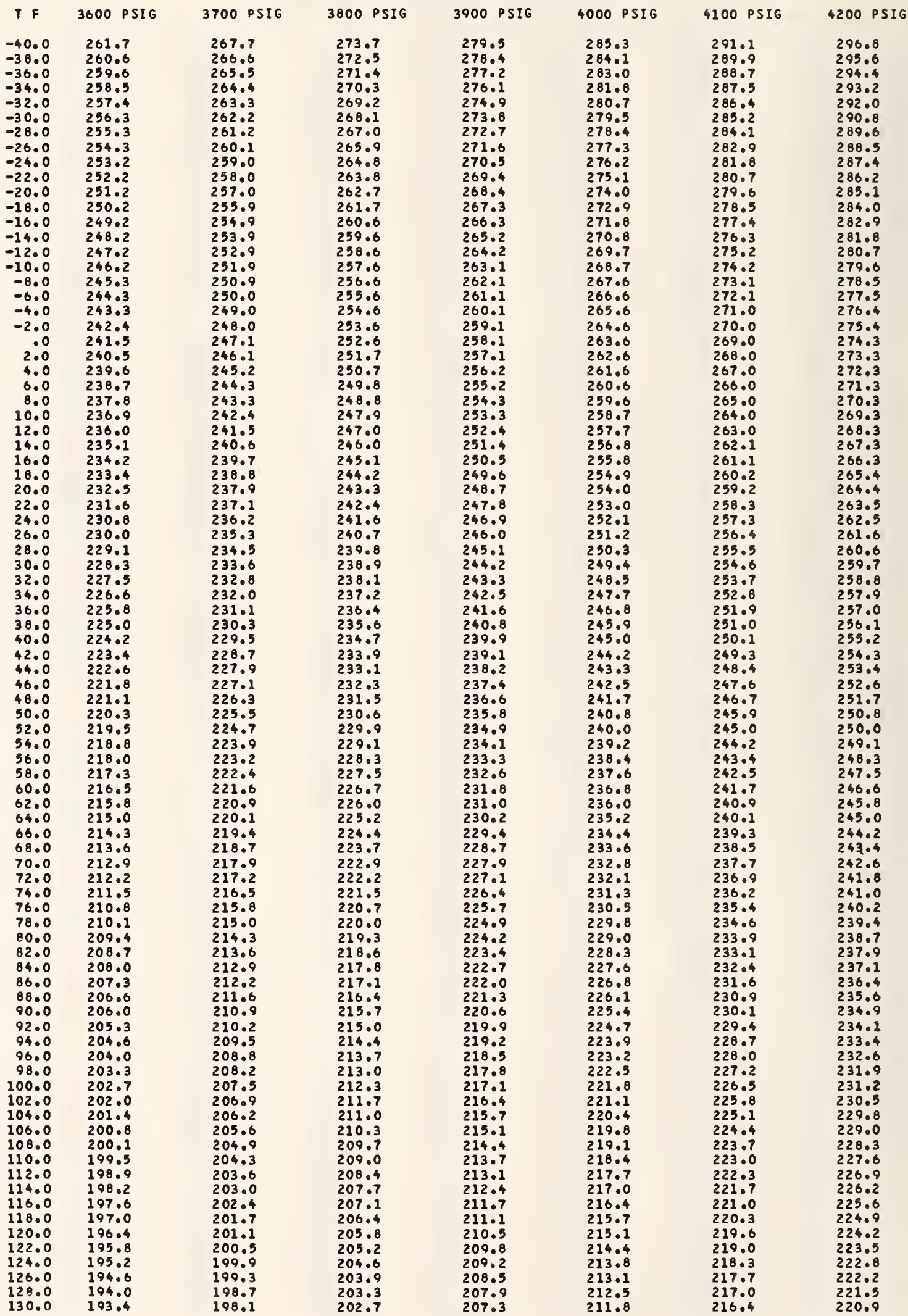


HYDROGEN CONTENTS TABLE (SCF/CU FT).

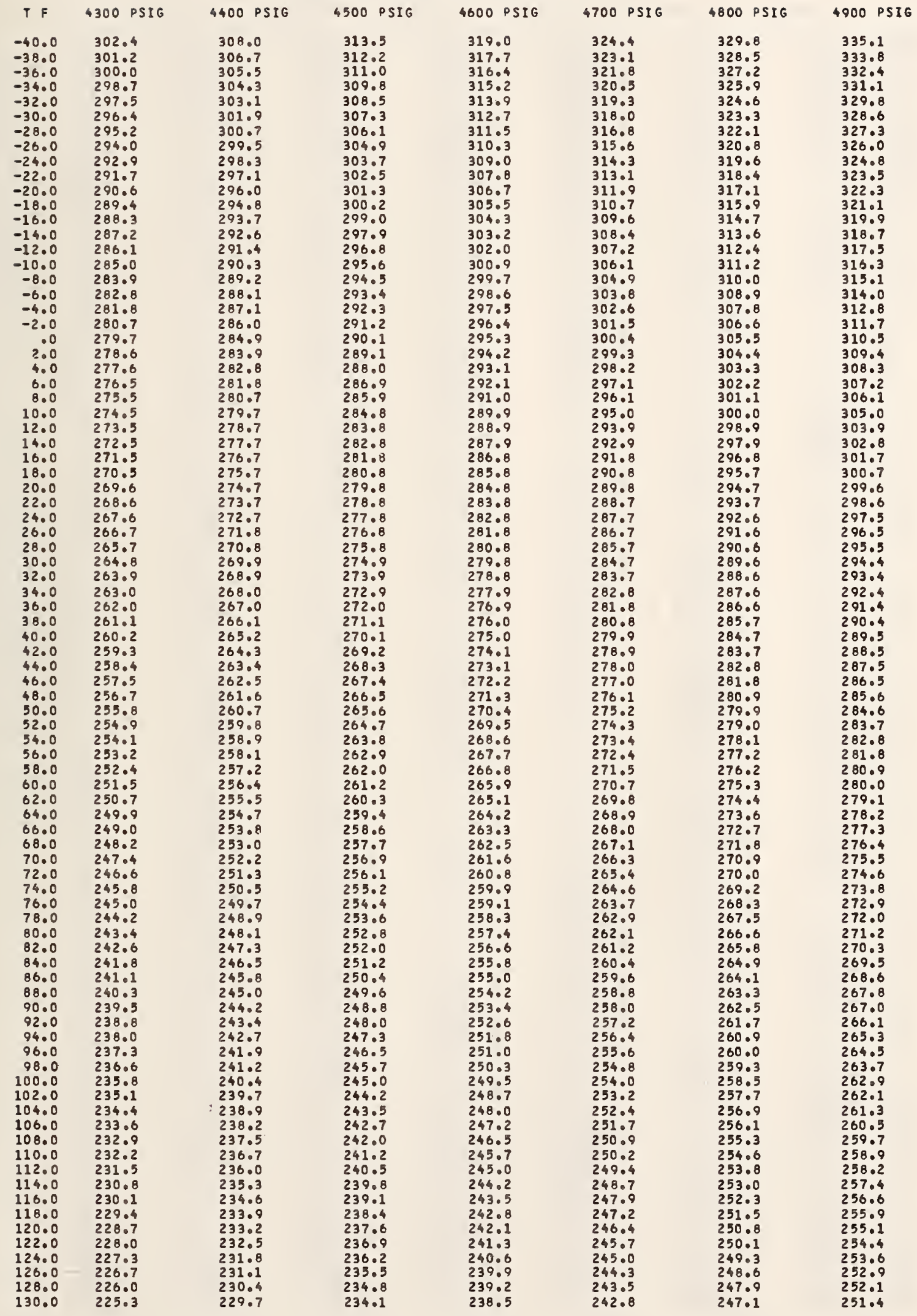


HYDROGEN CONTENTS TABLE (SCF/CU FT).

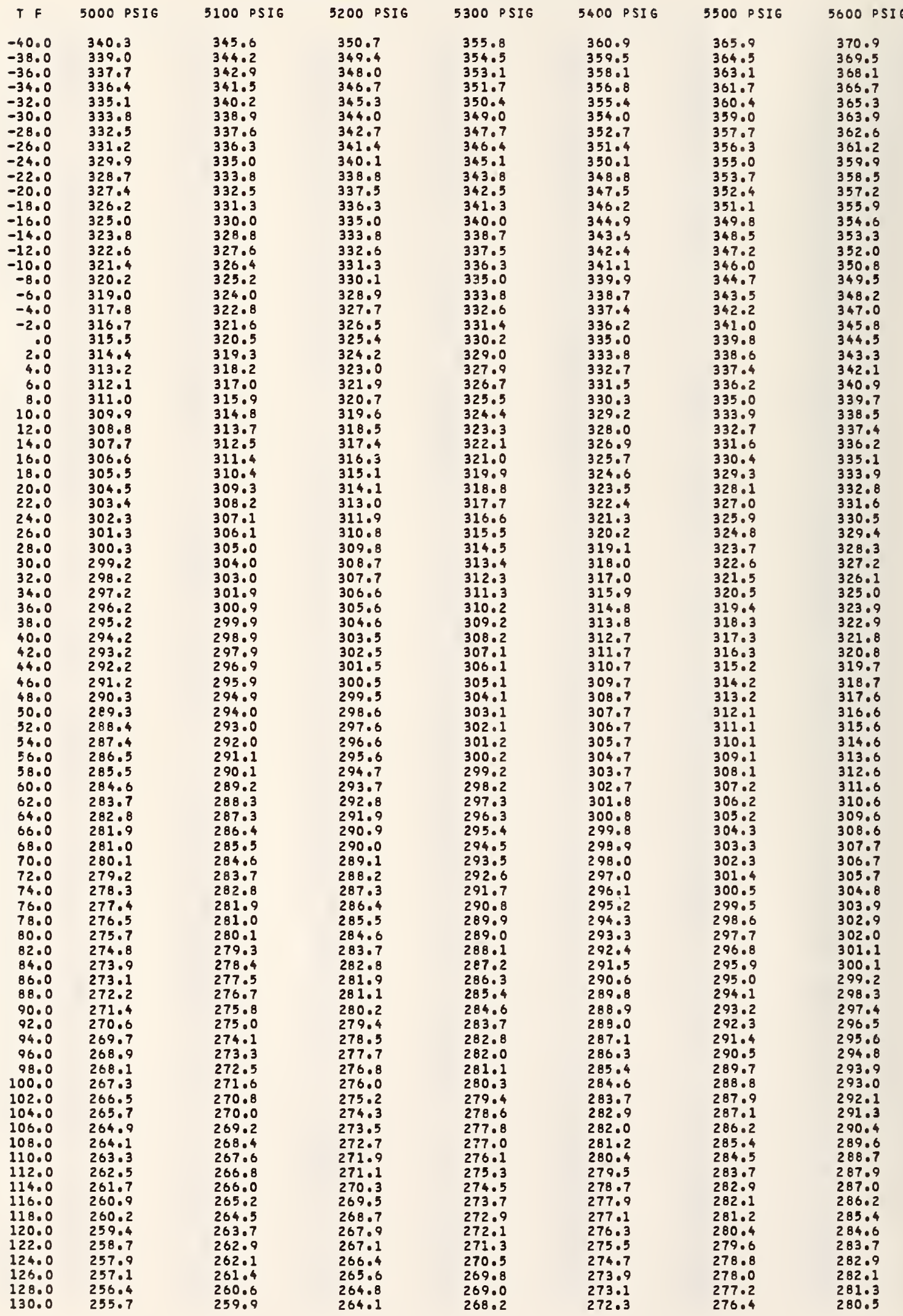


HYOROGEN CONTENTS TABLE (SCF/CU FT).

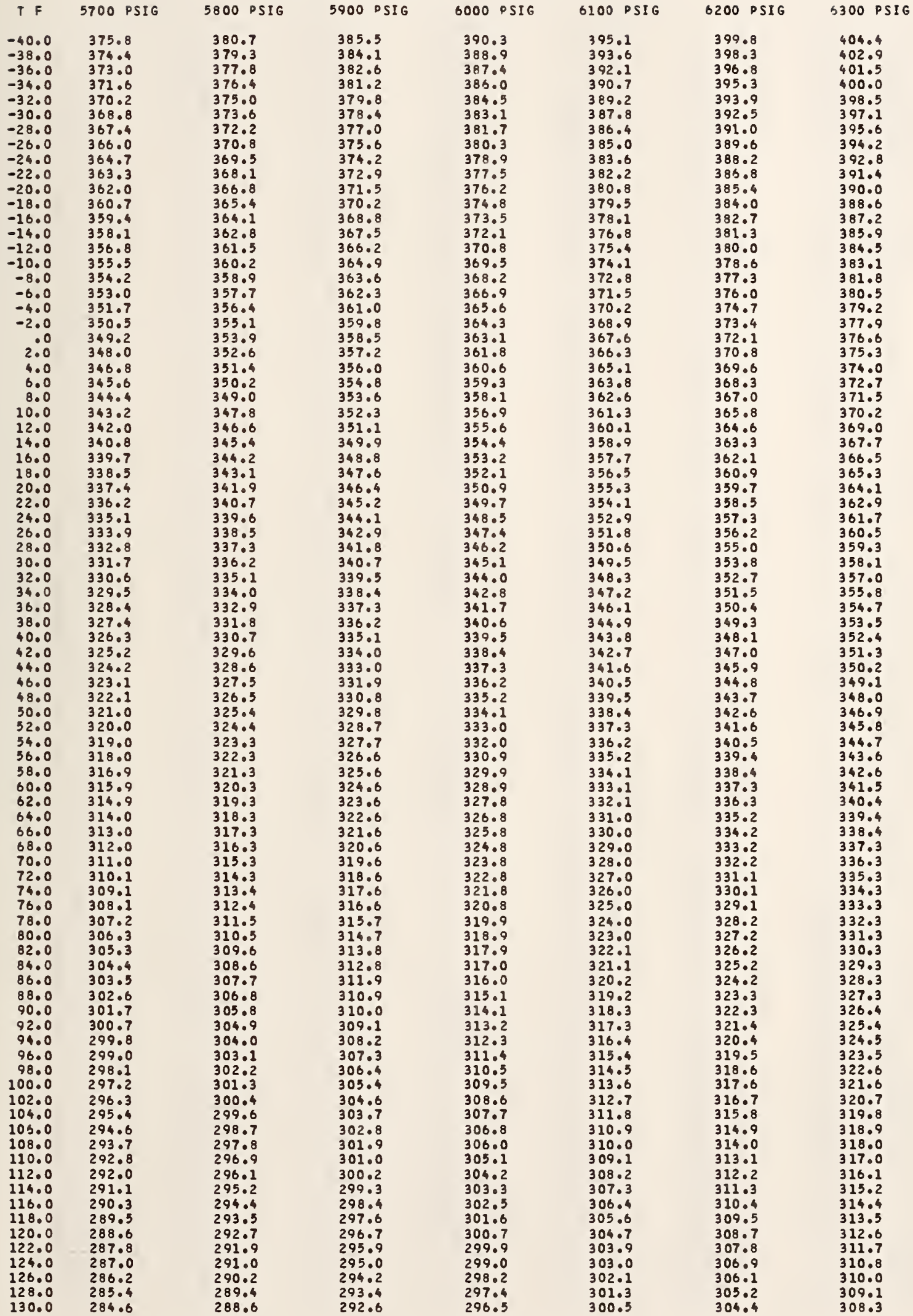


HYDRQGEN CONTENTS TABLE (SCF/CU FT).

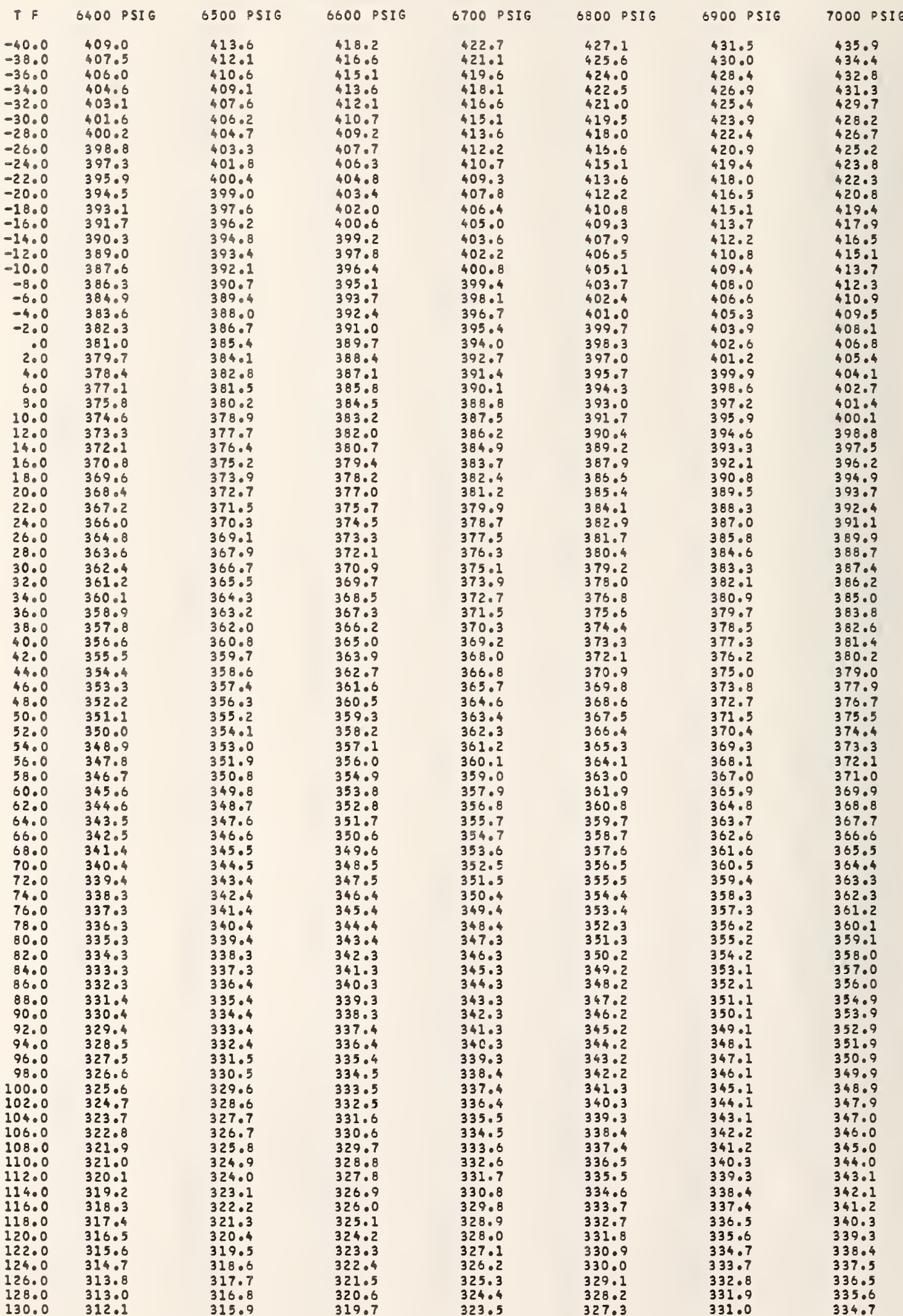


HYDROGEN CONTENTS TABLE (SCF/CU FT).

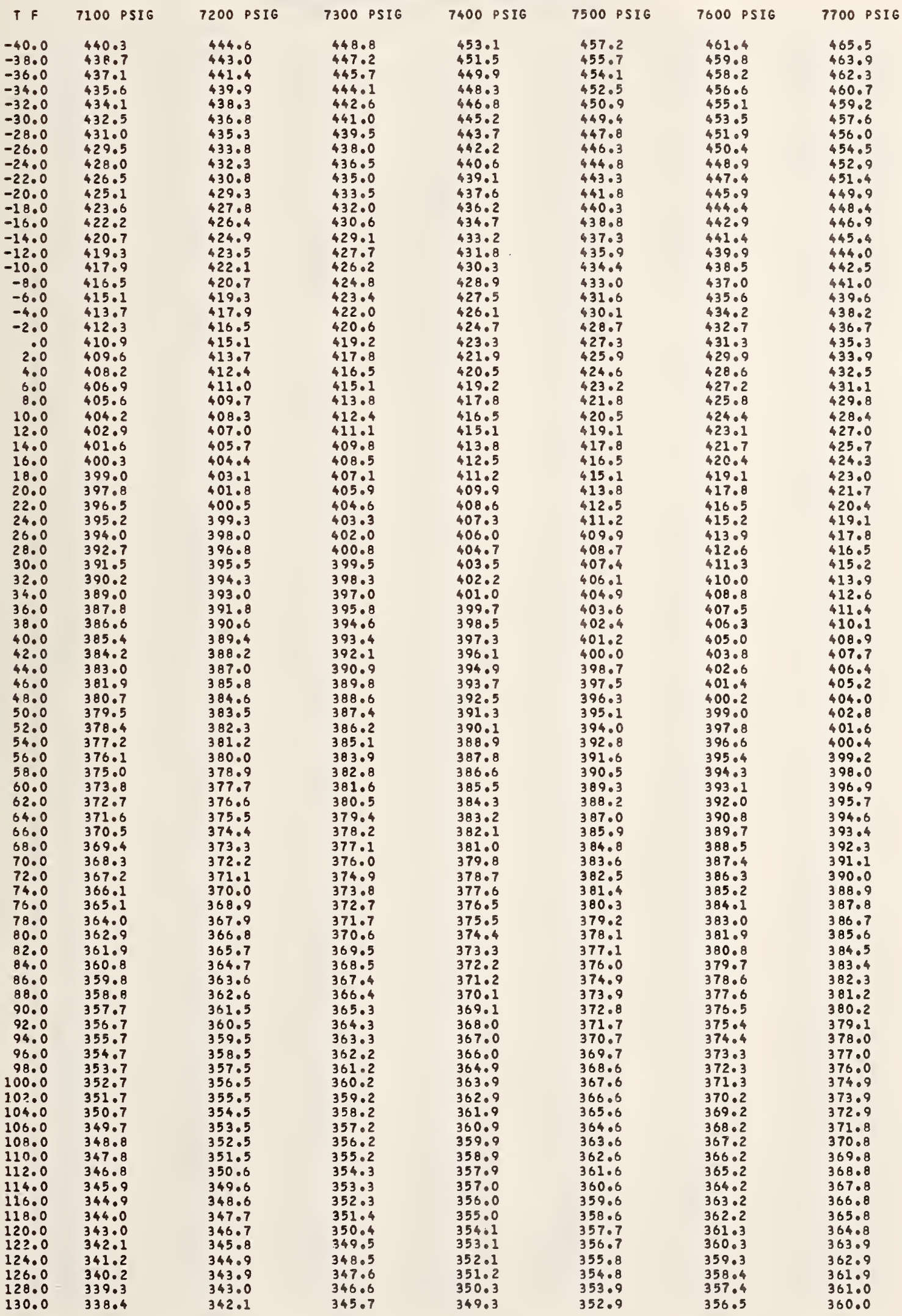


HYDROGEN CONTENTS TABLE (SCF/CU FT).

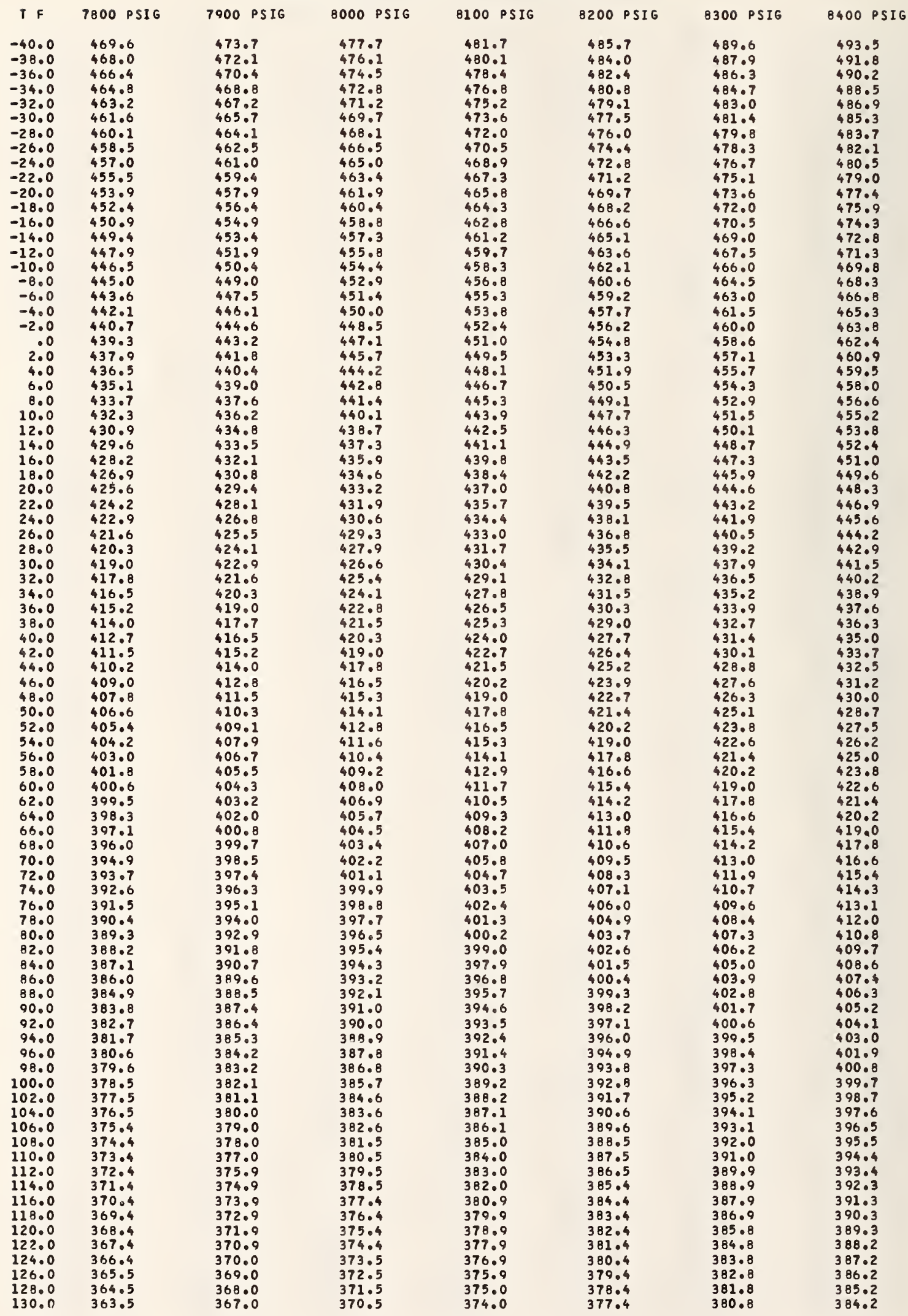


HYOROGEN CONTENTS TABLE (SCFICU FT).

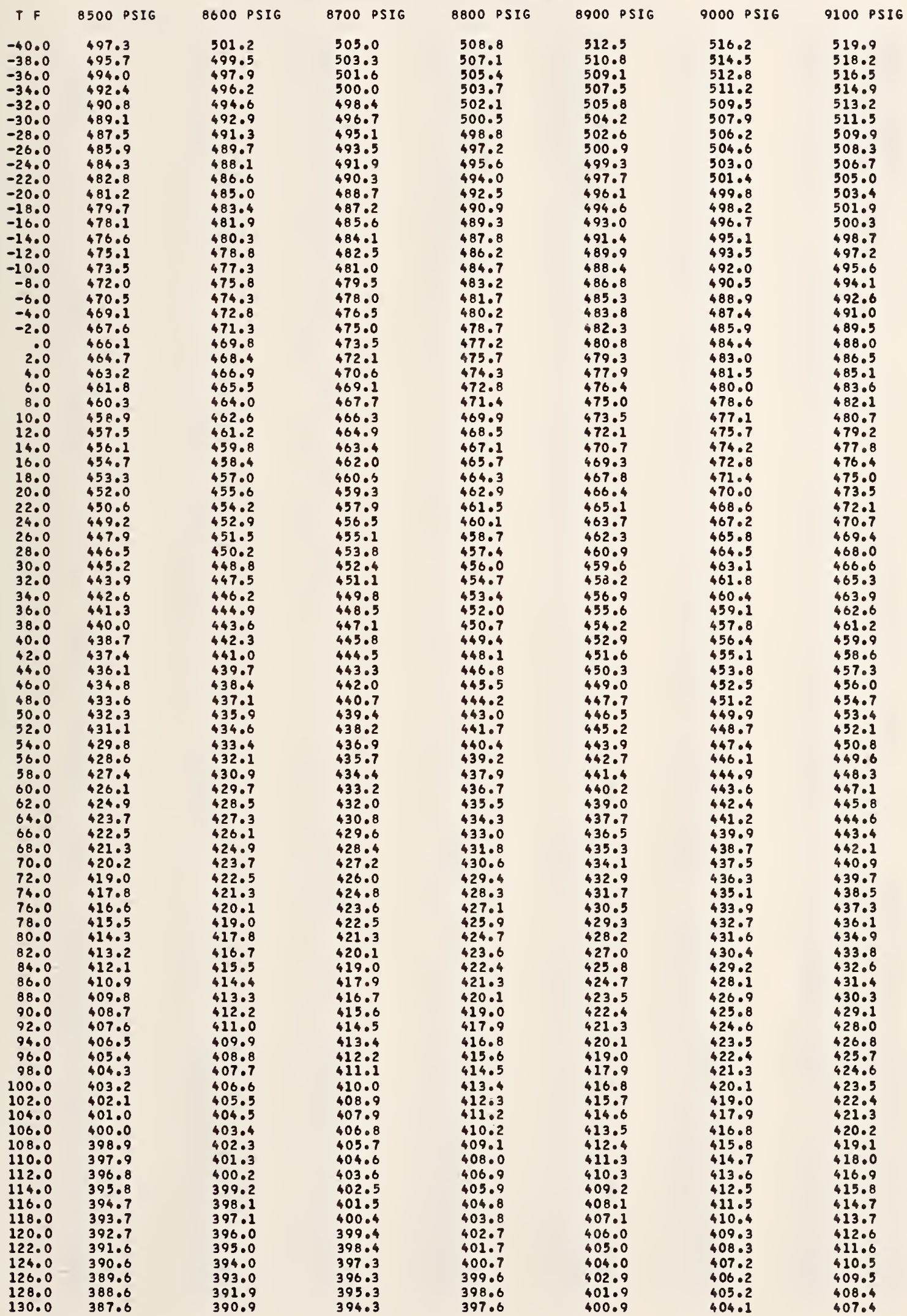


HYDRDGEN CONTENTS TABLE (SCF/CU FT).

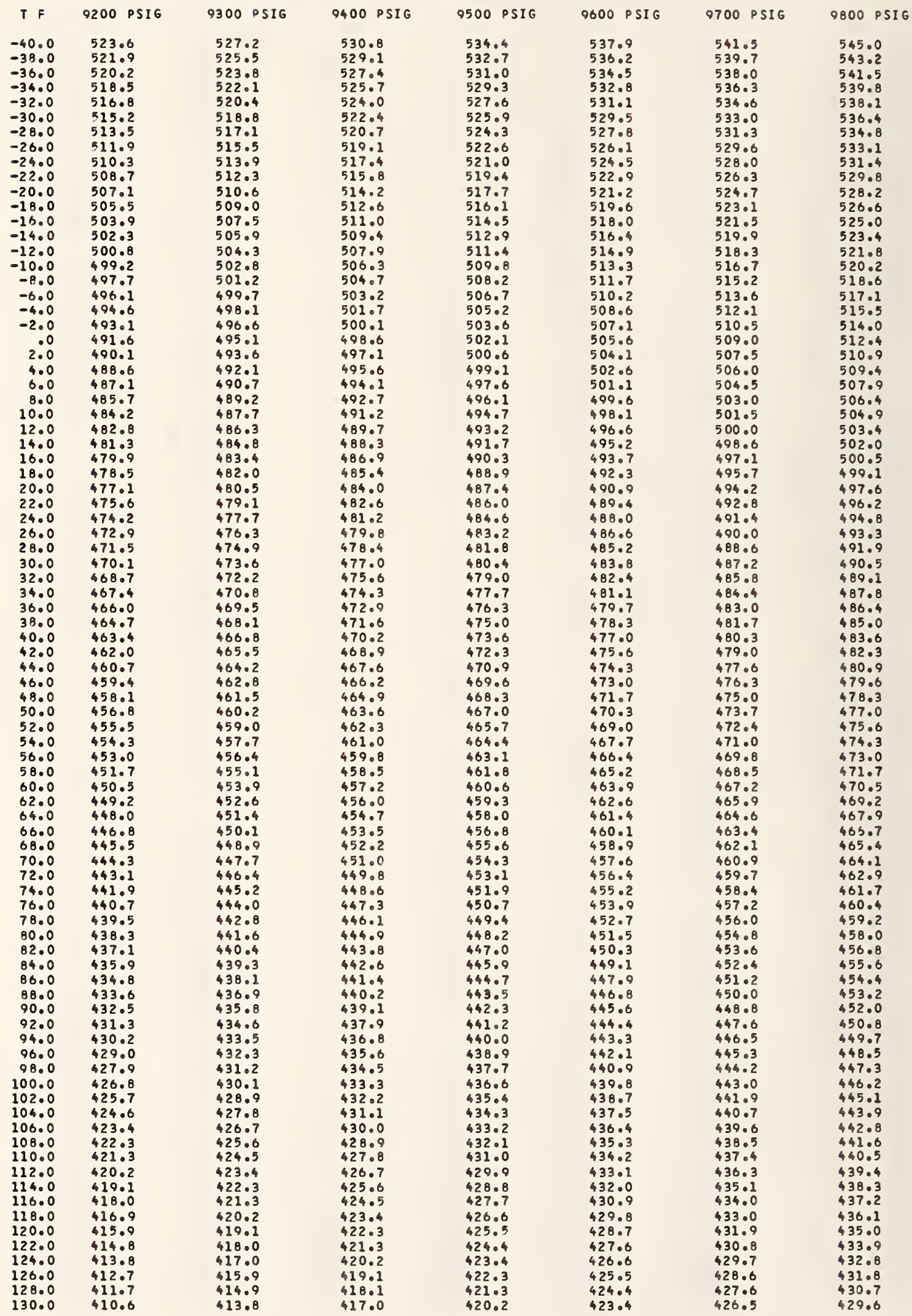


HYDROGEN CONTENTS TABLE (SCF/CU FT).

TF 9900 PSIG 10000 PSIG

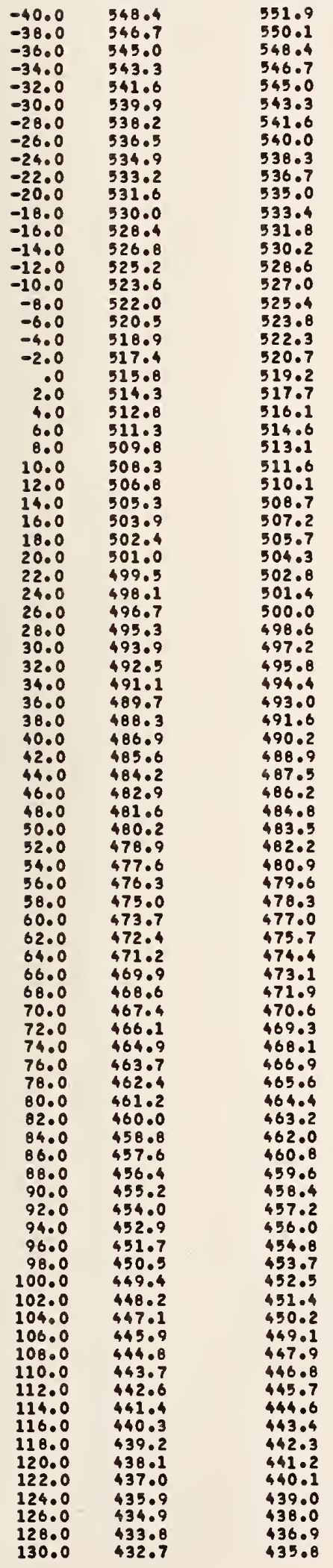


9.0

Density Tables in SI Units 
9.1

Oxygen Density $\left(\mathrm{kg} / \mathrm{m}^{3}\right)$ 
QXYGEN OENSITY (KG/MB).

\begin{tabular}{|c|c|c|c|c|c|c|c|}
\hline$T K$ & .5 MPA & $1.0 \mathrm{MPA}$ & $1.5 \mathrm{MPA}$ & $2.0 \mathrm{MPA}$ & $2.5 \mathrm{MPA}$ & $3.0 \mathrm{MPA}$ & 3.5 \\
\hline $\begin{array}{l}200.0 \\
202.0\end{array}$ & $\begin{array}{l}9.770 \\
9.668\end{array}$ & $\begin{array}{l}19.85 \\
19.63\end{array}$ & $\begin{array}{l}30.25 \\
29.91\end{array}$ & $\begin{array}{l}41.01 \\
40.51\end{array}$ & $\begin{array}{l}52.14 \\
51.47\end{array}$ & $\begin{array}{l}63.66 \\
62.80\end{array}$ & $\begin{array}{l}75.60 \\
74.53\end{array}$ \\
\hline $\begin{array}{l}204.0 \\
206.0\end{array}$ & $\begin{array}{l}9.569 \\
9.471\end{array}$ & $\begin{array}{l}19.42 \\
19.21\end{array}$ & $\begin{array}{l}29.57 \\
29.24\end{array}$ & $\begin{array}{l}40.03 \\
39.56\end{array}$ & $\begin{array}{l}50.83 \\
50.23\end{array}$ & $\begin{array}{l}61.98 \\
61.18\end{array}$ & $\begin{array}{r}73.49 \\
72.49\end{array}$ \\
\hline 208.0 & 9.376 & 19.01 & 28.92 & 39.11 & 49.60 & 60.40 & 71.53 \\
\hline 210.0 & 9.283 & 18.81 & 28.60 & 38.66 & 49.01 & 59.65 & 70.59 \\
\hline 212.0 & 9.192 & 18.62 & 28.29 & 38.23 & 48.43 & 58.92 & 69.69 \\
\hline 214.0 & 9.102 & 18.43 & 27.99 & 37.81 & 47.87 & 58.21 & 68.81 \\
\hline 215.0 & 9.014 & 18.25 & 27.70 & 37.39 & 47.33 & 57.52 & 67.96 \\
\hline 218.0 & 8.928 & 18.06 & 27.42 & 36.99 & 46.80 & 56.85 & 67.14 \\
\hline 220.0 & 8.844 & 17.89 & 27.14 & 36.60 & 46.28 & 56.19 & 66.34 \\
\hline 222.0 & 8.761 & 17.71 & 26.86 & 36.22 & 45.78 & 55.56 & 35.56 \\
\hline 224.0 & 8.680 & 17.54 & 26.59 & 35.84 & 45.29 & 54.94 & 64.80 \\
\hline 226.0 & 8.601 & 17.38 & 26.33 & 35.47 & 44.81 & 54.34 & 64.06 \\
\hline 228.0 & 8.522 & 17.21 & 26.08 & 35.12 & 44.34 & 53.75 & 63.34 \\
\hline $\begin{array}{l}230.0 \\
232.0\end{array}$ & $\begin{array}{l}8.446 \\
8.371\end{array}$ & $\begin{array}{l}17.05 \\
16.90\end{array}$ & $\begin{array}{l}25.83 \\
25.58\end{array}$ & $\begin{array}{l}34.77 \\
34.42\end{array}$ & 43.88 & 53.17 & 62.65 \\
\hline 234.0 & 8.297 & 16.74 & 25.34 & 34.09 & 43.00 & $\begin{array}{l}52.62 \\
52.07\end{array}$ & $\begin{array}{l}61.96 \\
61.30\end{array}$ \\
\hline 236.0 & 8.224 & 16.59 & 25.10 & 33.76 & 42.57 & 51.54 & 60.65 \\
\hline $\begin{array}{l}238.0 \\
240.0\end{array}$ & $\begin{array}{l}8.153 \\
8.083\end{array}$ & $\begin{array}{l}16.44 \\
16.30\end{array}$ & $\begin{array}{l}24.87 \\
24.64\end{array}$ & $\begin{array}{l}33.44 \\
33.13\end{array}$ & $\begin{array}{l}42.16 \\
41.75\end{array}$ & $\begin{array}{l}51.02 \\
50.51\end{array}$ & $\begin{array}{l}60.02 \\
59.40\end{array}$ \\
\hline 242.0 & 8.014 & 16.15 & 24.42 & 32.82 & 41.35 & 50.01 & 58.80 \\
\hline 244.0 & 7.946 & 16.01 & 24.20 & 32.52 & 40.96 & 49.52 & 58.21 \\
\hline 245.0 & 7.880 & 15.88 & 23.99 & 32.22 & 40.57 & 49.05 & 57.64 \\
\hline 248.0 & 7.815 & 15.74 & 23.78 & 31.93 & 40.20 & 48.58 & 57.08 \\
\hline $\begin{array}{l}250.0 \\
252.0\end{array}$ & $\begin{array}{l}7.750 \\
7.687\end{array}$ & 15.61 & 23.57 & 31.65 & 39.83 & 48.12 & 56.53 \\
\hline $\begin{array}{l}232.0 \\
254.0\end{array}$ & $\begin{array}{l}7.687 \\
7.625\end{array}$ & 15.48 & 23.37 & 31.37 & 39.47 & 47.68 & 55.99 \\
\hline 256.0 & 7.564 & $\begin{array}{l}15.35 \\
15.22\end{array}$ & $\begin{array}{l}23.17 \\
22.98\end{array}$ & $\begin{array}{l}31.09 \\
30.83\end{array}$ & $\begin{array}{l}39.12 \\
38.77\end{array}$ & $\begin{array}{l}47.24 \\
46.81\end{array}$ & $\begin{array}{l}55.46 \\
54.95\end{array}$ \\
\hline 258.0 & 7.504 & 15.10 & 22.78 & 30.56 & 38.43 & 46.39 & 54.44 \\
\hline 260.0 & 7.445 & 14.98 & 22.60 & 30.30 & 39.10 & 45.98 & 53.95 \\
\hline 262.0 & 7.387 & 14.86 & 22.41 & 30.05 & 37.77 & 45.58 & 53.47 \\
\hline 264.0 & 7.329 & 14.74 & 22.23 & 29.80 & 37.45 & 45.18 & 52.99 \\
\hline 266.0 & 7.273 & 14.62 & 22.05 & 29.55 & 37.14 & 44.79 & 52.53 \\
\hline 268.0 & 7.217 & 14.51 & 21.87 & 29.31 & 36.83 & 44.41 & 52.07 \\
\hline 270.0 & 7.163 & 14.40 & 21.70 & 29.08 & 36.52 & 44.04 & 51.62 \\
\hline 272.0 & 7.109 & 14.29 & 21.53 & 28.84 & 36.23 & 43.67 & 51.18 \\
\hline 274.0 & 7.056 & 14.18 & 21.36 & 28.62 & 35.93 & 43.31 & 50.75 \\
\hline 276.0 & 7.004 & 14.07 & 21.20 & 28.39 & 35.54 & 42.96 & 50.33 \\
\hline 278.0 & 6.952 & 13.97 & 21.04 & 28.17 & 35.36 & 42.61 & 49.92 \\
\hline 280.0 & 6.902 & 13.86 & 20.88 & 27.95 & 35.08 & 42.27 & 49.51 \\
\hline 282.0 & 6.852 & 13.76 & 20.72 & 27.74 & 34.81 & 41.93 & 49.11 \\
\hline 284.0 & 6.803 & 13.06 & 20.57 & 27.53 & 34.54 & 41.60 & 48.72 \\
\hline 286.0 & 6.754 & 13.56 & 20.42 & 27.32 & 34.28 & 41.28 & 48.33 \\
\hline 288.0 & 6.706 & 13.46 & 20.27 & 27.12 & 34.02 & 40.96 & 47.95 \\
\hline $\begin{array}{l}290.0 \\
292.0\end{array}$ & 6.659 & $\begin{array}{l}13.37 \\
13.27\end{array}$ & $\begin{array}{l}20.12 \\
19.97\end{array}$ & 26.92 & 33.76 & 40.65 & 47.58 \\
\hline 294.0 & $\begin{array}{l}6.613 \\
6.567\end{array}$ & $\begin{array}{l}13.27 \\
13.18\end{array}$ & $\begin{array}{l}19.97 \\
19.83\end{array}$ & $\begin{array}{l}26.72 \\
26.53\end{array}$ & $\begin{array}{l}33.51 \\
33.26\end{array}$ & $\begin{array}{l}40.34 \\
40.04\end{array}$ & $\begin{array}{l}47.21 \\
46.85\end{array}$ \\
\hline 295.0 & 6.522 & 13.09 & 19.69 & 26.33 & 33.02 & 39.74 & 46.50 \\
\hline 298.0 & 6.478 & 12.99 & 19.55 & 26.15 & 32.78 & 39.45 & 46.15 \\
\hline 300.0 & 0.434 & 12.91 & 19.41 & 25.96 & 32.54 & 39.16 & 45.81 \\
\hline 302.0 & 6.390 & 12.82 & 19.28 & 25.78 & 32.31 & 38.87 & 45.47 \\
\hline 304.0 & 6.348 & 12.73 & 19.15 & 25.60 & 32.08 & 38.59 & 45.14 \\
\hline 306.0 & 6.306 & 12.64 & 19.02 & 25.42 & 31.85 & 38.32 & 44.81 \\
\hline $\begin{array}{l}308.0 \\
310.0\end{array}$ & $\begin{array}{l}0.264 \\
6.223\end{array}$ & $\begin{array}{l}12.56 \\
12.48\end{array}$ & $\begin{array}{l}18.89 \\
18.76\end{array}$ & $\begin{array}{l}25.24 \\
25.07\end{array}$ & $\begin{array}{l}31.53 \\
31.41\end{array}$ & 39.05 & 44.49 \\
\hline 312.0 & 6.182 & 12.39 & 18.63 & 24.90 & $\begin{array}{l}31.41 \\
31.25\end{array}$ & $\begin{array}{l}37.78 \\
37.52\end{array}$ & $\begin{array}{l}44.17 \\
43.86\end{array}$ \\
\hline 314.0 & 0.143 & 12.31 & 18.51 & 24.73 & 30.98 & 37.26 & 43.55 \\
\hline 316.0 & 6.103 & 12.23 & 18.39 & 24.57 & 30.77 & 37.00 & 43.25 \\
\hline 318.0 & 0.064 & 12.15 & 18.27 & 24.41 & 30.57 & 36.75 & 42.95 \\
\hline 320.0 & 6.026 & 12.08 & 18.15 & 24.24 & 30.36 & 36.50 & 42.66 \\
\hline 322.0 & 5.988 & 12.00 & 18.03 & 24.09 & 30.15 & 36.26 & 42.37 \\
\hline 324.0 & 5.950 & 11.92 & 17.92 & 23.93 & 29.96 & 35.02 & 42.09 \\
\hline 326.0 & 5.913 & 11.85 & 17.80 & 23.78 & 29.77 & 35.78 & 41.81 \\
\hline 328.0 & 5.877 & 11.77 & 17.69 & 23.62 & 29.58 & 35.54 & 41.53 \\
\hline 330.0 & 5.841 & 11.70 & 17.58 & 23.47 & 29.38 & 35.31 & 41.26 \\
\hline 332.0 & 5.805 & 11.63 & 17.47 & 23.33 & 29.20 & 35.08 & 40.99 \\
\hline 334.0 & 5.770 & 11.56 & 17.36 & 23.18 & 29.01 & 34.86 & 40.72 \\
\hline 336.0 & 5.735 & 11.49 & 17.25 & 23.03 & 28.83 & 36.64 & 40.46 \\
\hline 338.0 & 3.701 & 11.42 & 17.15 & 22.89 & 28.65 & 34.42 & 40.20 \\
\hline 340.0 & & 11.35 & 17.04 & 22.75 & 28.47 & 34.20 & 39.95 \\
\hline 342.0 & 5.634 & 11.28 & 15.94 & 22.61 & 28.30 & 33.99 & 39.69 \\
\hline 344.0 & 5.601 & 11.21 & 16.84 & 22.48 & 29.12 & 33.78 & 39.45 \\
\hline 345.0 & 5.568 & 11.15 & 16.74 & 22.34 & 27.95 & 33.57 & 39.20 \\
\hline 348.0 & 5.535 & 11.08 & 16.64 & 22.21 & 27.78 & 33.37 & 38.96 \\
\hline 350.0 & 5.504 & 11.02 & 16.54 & 22.07 & 27.52 & 33.17 & 38.72 \\
\hline 352.0 & 5.472 & 10.95 & 16.44 & 21.94 & 27.45 & 32.97 & 38.49 \\
\hline 354.0 & 5.441 & 10.89 & 16.35 & 21.81 & 27.29 & 32.77 & 38.26 \\
\hline 356.0 & 5.410 & 10.83 & 16.25 & 21.69 & 27.13 & 32.57 & 38.03 \\
\hline 358.0 & 5.379 & 10.77 & 16.16 & 21.56 & 26.97 & 32.38 & 37.80 \\
\hline 360.0 & 5.349 & 10.71 & 16.07 & 21.44 & 26.81 & 32.19 & 37.58 \\
\hline 362.0 & 5.319 & 10.65 & 15.98 & 21.31 & 26.66 & 32.00 & 37.36 \\
\hline 364.0 & 5.290 & 10.59 & 15.89 & 21.19 & 25.53 & 31.82 & 37.14 \\
\hline 360.0 & 5.261 & 10.53 & 15.80 & 21.07 & 26.35 & 31.64 & 36.92 \\
\hline 368.0 & 5.232 & 10.47 & 15.71 & 20.96 & 26.20 & 31.46 & 36.71 \\
\hline 370.0 & 5.203 & 10.41 & 15.62 & 20.84 & 26.06 & 31.28 & 36.50 \\
\hline
\end{tabular}


OXYGEN OENSITY (KG/M3).

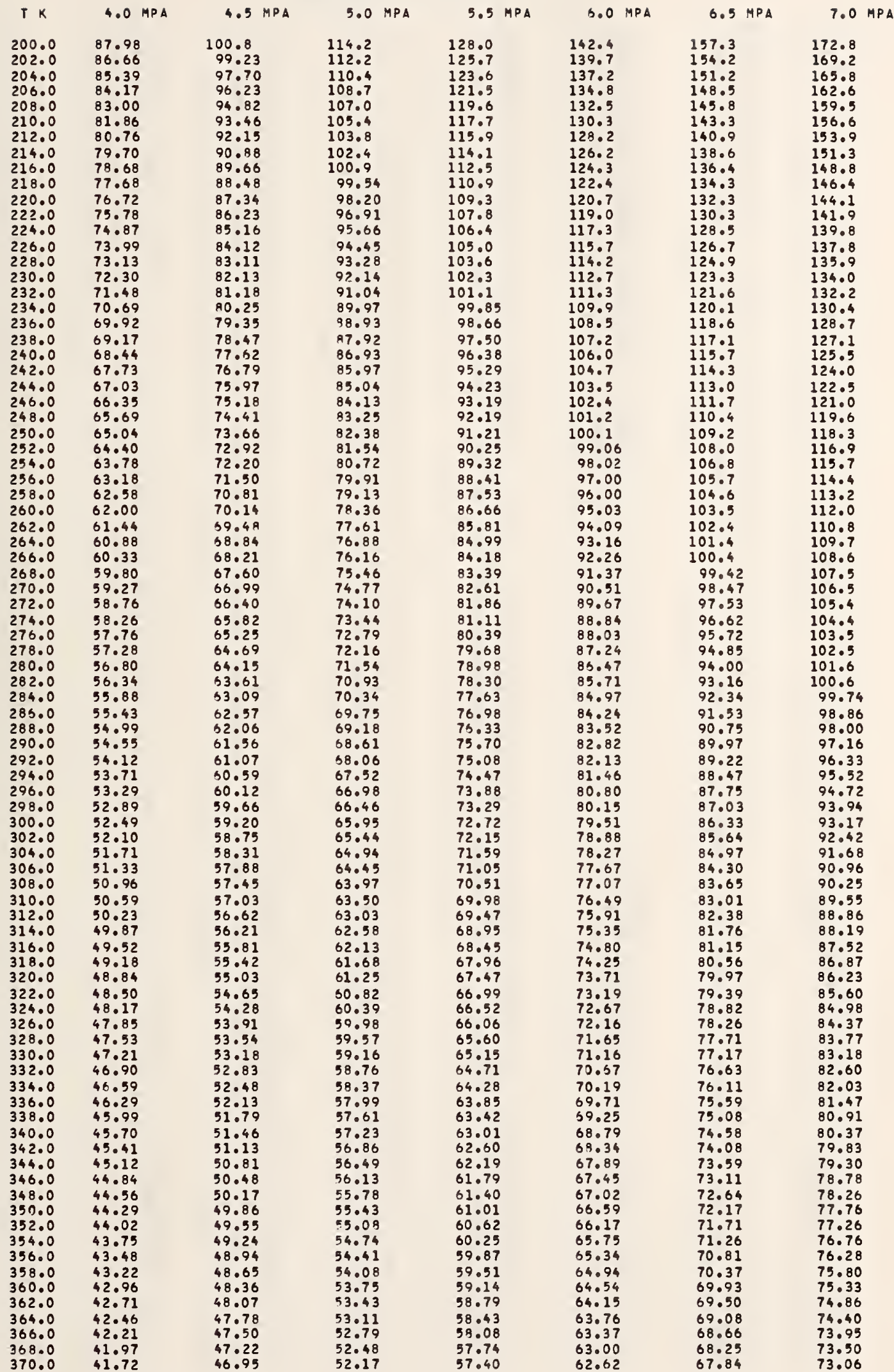


OXYGEN DENSITY (KG/M3).

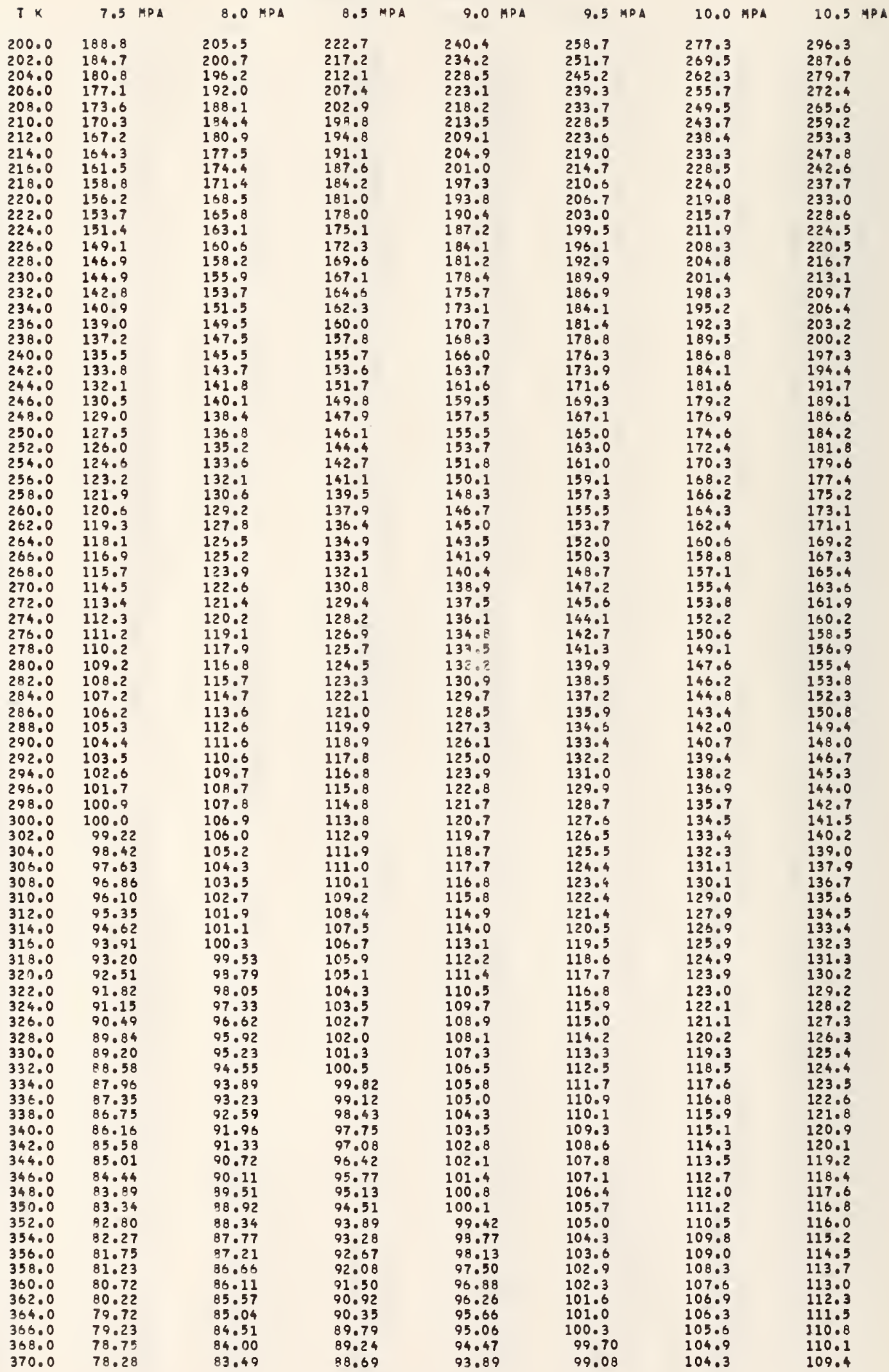


OXYGEN DENSITY (KG/H3).

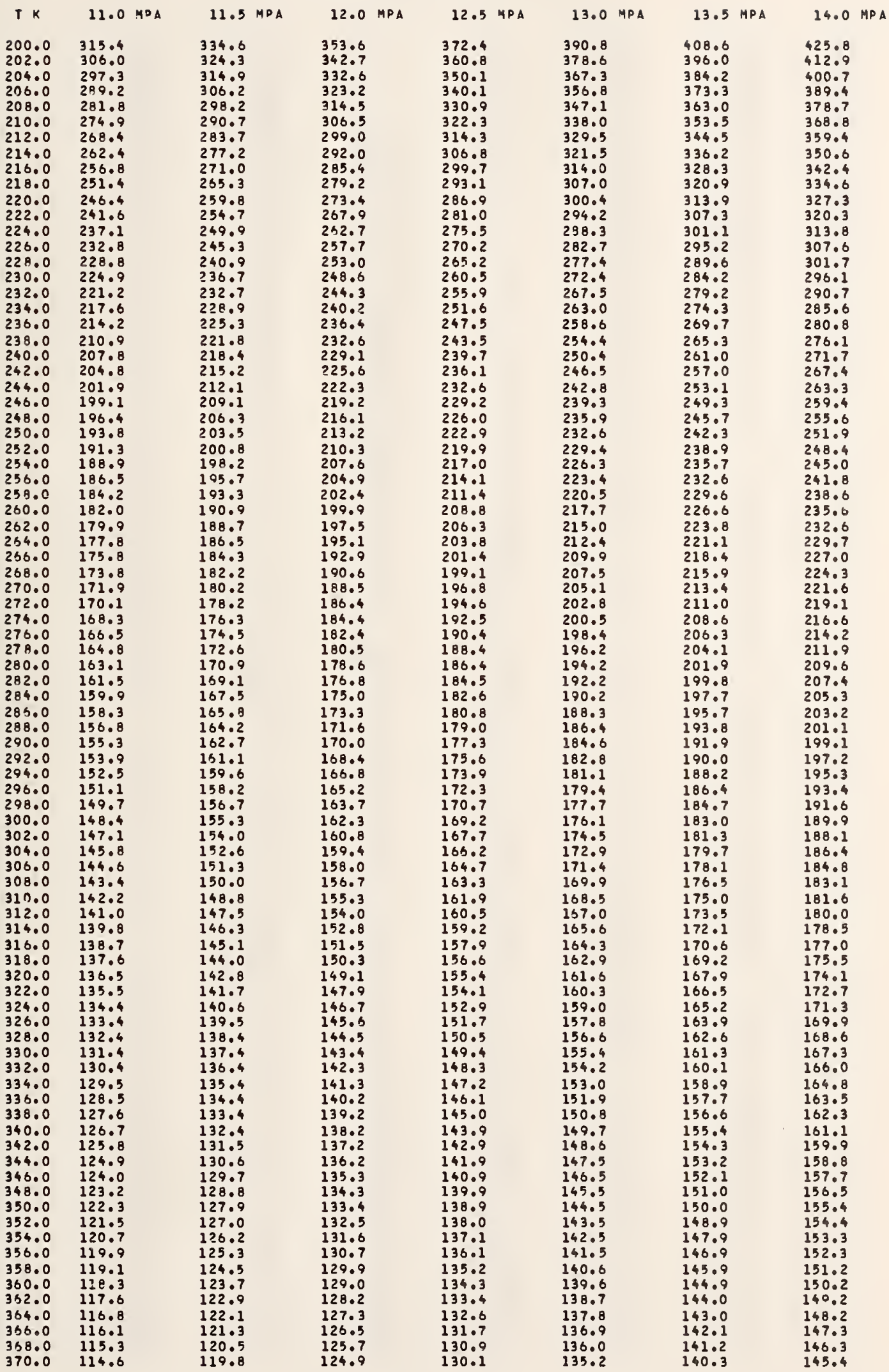


OXYGEN OENSITY (KG/M3).

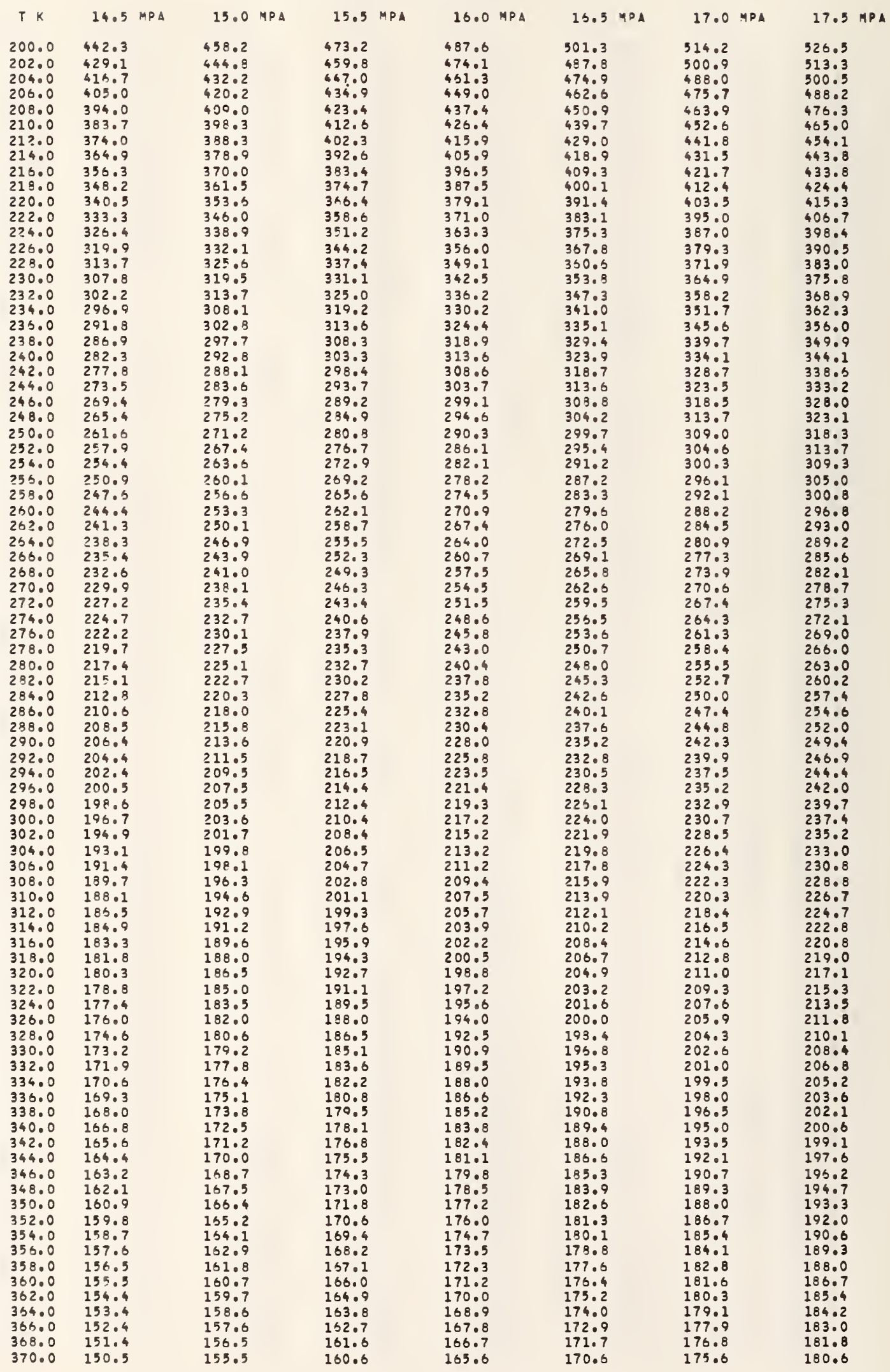


DXYGEN OENSITY (KG/MB).

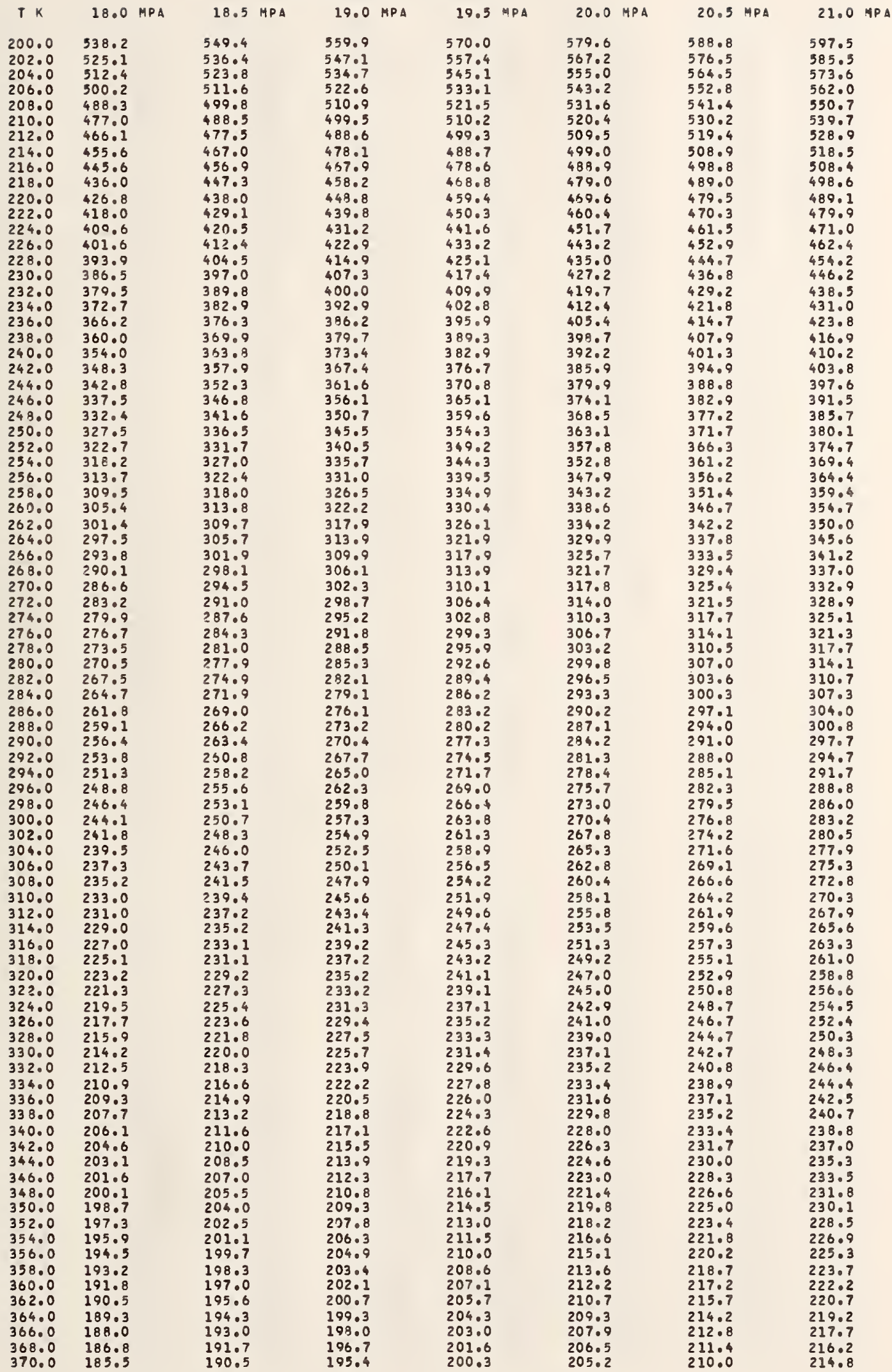


OXYGEN DENSITY (KG/M3)

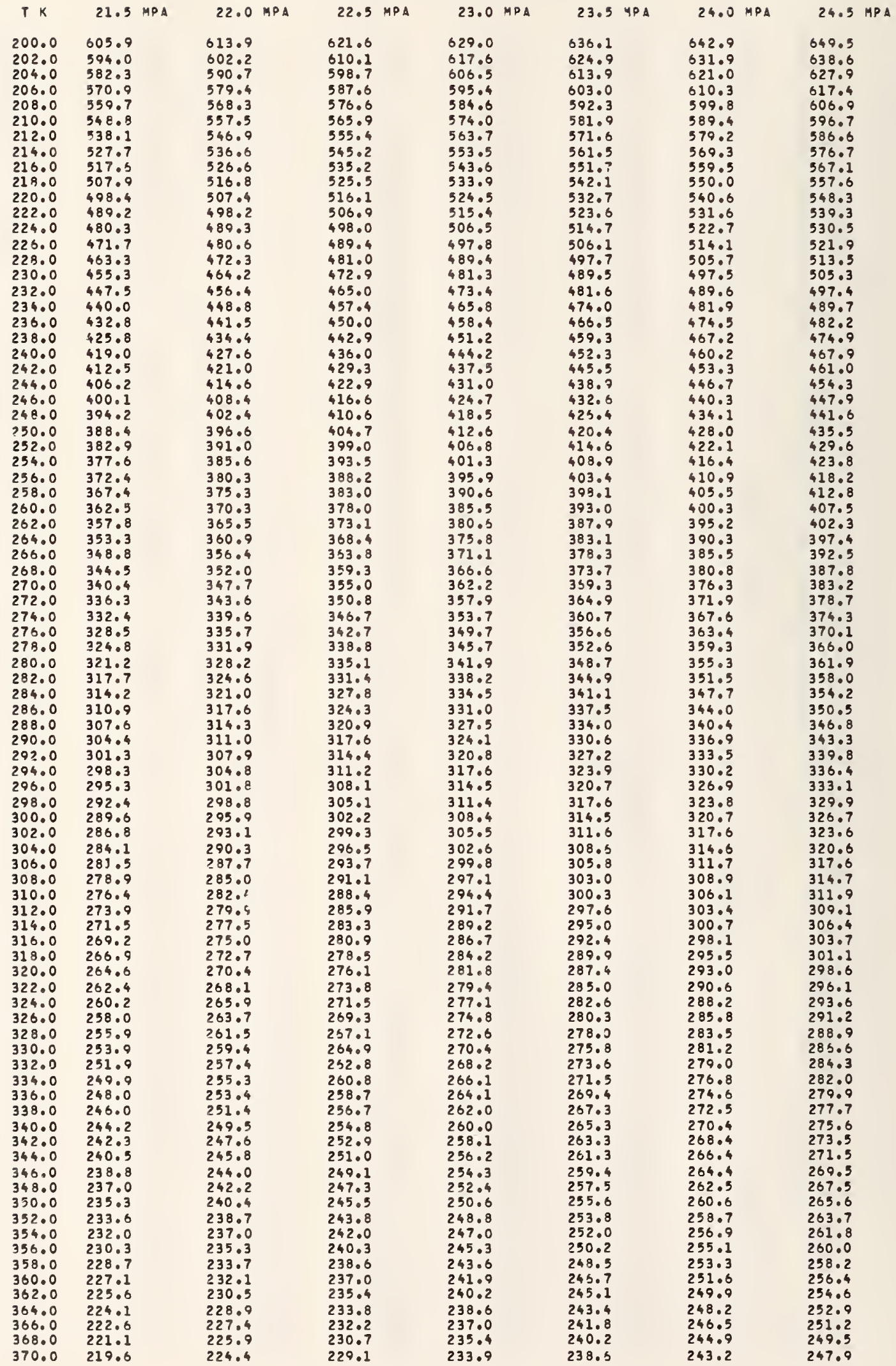


OXYGEN OENSITY (KG/M3).

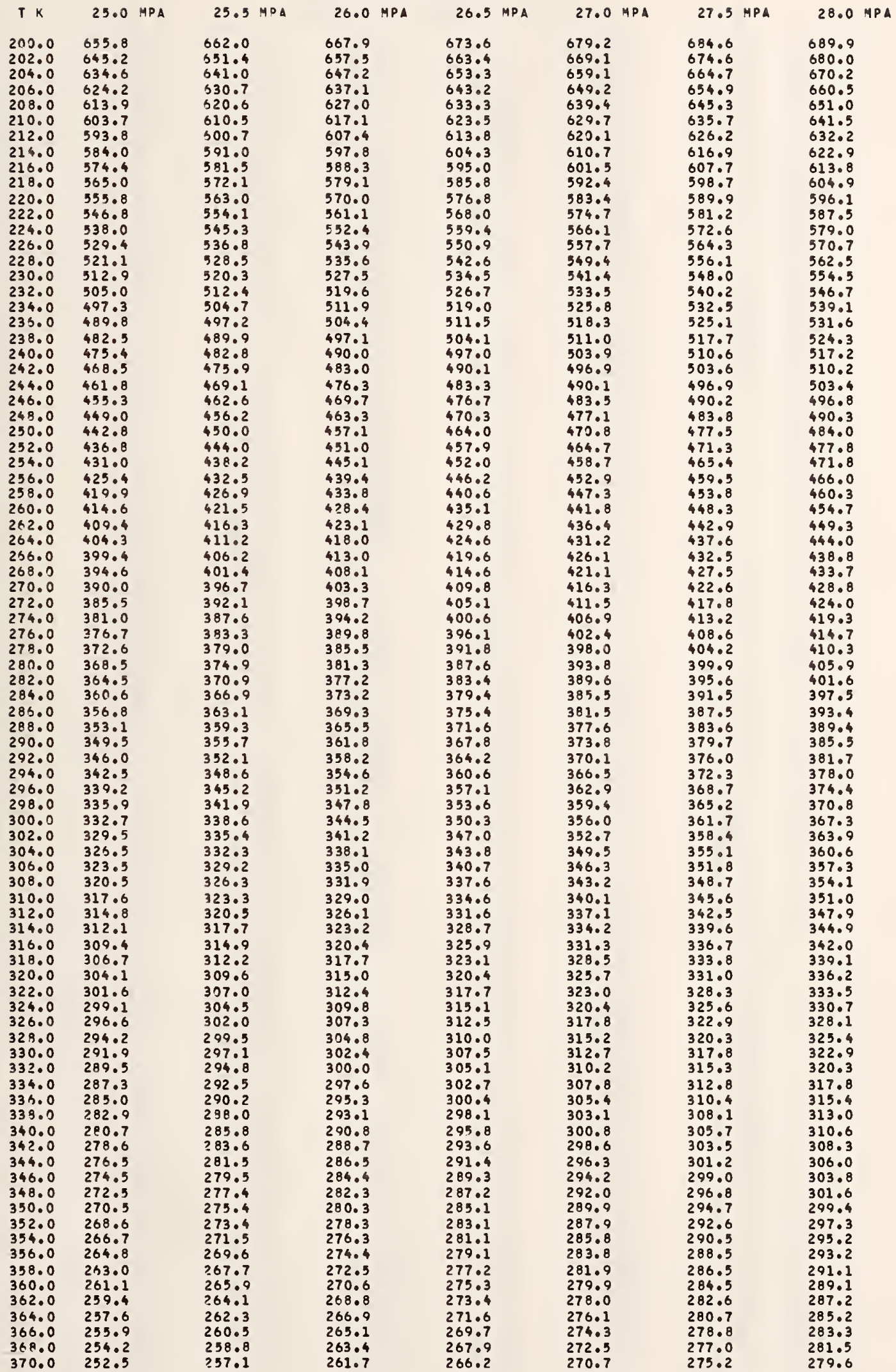


OXYGEN OENSITY (KG/M3).

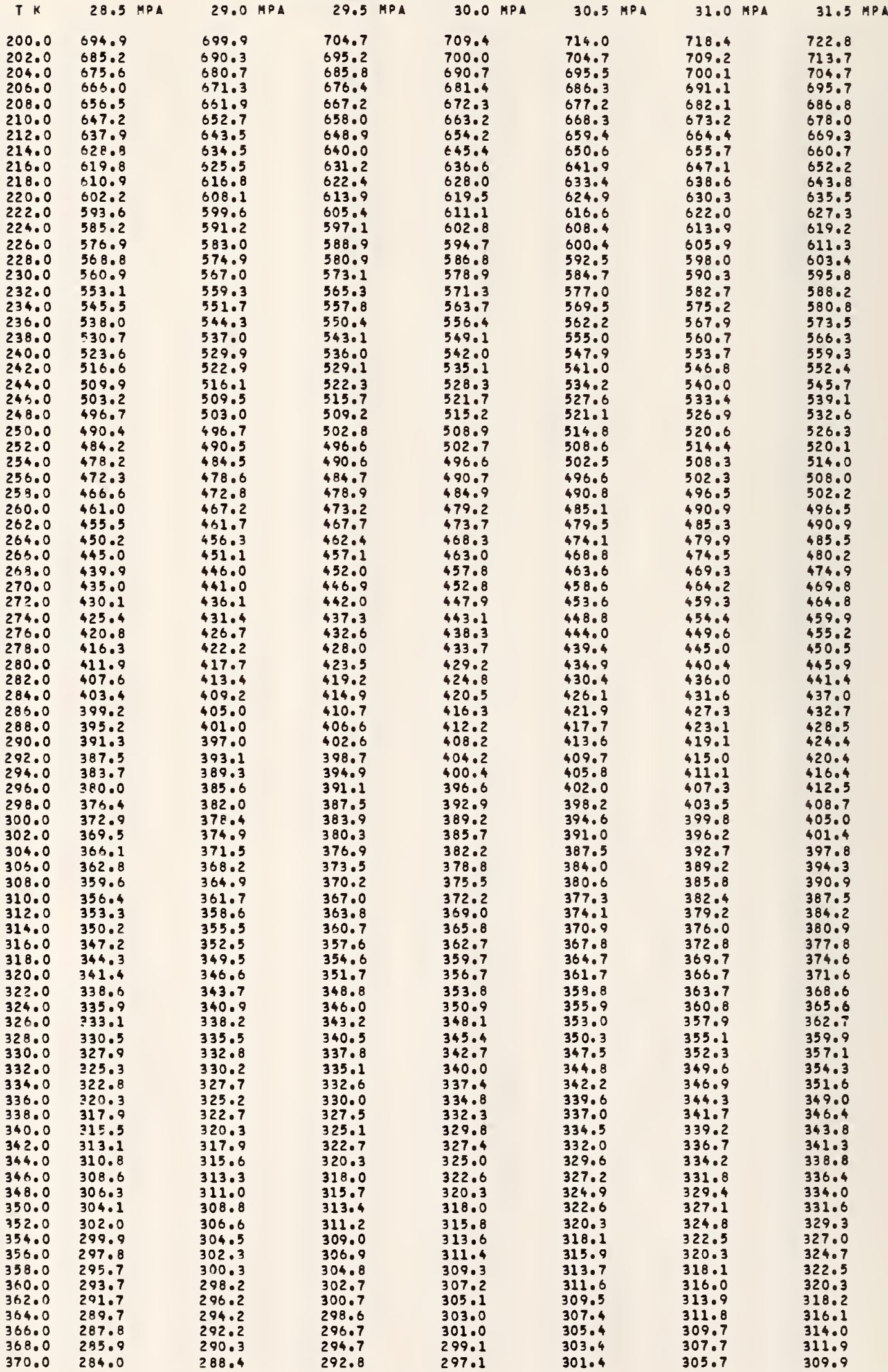


OXYGEN DENSITY (KG/43).

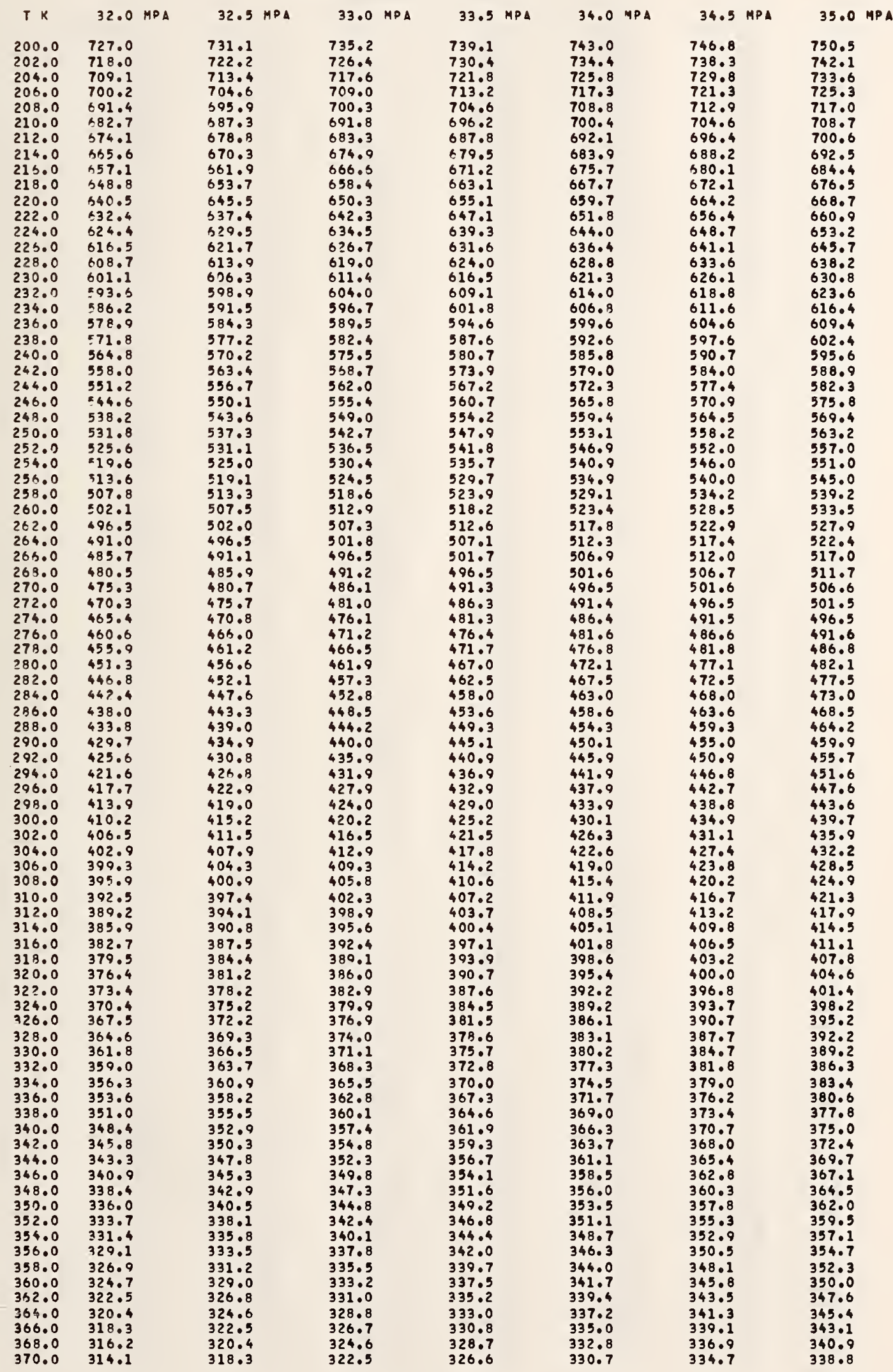


DXYGEN DENSITY (KEIMB)

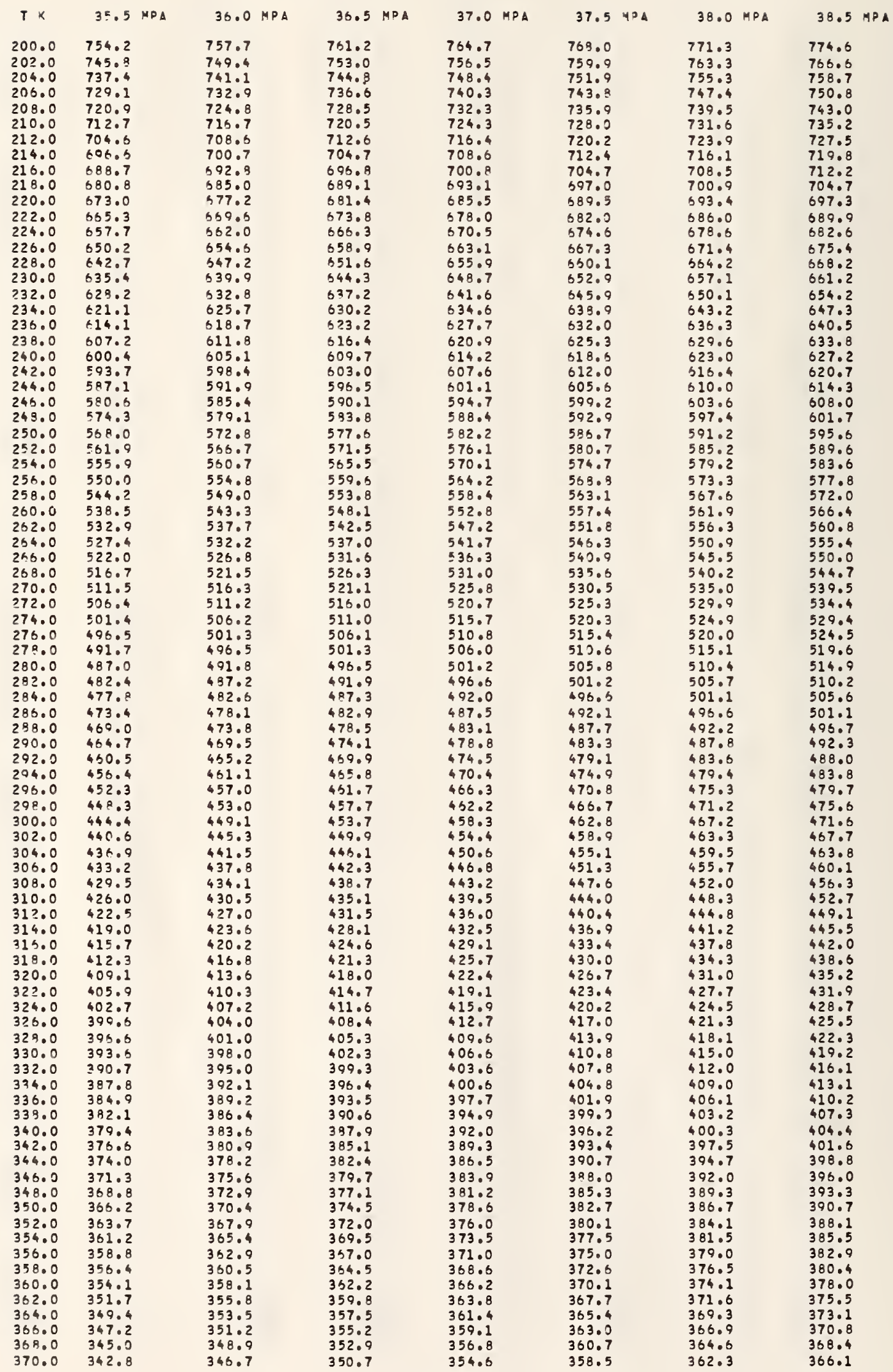


DXYGEN DENSITY (KG/N3).

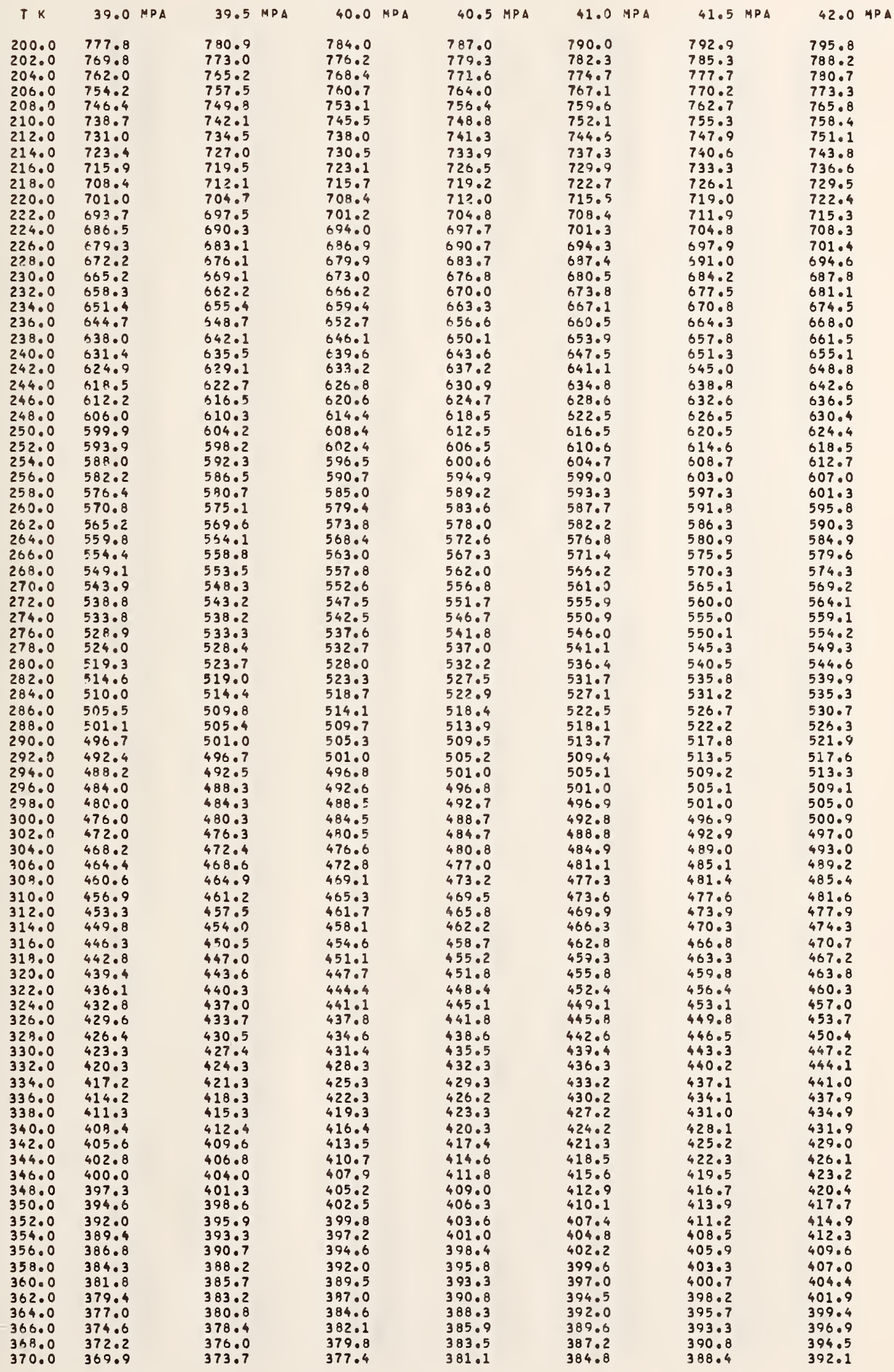


OXYGEN DENSITY (KG/M3).

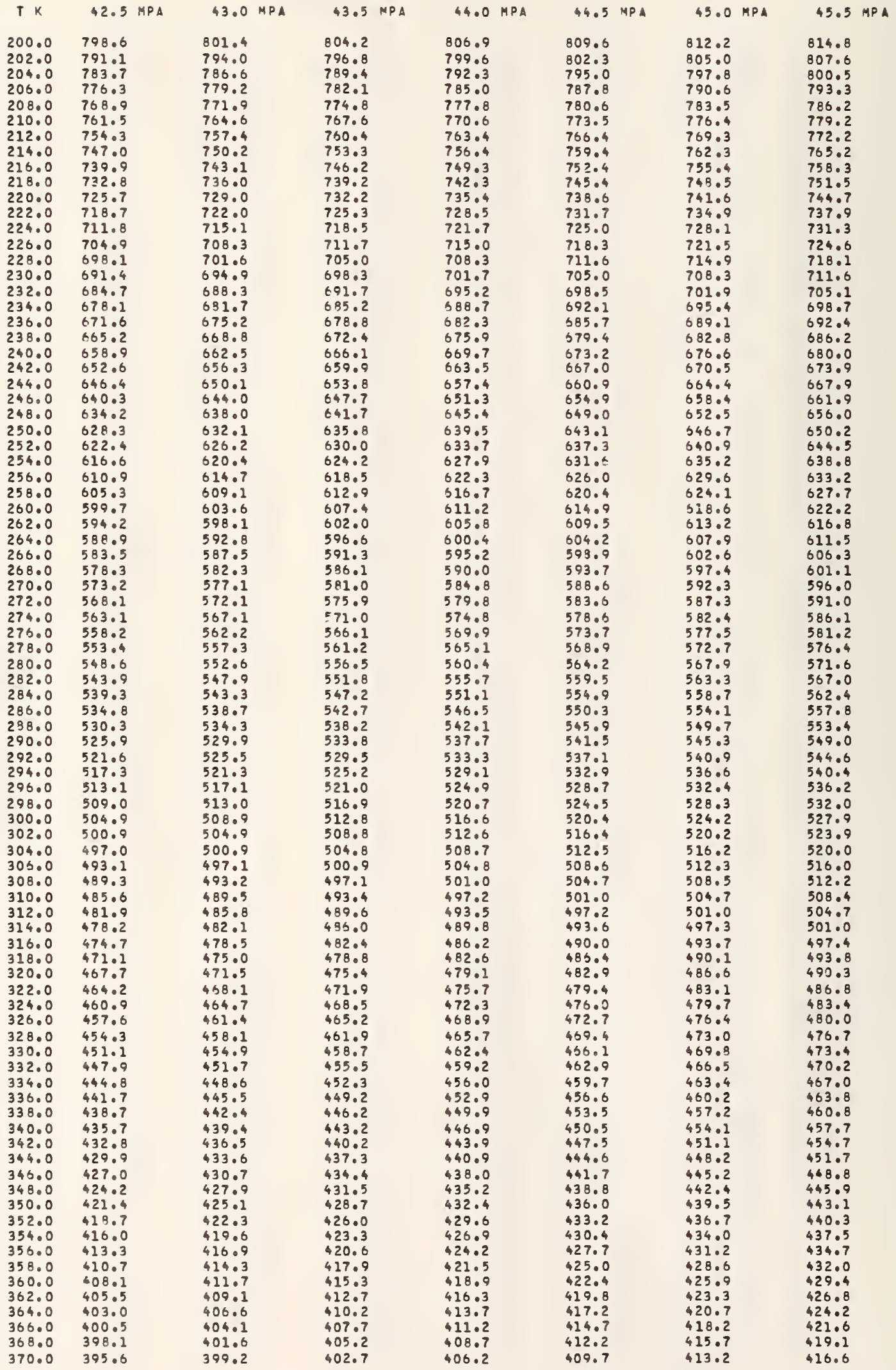


OXYGEN DENSITY (KG/M3).

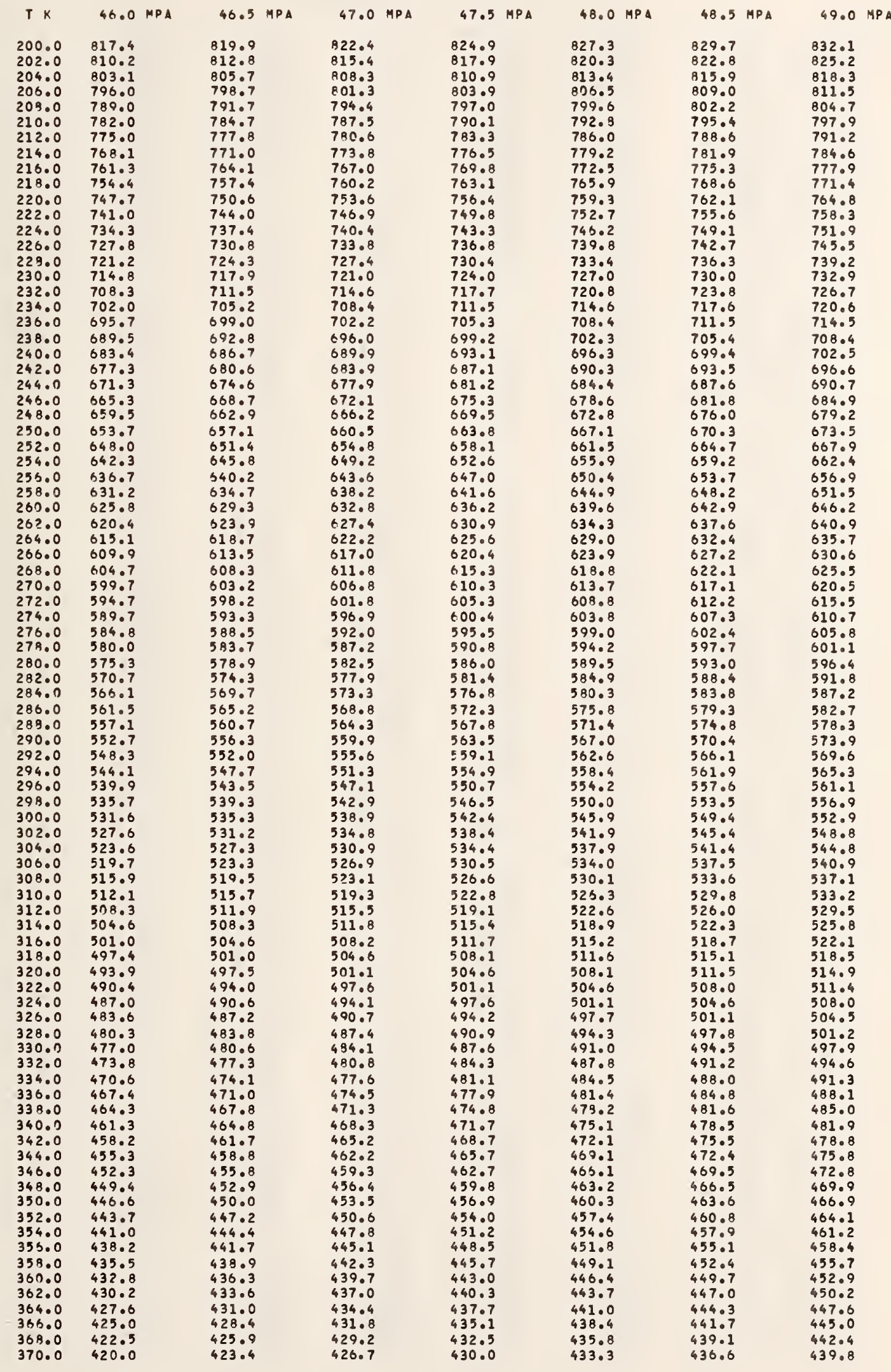


OXYGEN DENSITY (KG/H3).

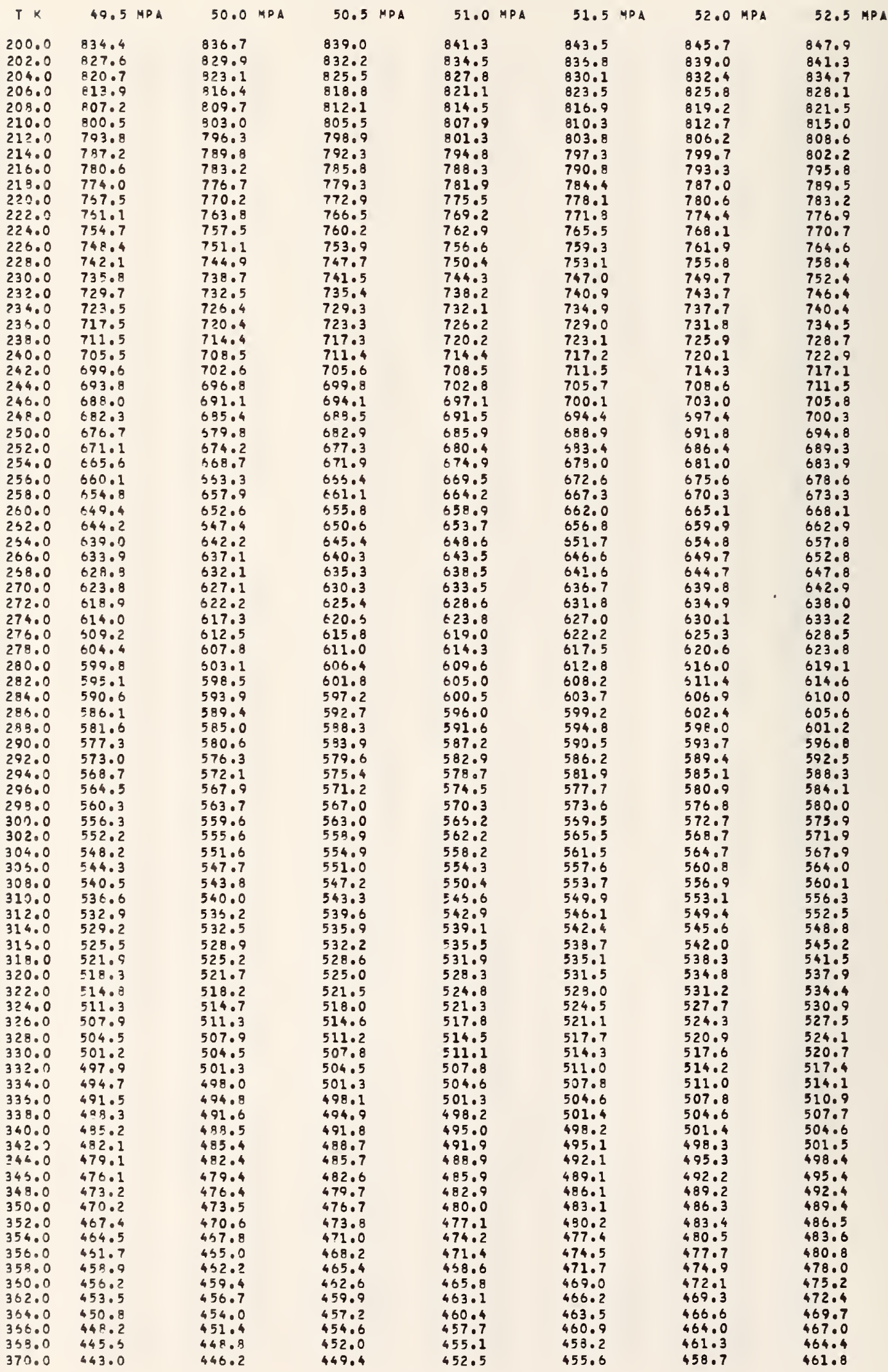


OXYGEN DENSITY (KEIM3).

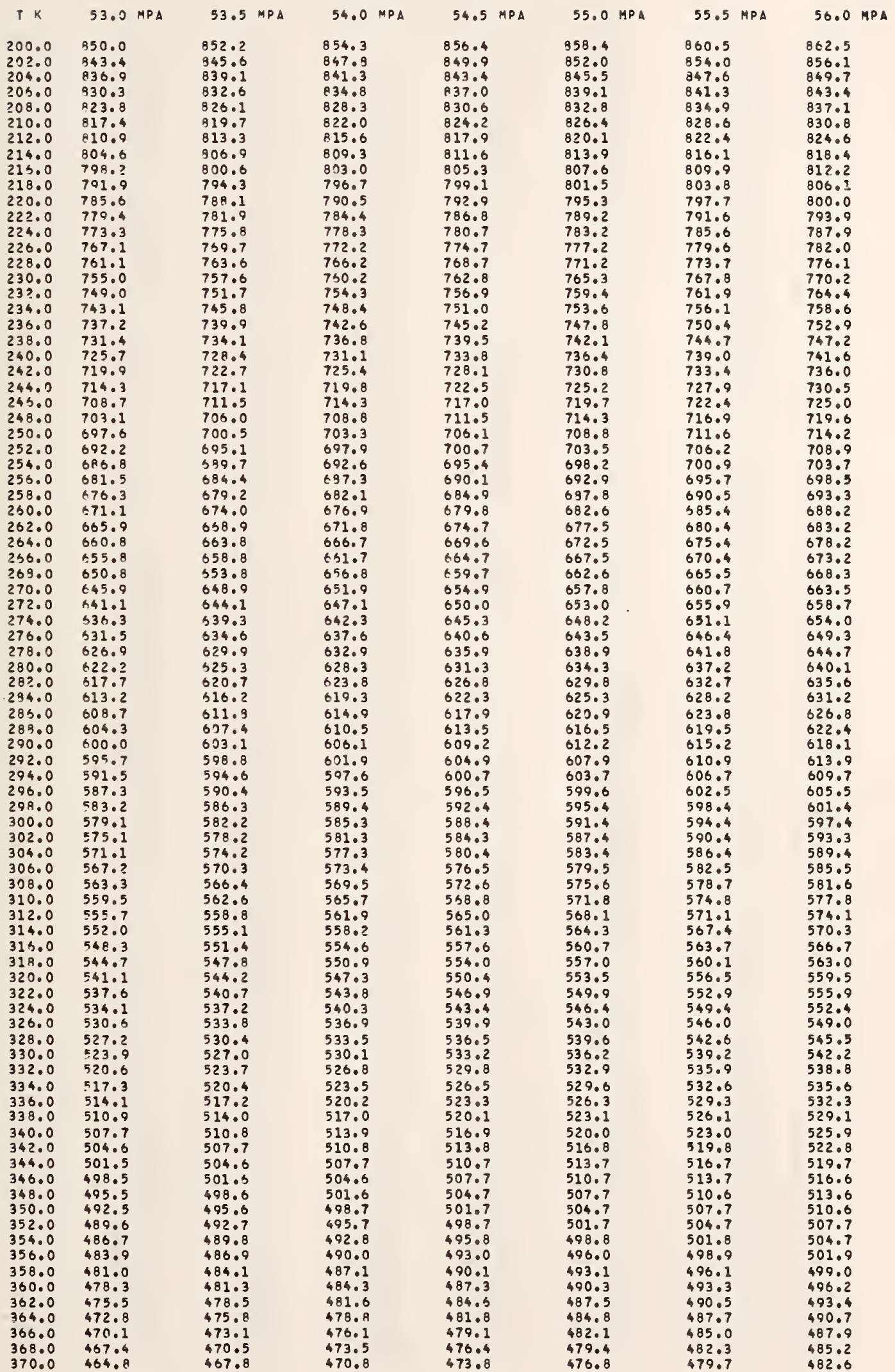


OXYGEN DENSITY (KG/H3).

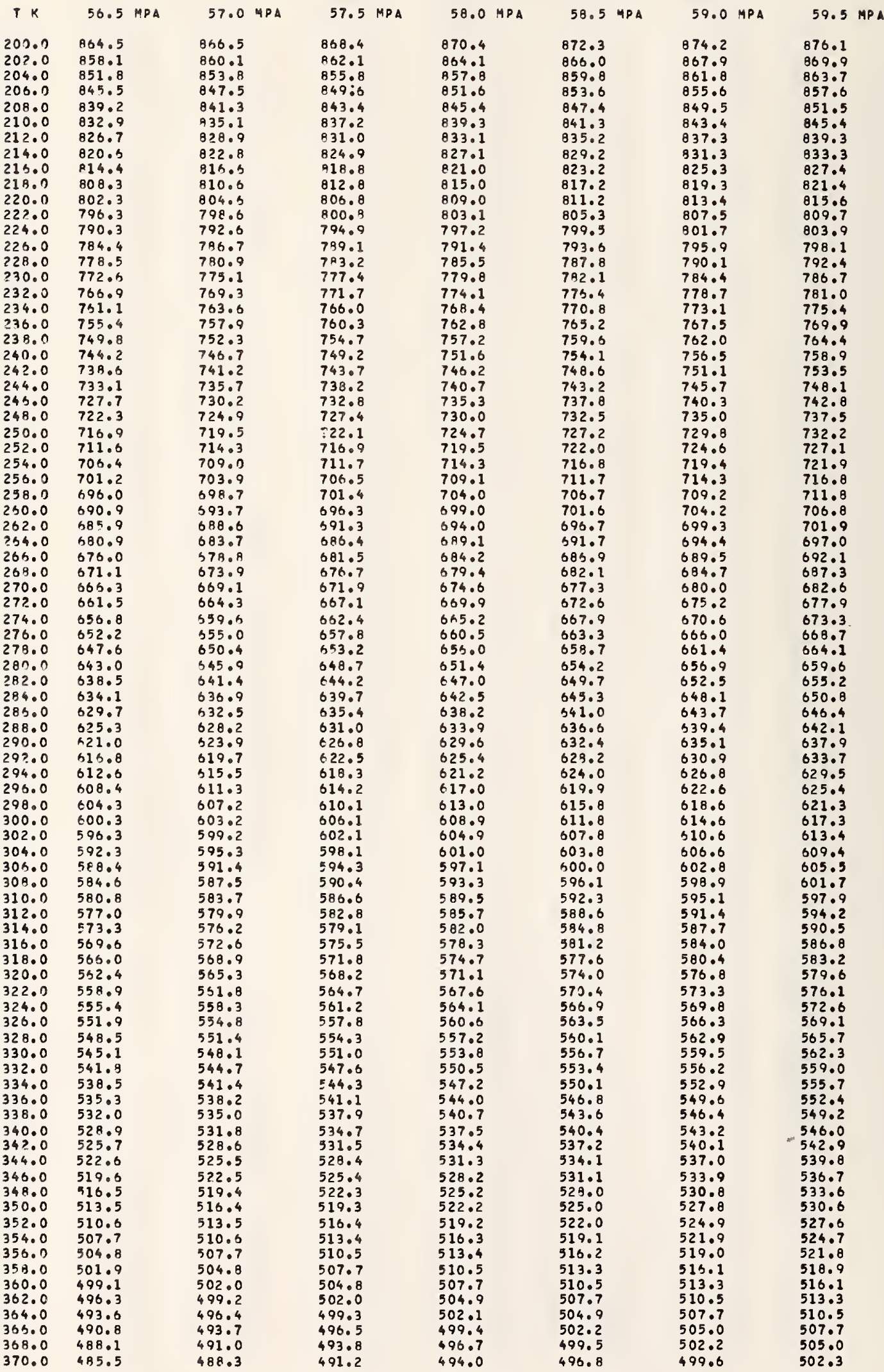


OXYGEN DENSITY (KG/M3).

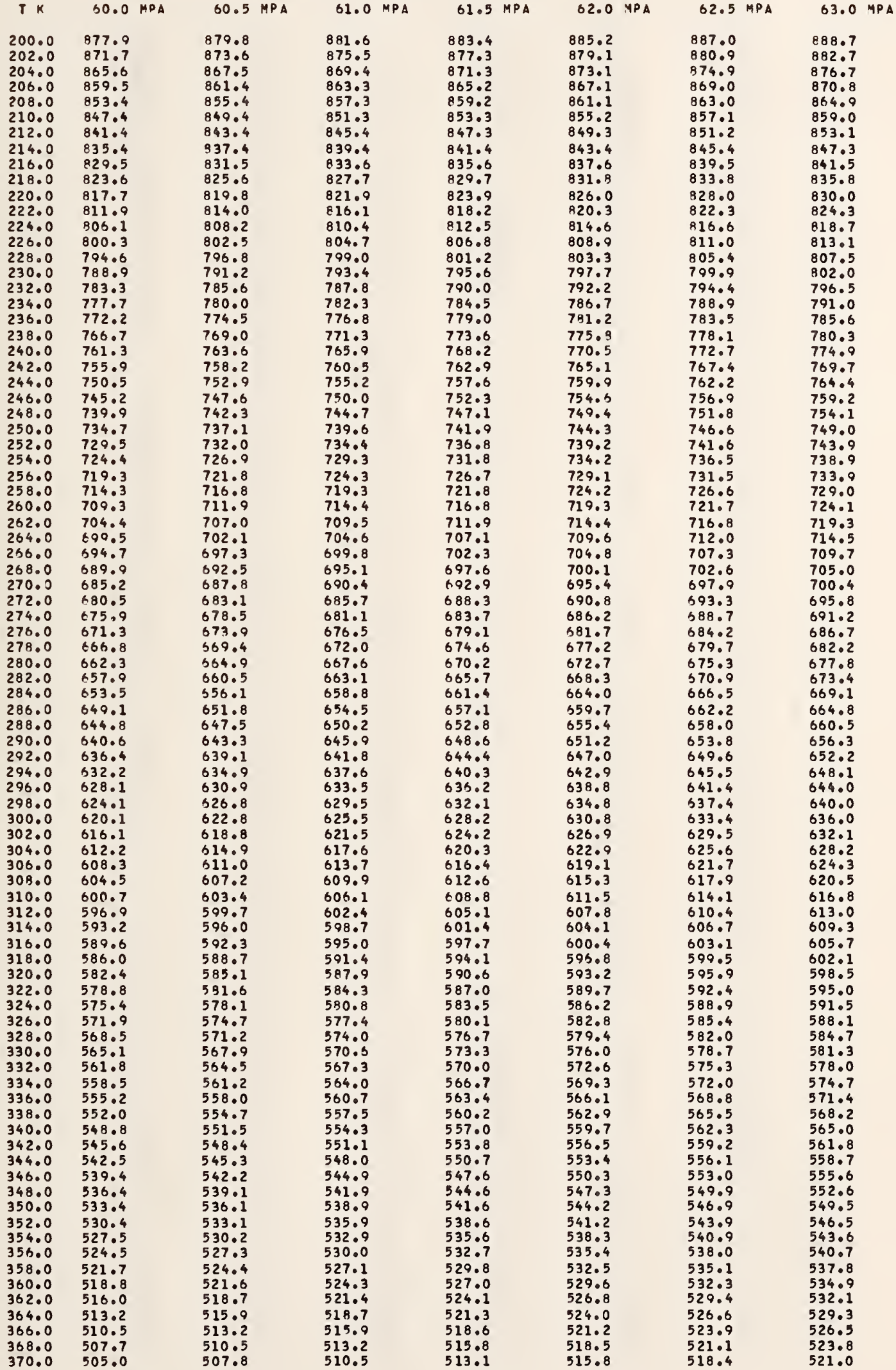


OXYGEN DENSITY (KG/M3).

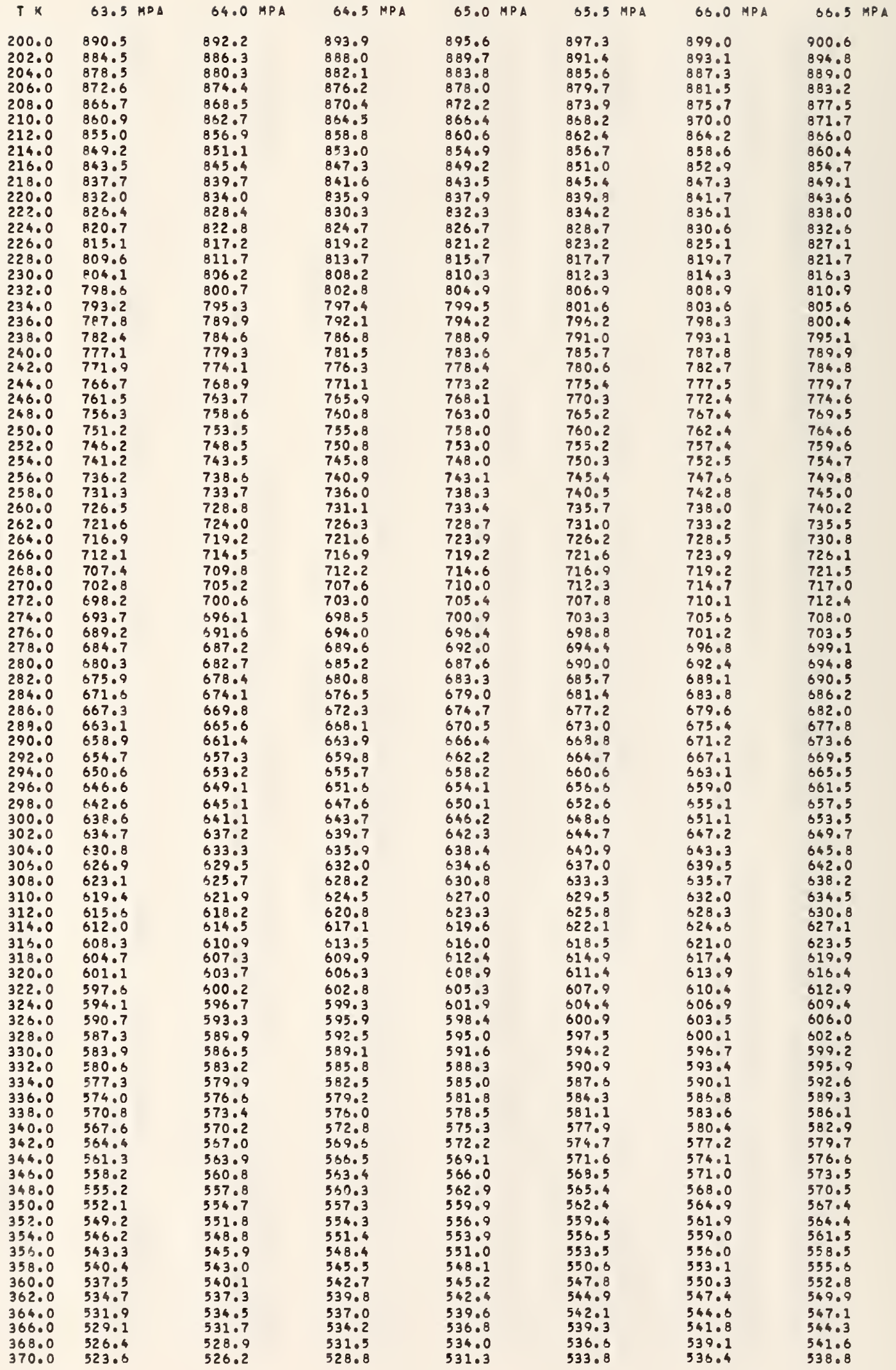


DXYGEN DENSITY (KG/MB).

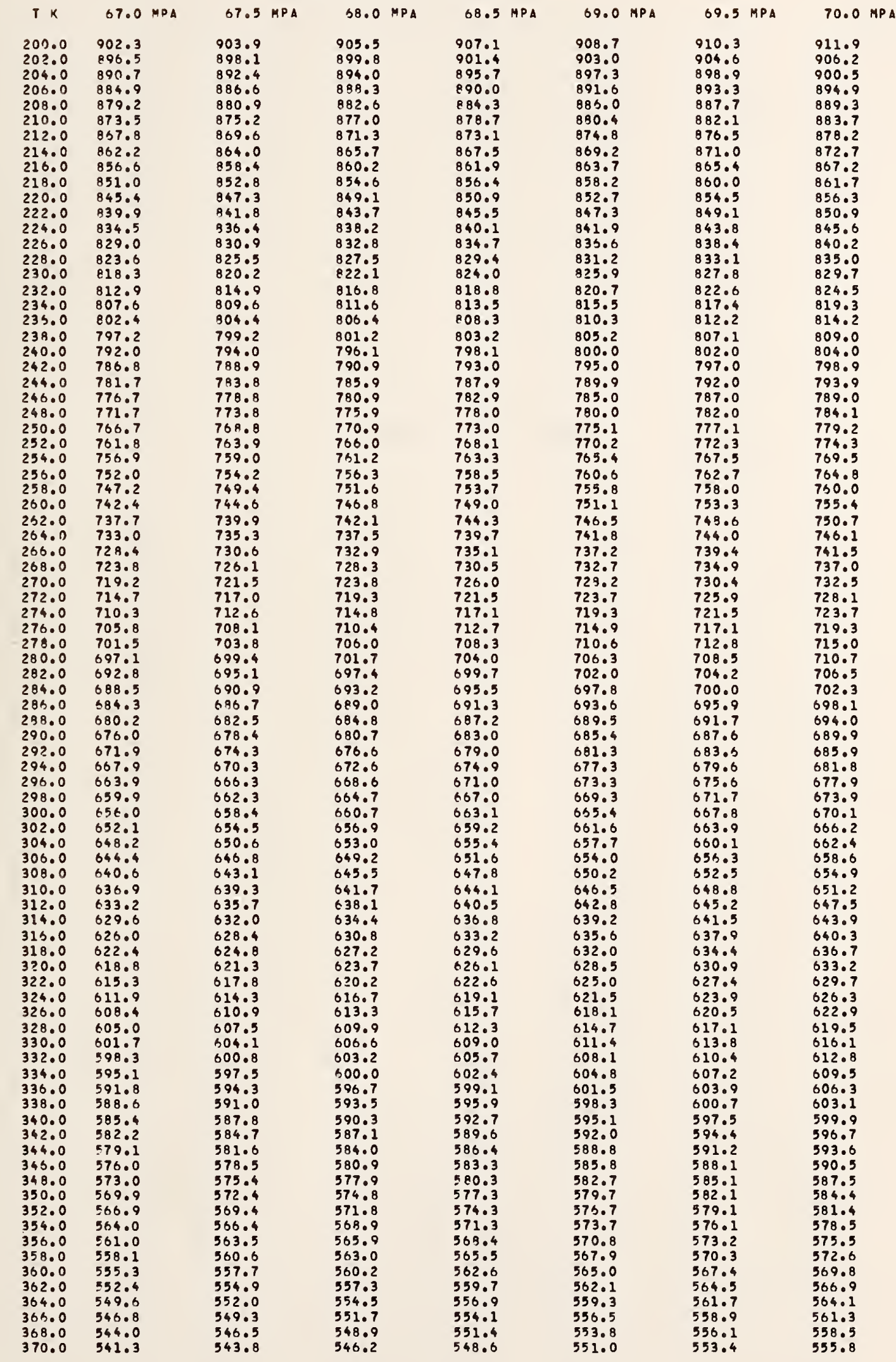




\section{2}

Argon Density $\left(\mathrm{kg} / \mathrm{m}^{3}\right)$ 
ARGON OENSITY (KG/MB).

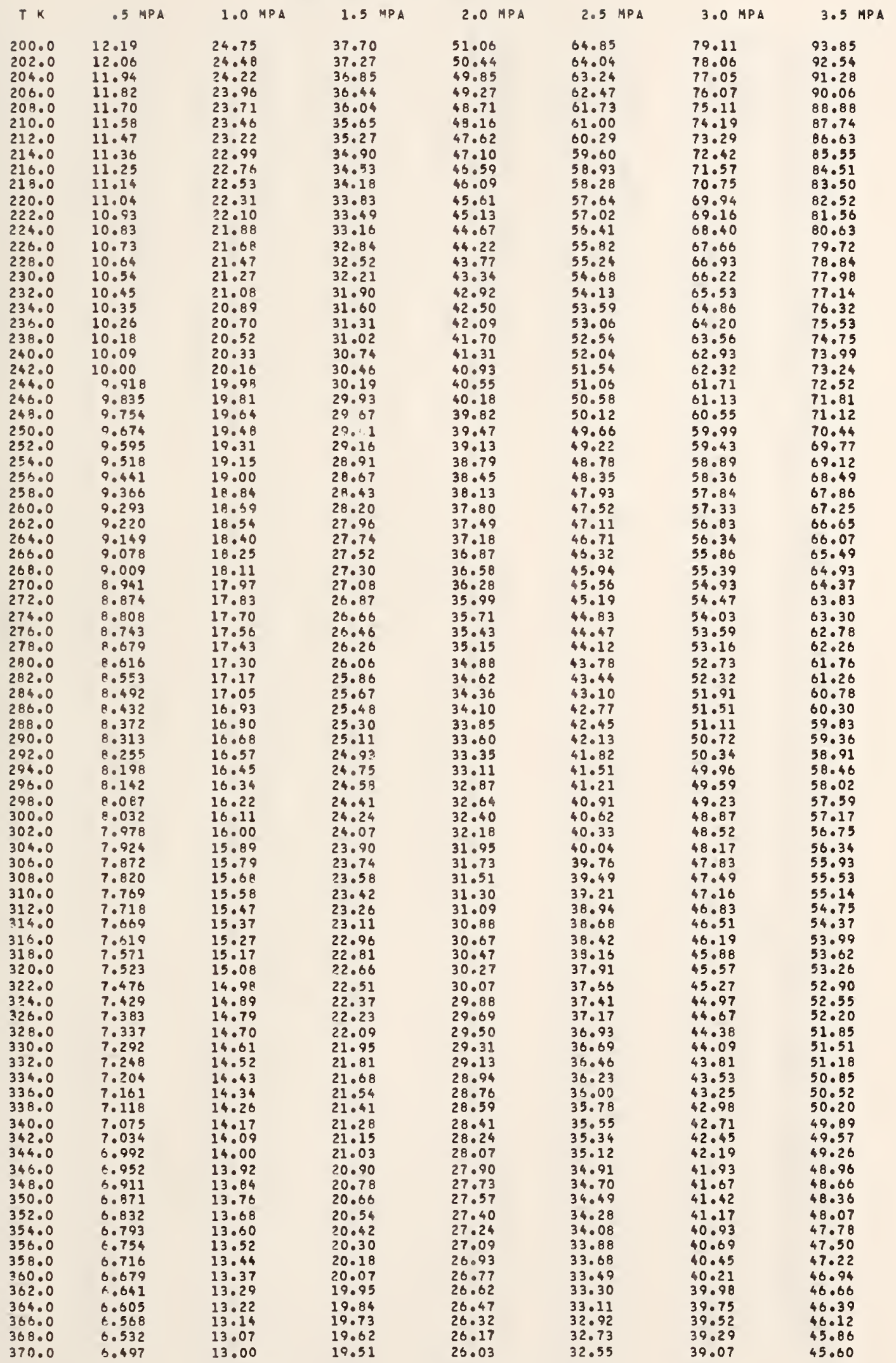


AQGON DENSITY (KG/M3).

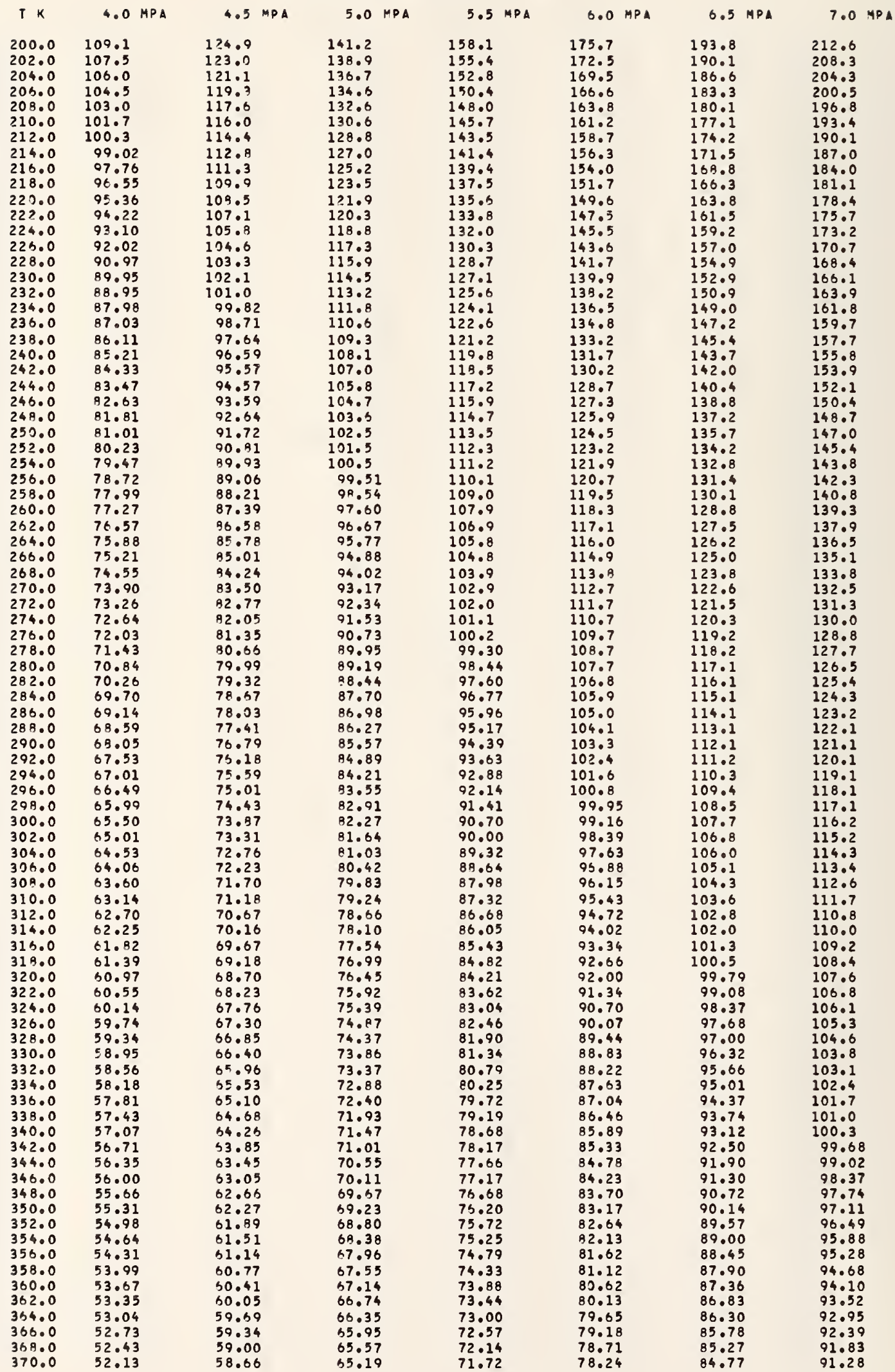


ARGON OENSITY (KG/MB).

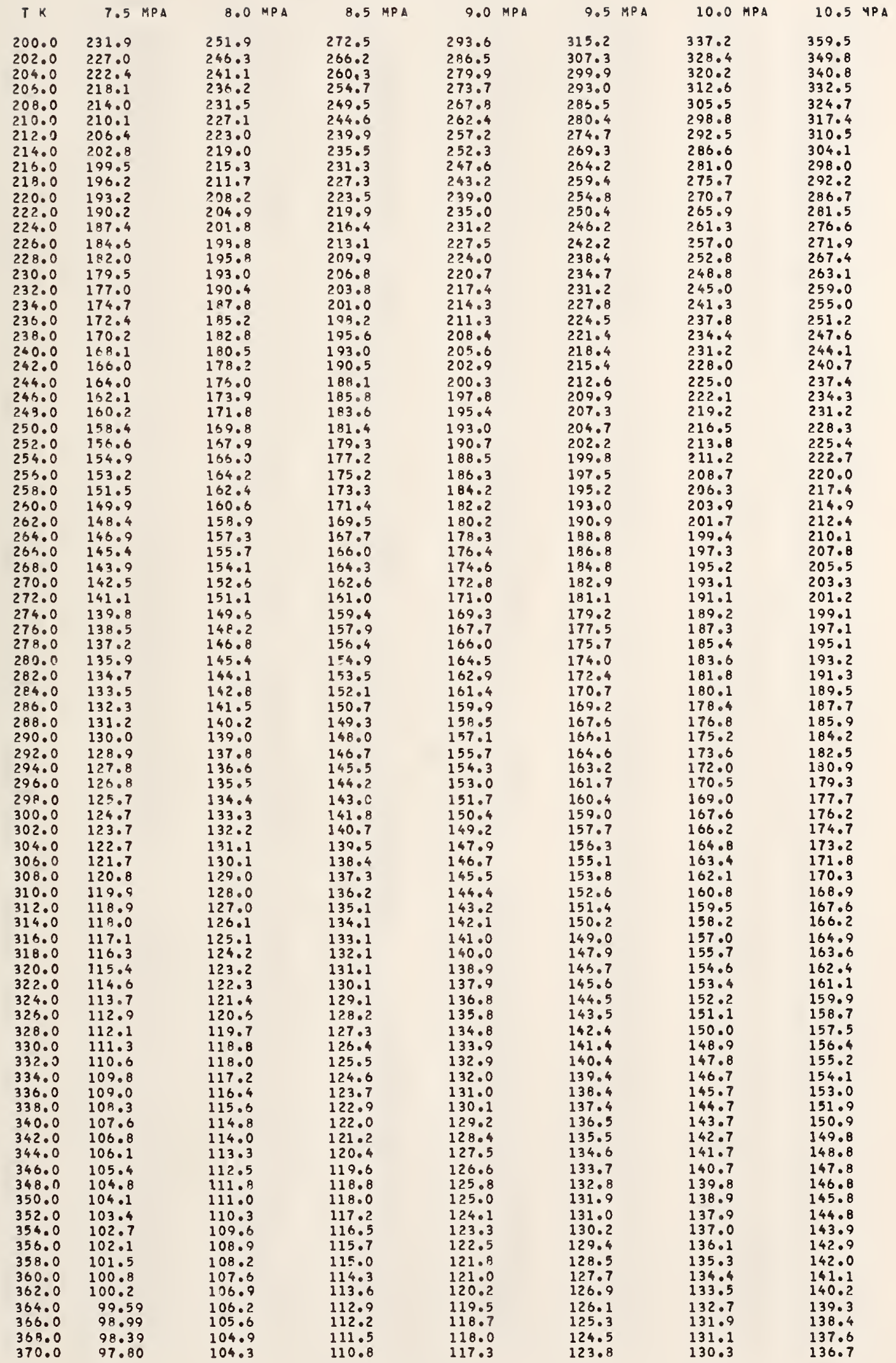


ARGIN OENSITY (KG/M3).

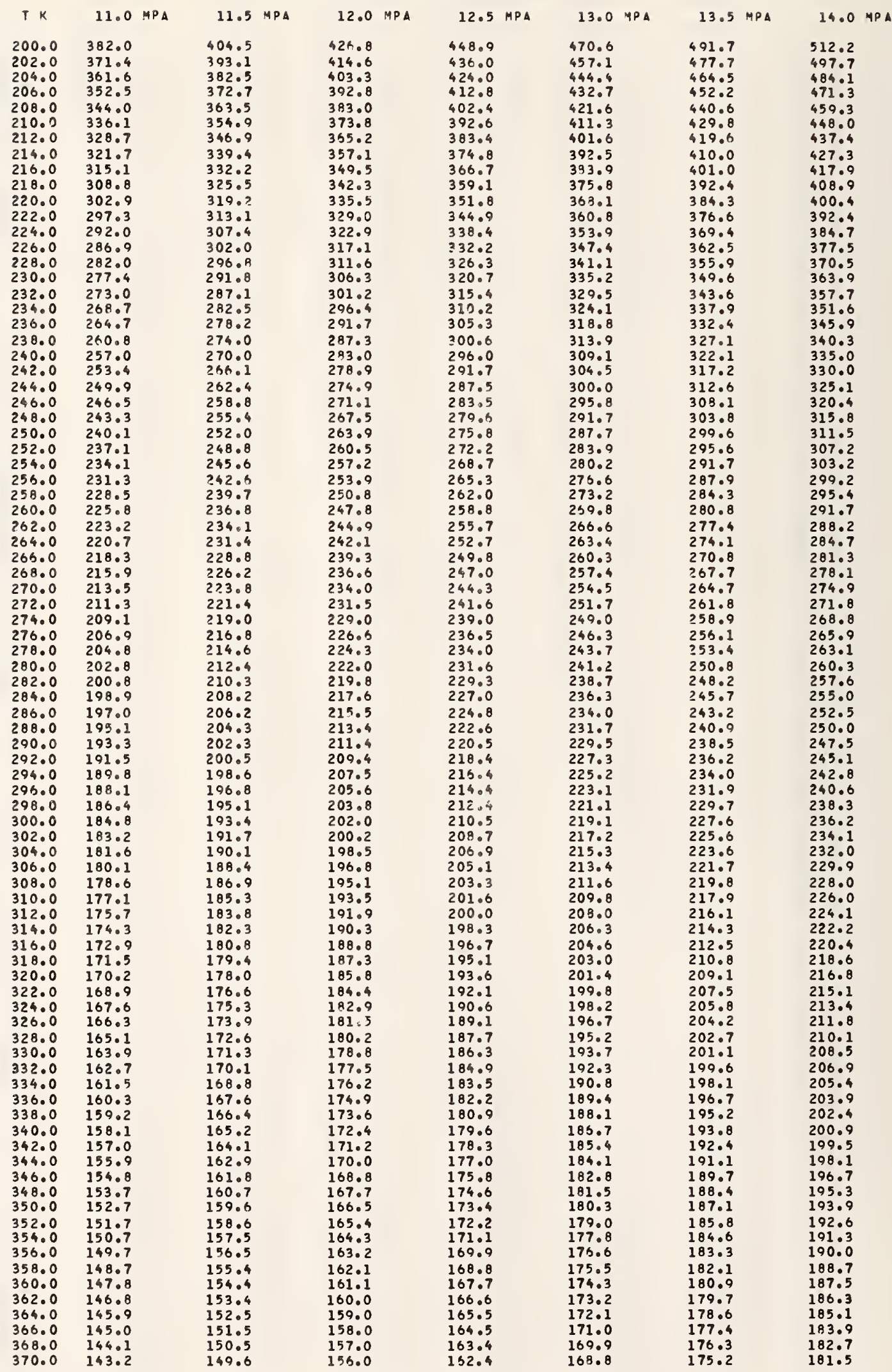


ARGON DENSITY (KG/M3).

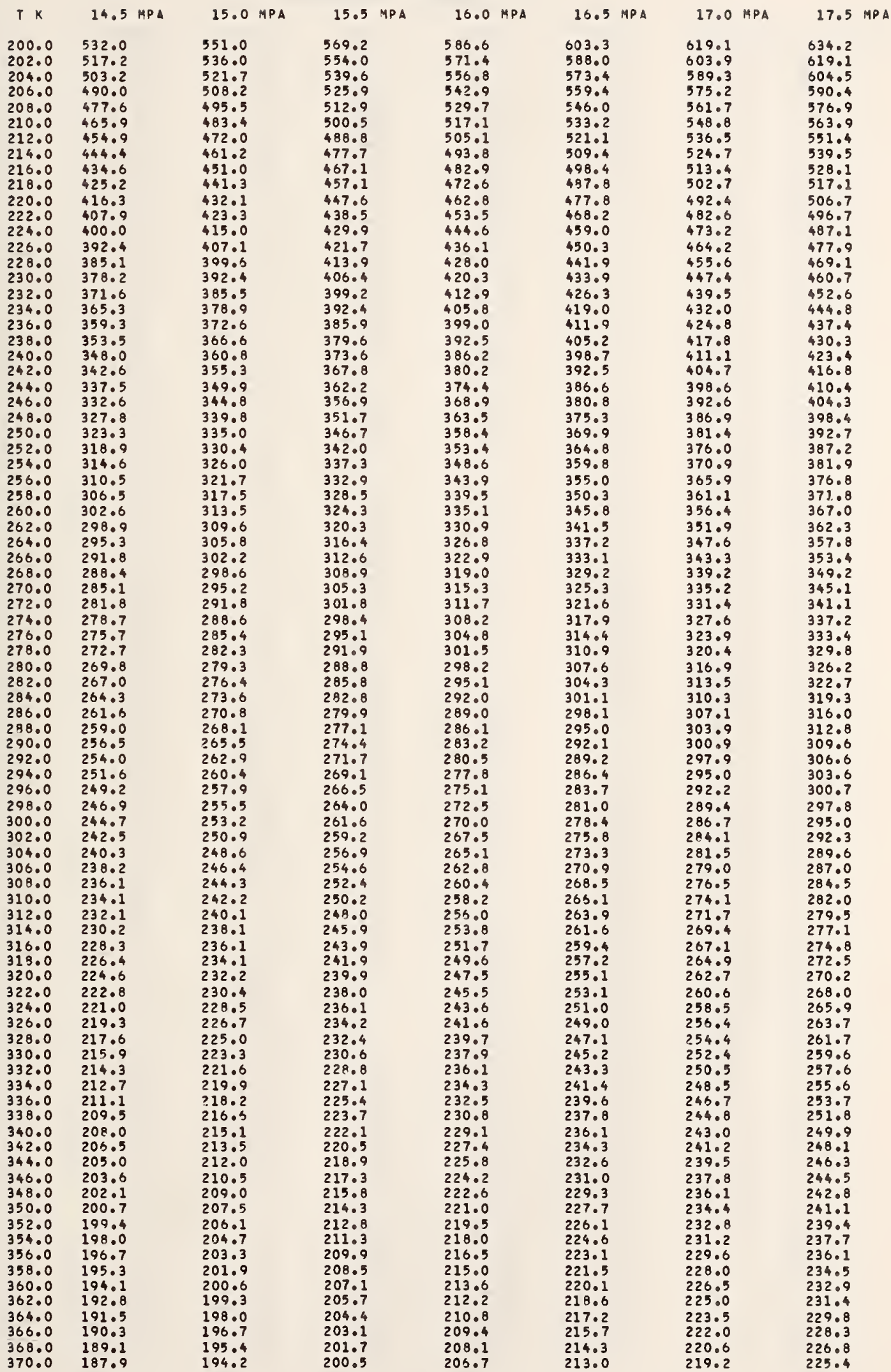


ARGON DENSITY (KG/H3).

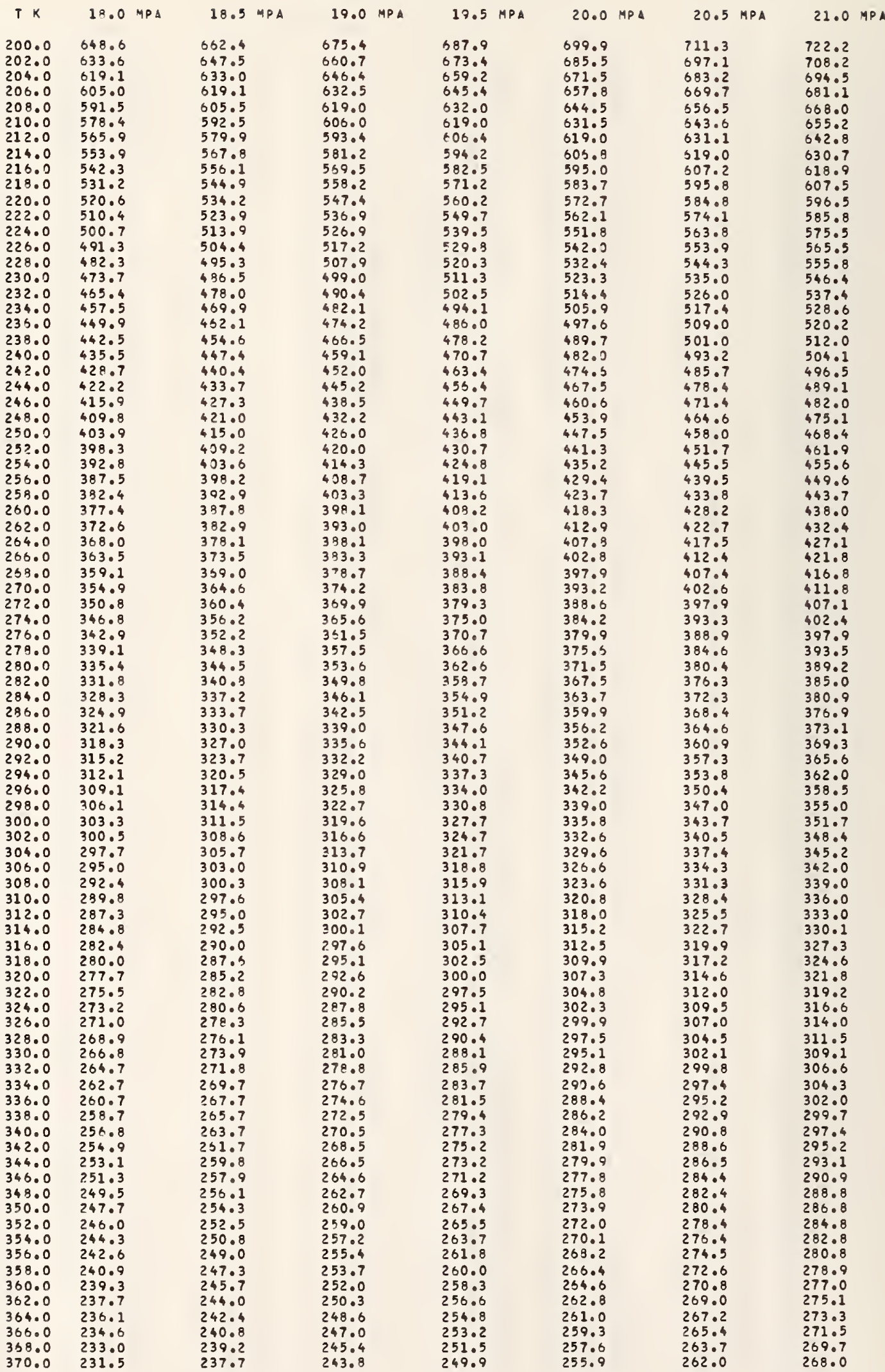


ARGON OENSITY (KG/M3).

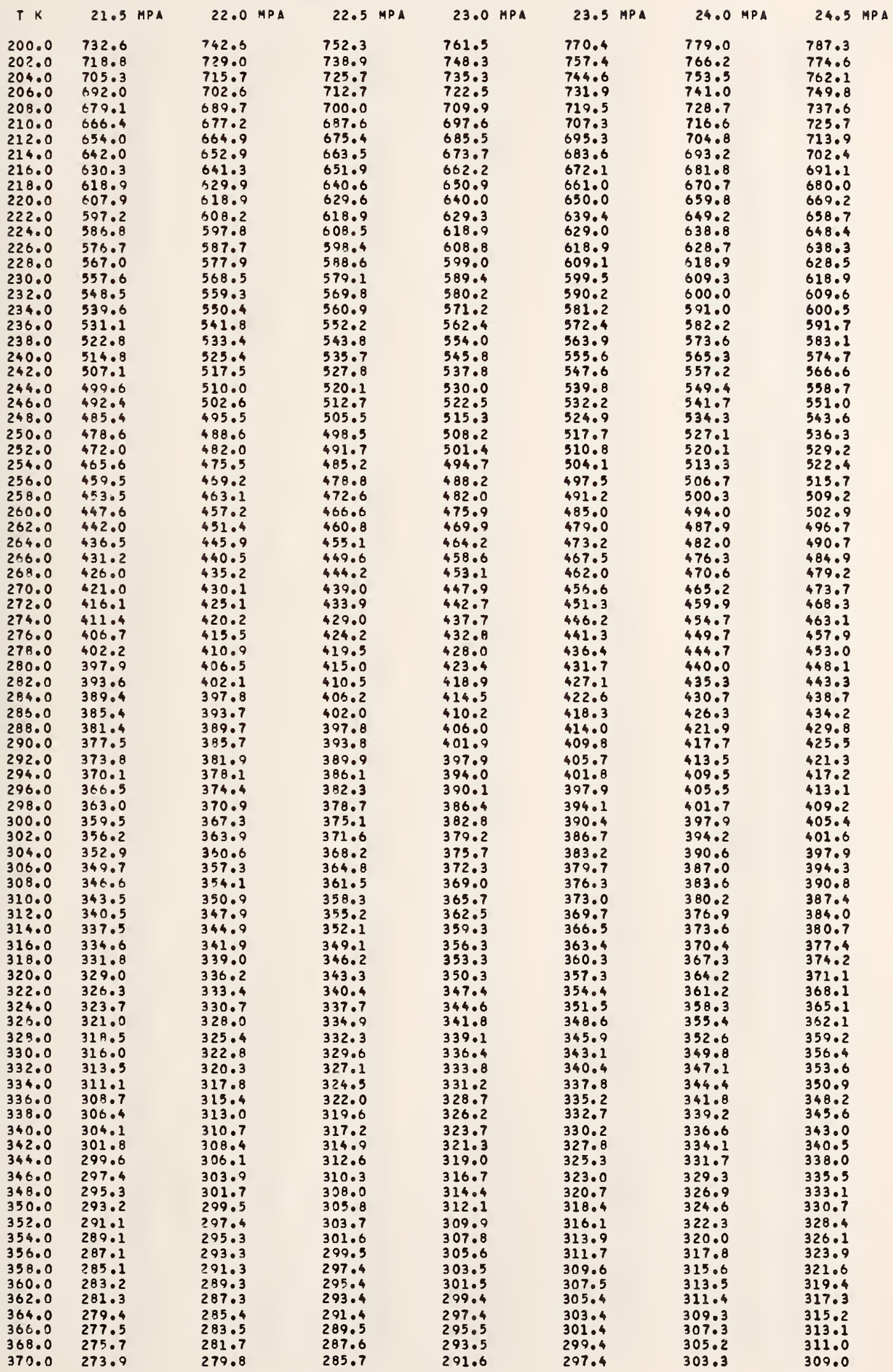


ARGON OENSITY (KG/M3).

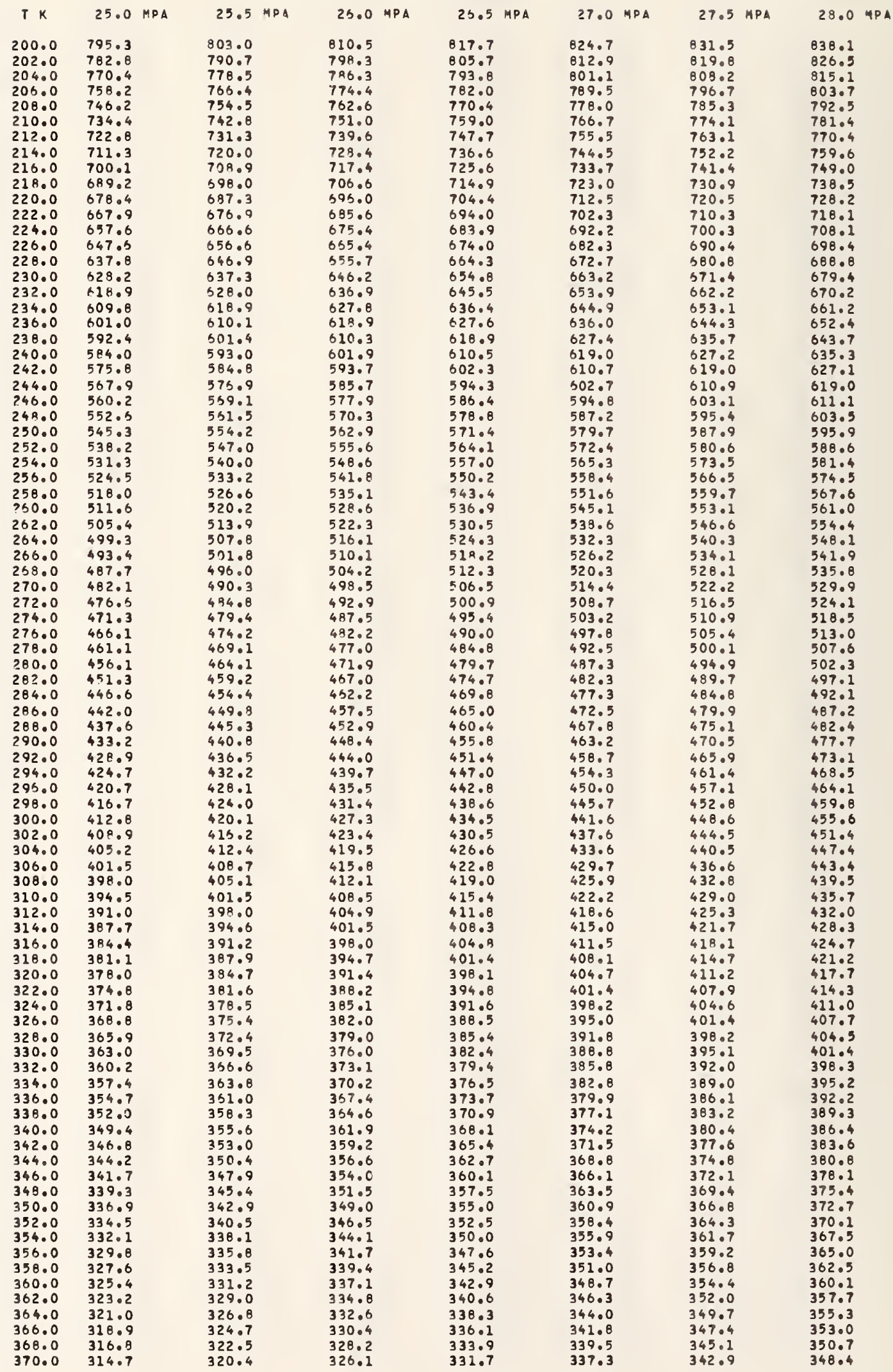


ARGON DENSITY (KG/MB).

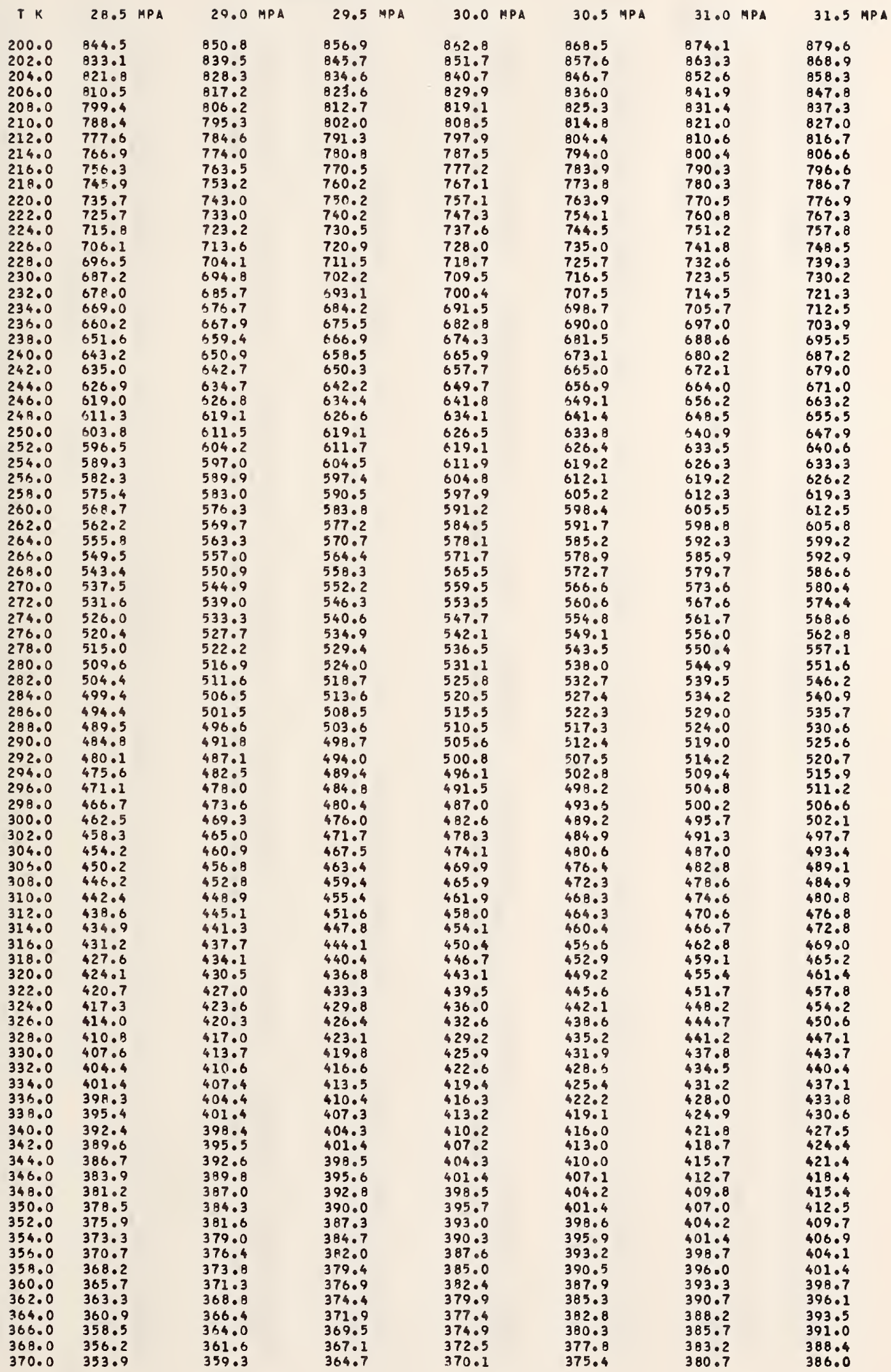


ARGCN OENSITY (KG/MB).

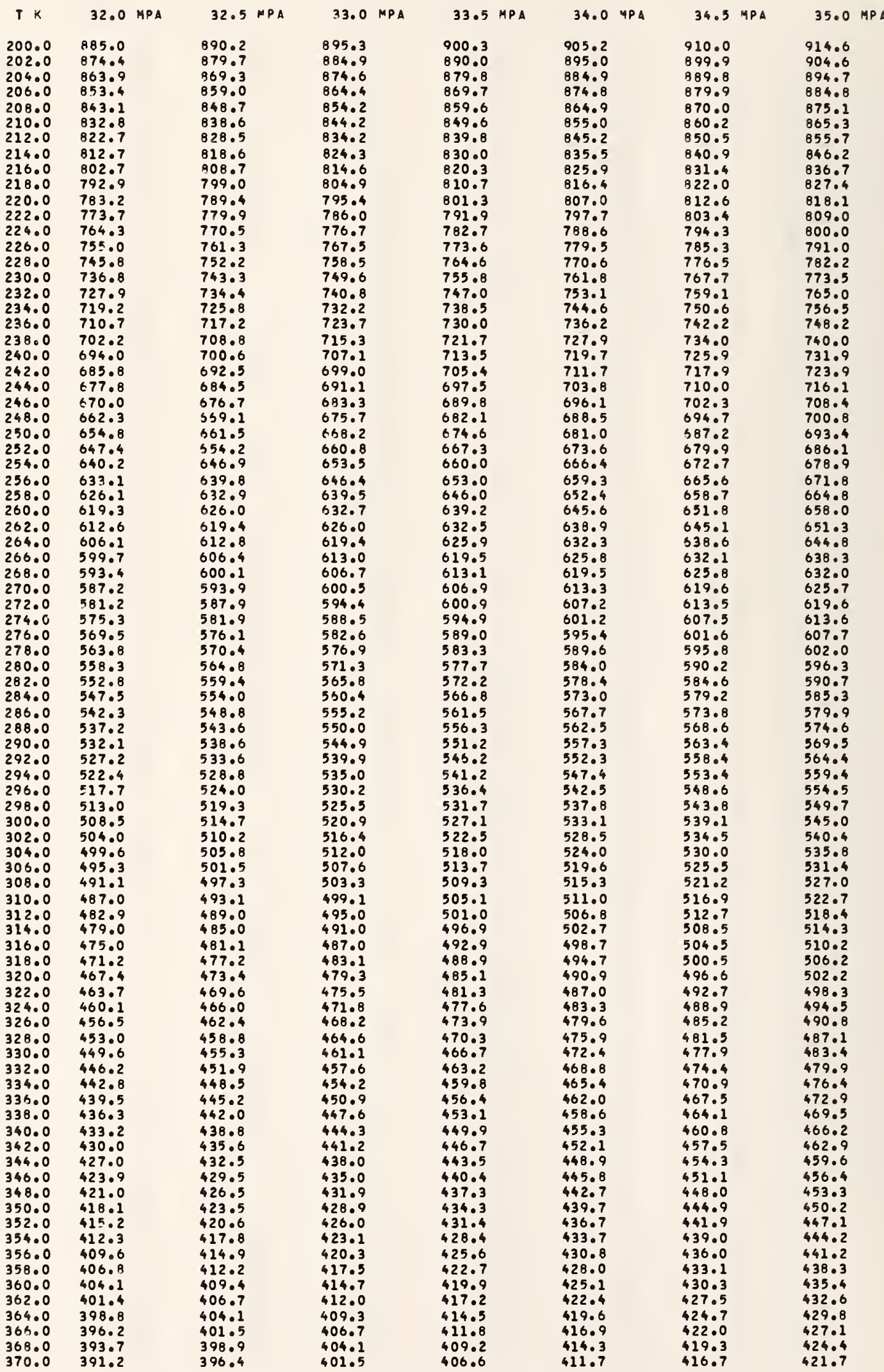


ARGON OENSITY (KG/M3).

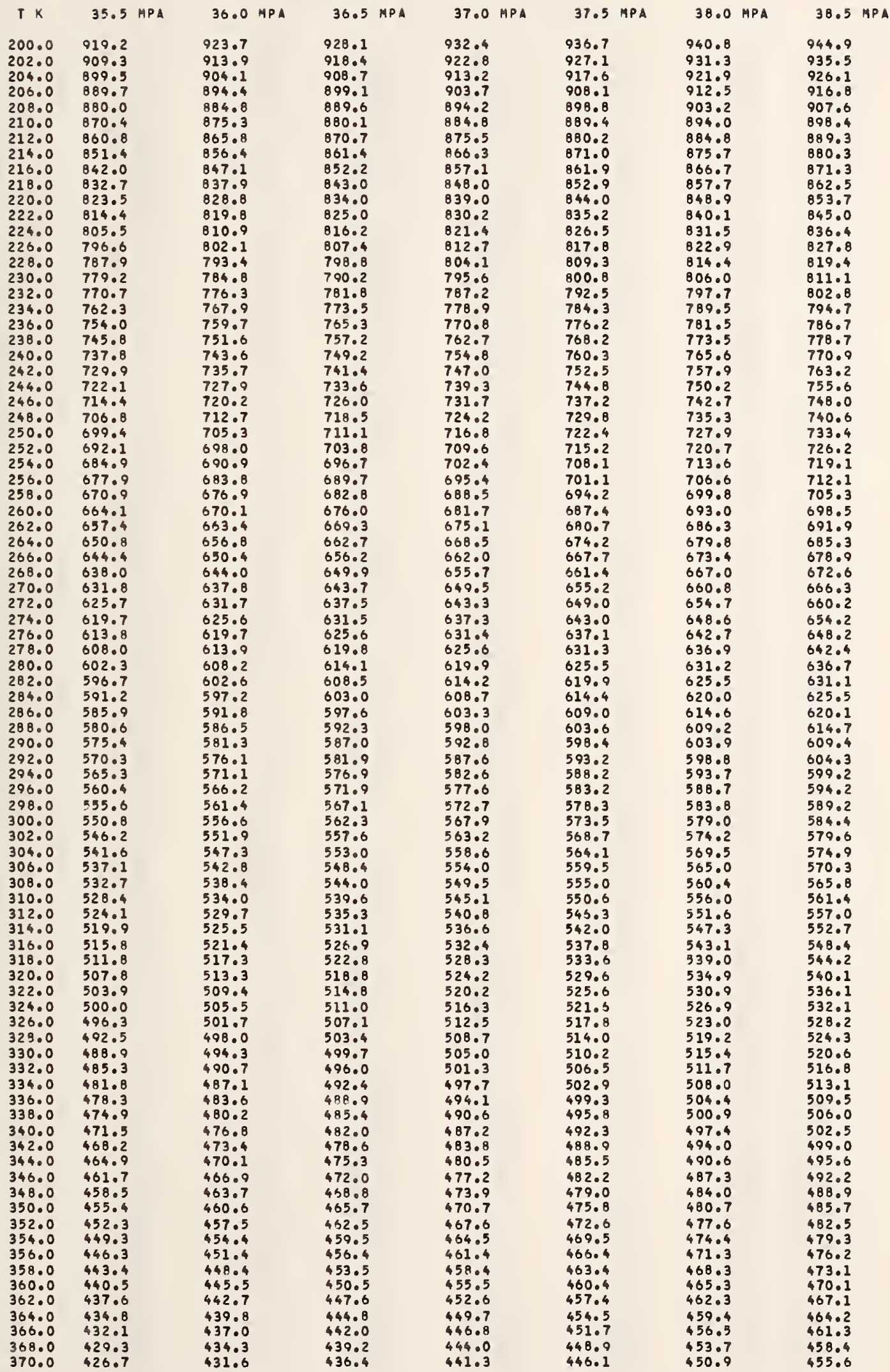


$\triangle R G C N$ DENSITY (KG/M3).

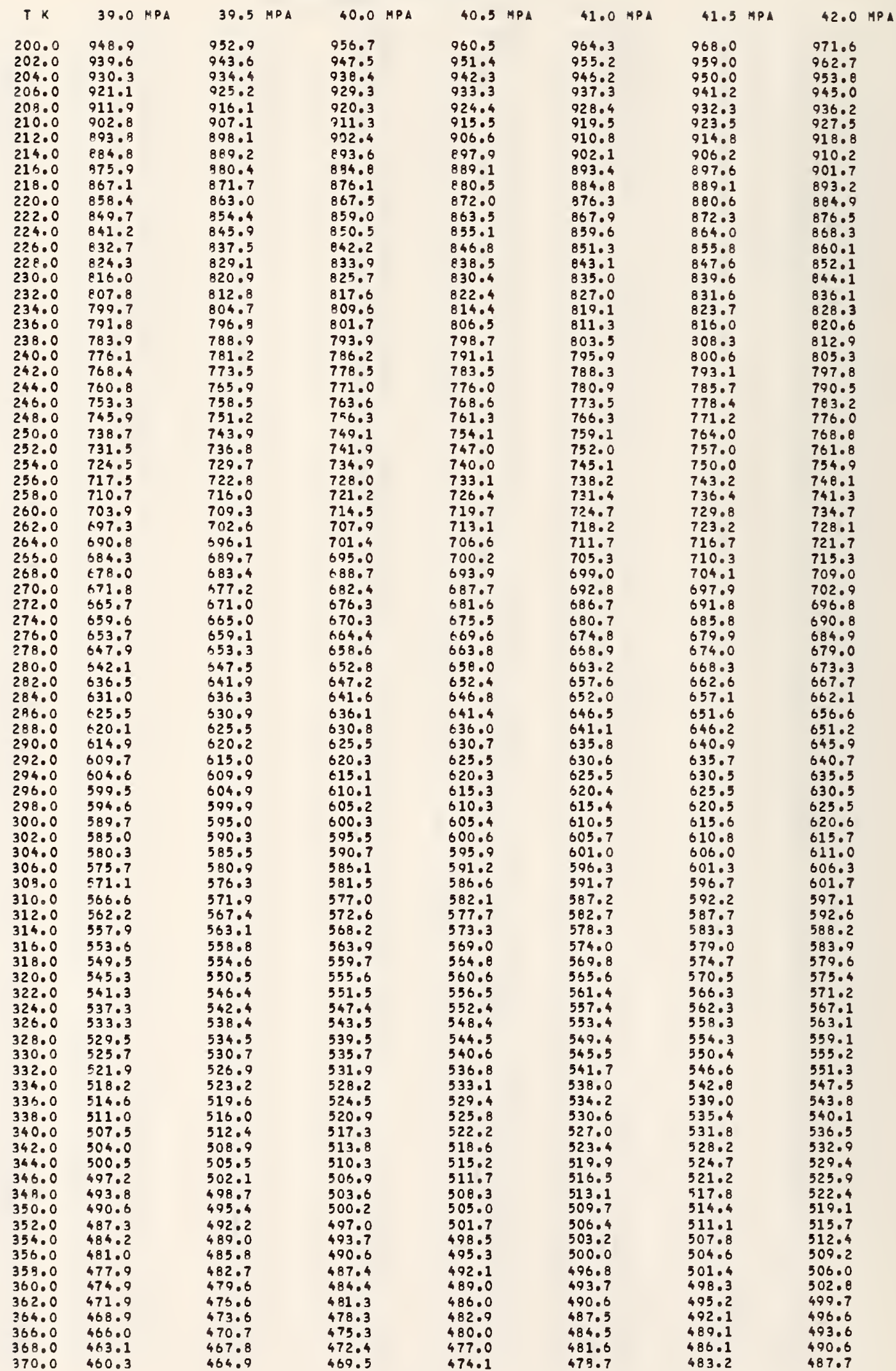


ARGON DENSITY (KG/H3).

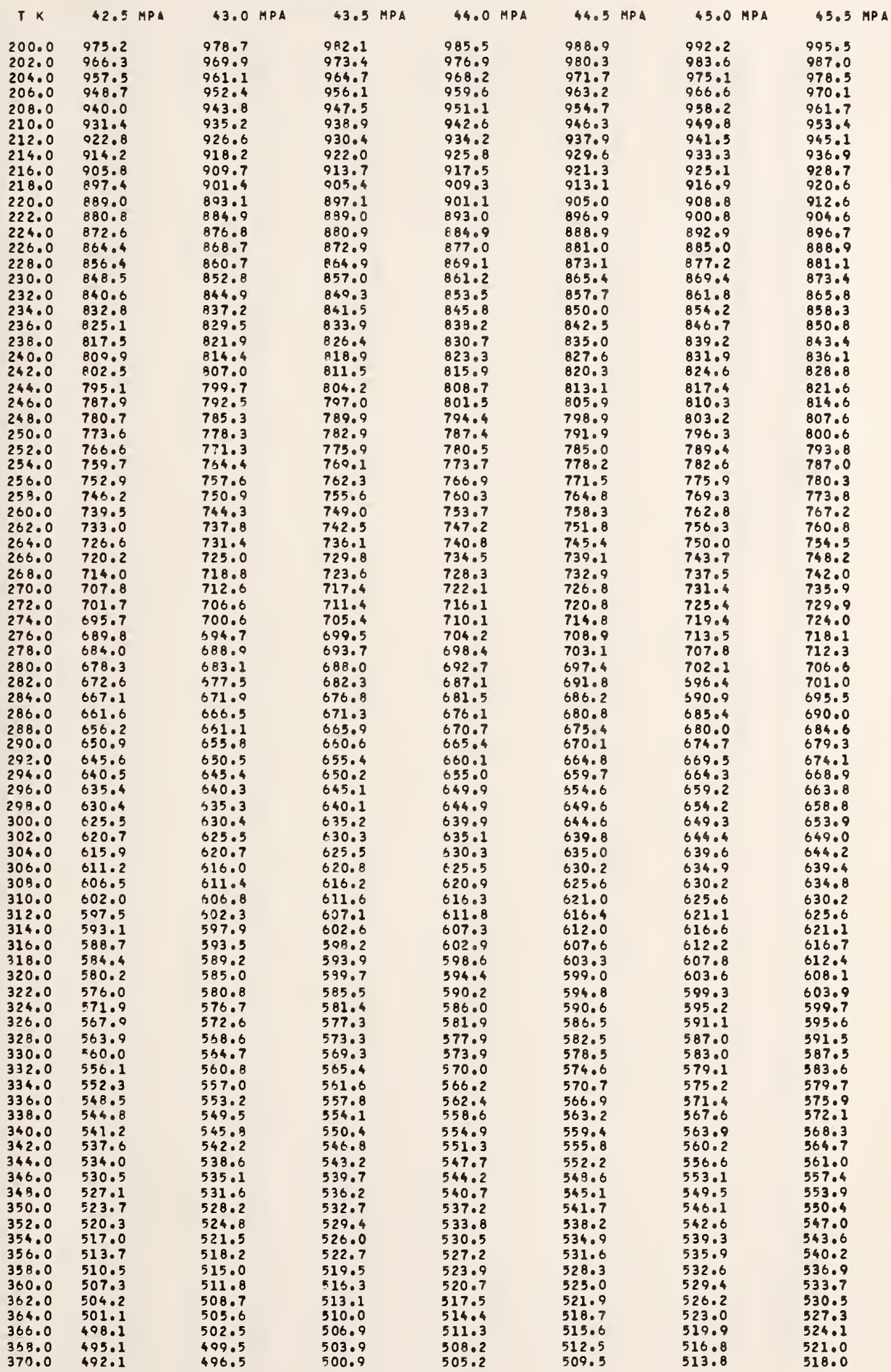


ARGON DENSITY (KG/M3).

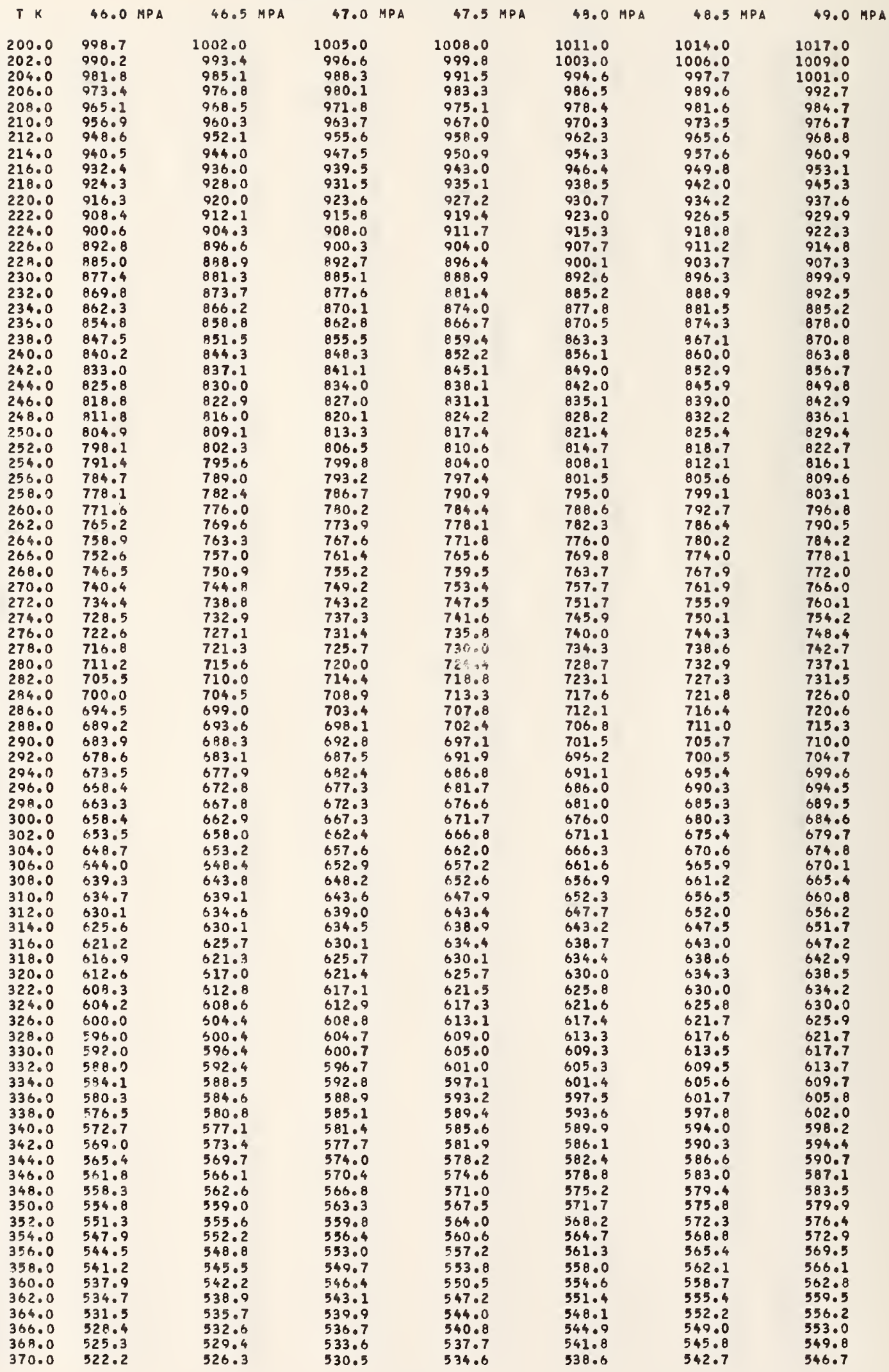


ARGON DENSITY (KGIMB).

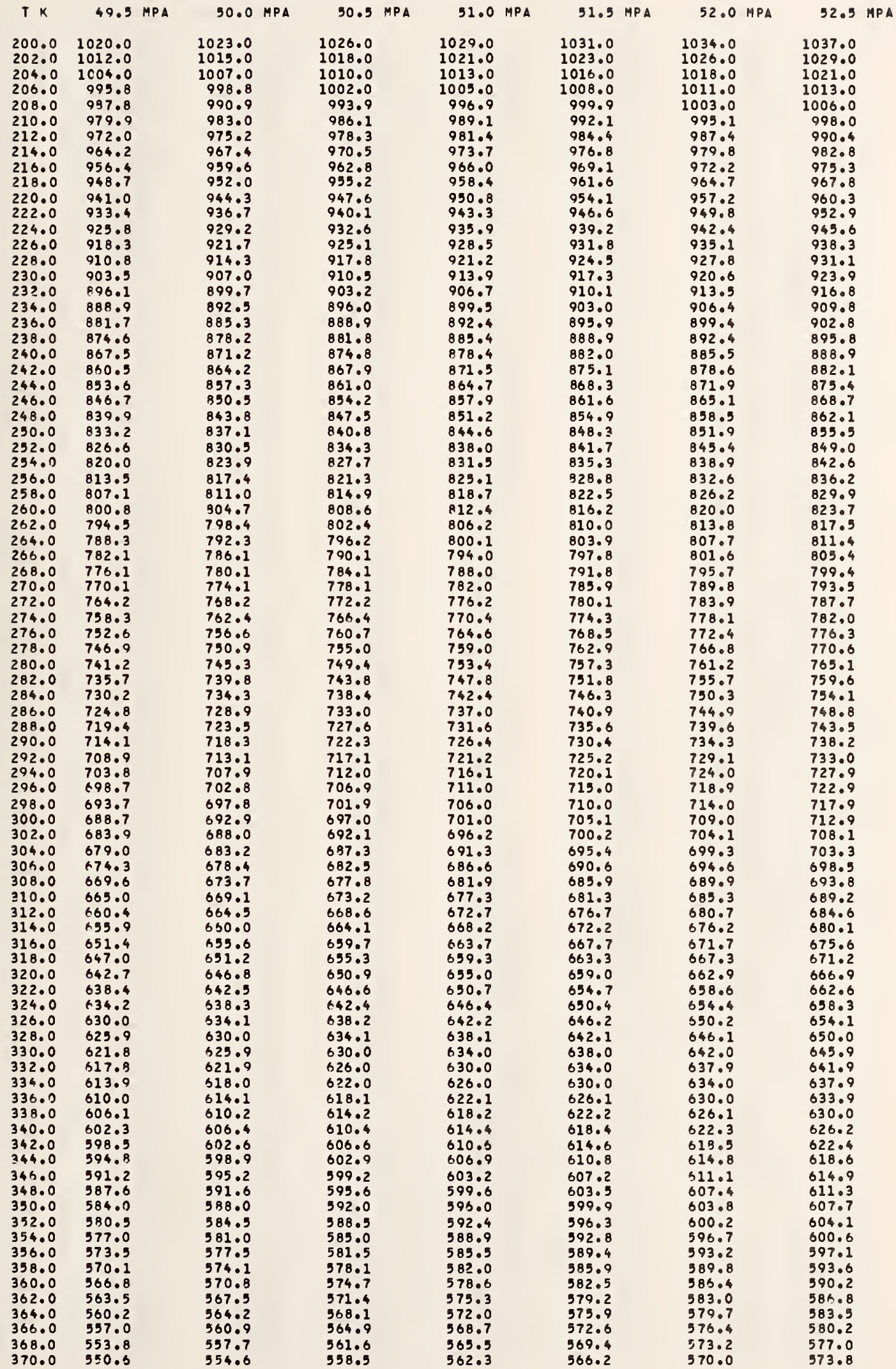


ARGON OENSITY (KGIMG).

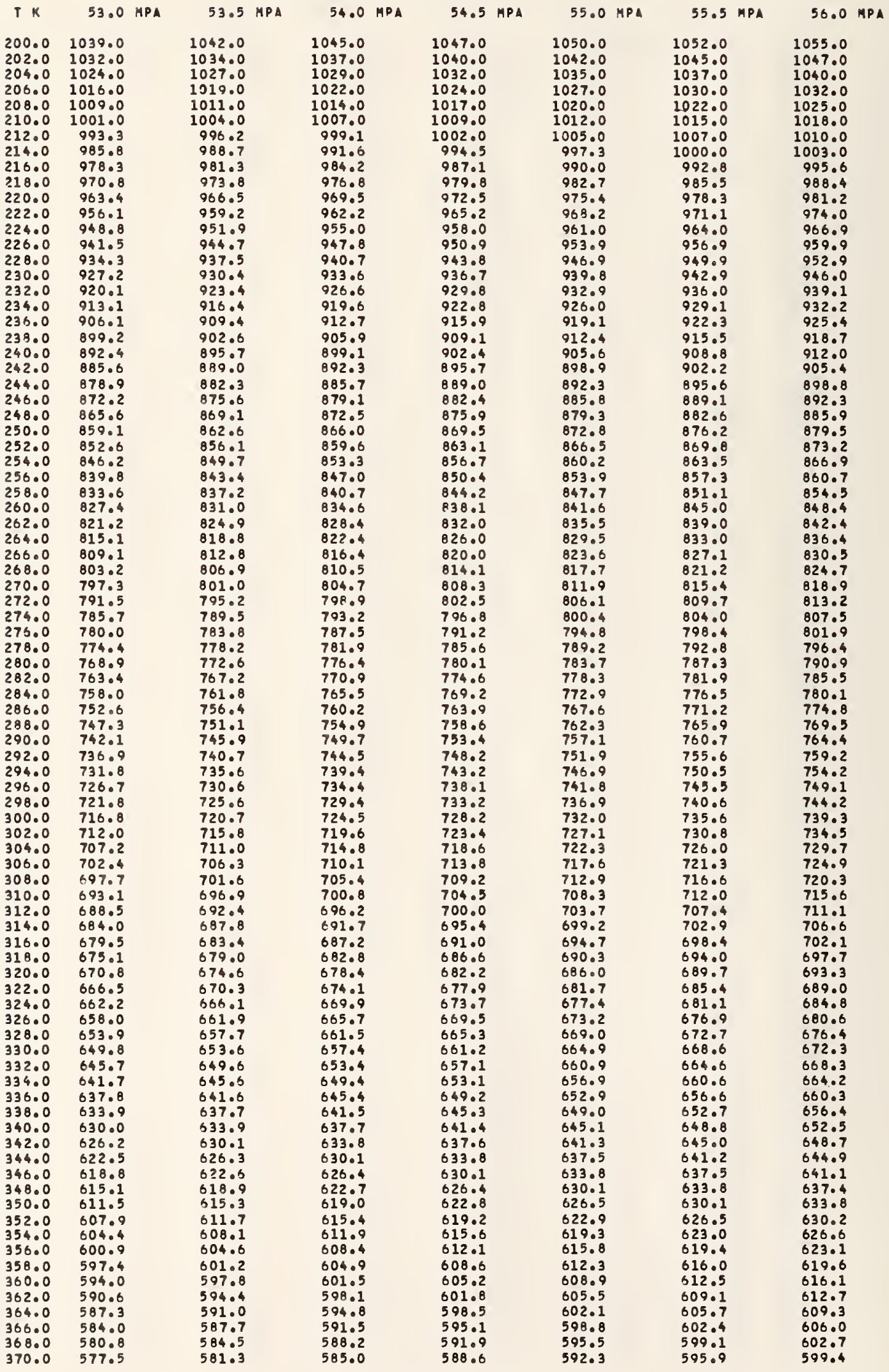


ARGON DENSIYY (KG/M3).

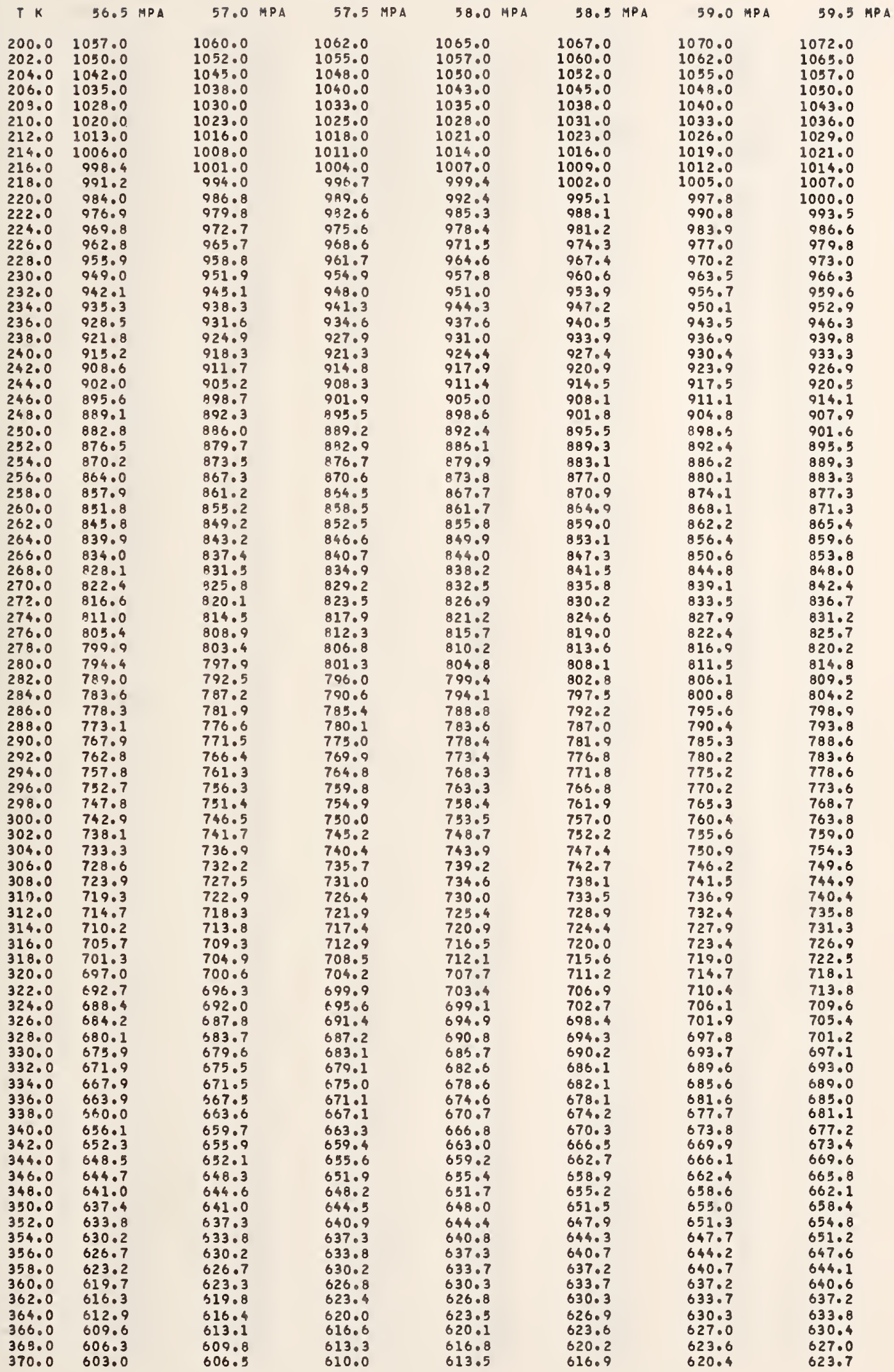


ARGCN DENSITY (KG/M3).

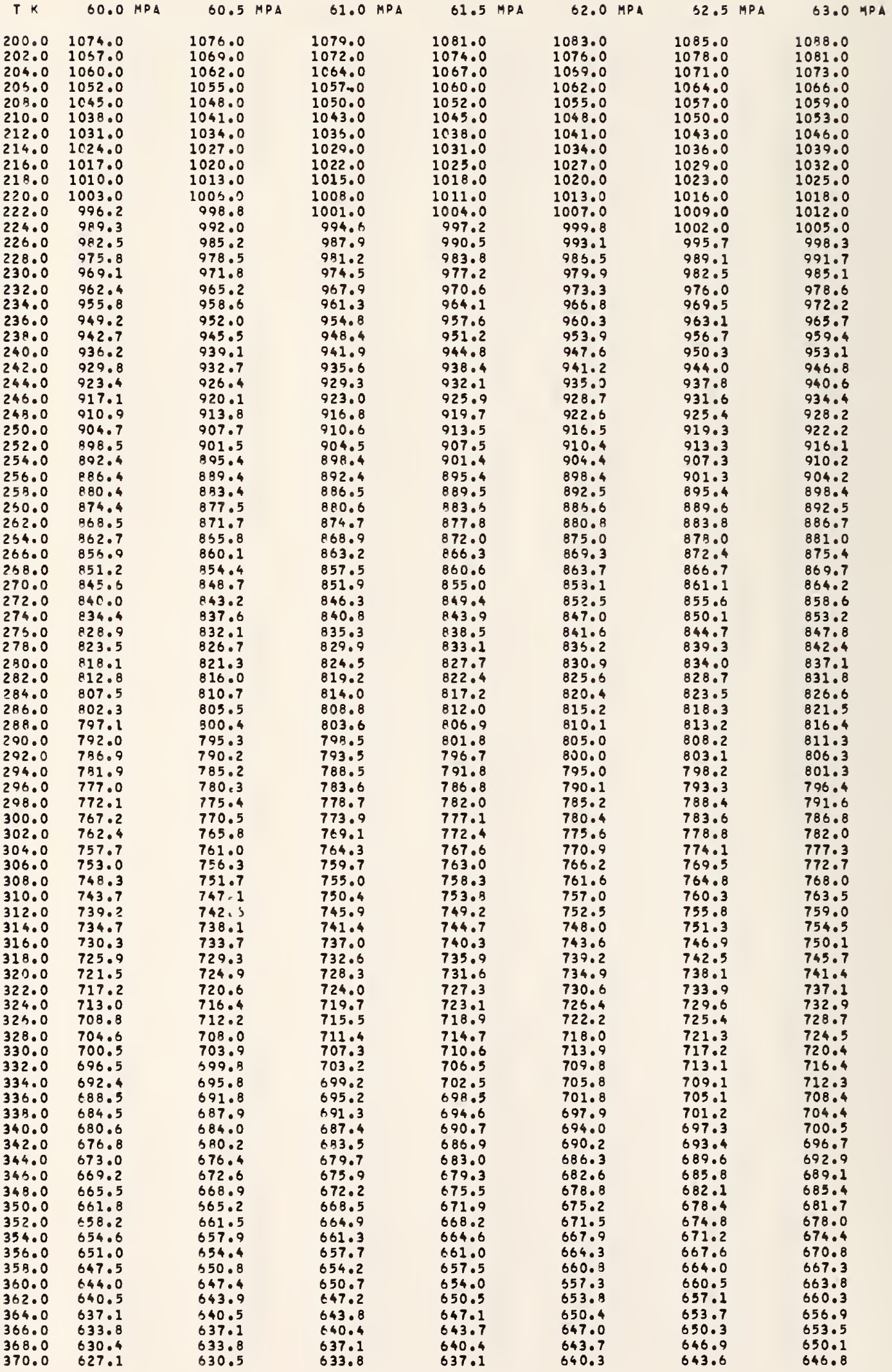


ARGON OENSITY (KG/M3).

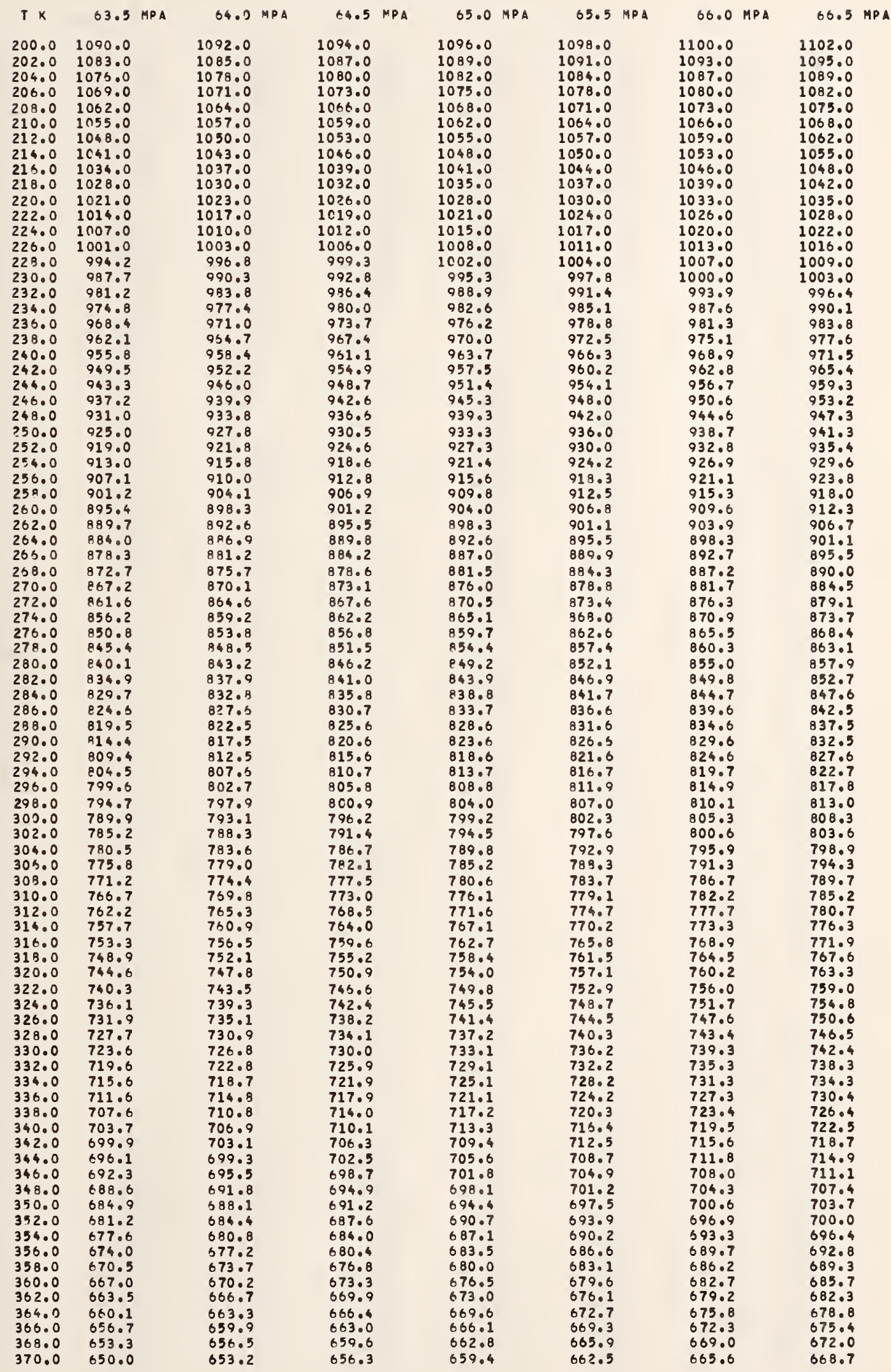


ARGOM OEHSITY (KG/HZ).

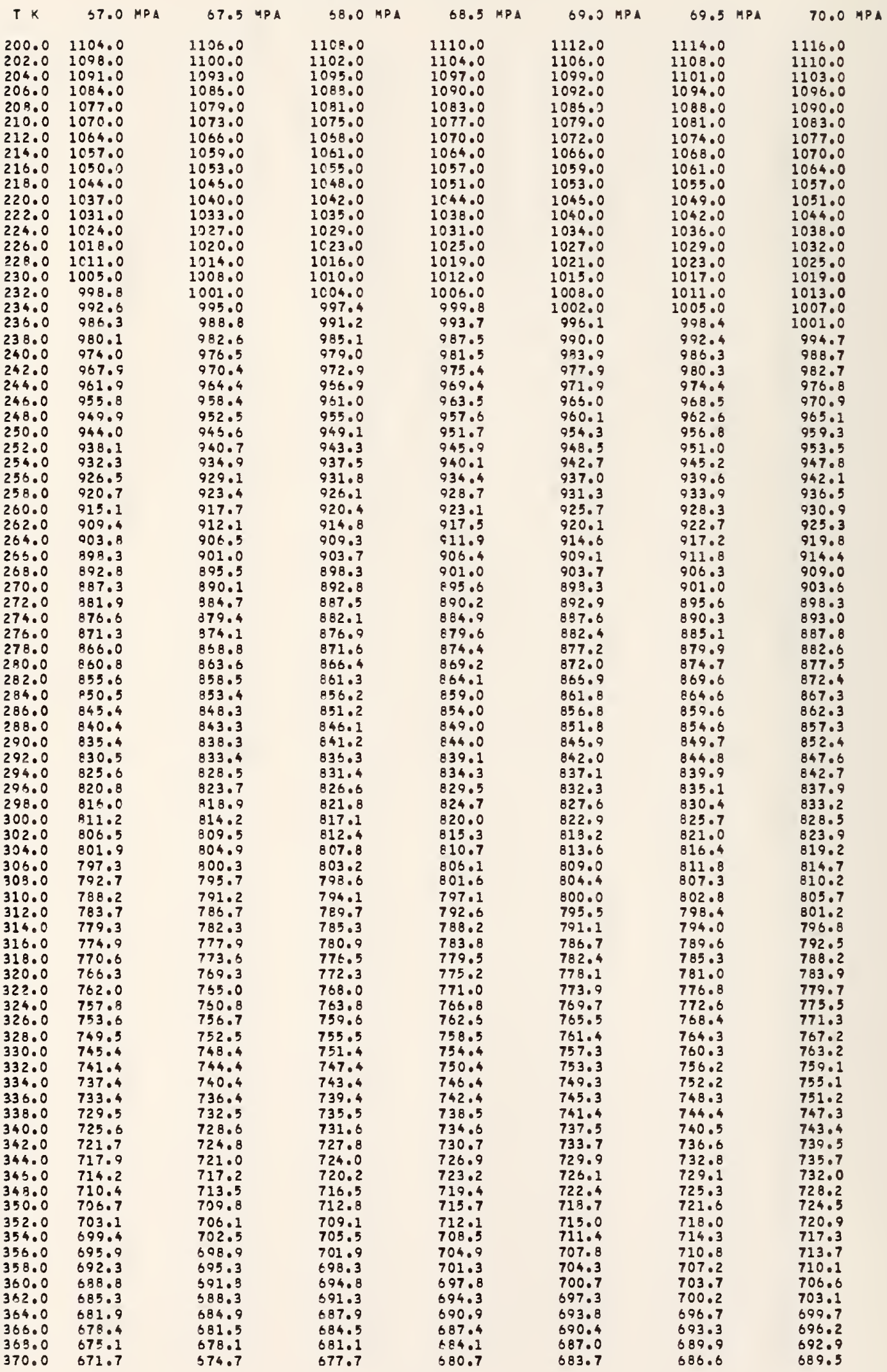


9.3

Nitrogen Density $\left(\mathrm{kg} / \mathrm{m}^{3}\right)$ 
NITROGEN DENSITY (KG/M3).

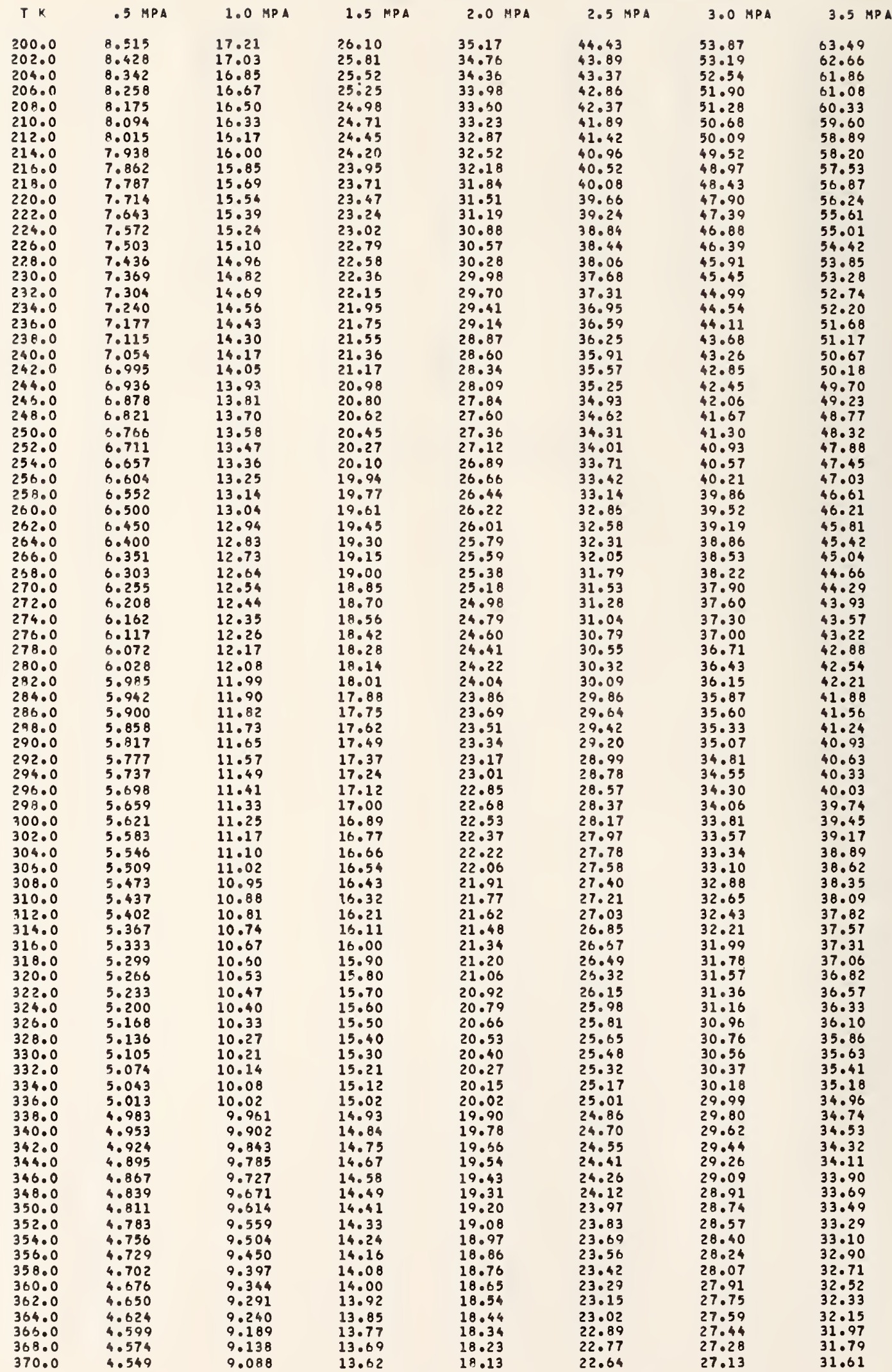


NITROGEN DENSITY (KG/M3).

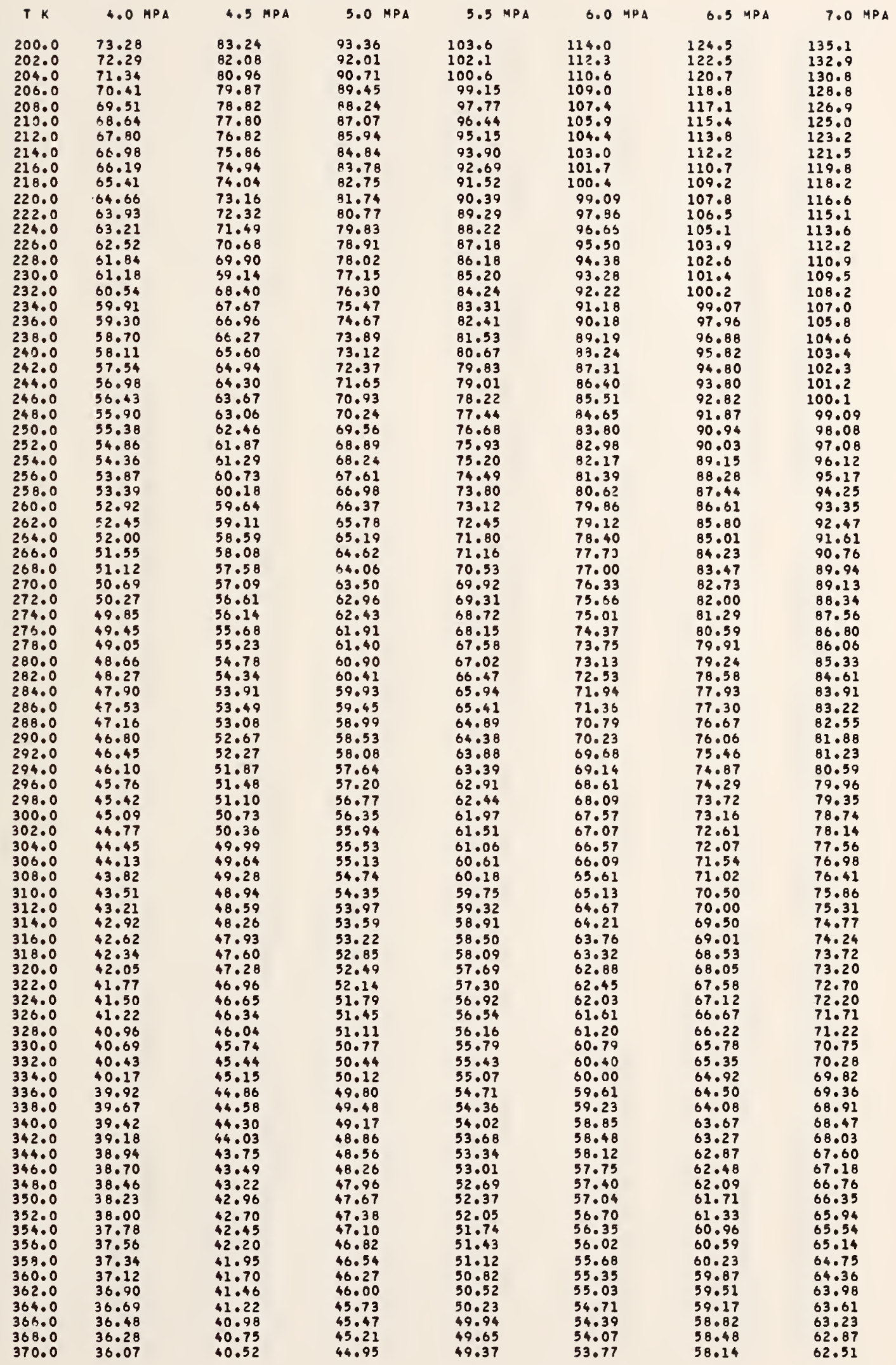


NITROGEN OENSITY (KG/M3).

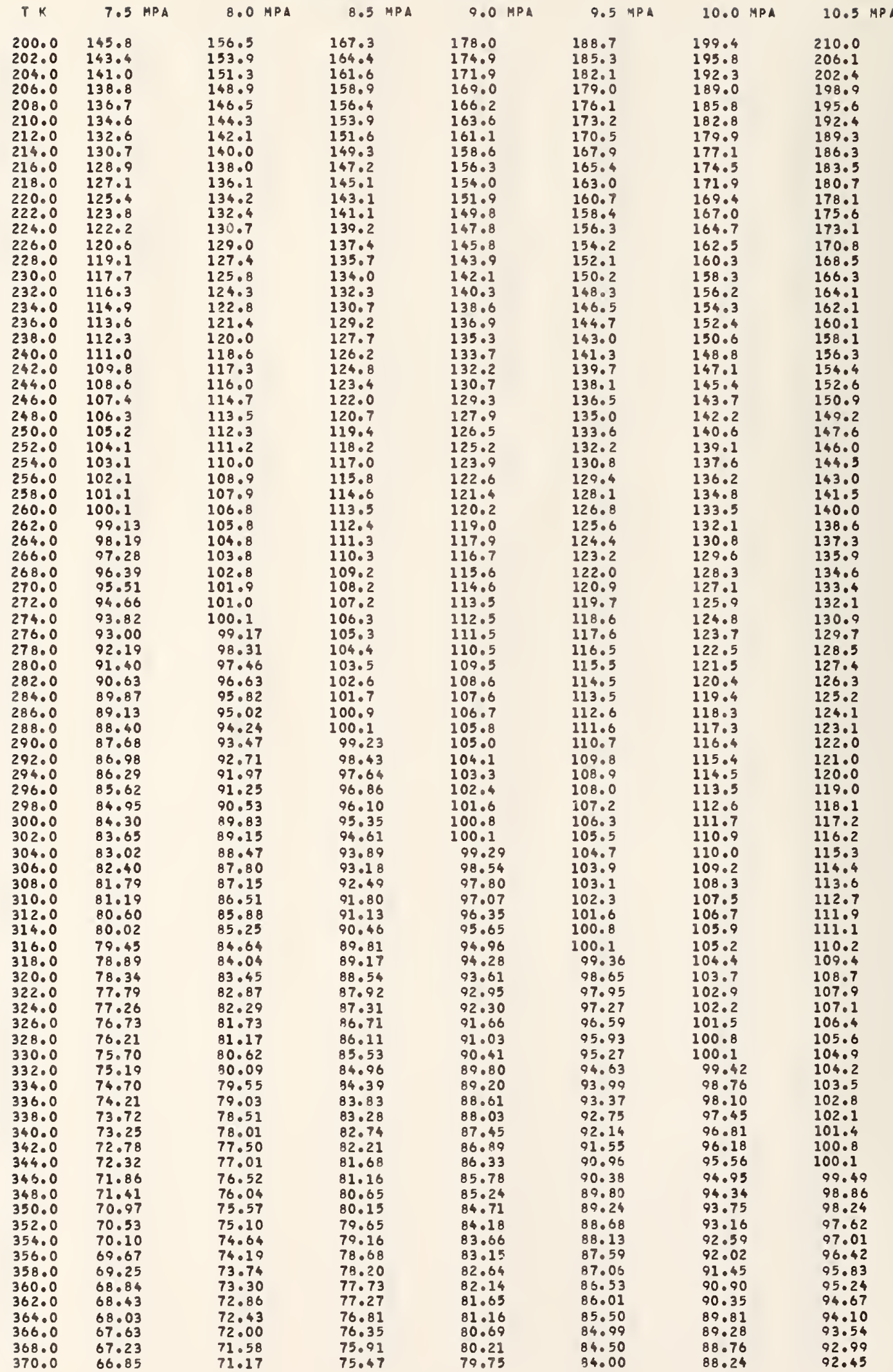


NITROGEN DENSITY (KG/M3).

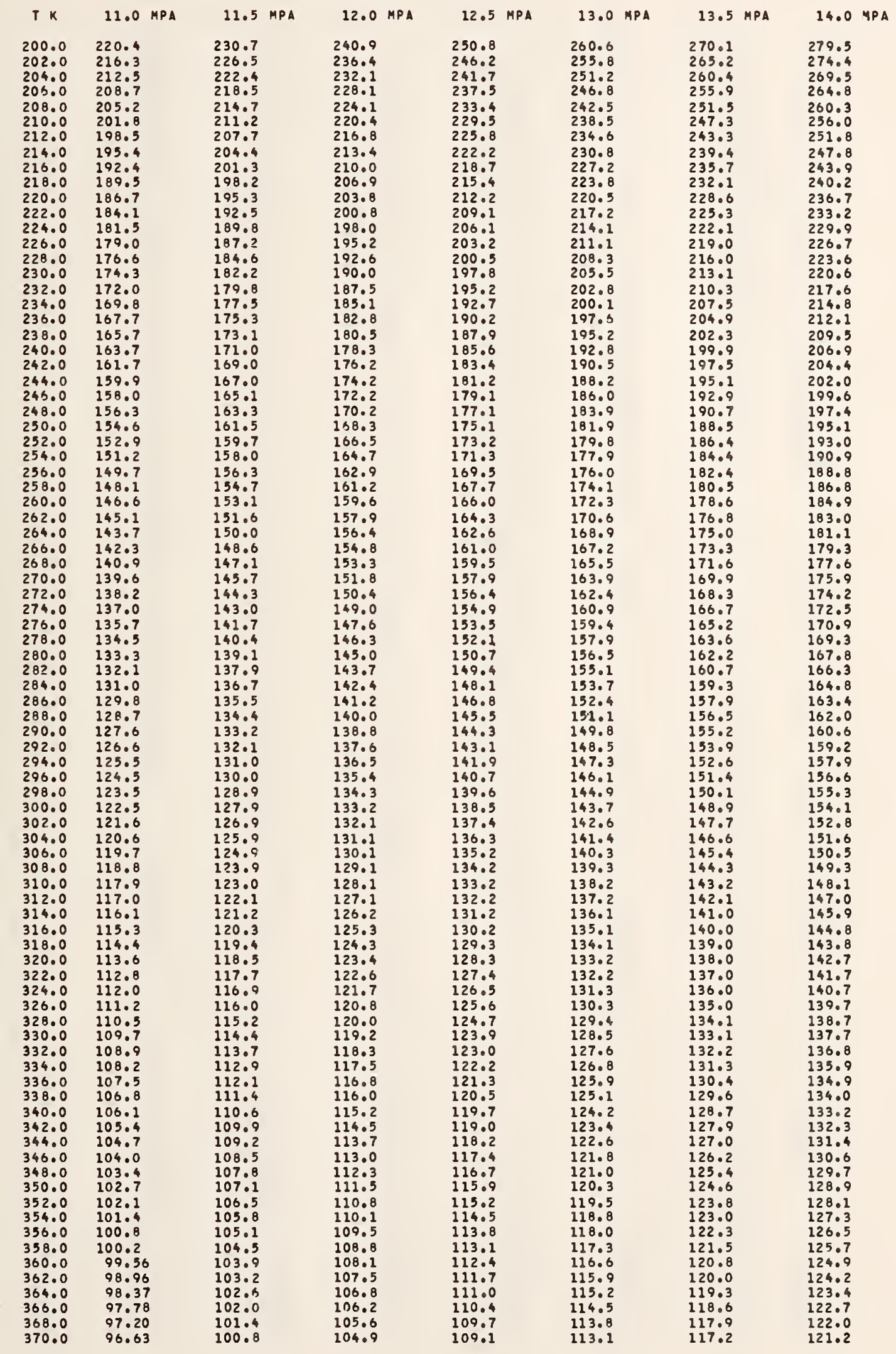


NITROGEN OENSITY (KG/M3).

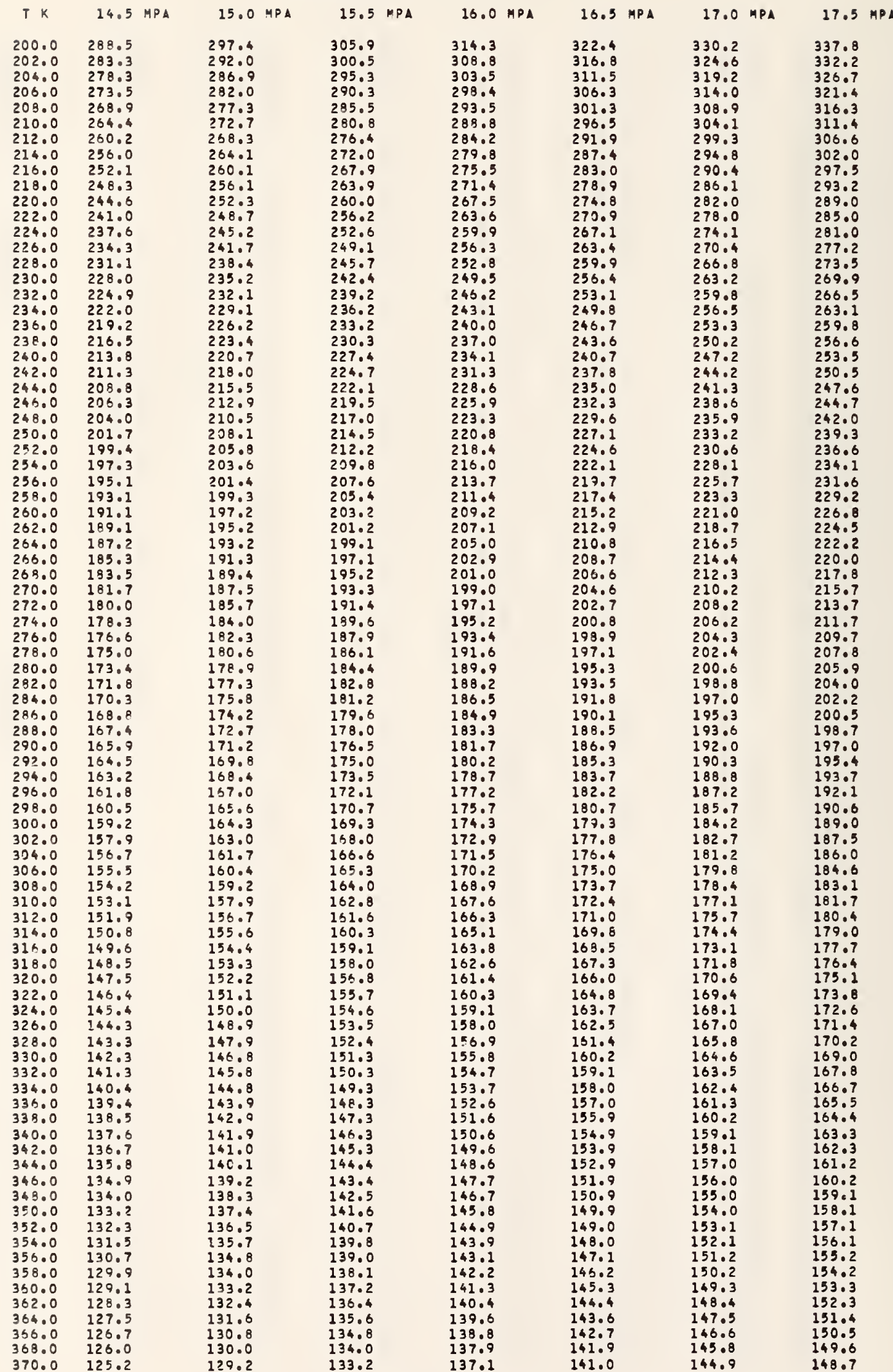


NITROGEN OENSITY (KG/M3).

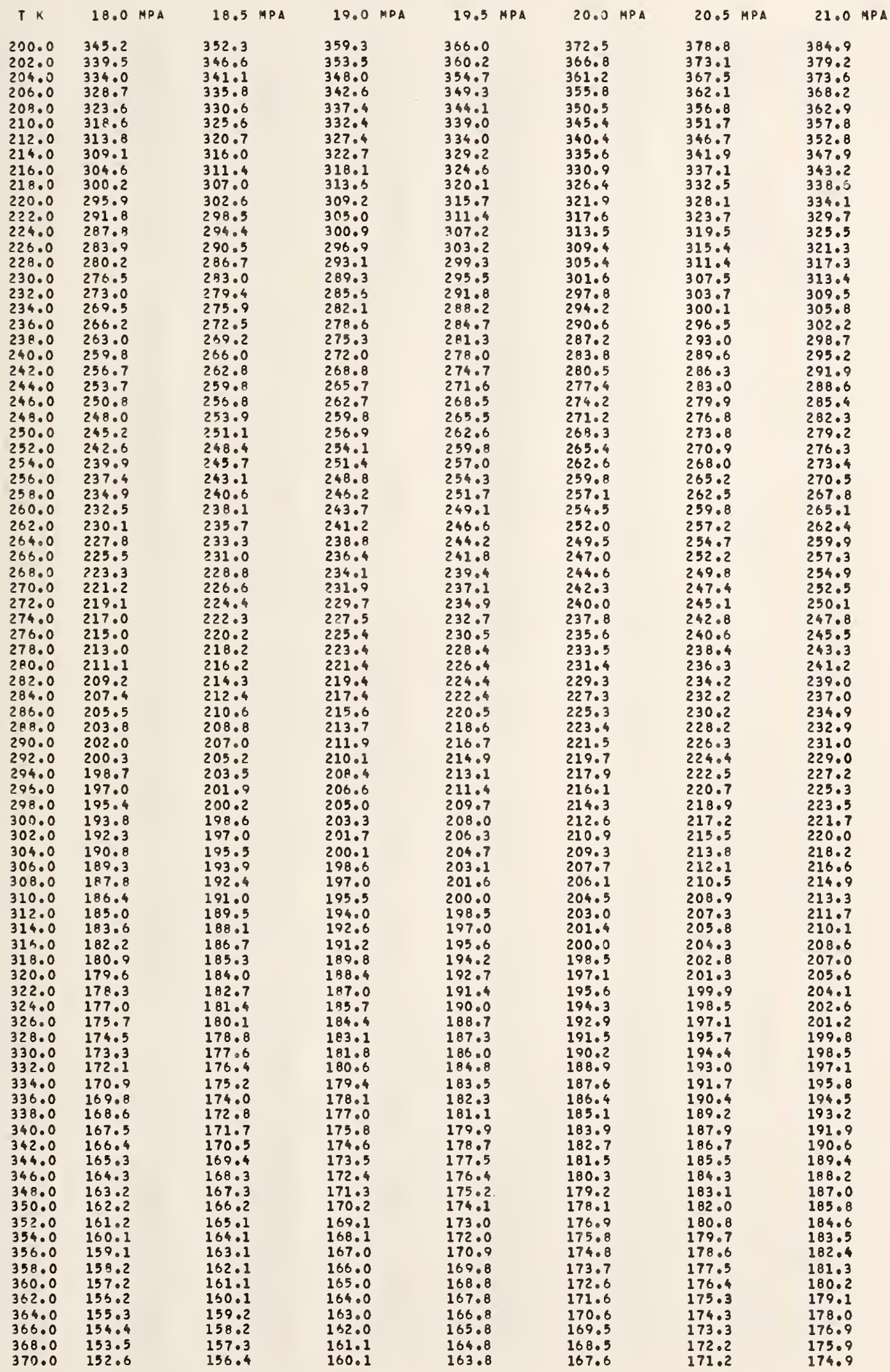


NITROGEN OENSITY (KG/M3).

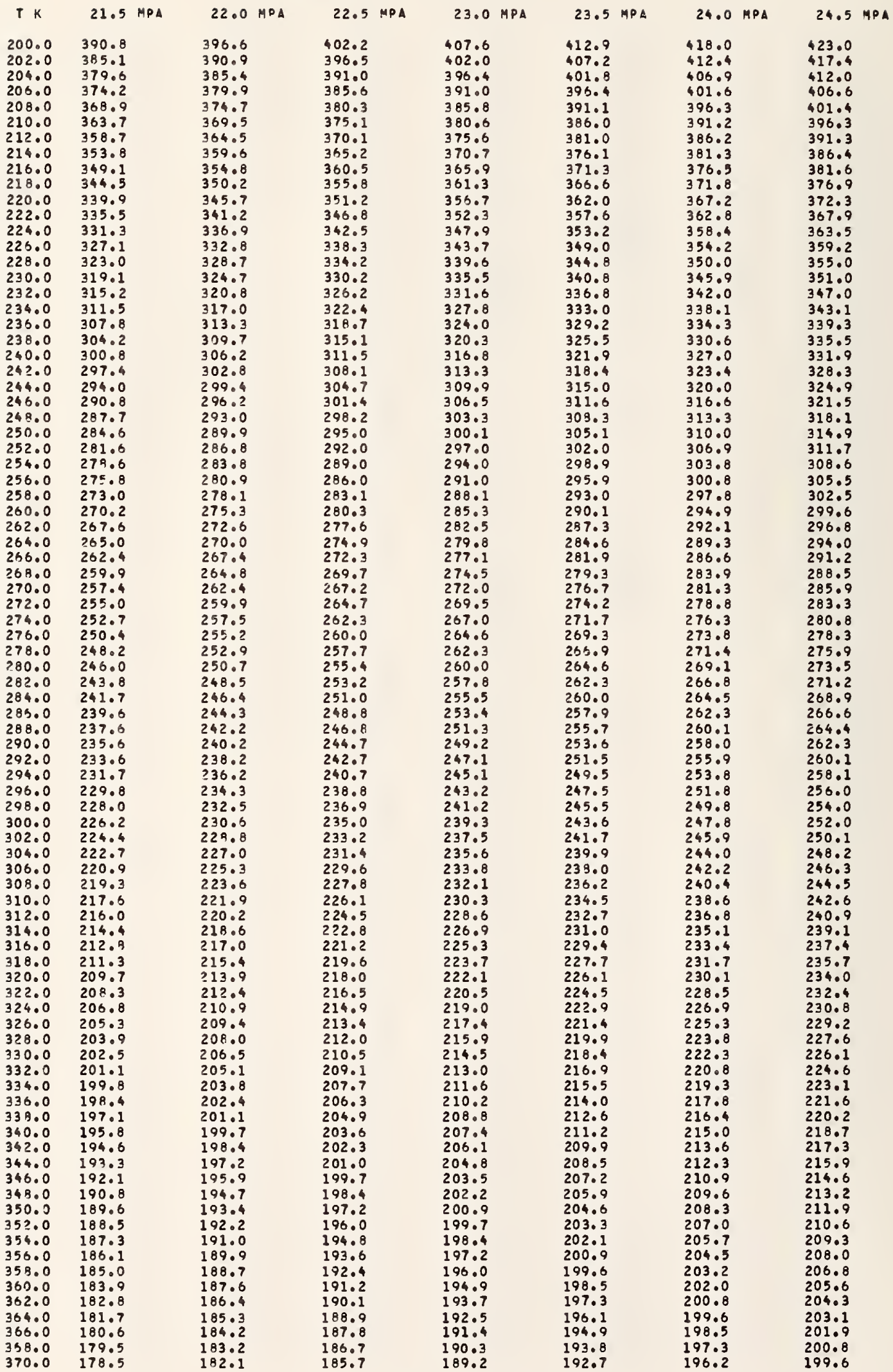


NITROGEN OENSITY (KG/M3).

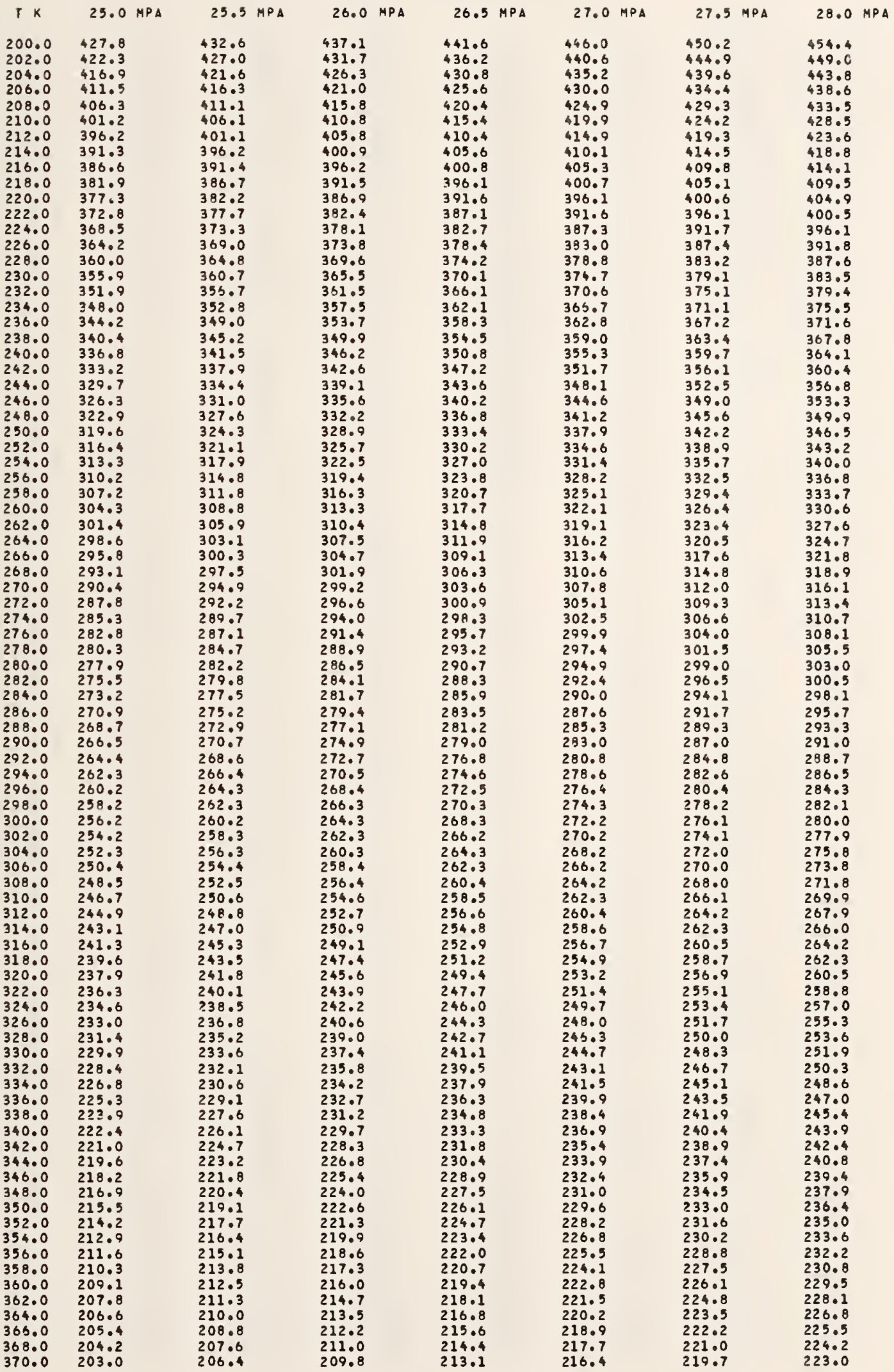


NITROGEN OENSITY (KG/M3).

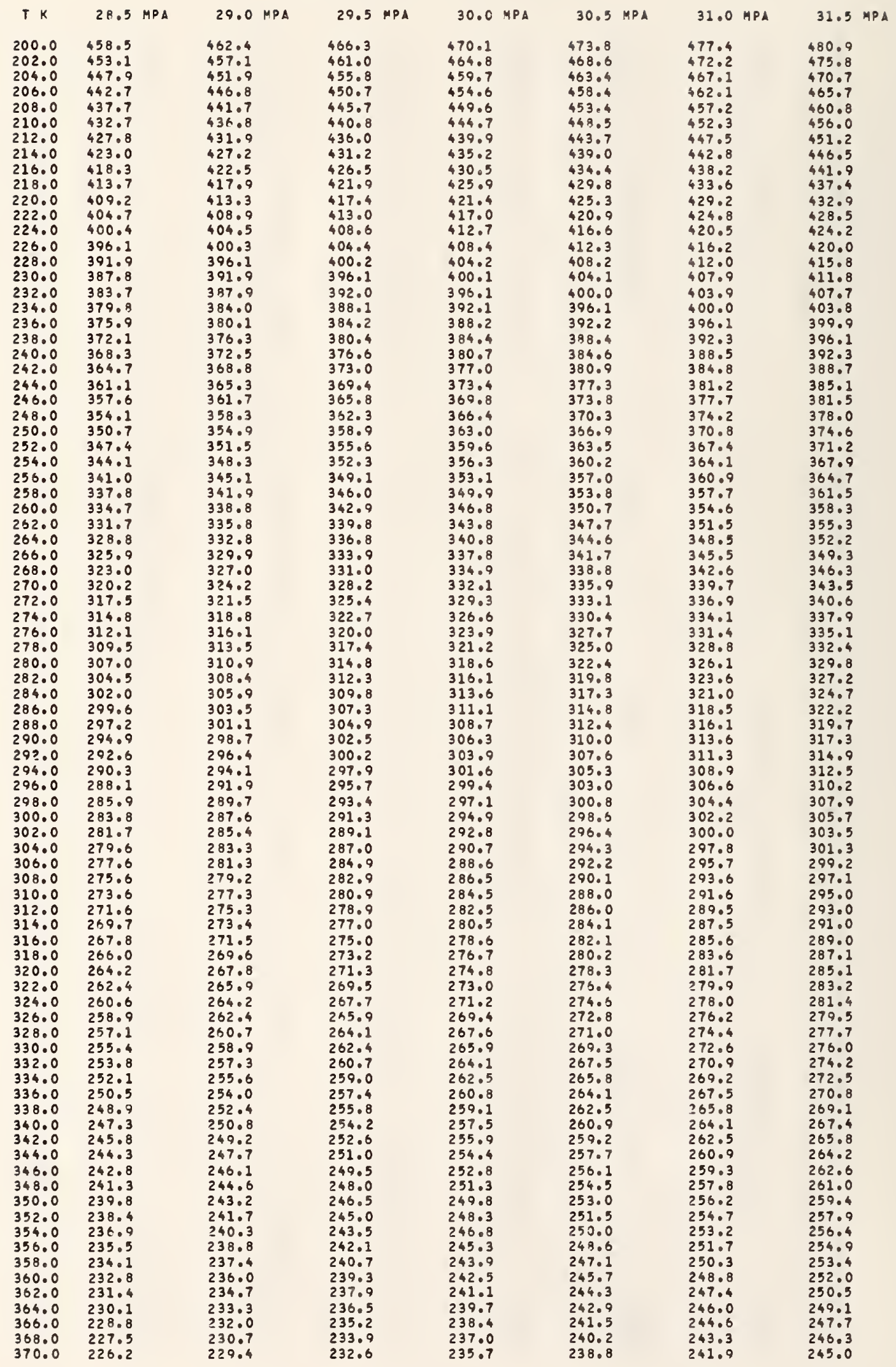


NITROGEN DENSITY (KG/H3).

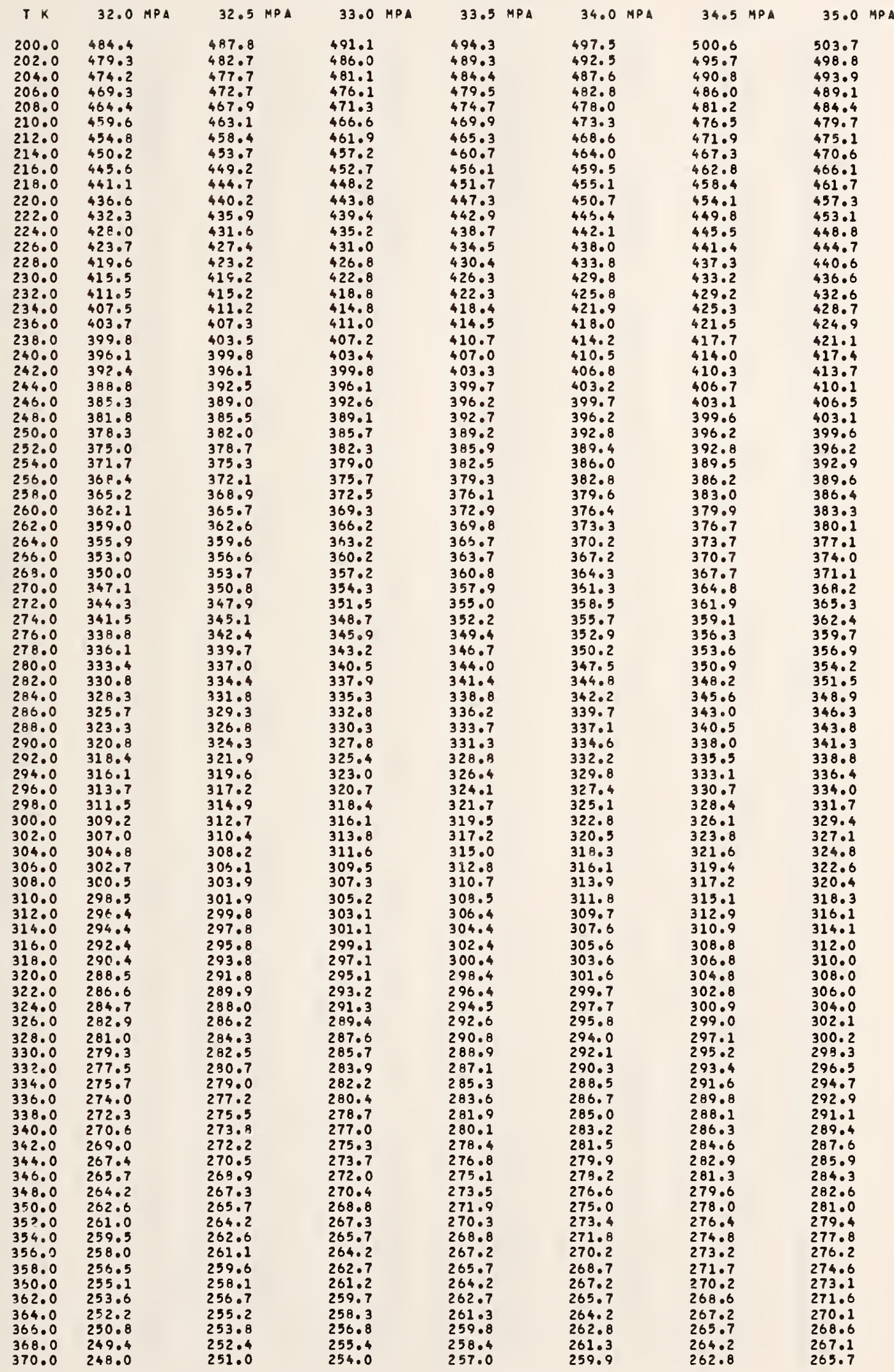


NITROGEN DENSITY (KG/H3).

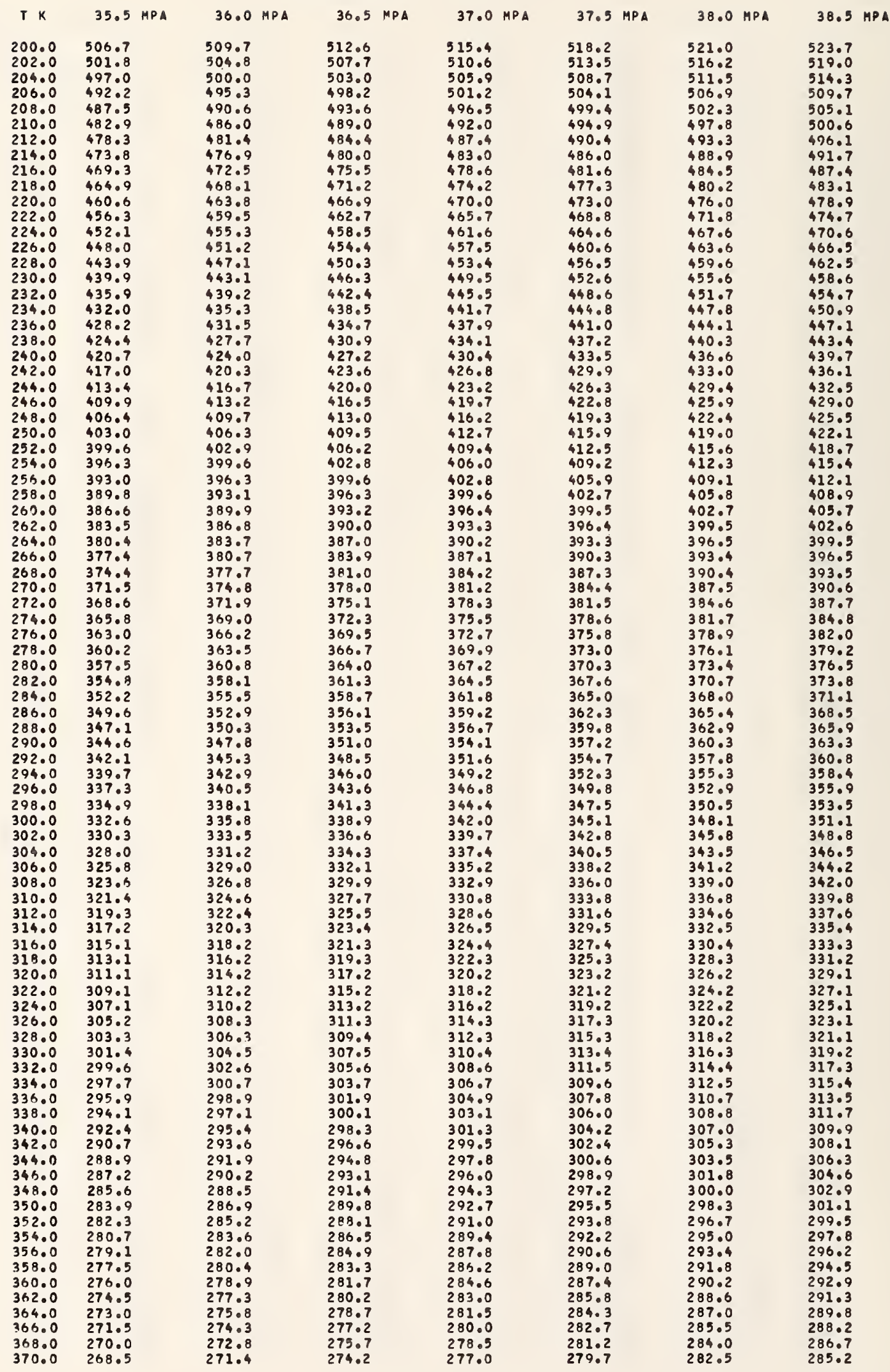


NITROGEN DENSITY (KG/M3).

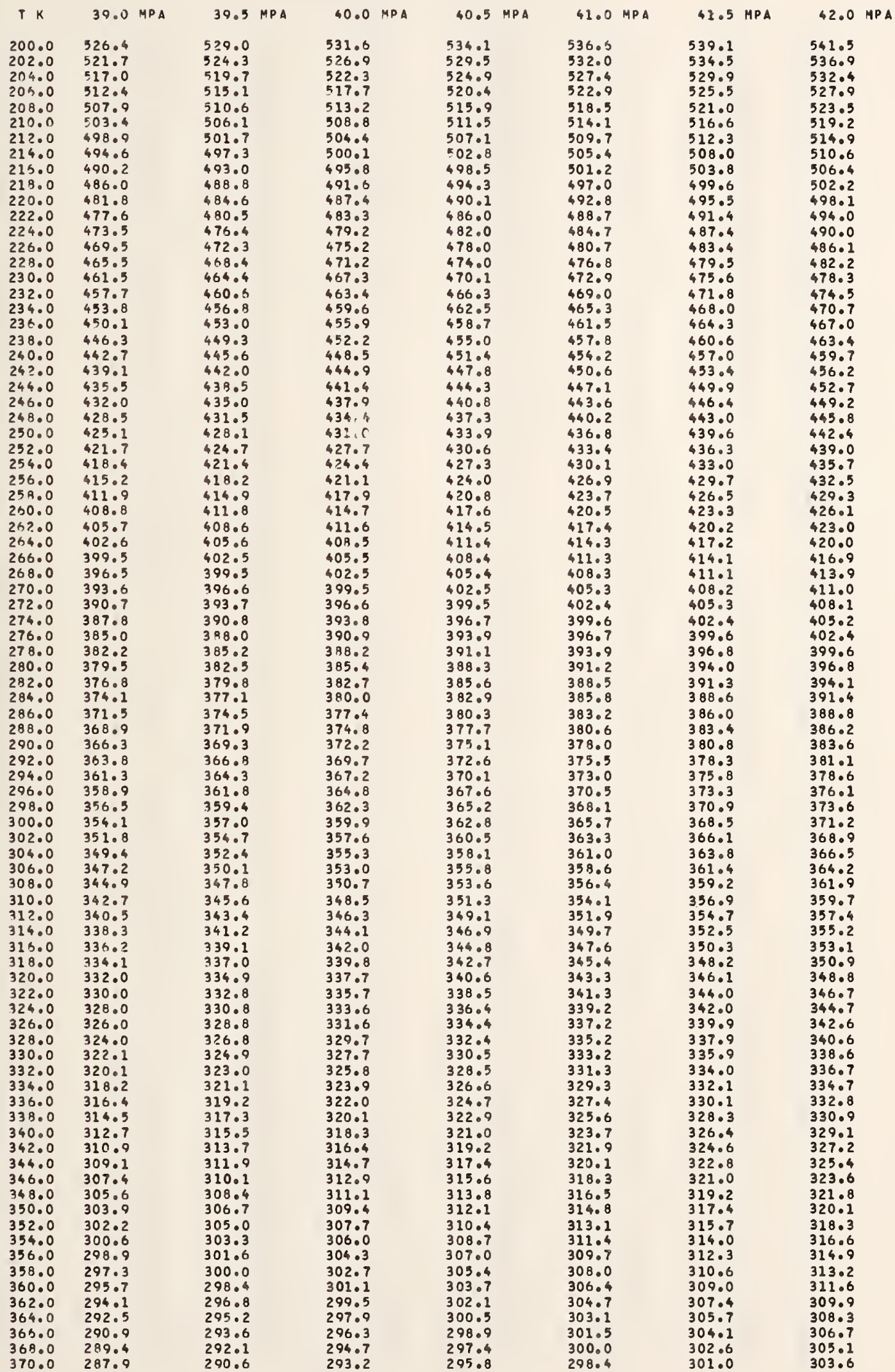


NITROGEN OENSITY (KG/M3).

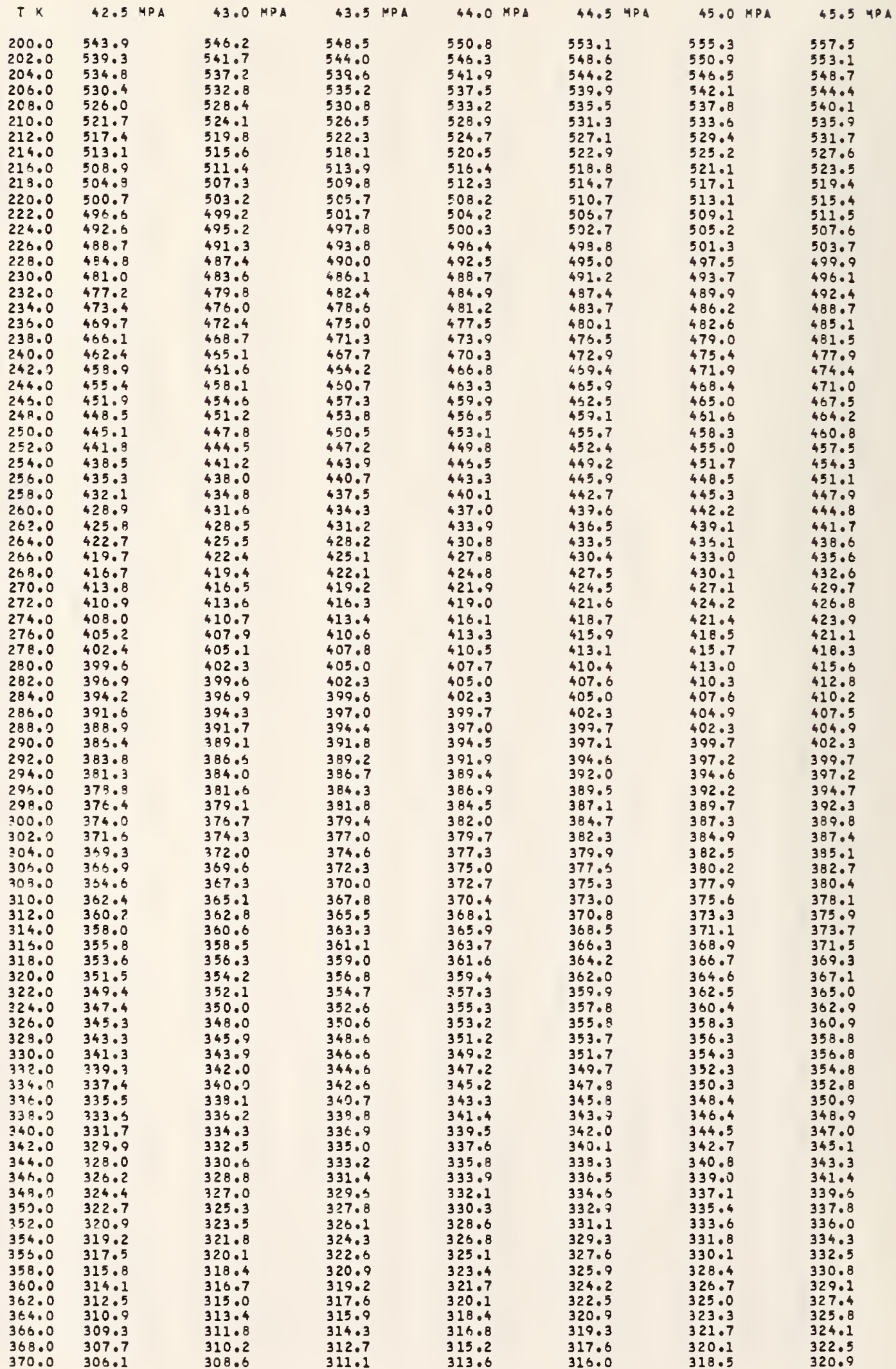


NITROGEN OENSITY (KG/H3).

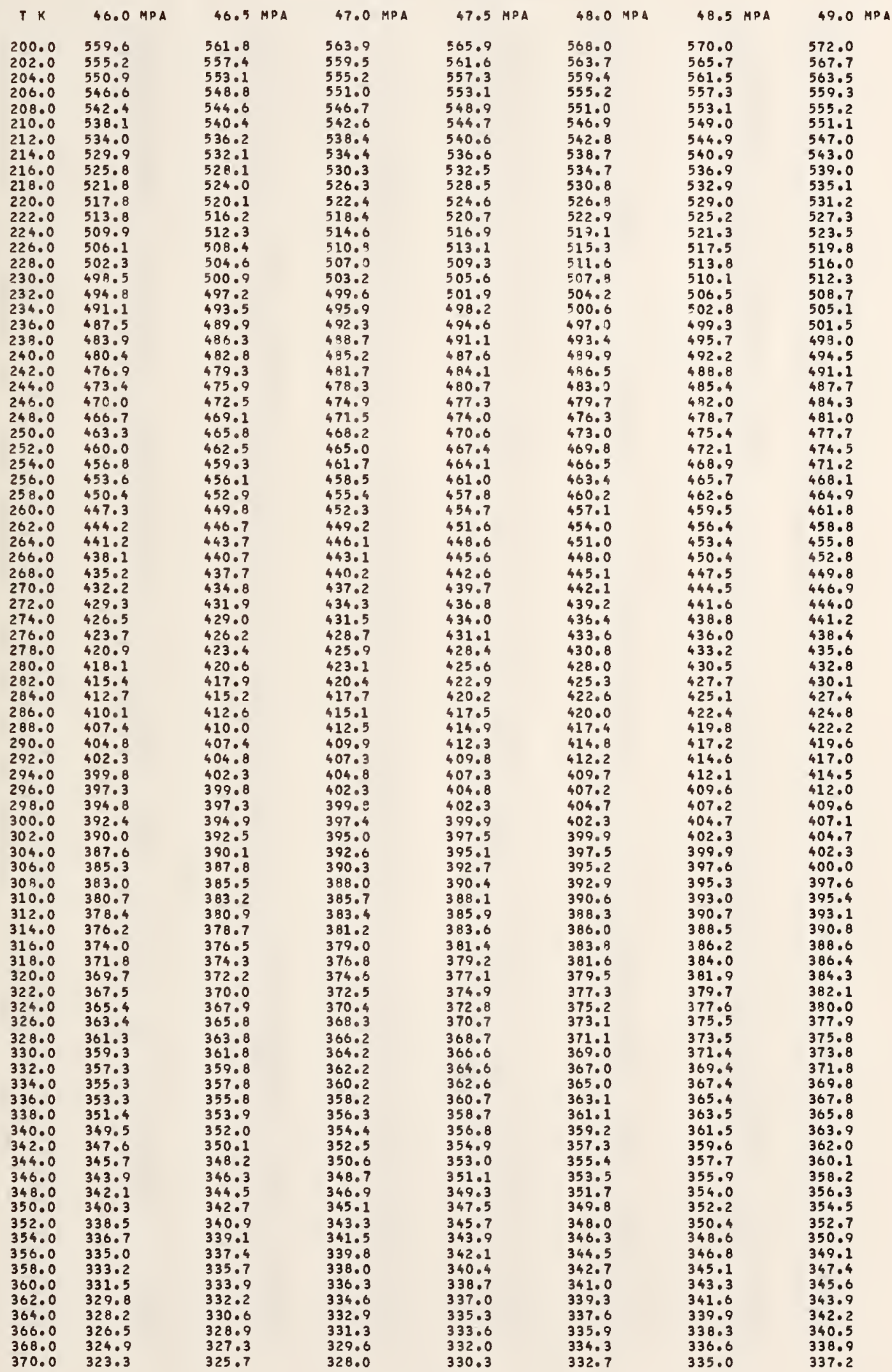


NITROGEN OENSITY (KG/H3).

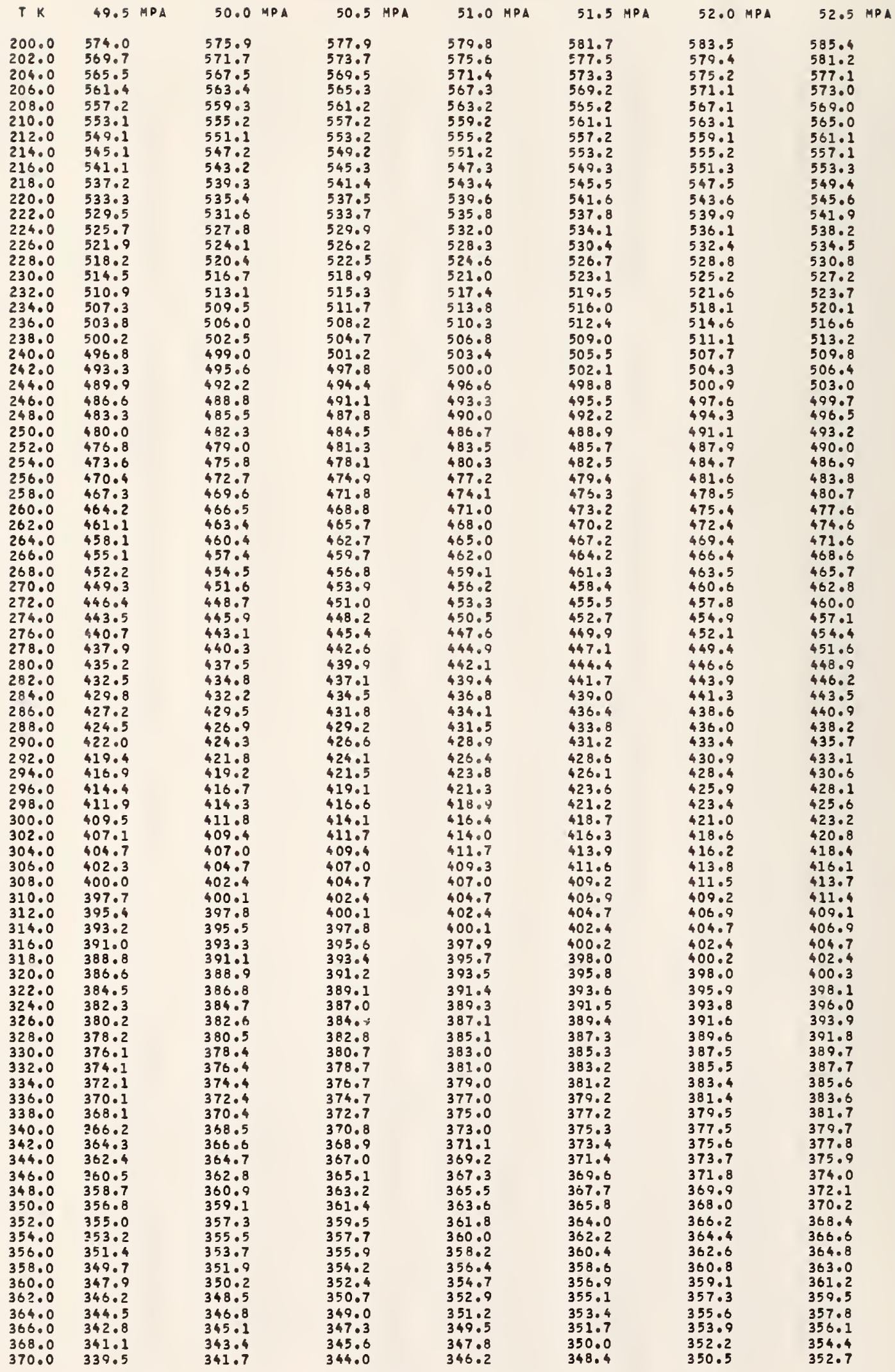


NITROGEN OENSITY (KG/M3).

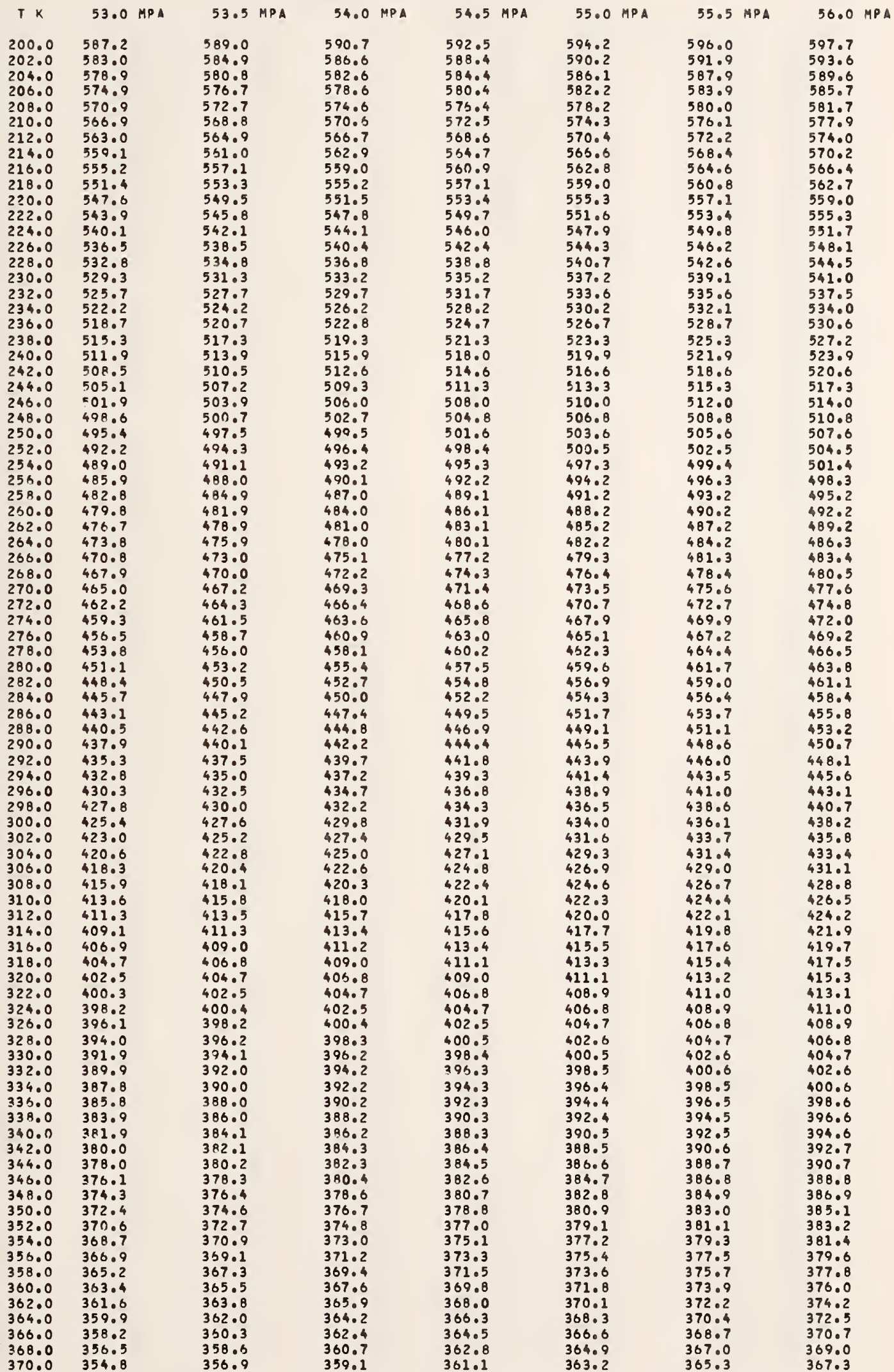


NITROGEN OENSITY (KG/M3).

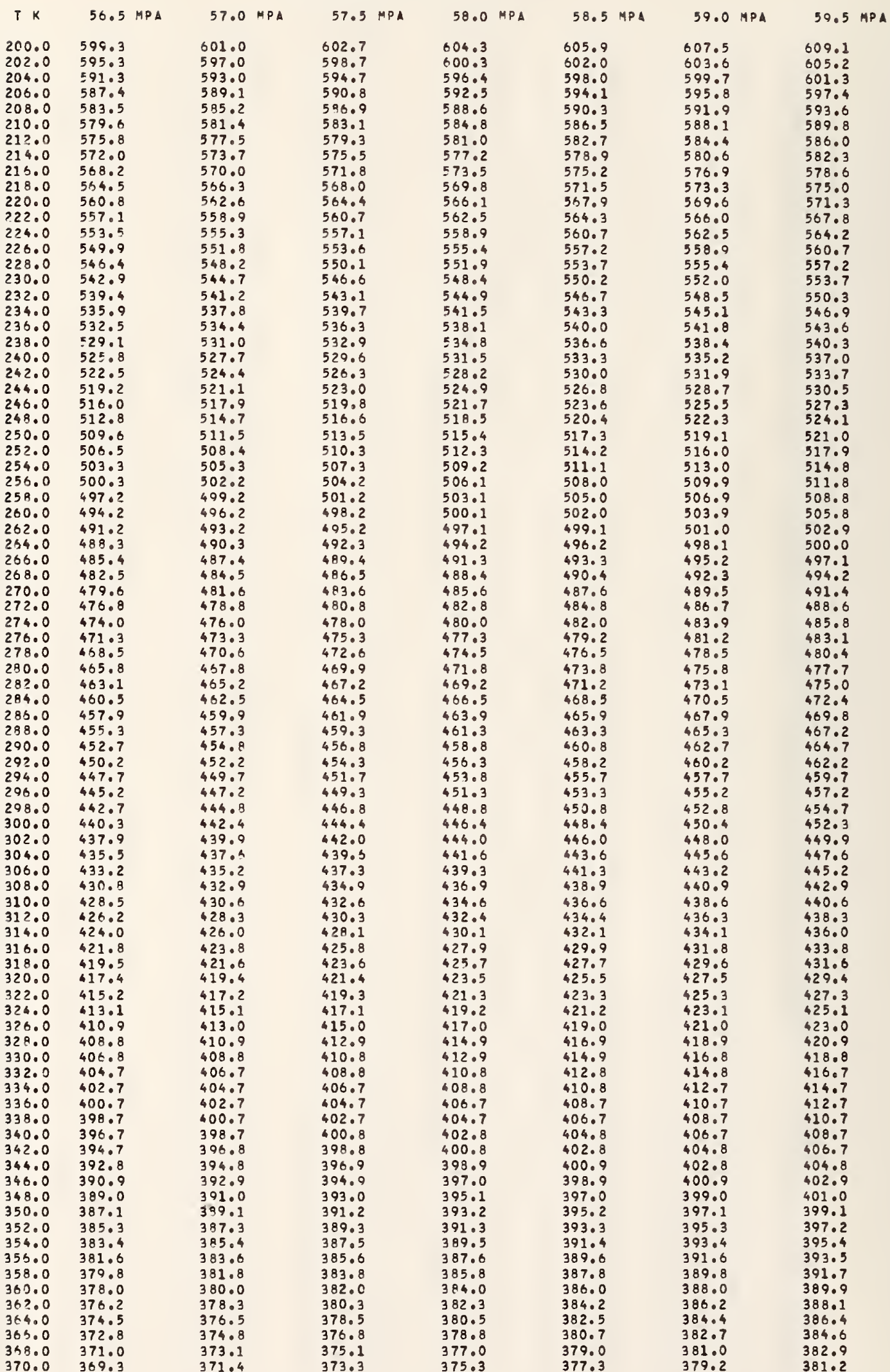


NITROGEN DENSITY (KG/M3).

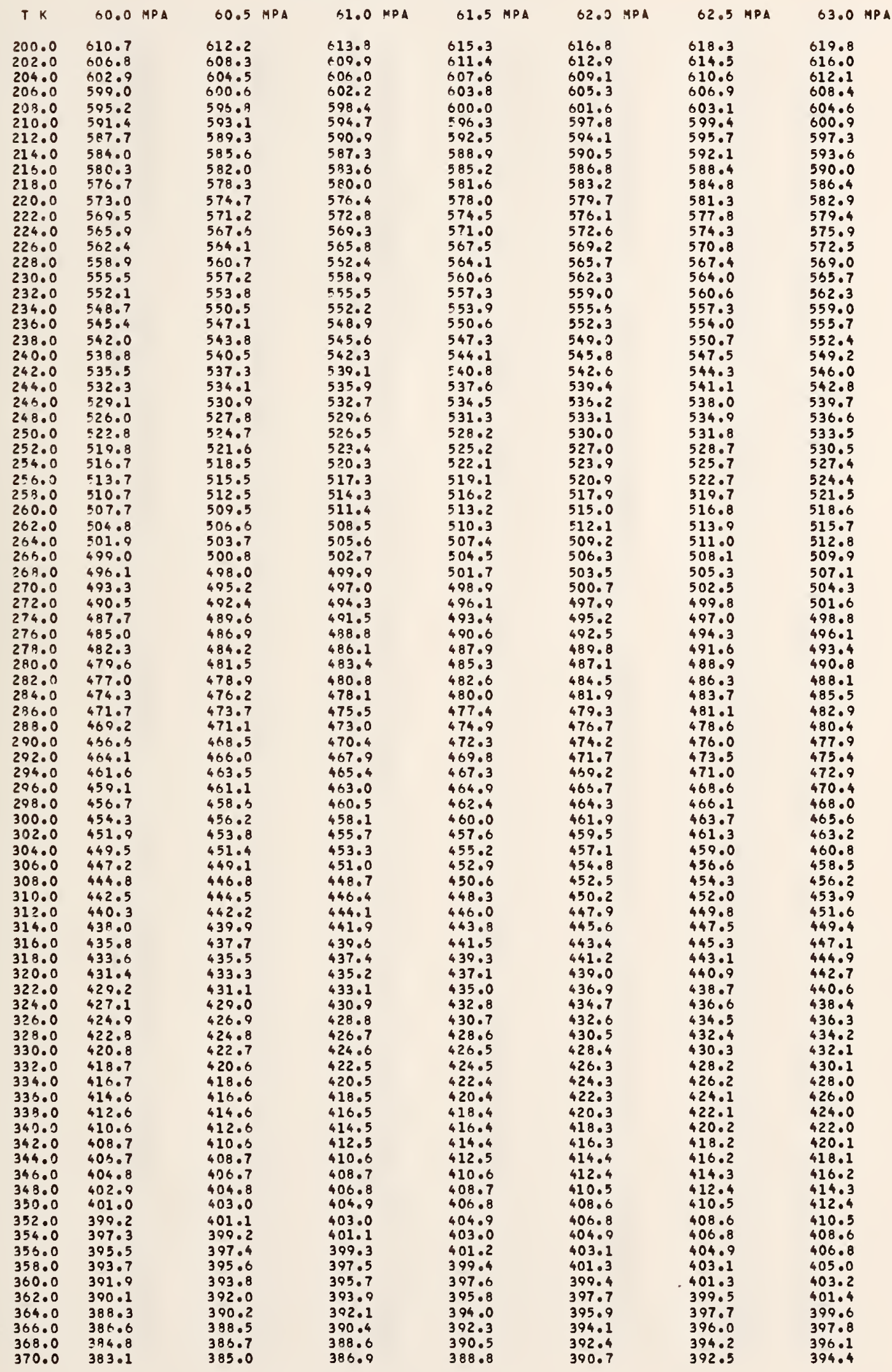


NITROGEN DENSITY (KG/M3).

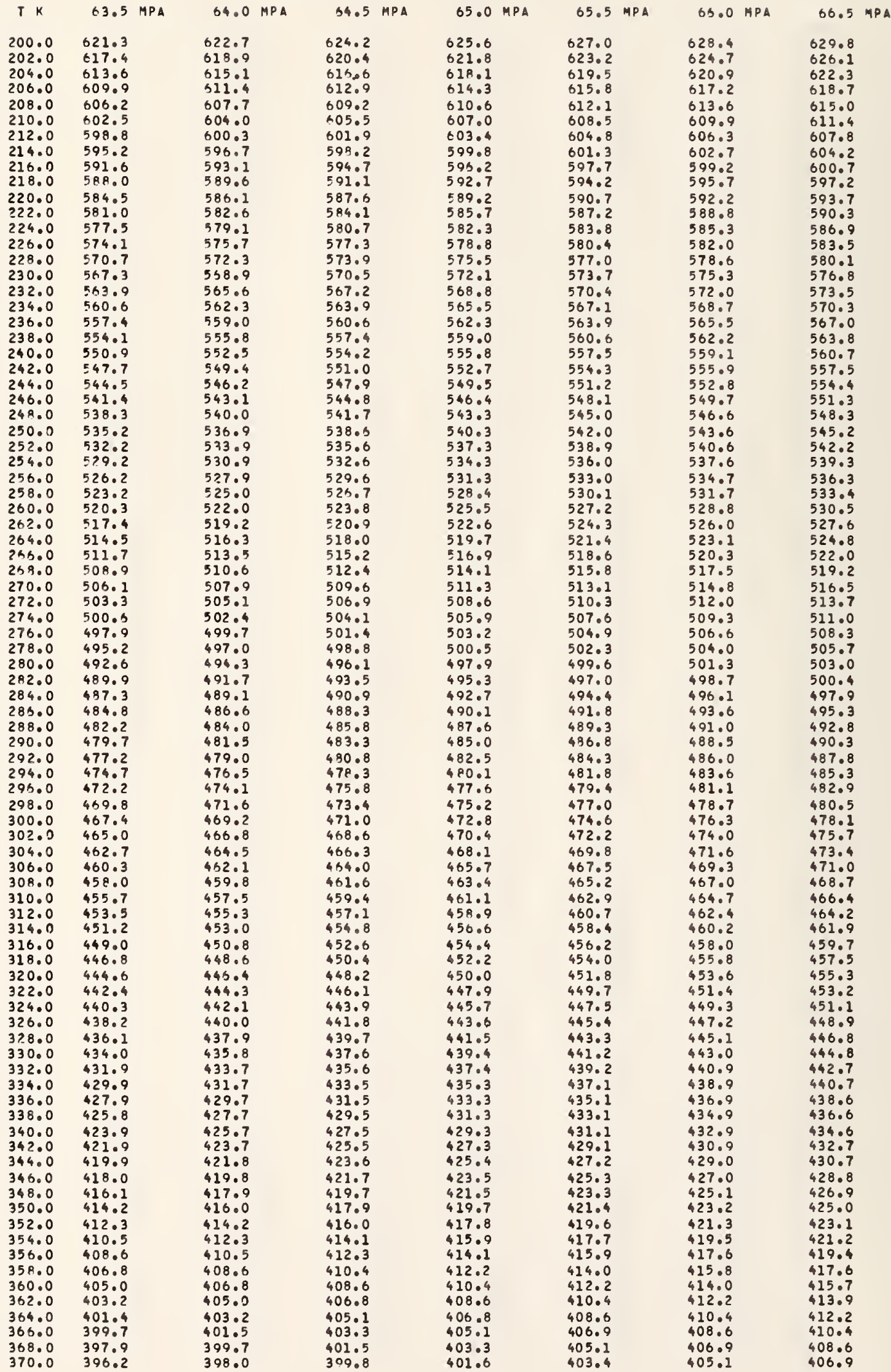


NITROGEN DENSITY (KG/M3).

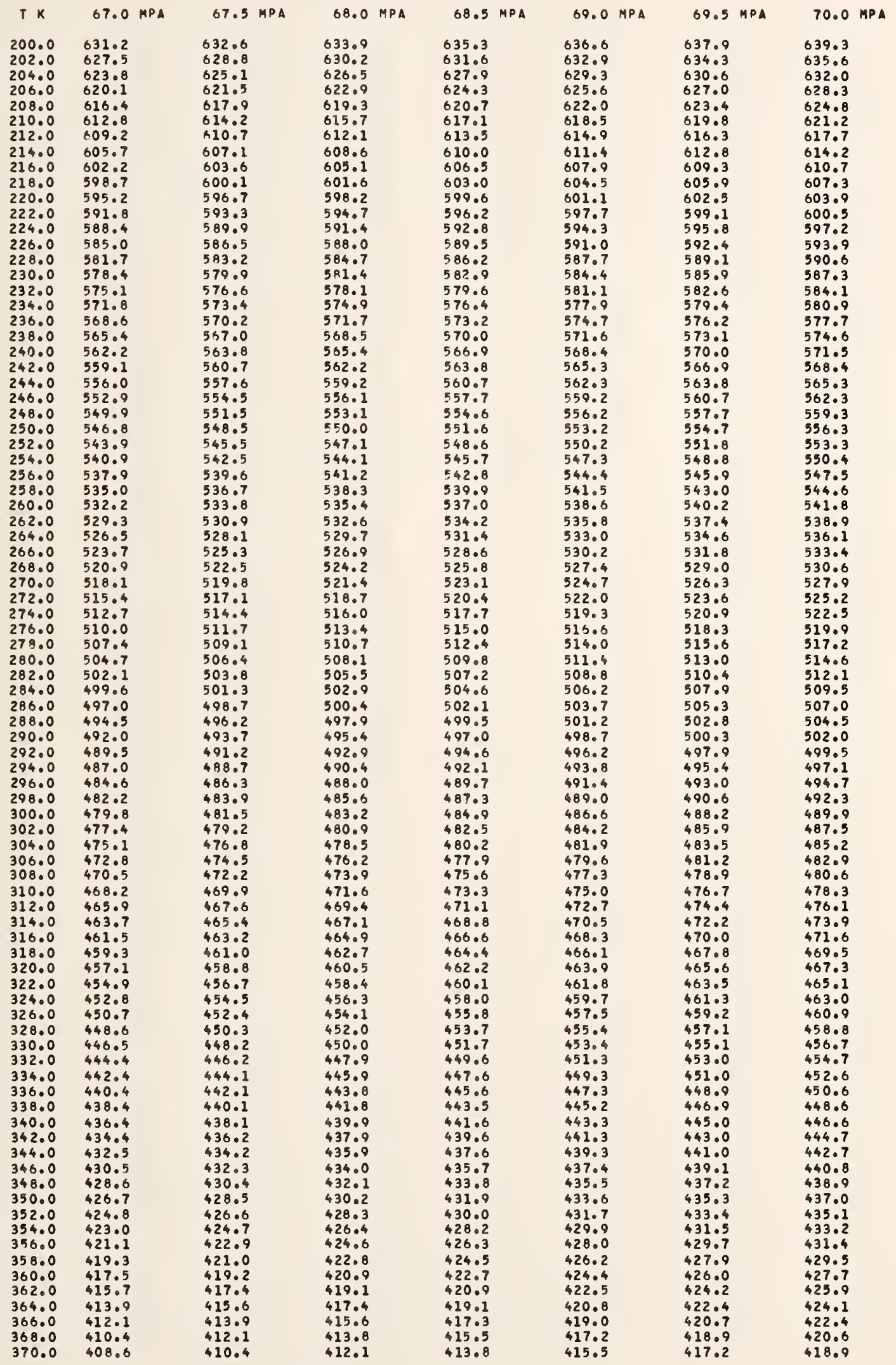




$$
9.4
$$

Helium Density $\left(\mathrm{kg} / \mathrm{m}^{3}\right)$ 
HELIUH OENSITY (KG/M3).

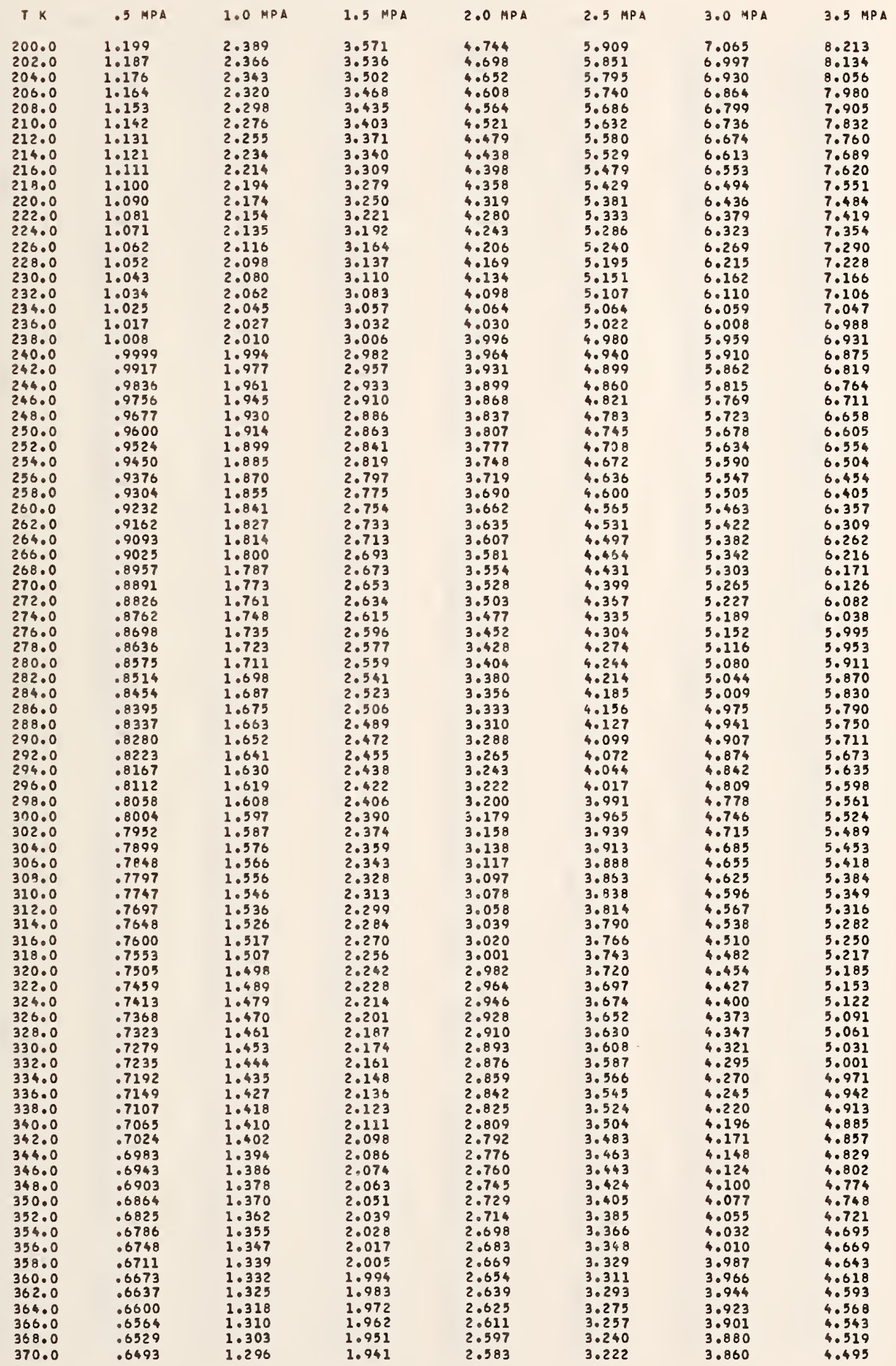


HELIUM OENSITY (KG/M3).

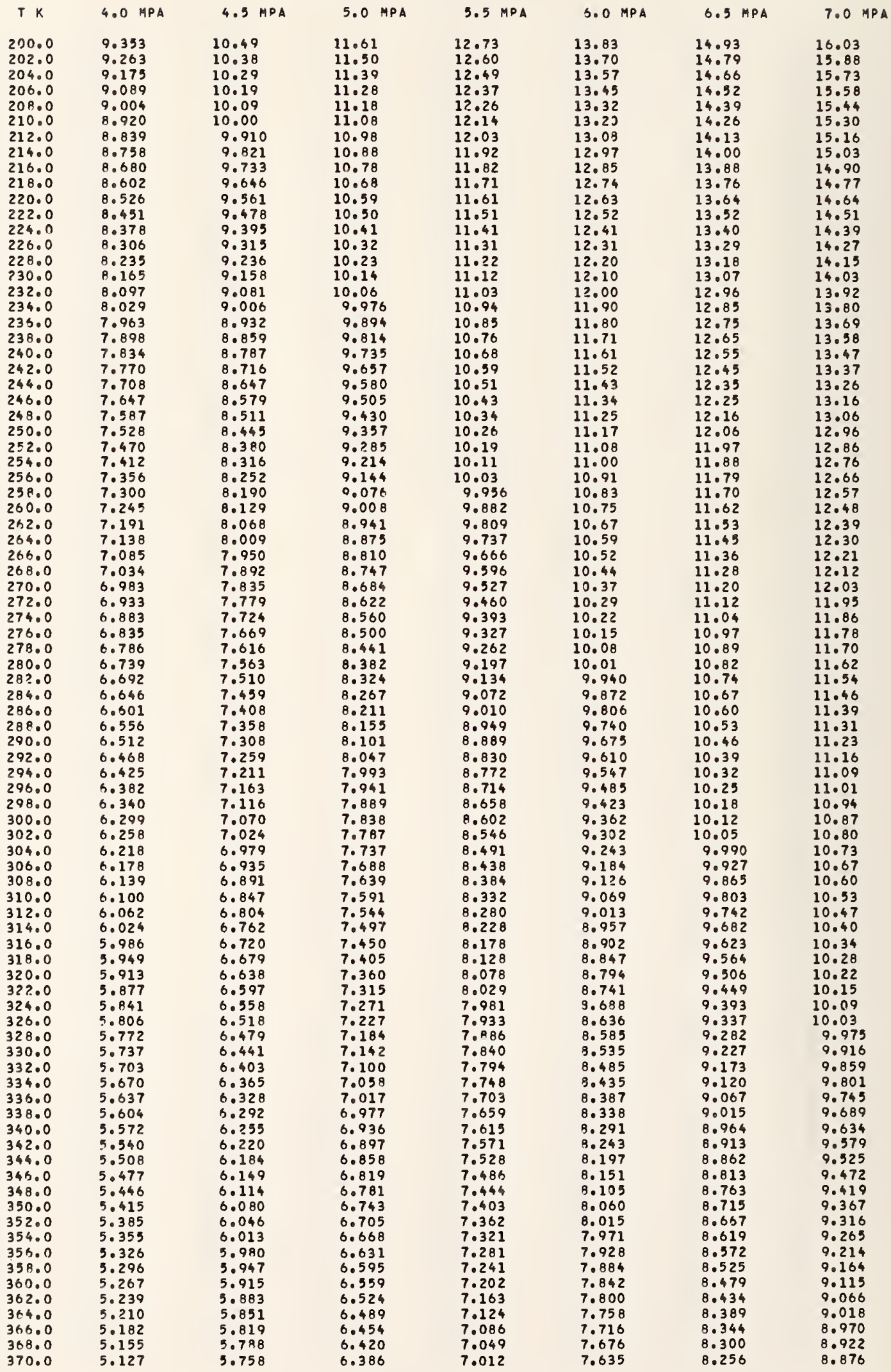


HELIUM OENSITY (KG/M3).

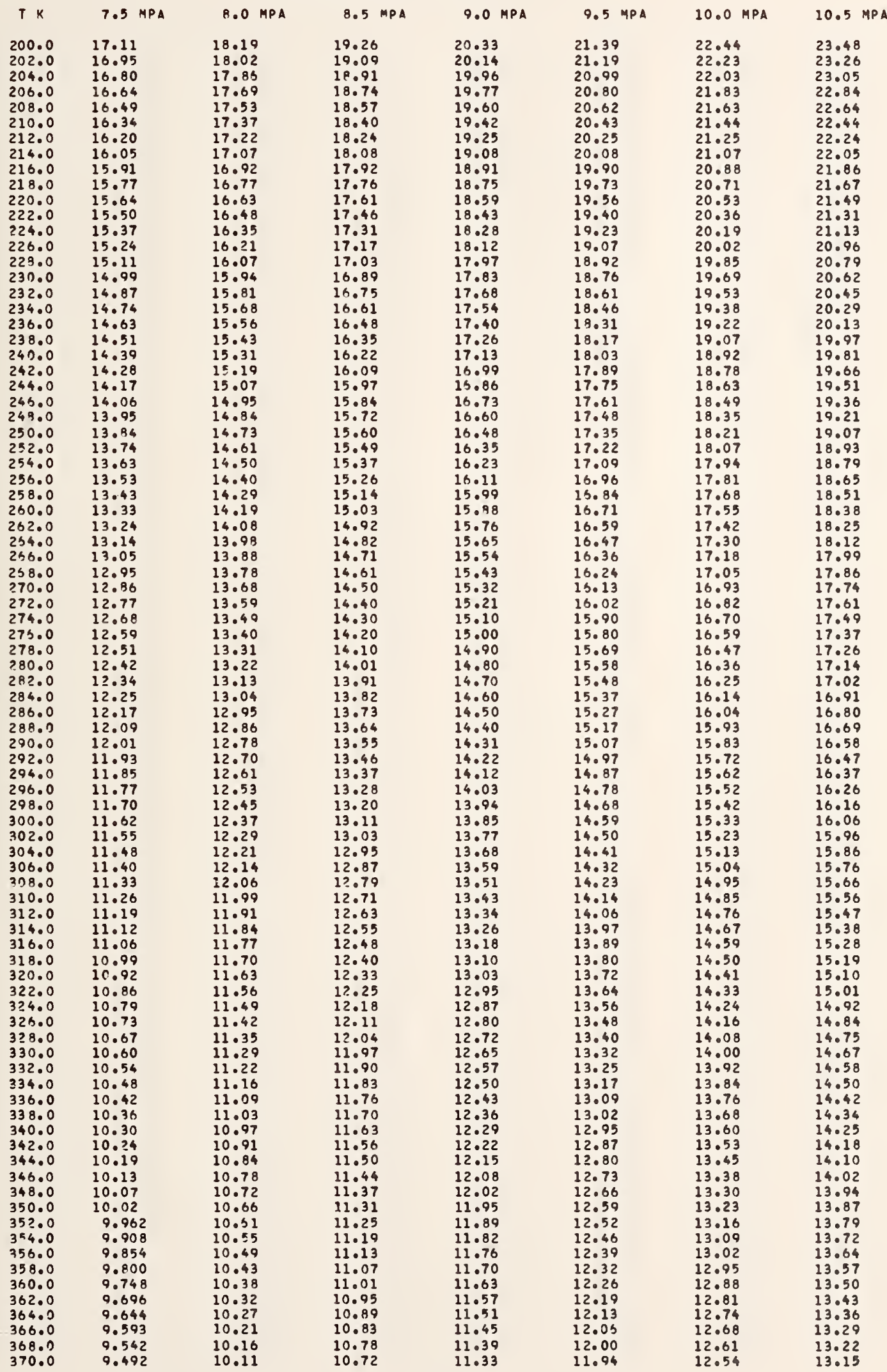


HELIUM DENSITY (KG/M3)

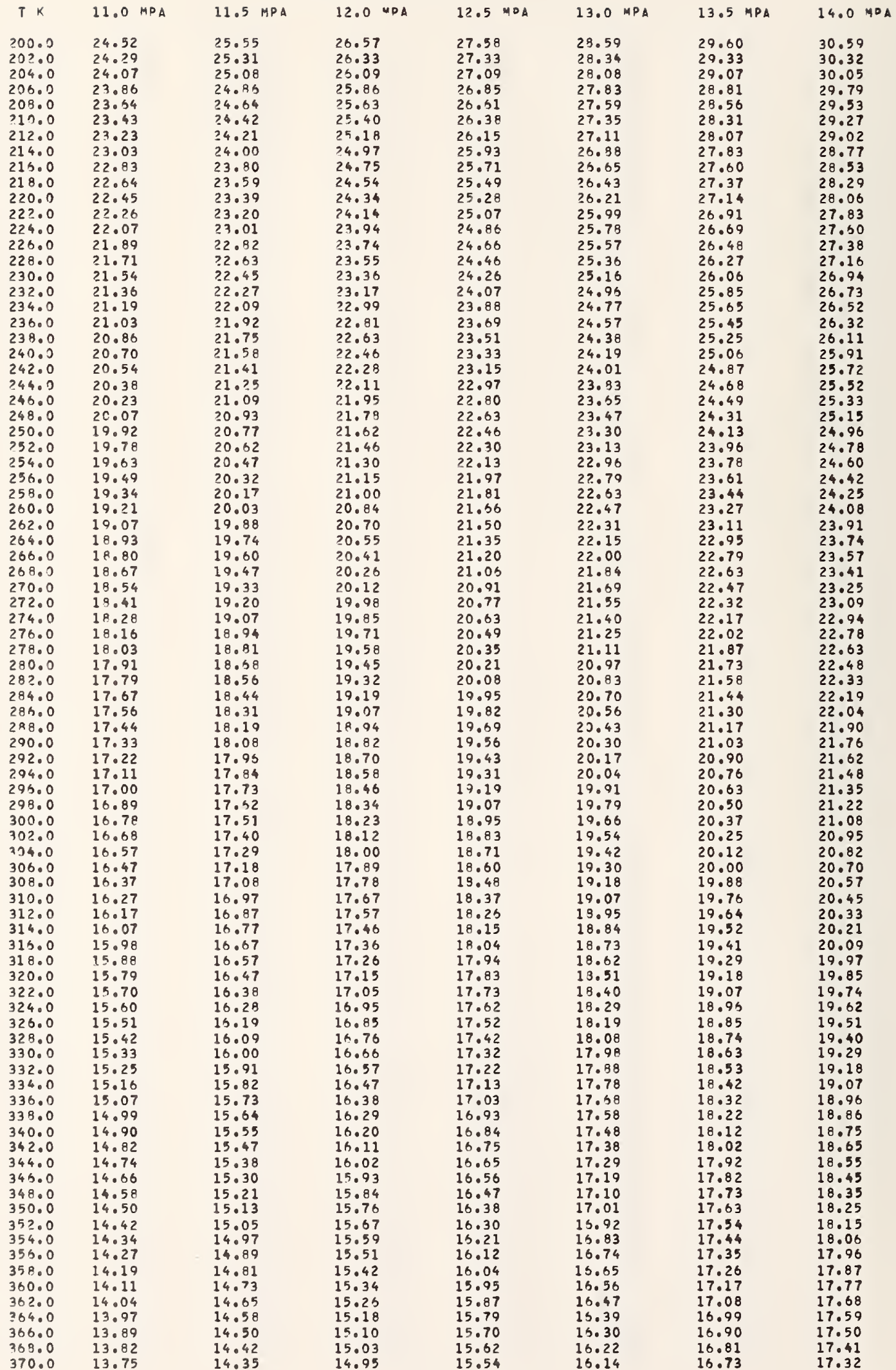


HELIUN DENSITY (KG/M3).

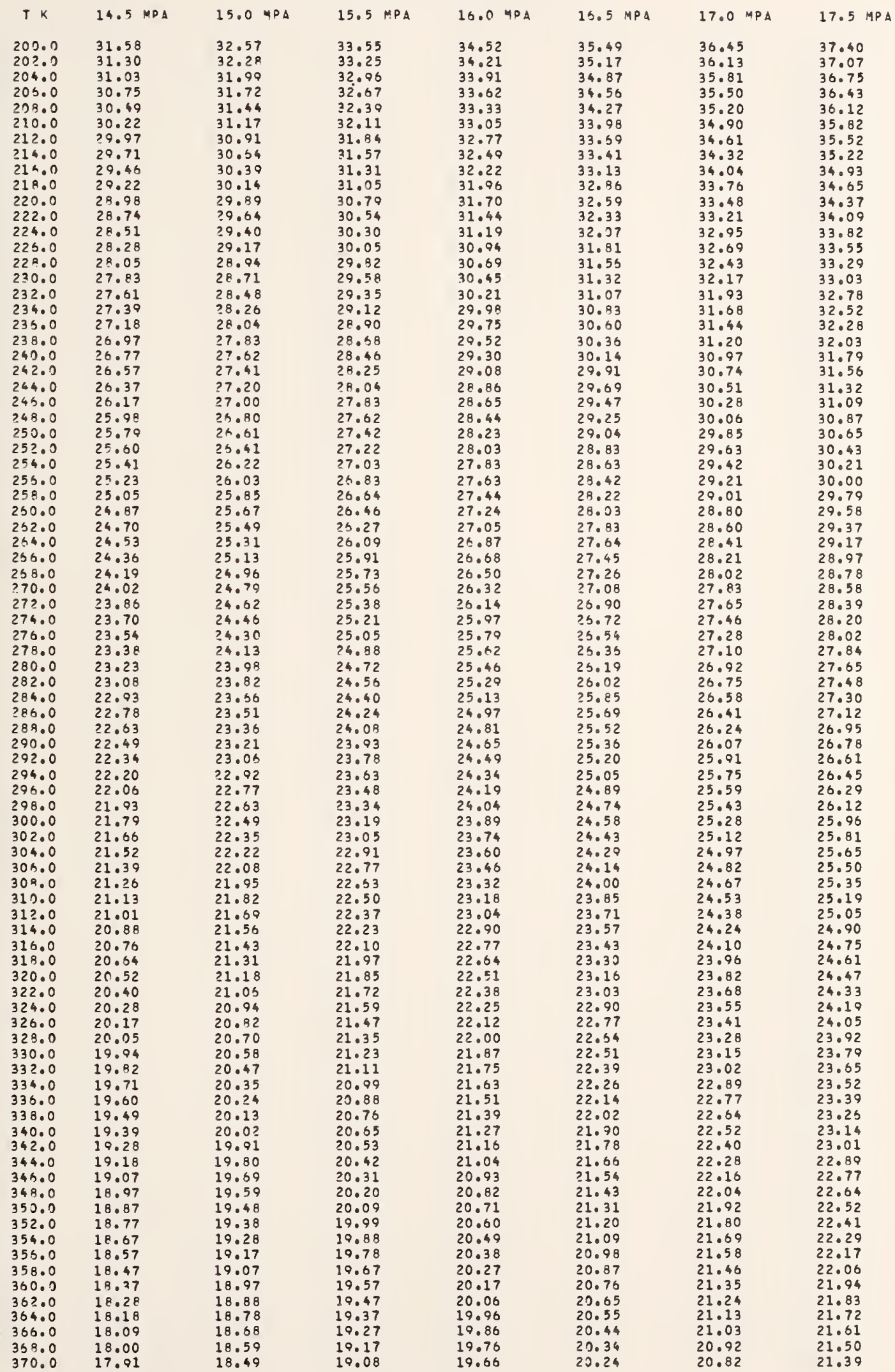


HELIUK OENSITY (KG/43).

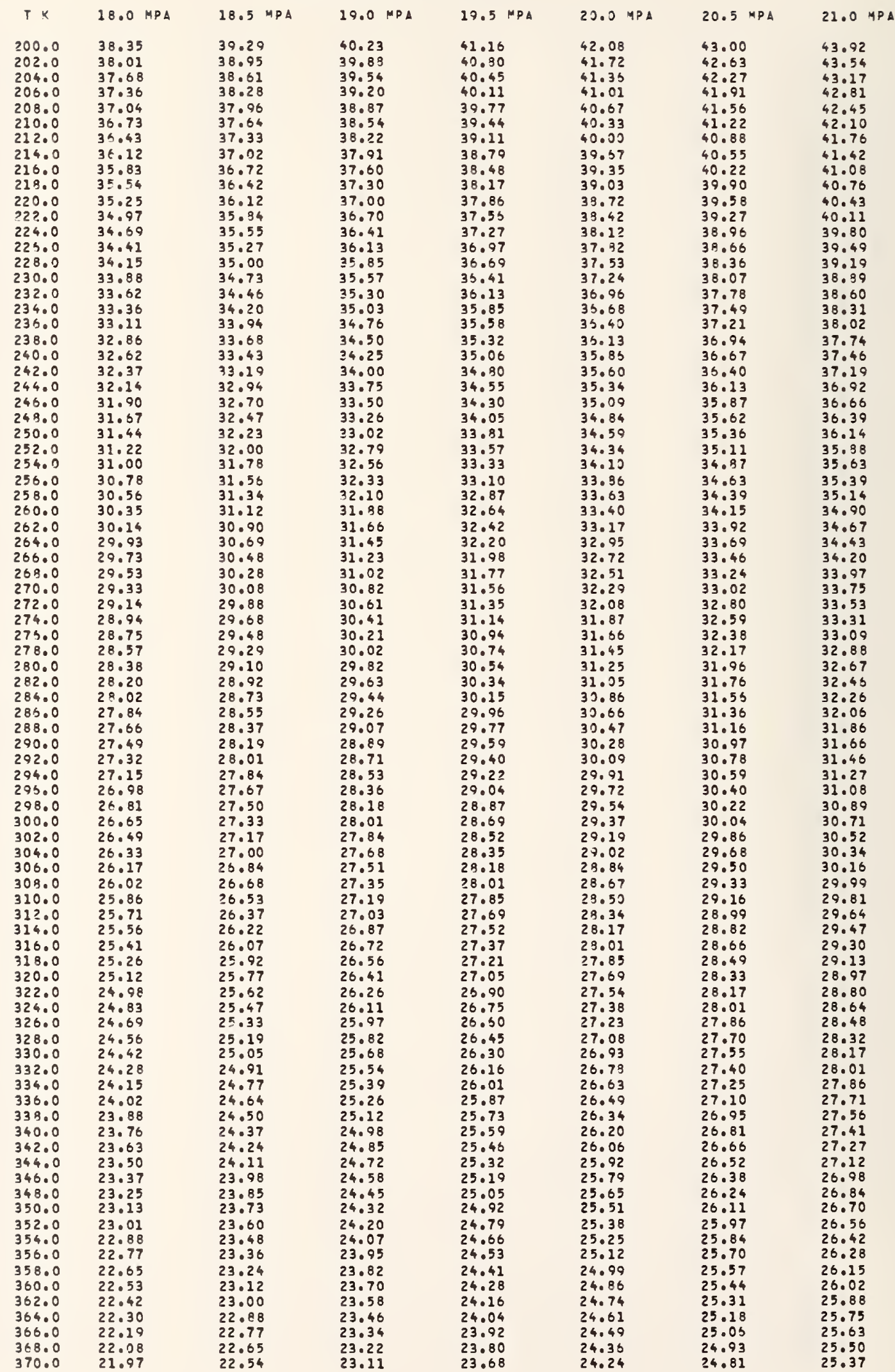


HELIUM DENSITY (KG/M3).

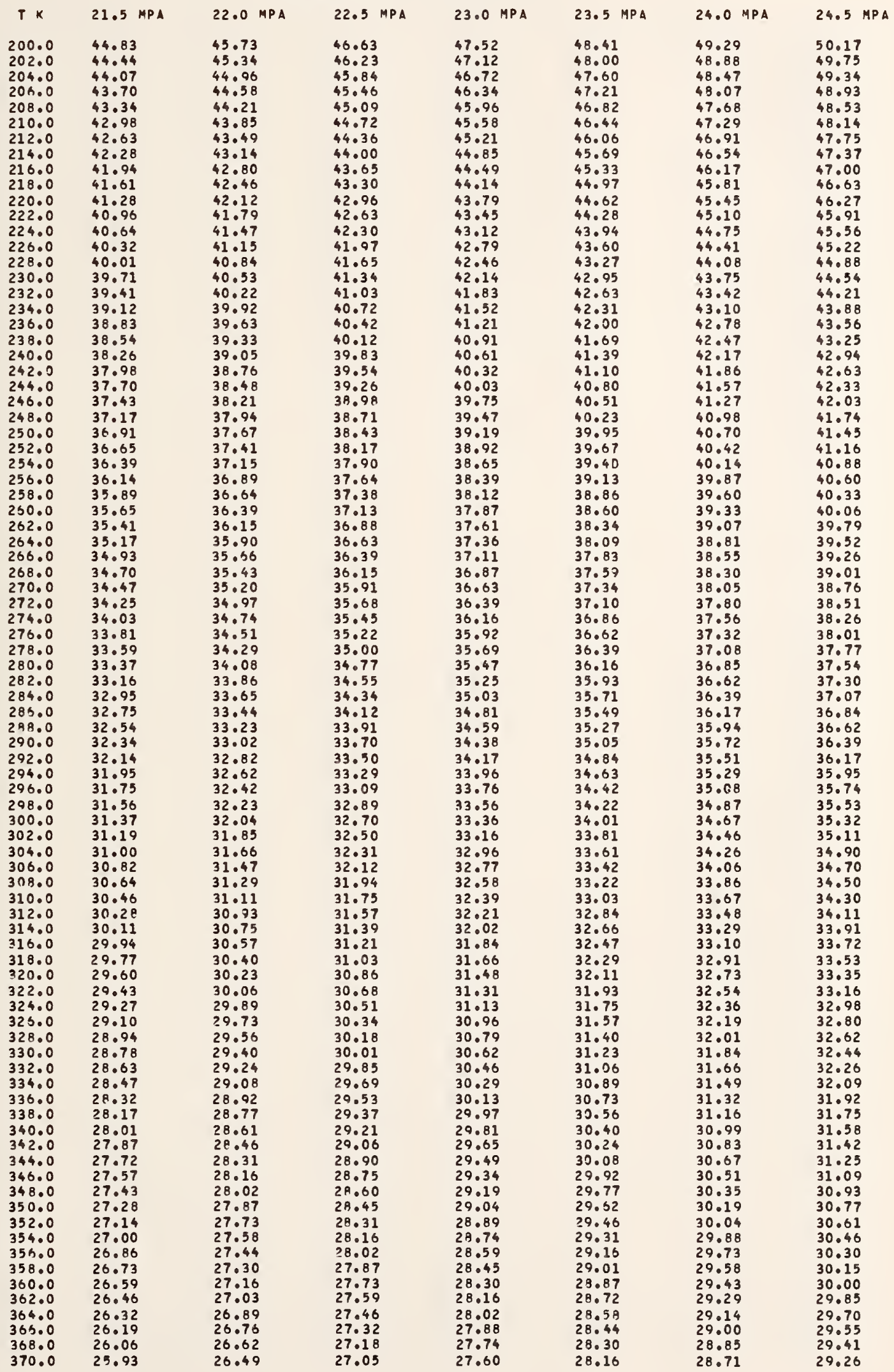


HELIUM OENSITY (KG/MB).

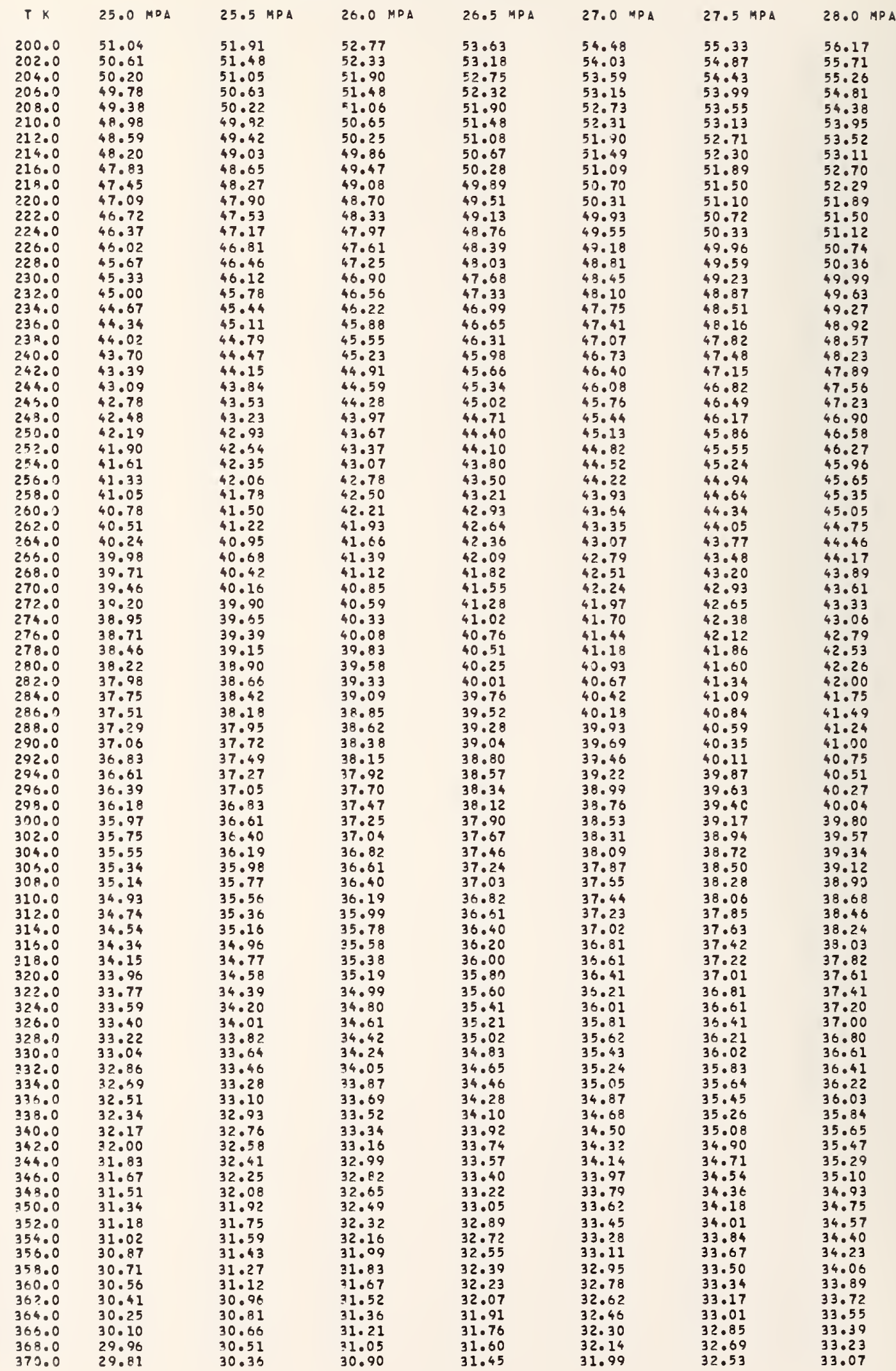


HELIUM DENSITY (KG/M3)。

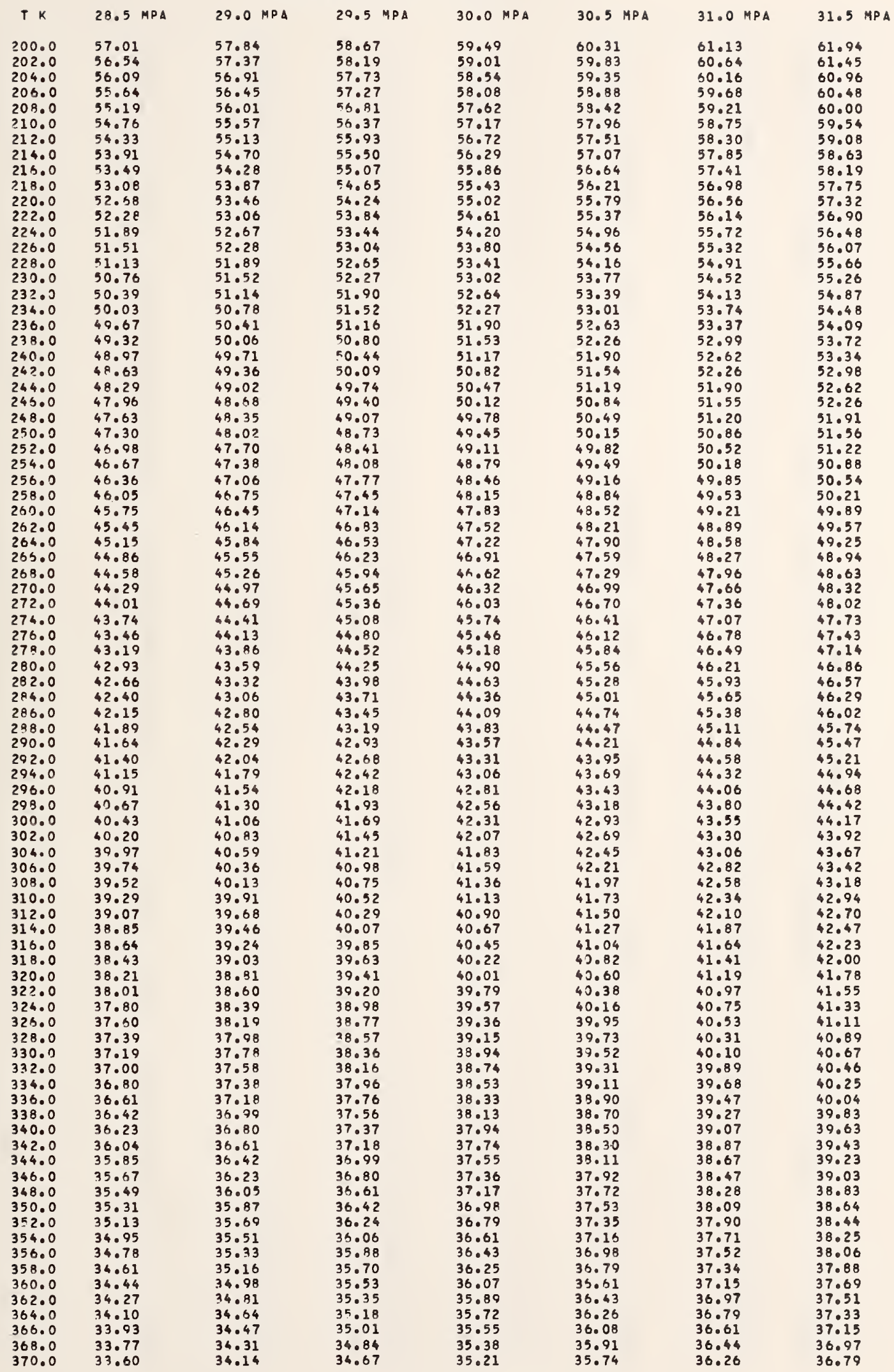


HELIUN DENSITY (KG/MB).

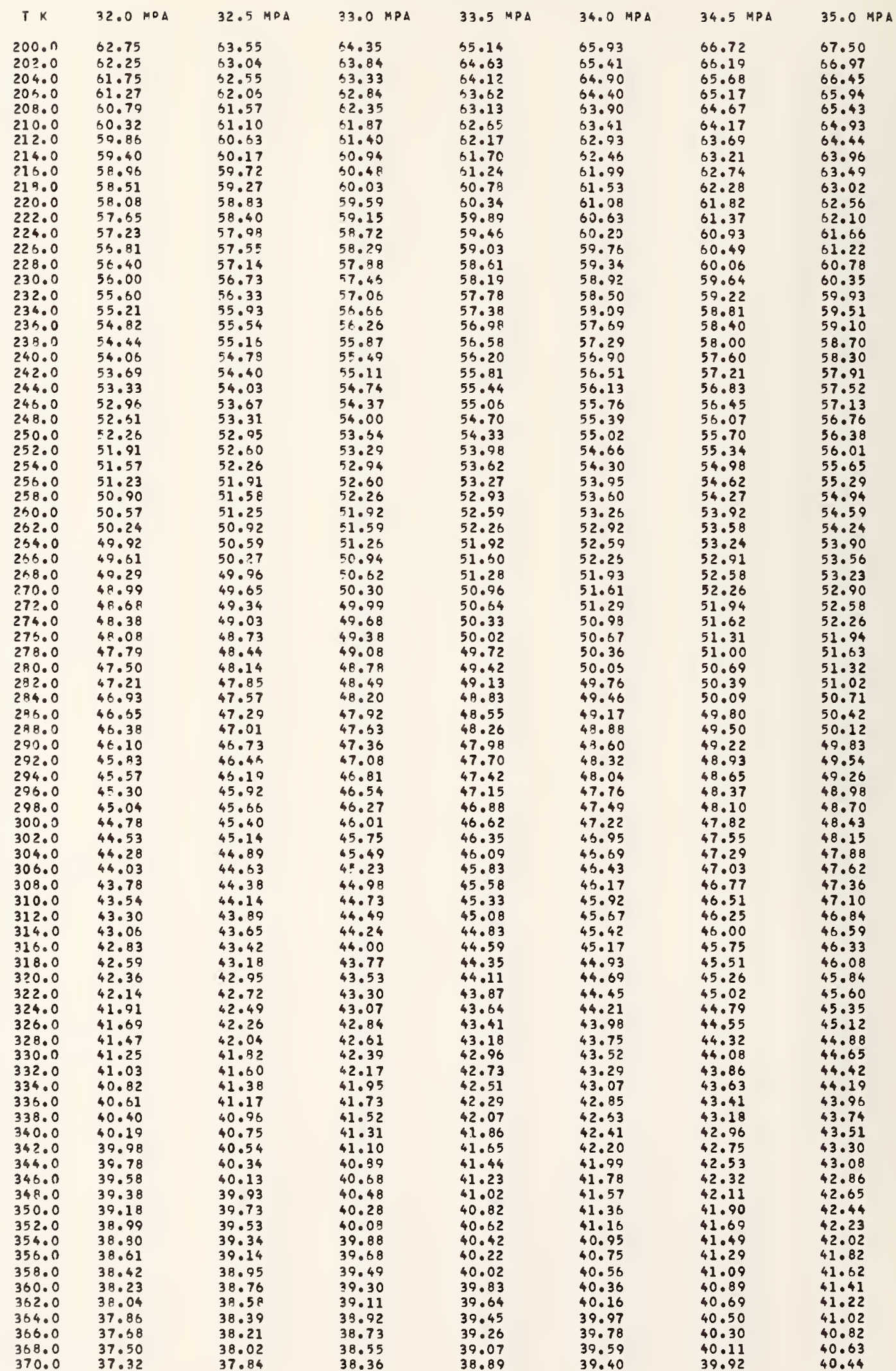


HELIUM DENSITY (KG/M3).

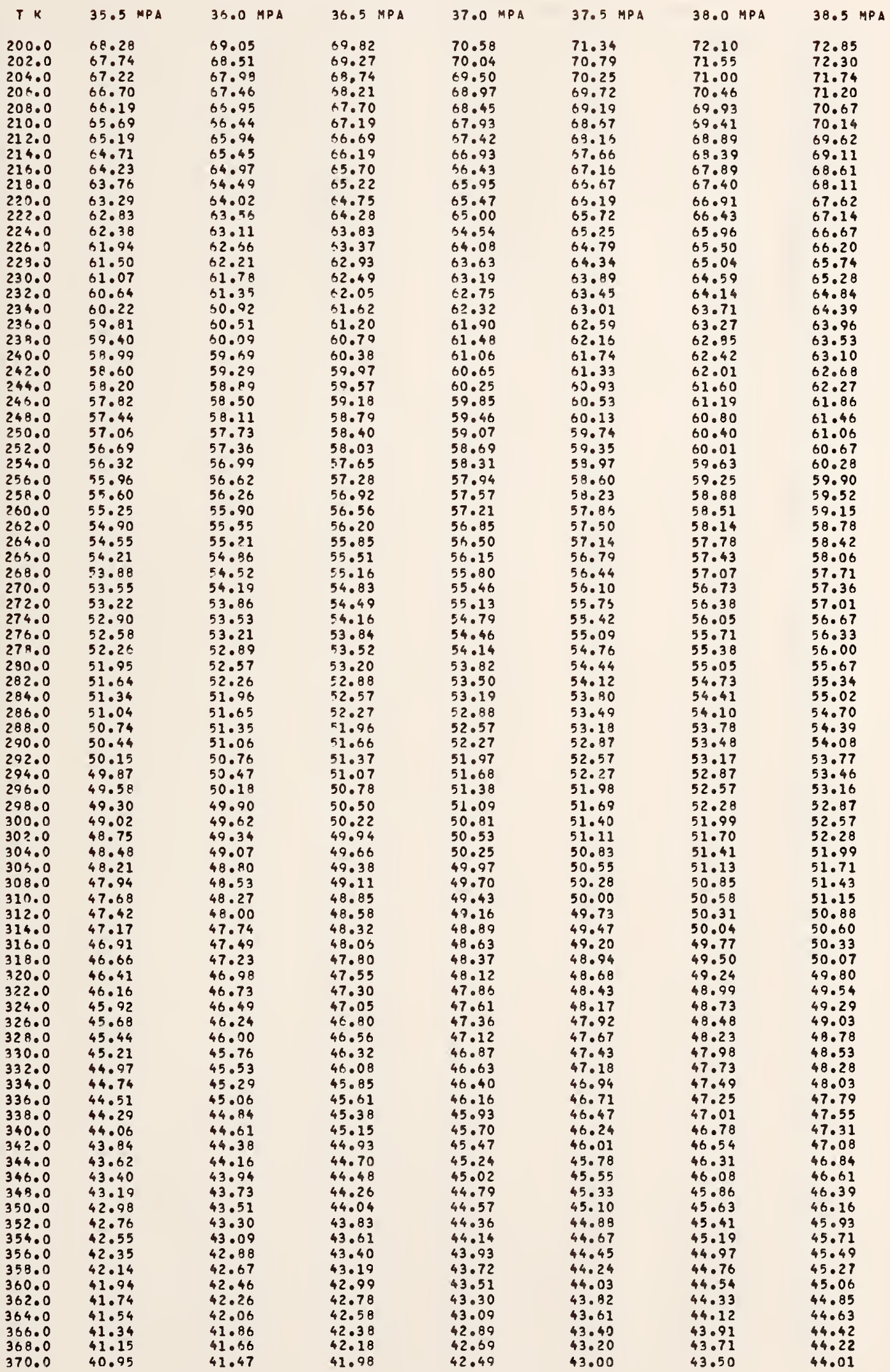


HELIUM DENSITY (KG/43).

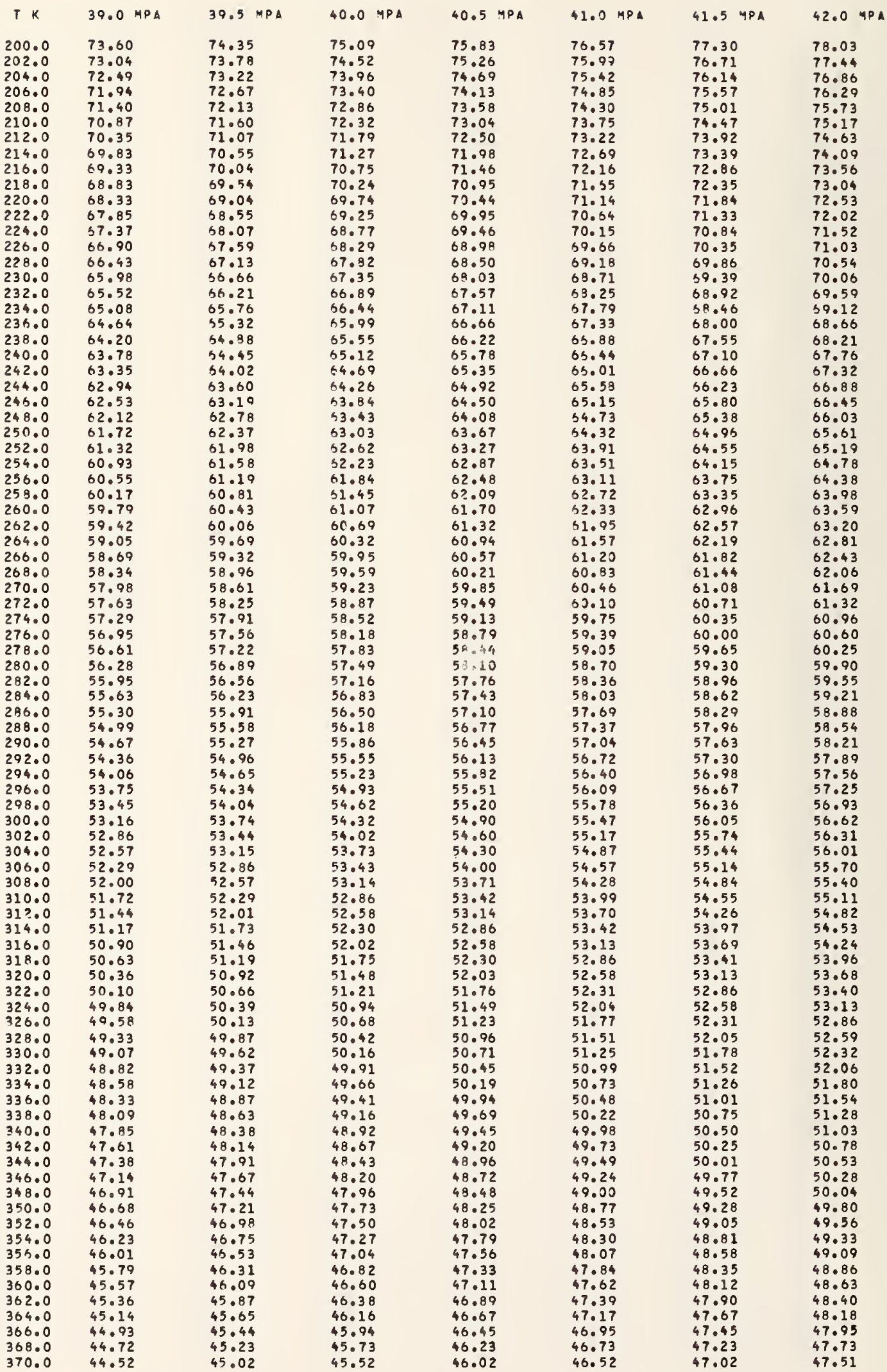


HELIUH OENSITY (KG/MB).

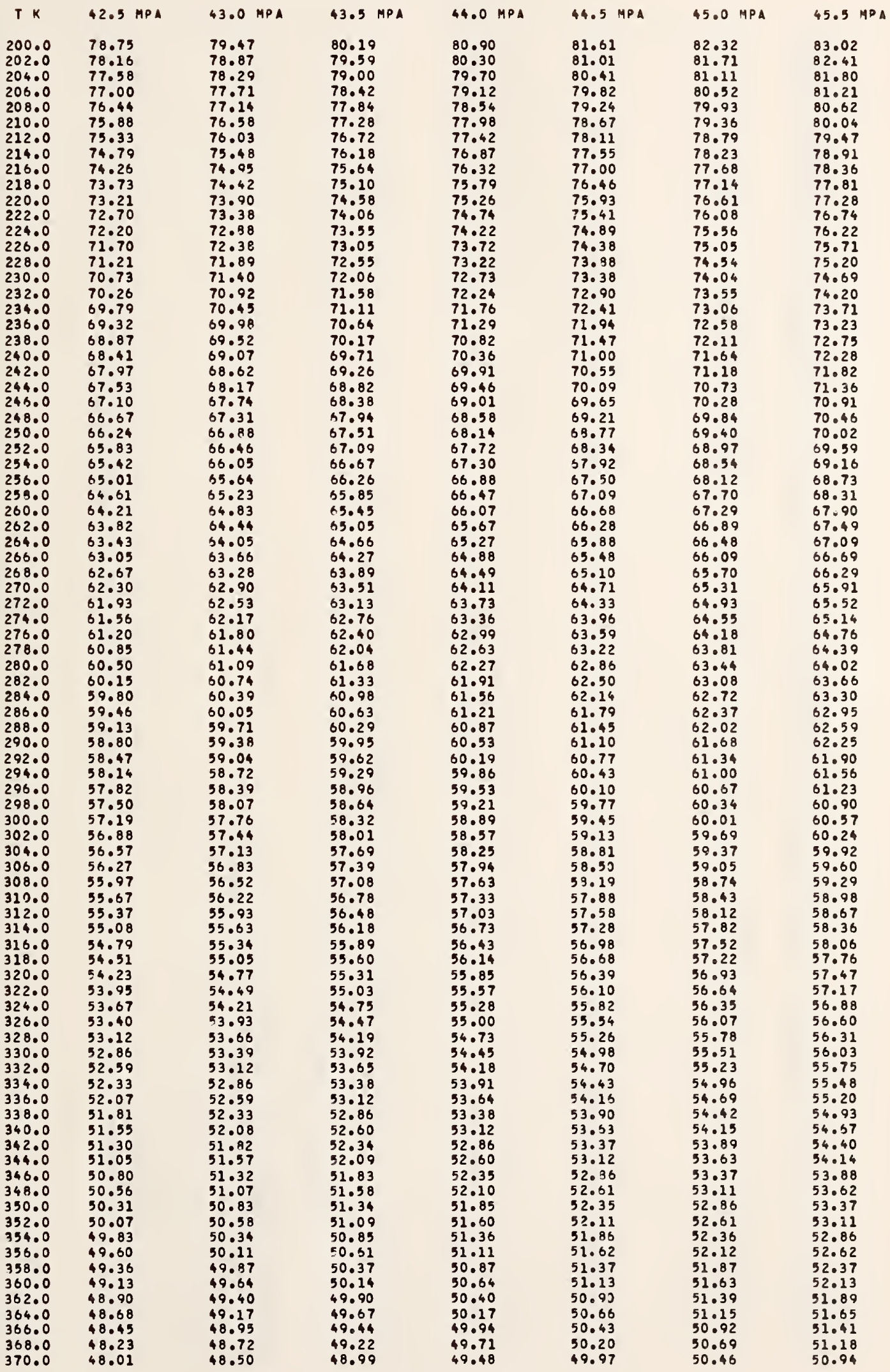


HELIUN DENSITY (KG/M3).

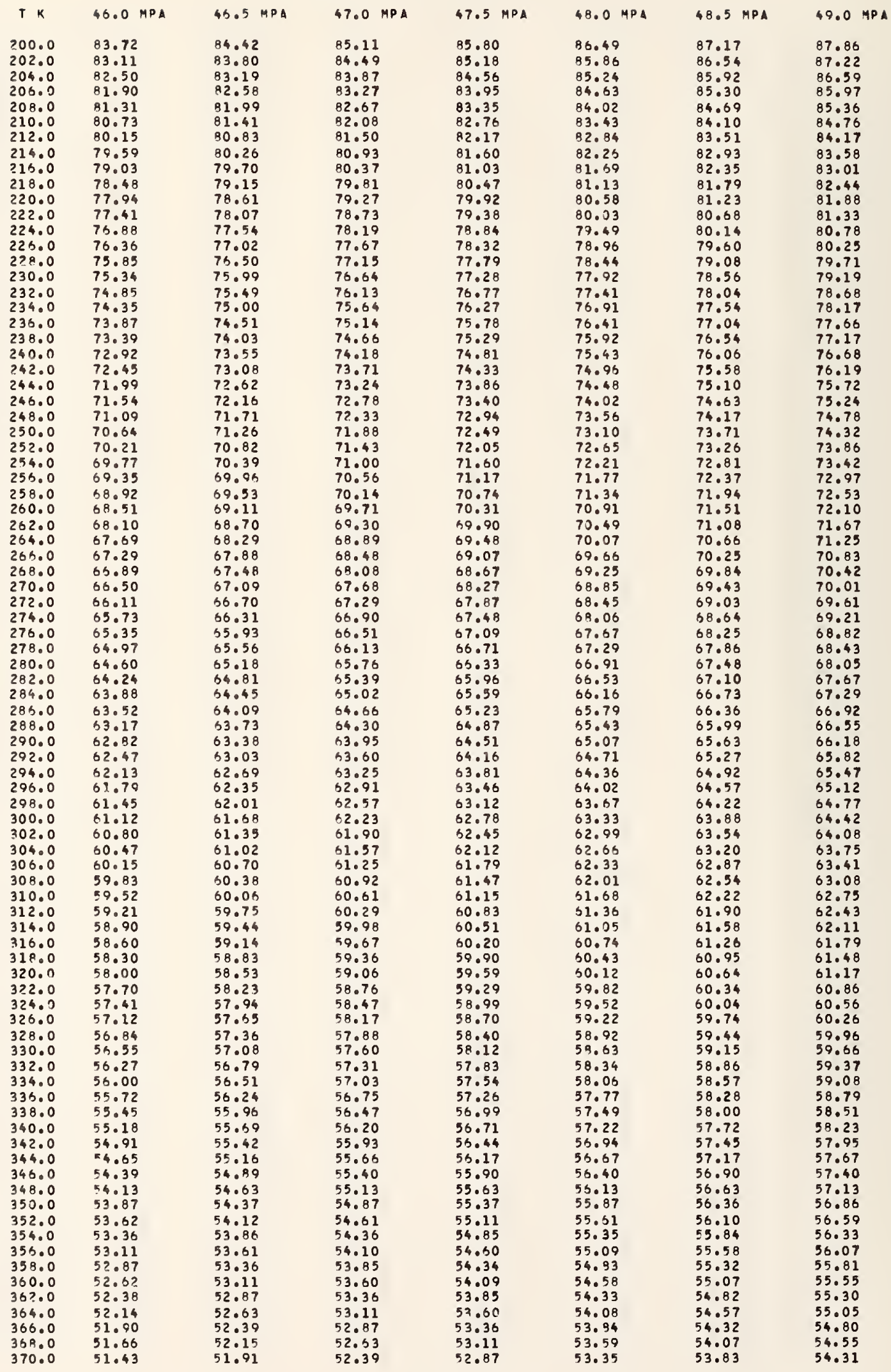


HELIUM DENSITY (KG/M3).

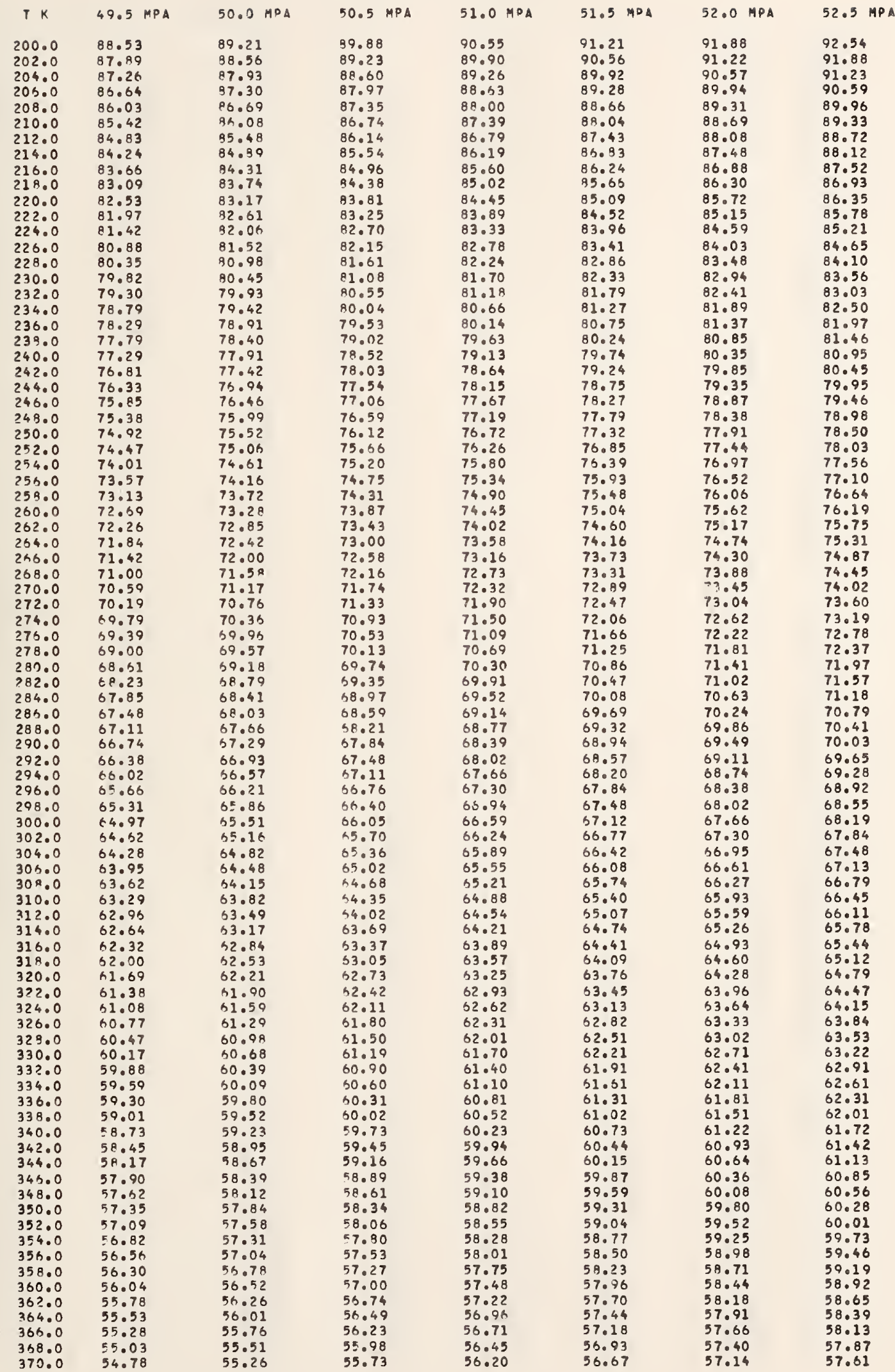


HELIUM DENSITY (KG/MB).

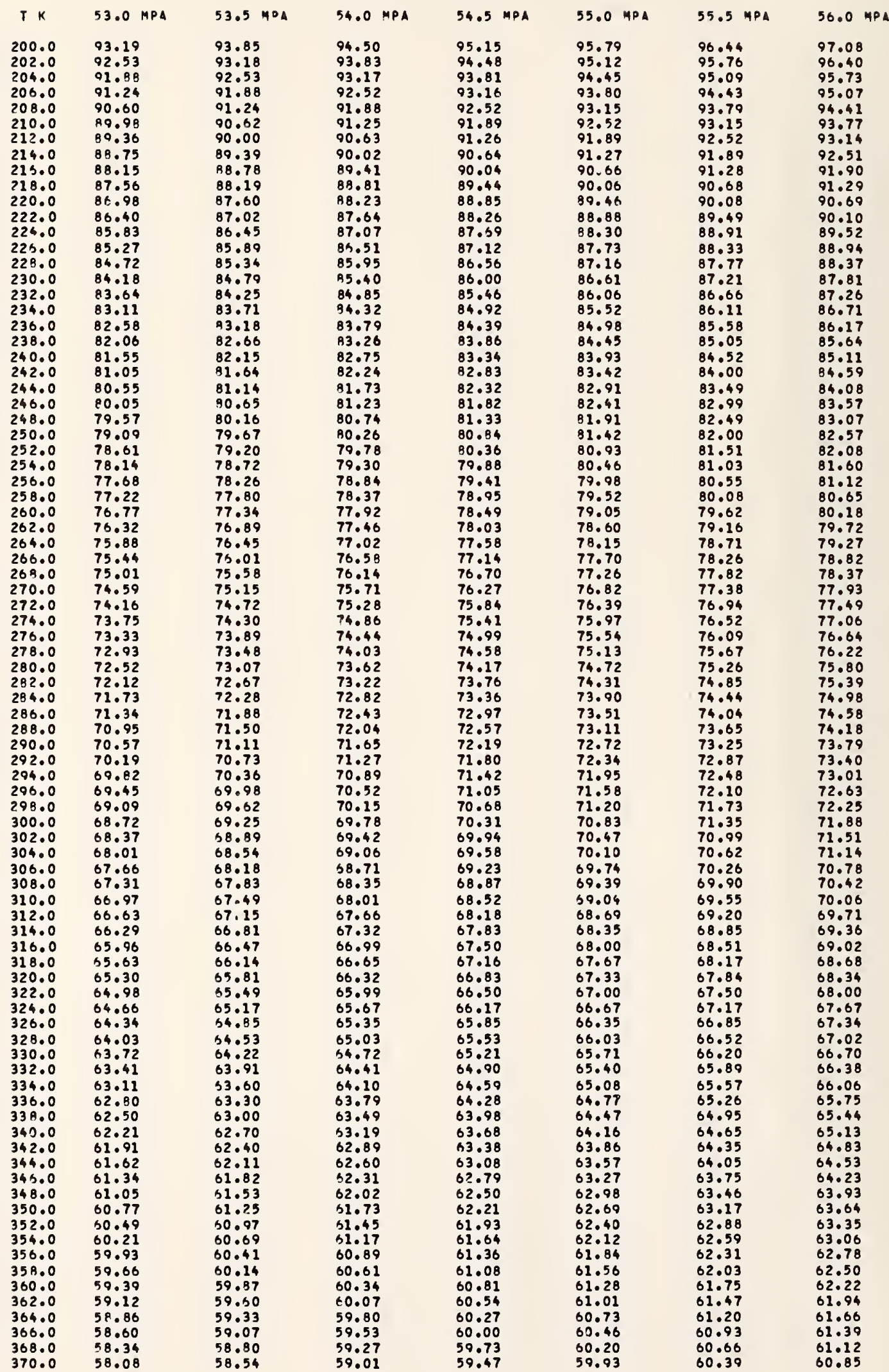


HELIUM DENSITY (KG/M3).

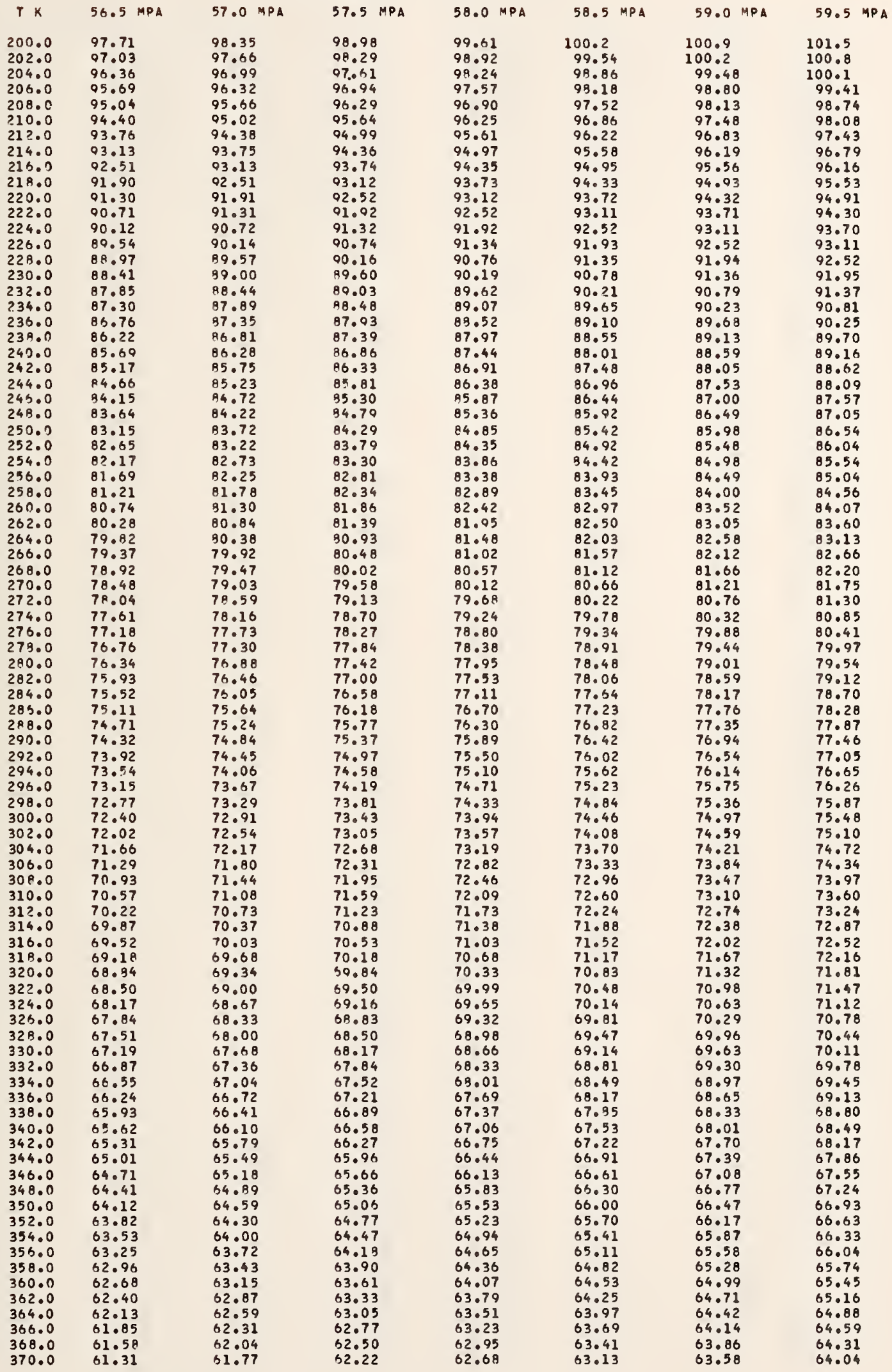


YELIUM OENSITY (KG/M3).

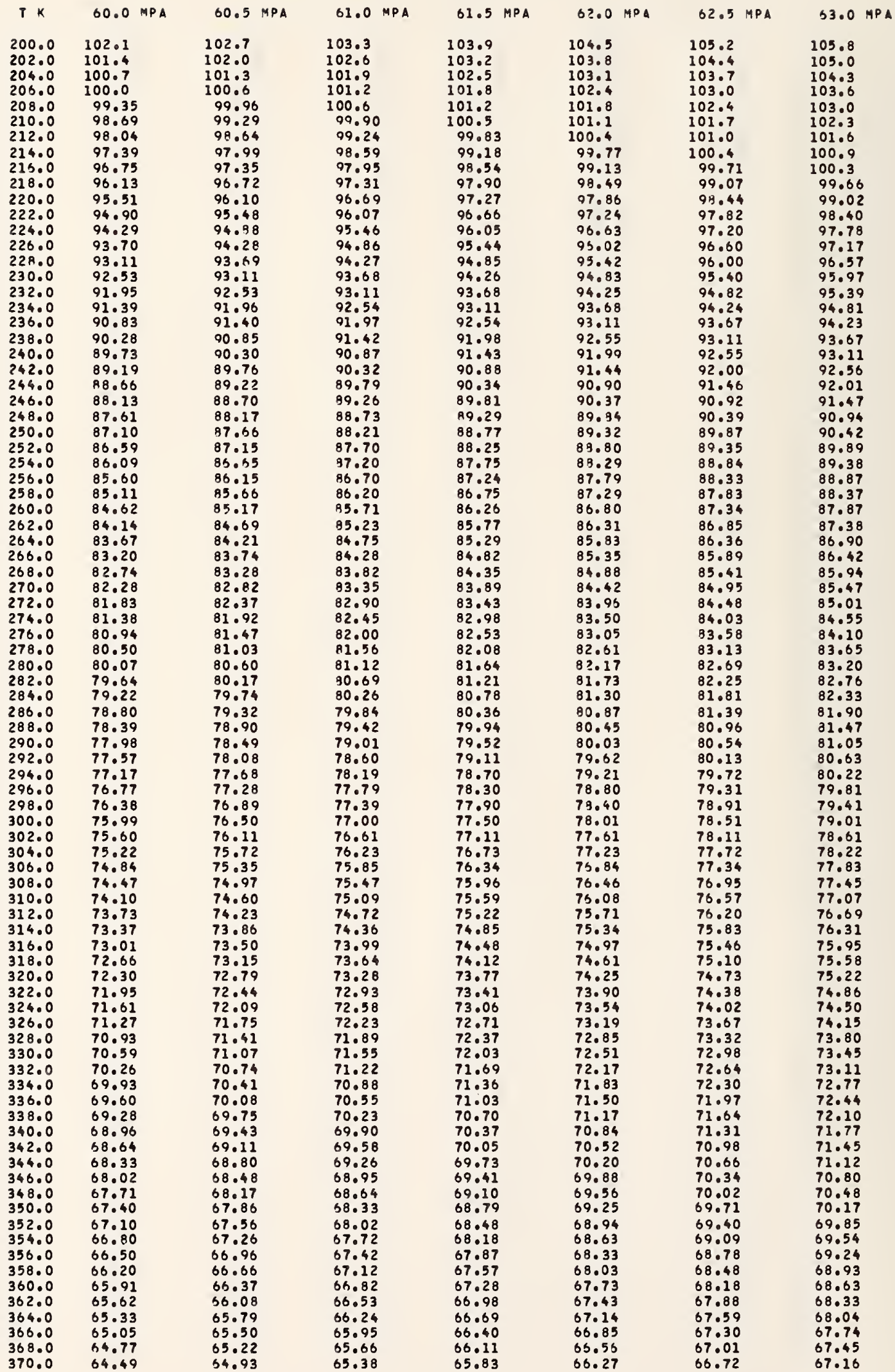


HELIUM OENSITY (KG/M3).

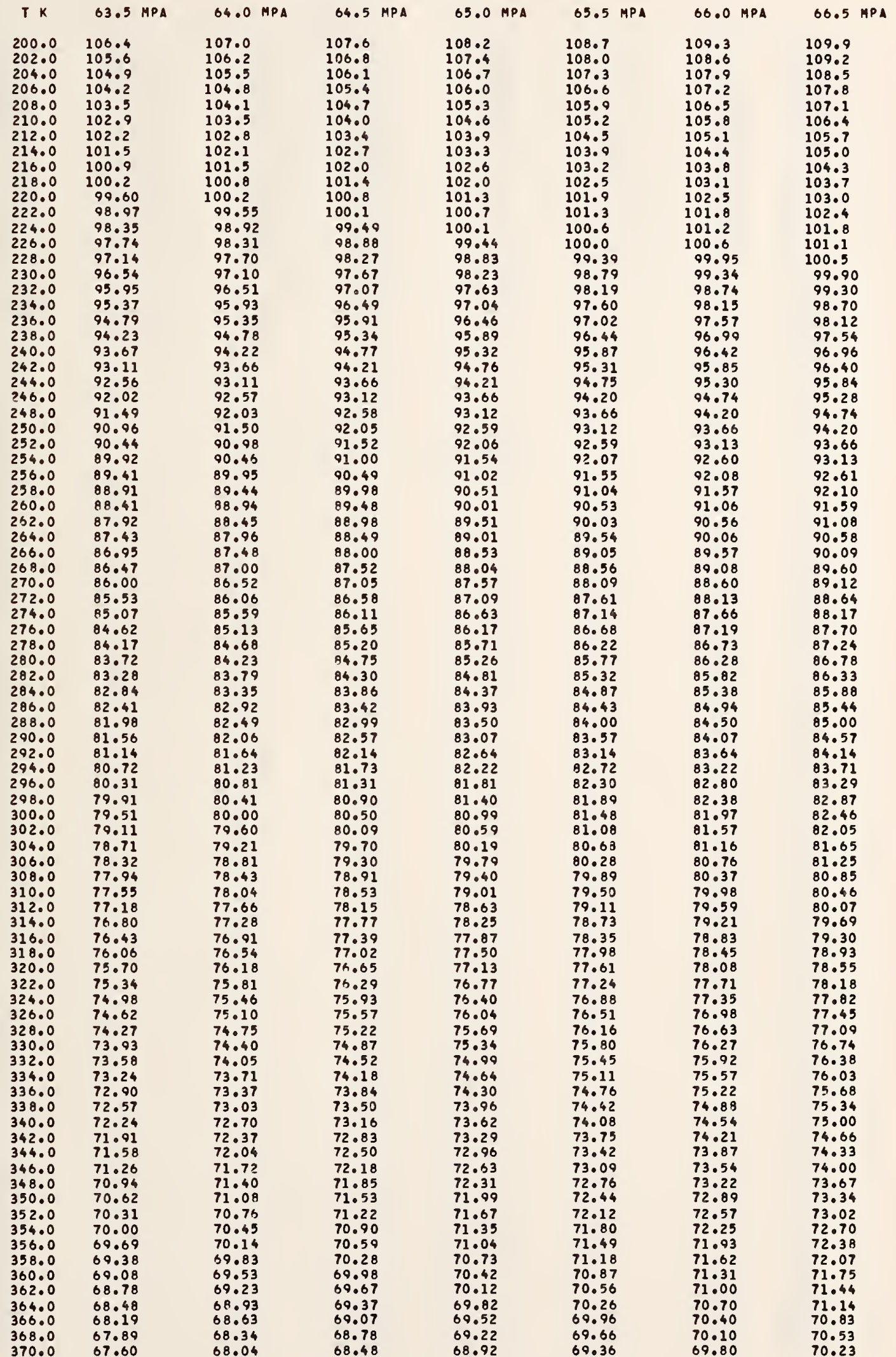


MELIUM OENSITY (KG/M3).

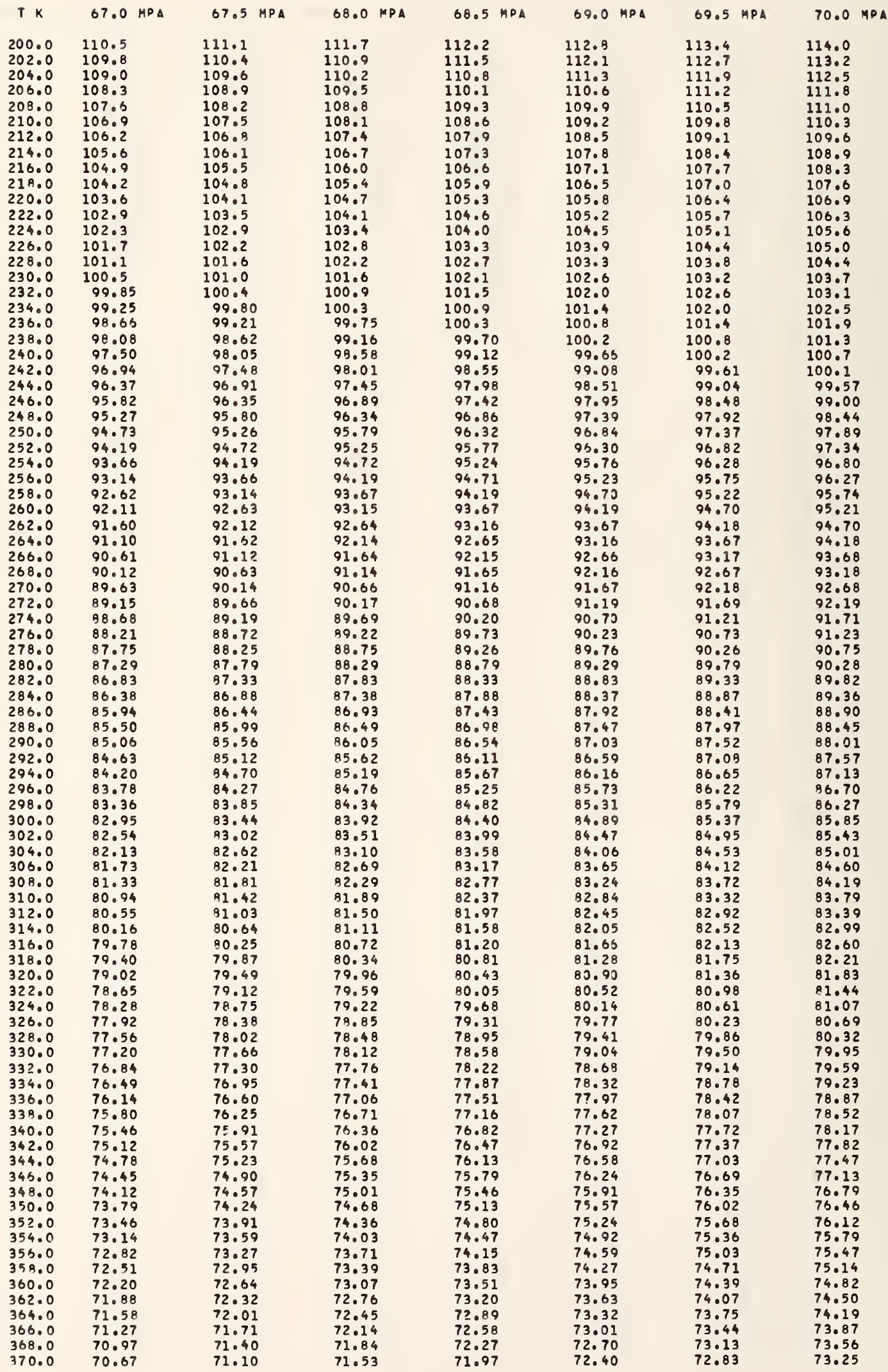




$$
9.5
$$

Hydrogen Density $\left(\mathrm{kg} / \mathrm{m}^{3}\right)$ 
HYDROGEN OENSITY (KG/M3).

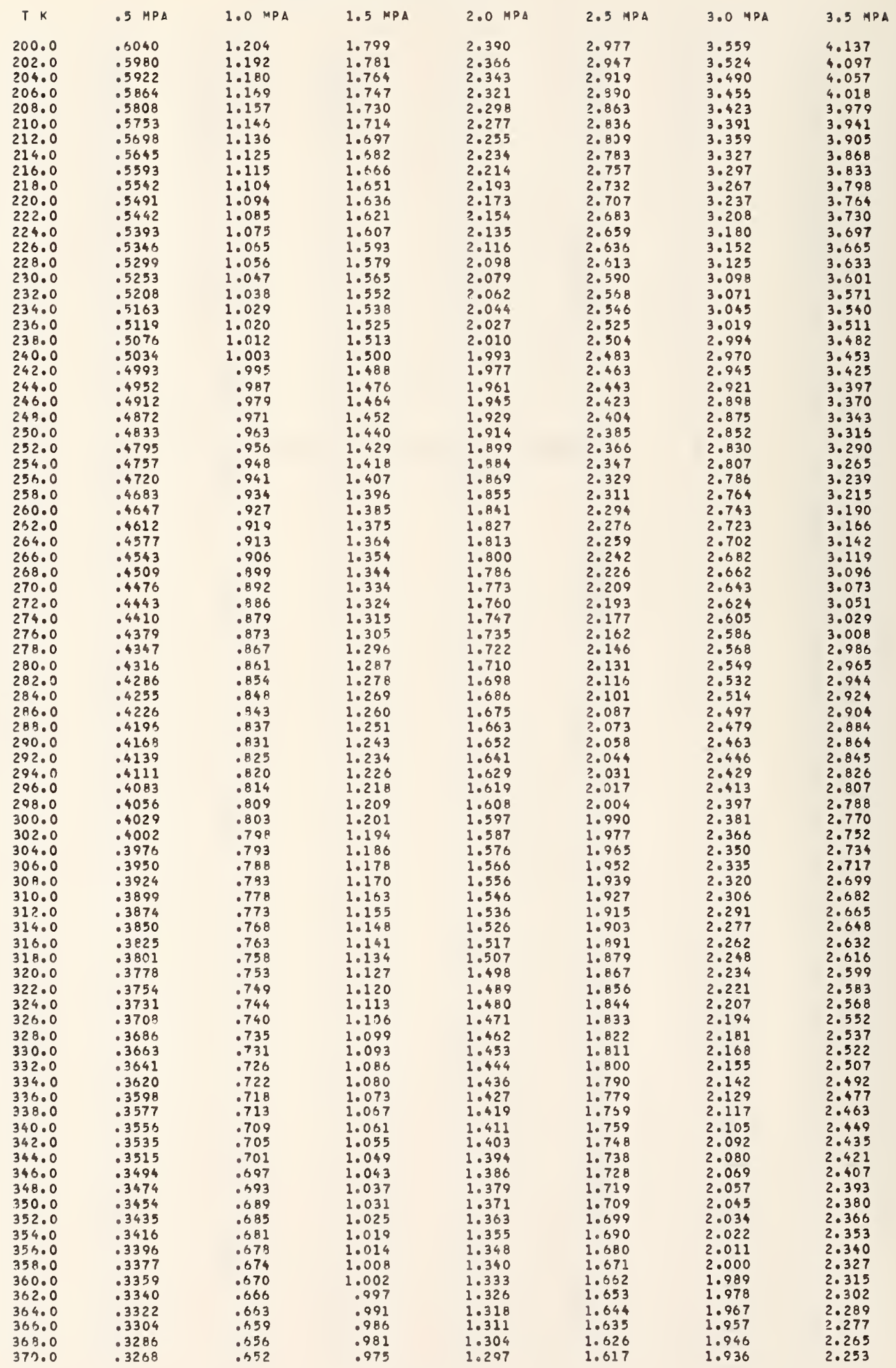


HYDROGEN DENSITY (KG/M3).

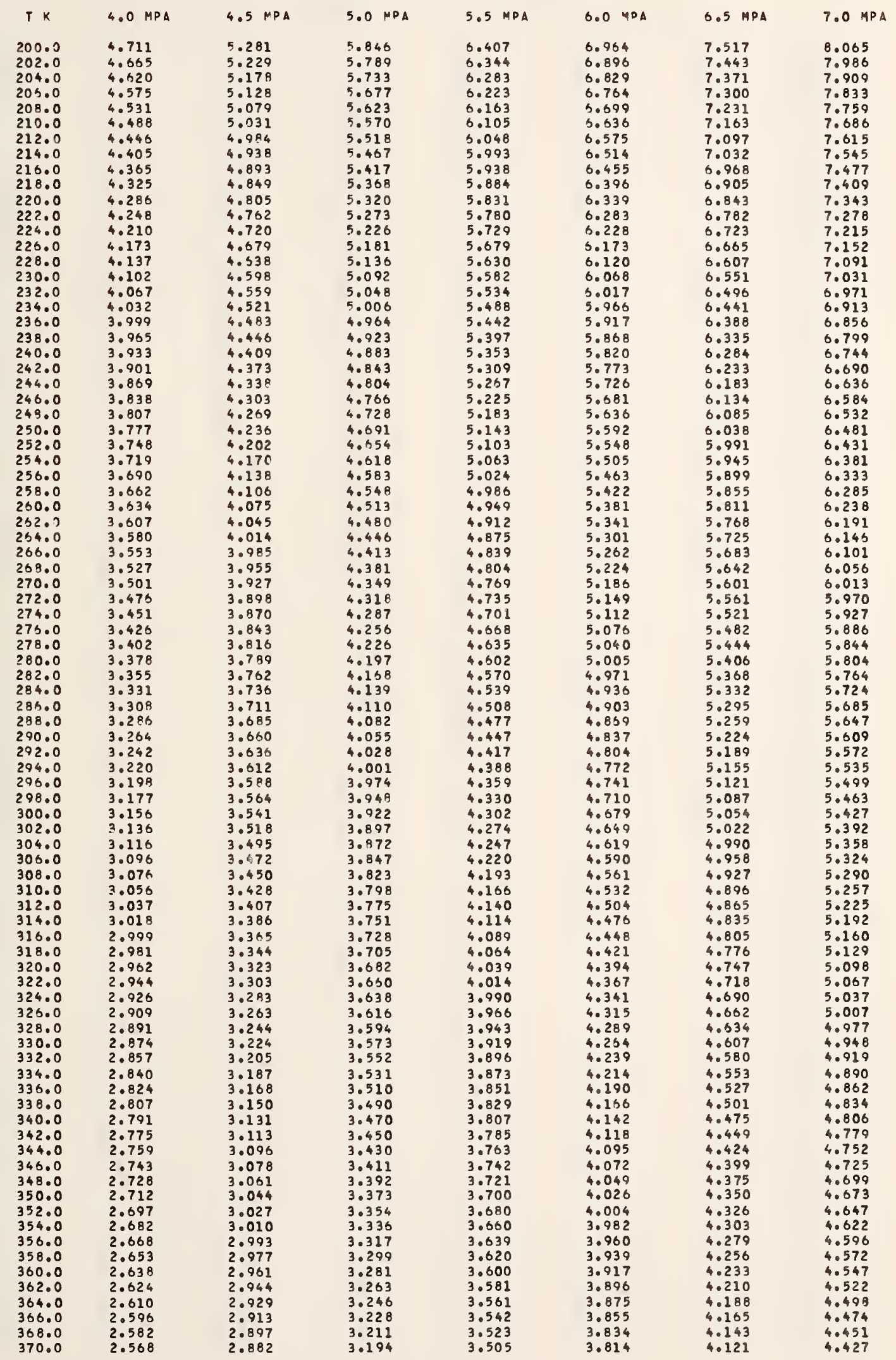


HYOROGEN OENSITY (KGIMB).

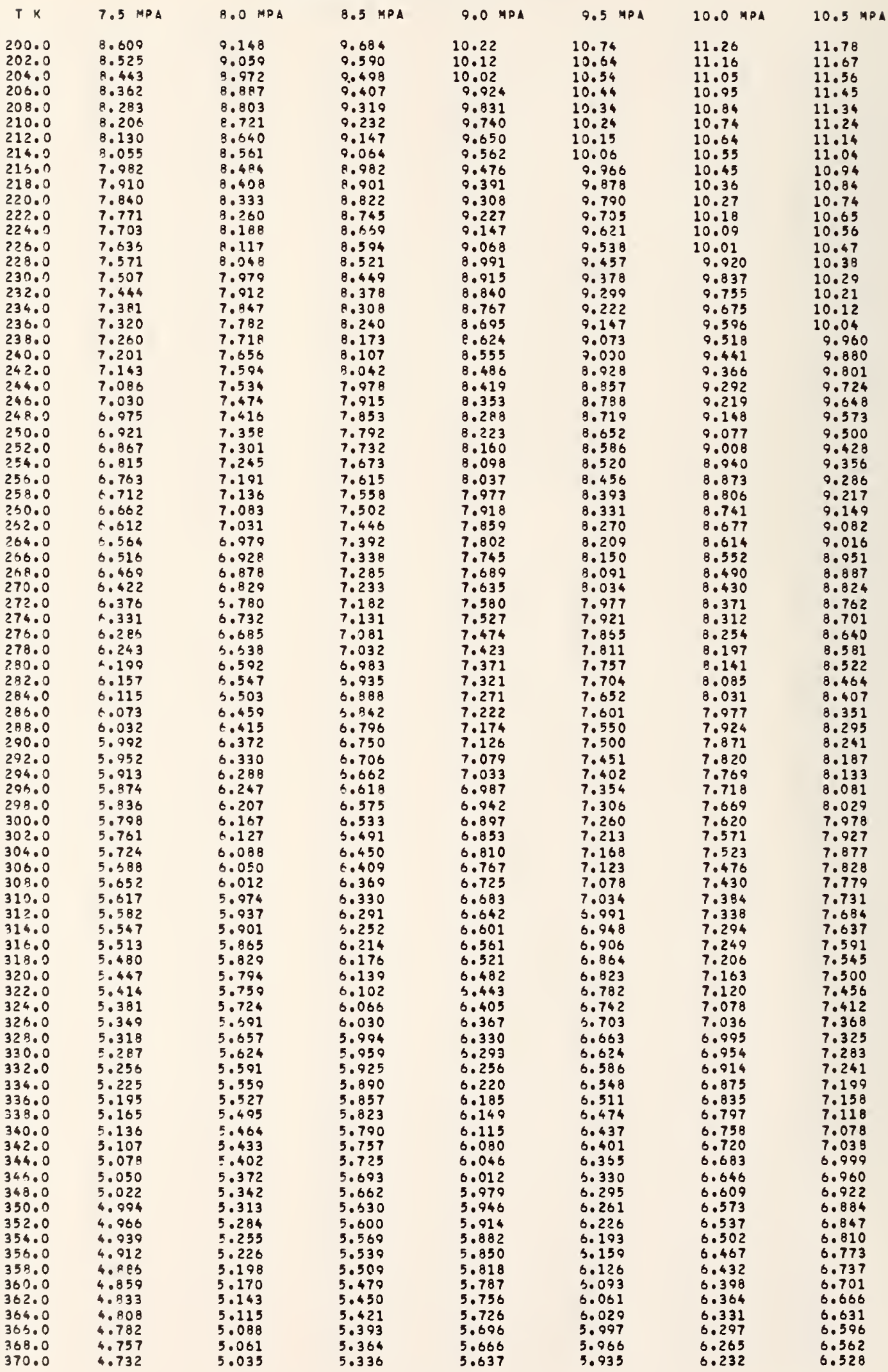


HYORDGEN DENSITY (KG/M3).

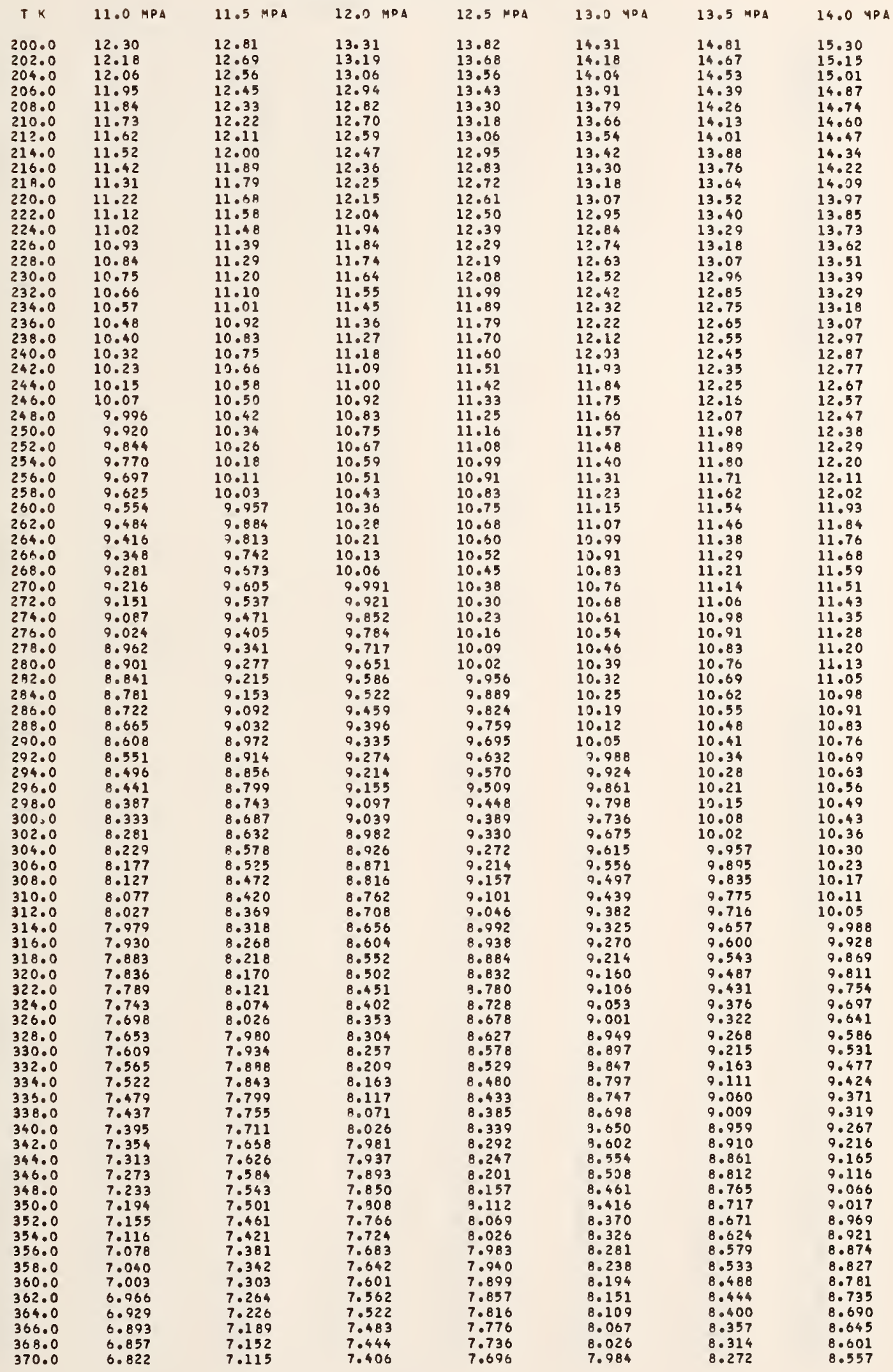


HYOROGEN DENSITY (KG/M3).

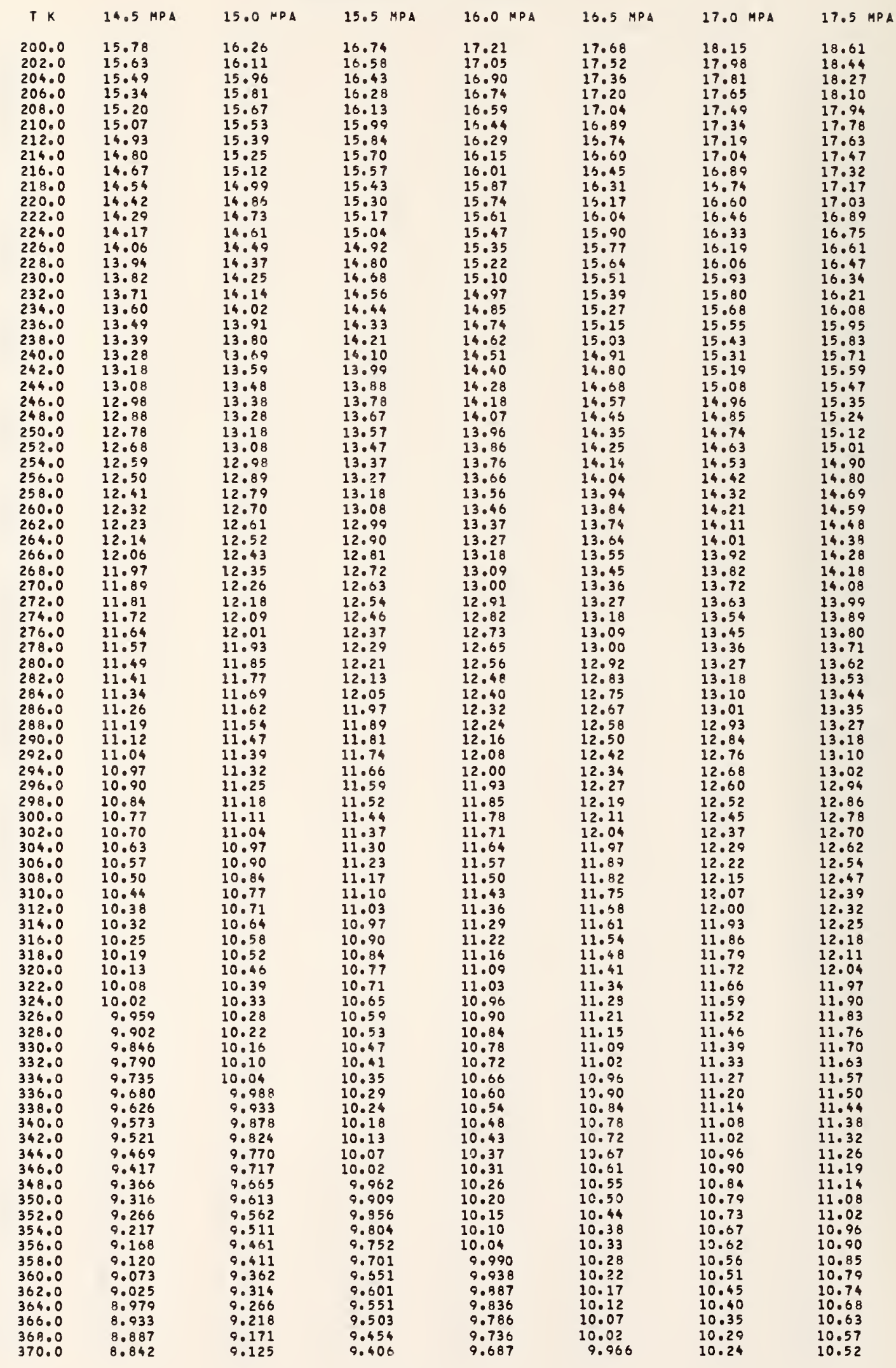


HYDROGEN DENSITY (KG/M3).

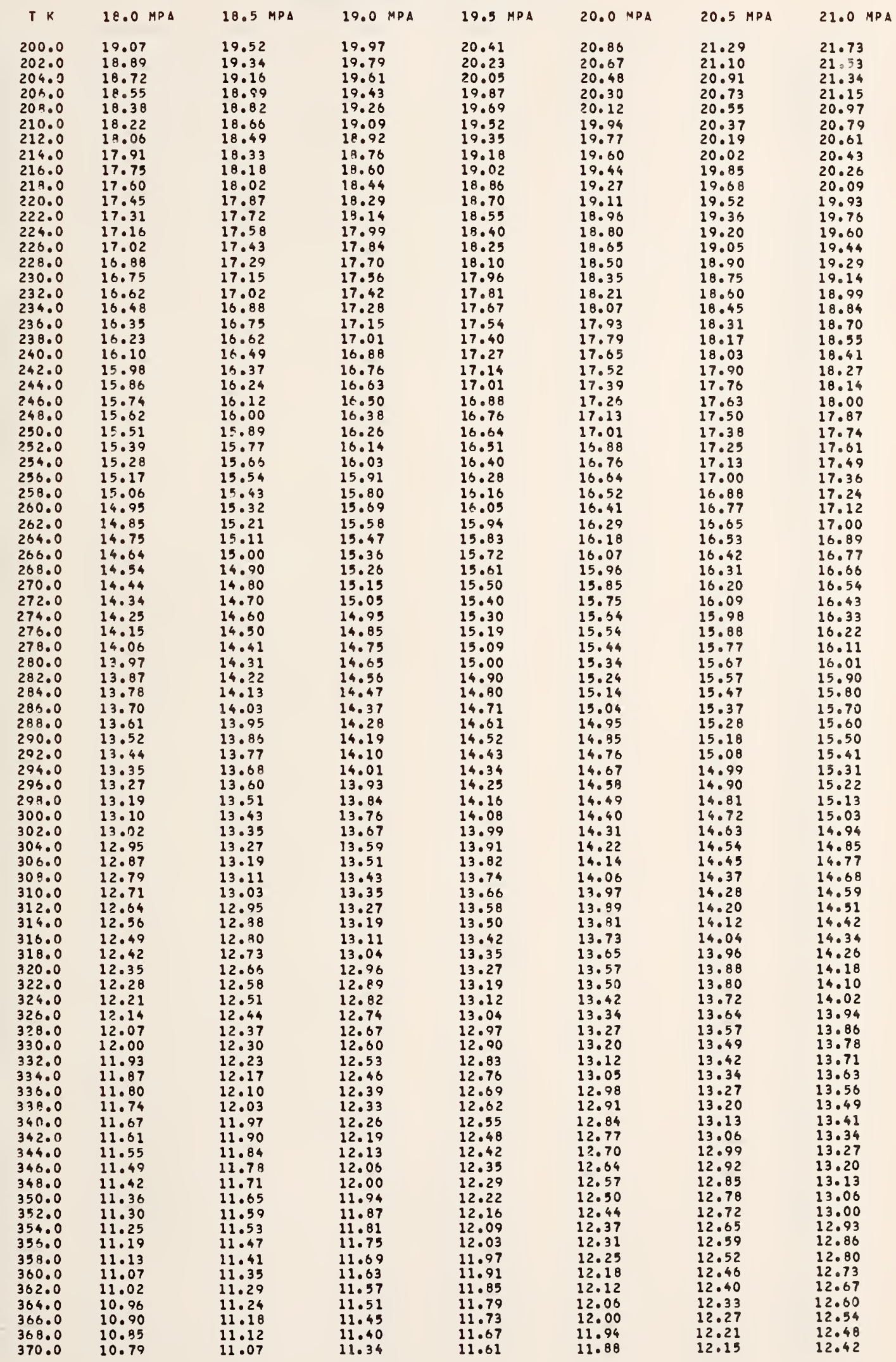


HYORDGEN DENSITY (KG/M3).

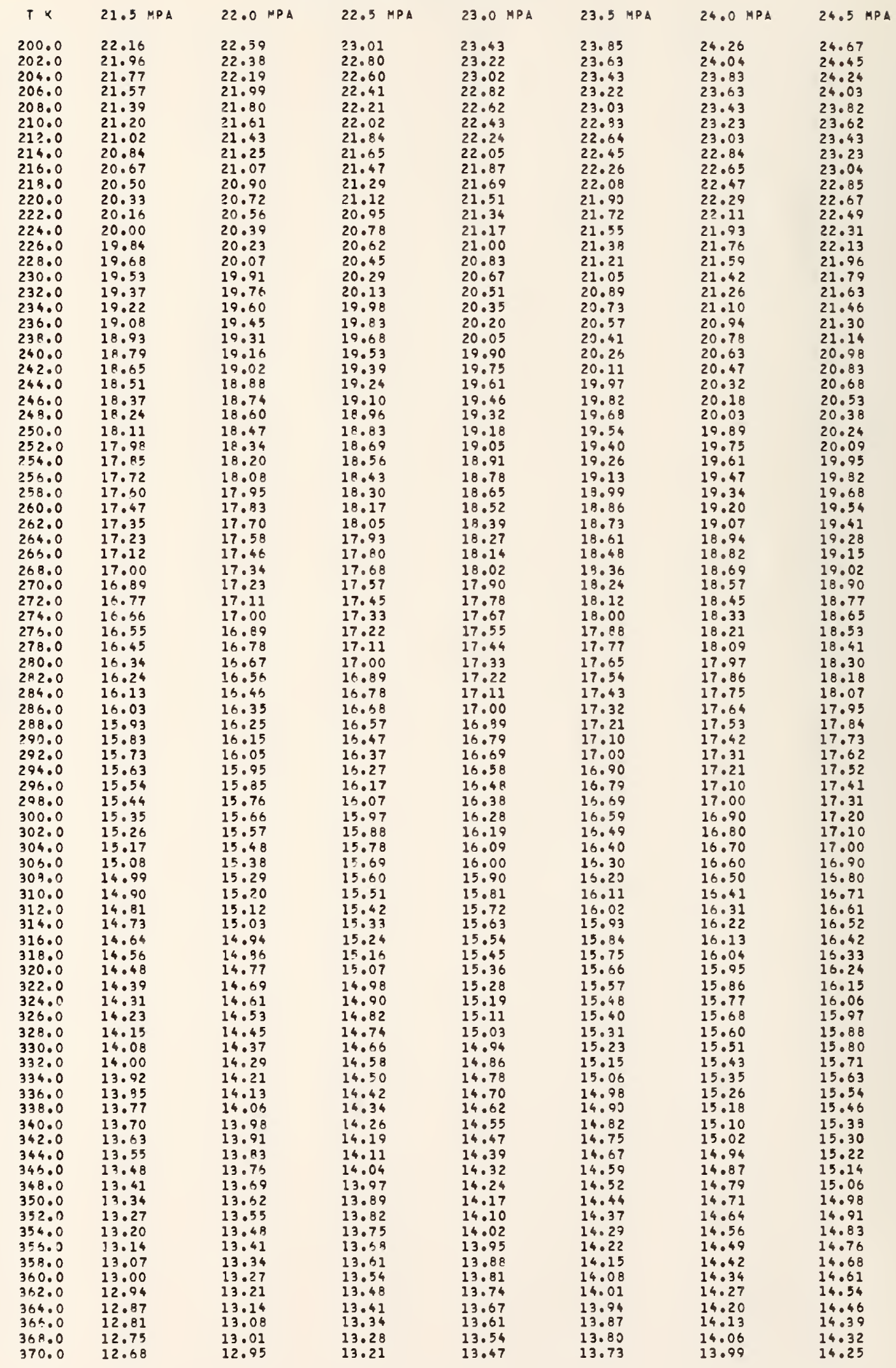


HYDROGEN OENSITY (KG/M3).

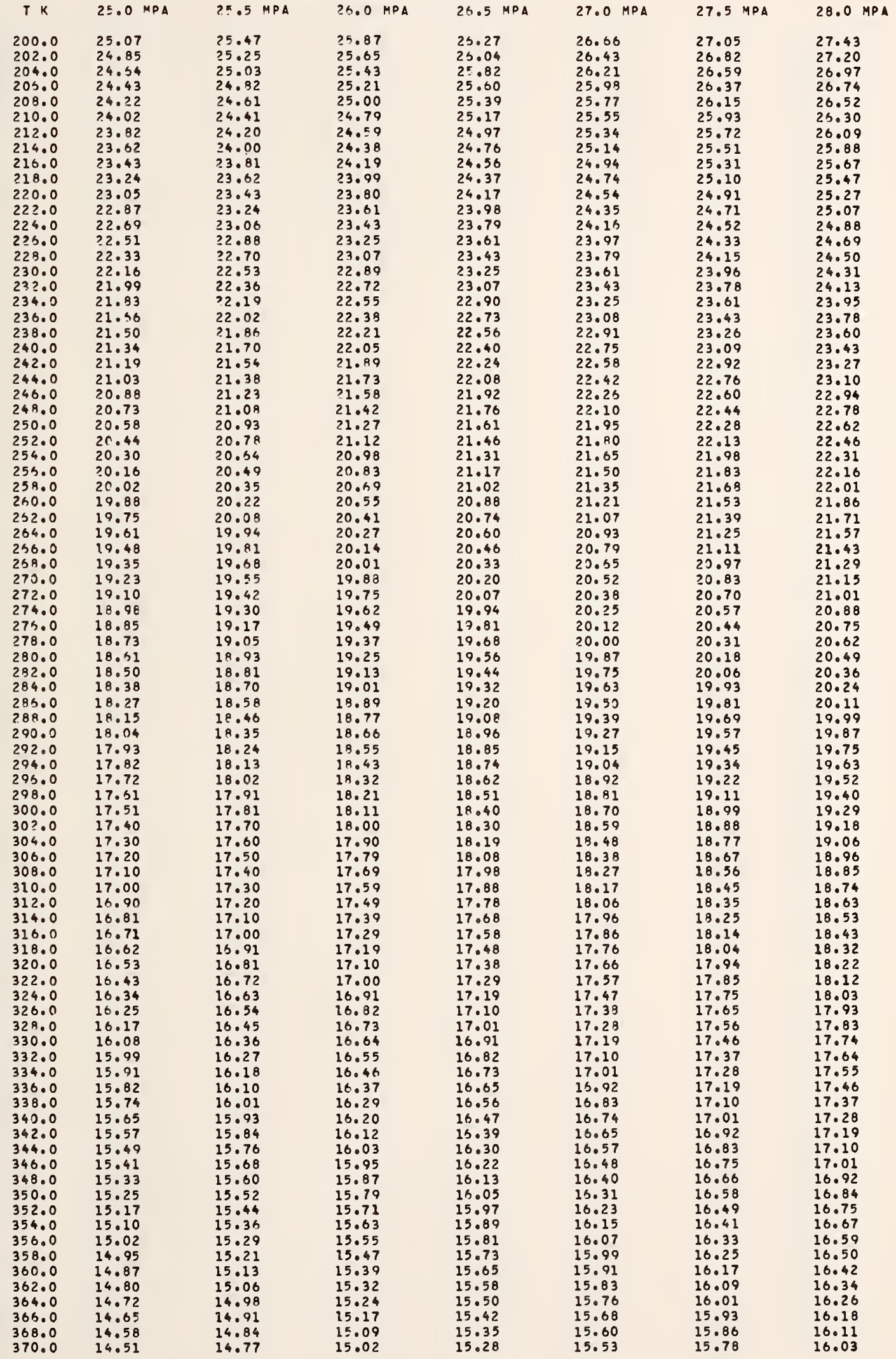


HYOROGEN DENSITY (KG/MB).

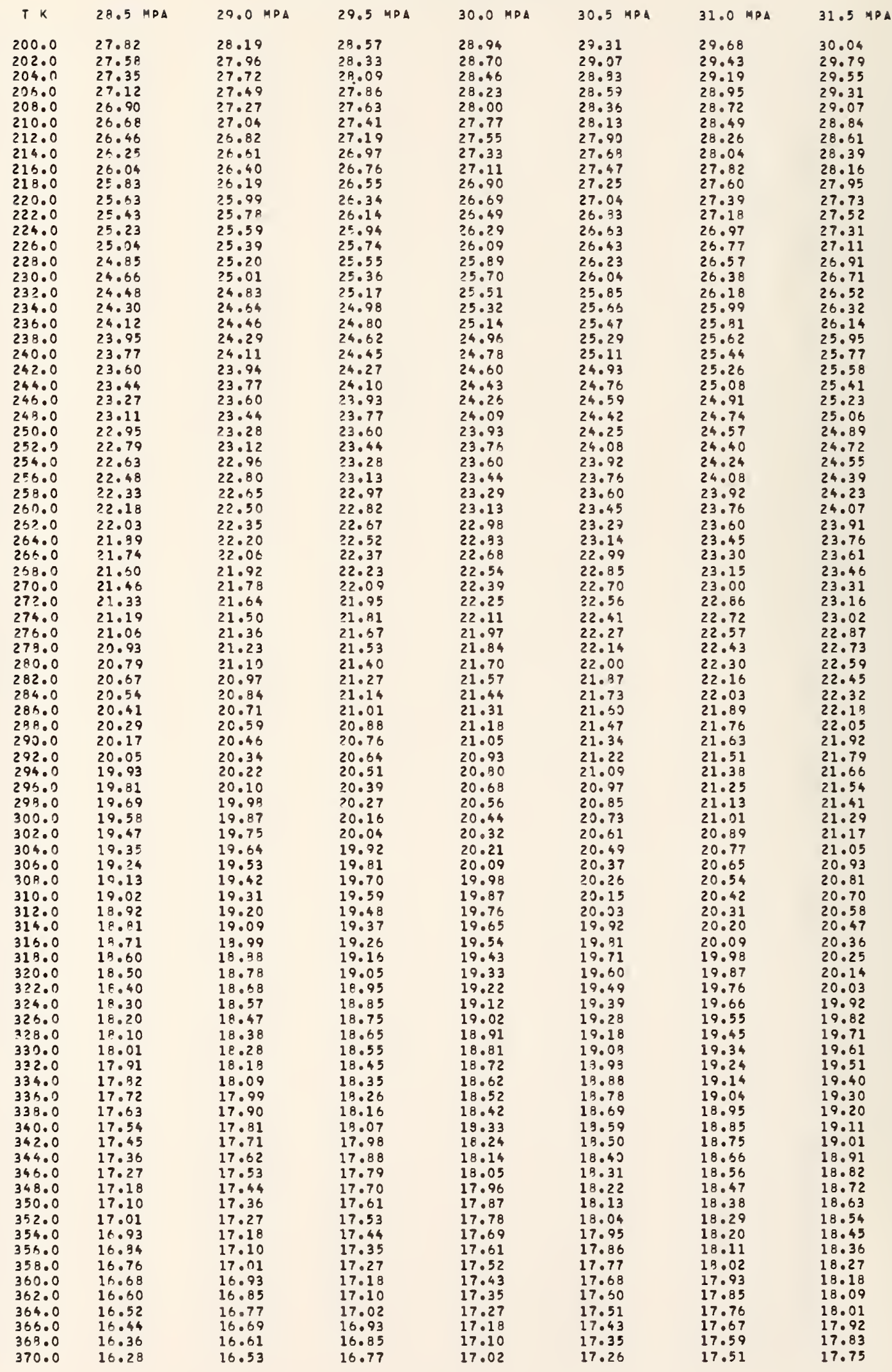


HYORDGEN DENSITY (KG/M3).

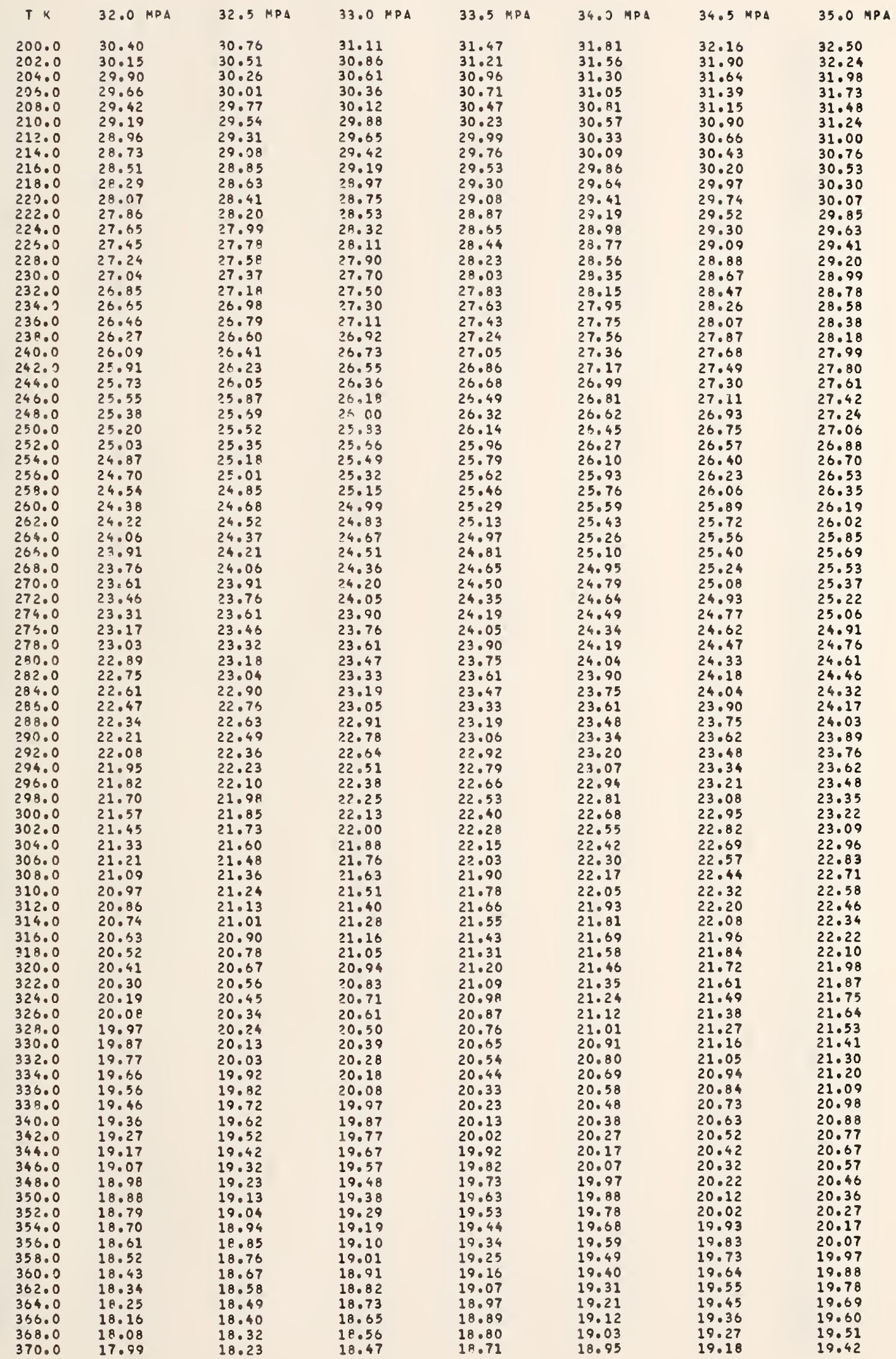


HYOROGEN DENSITY (KG/M3).

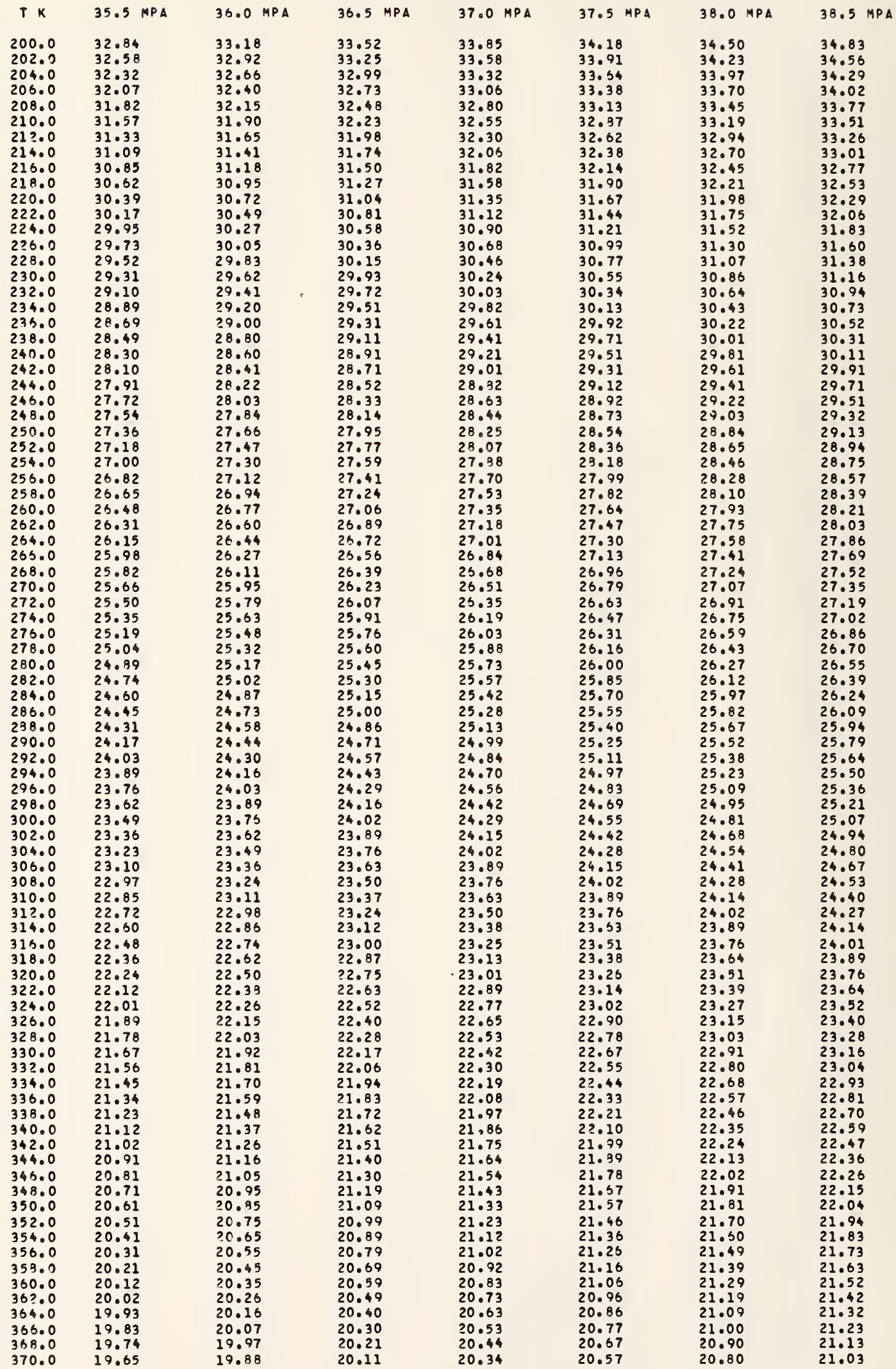


HYOROGEN OENSITY (KG/M3).

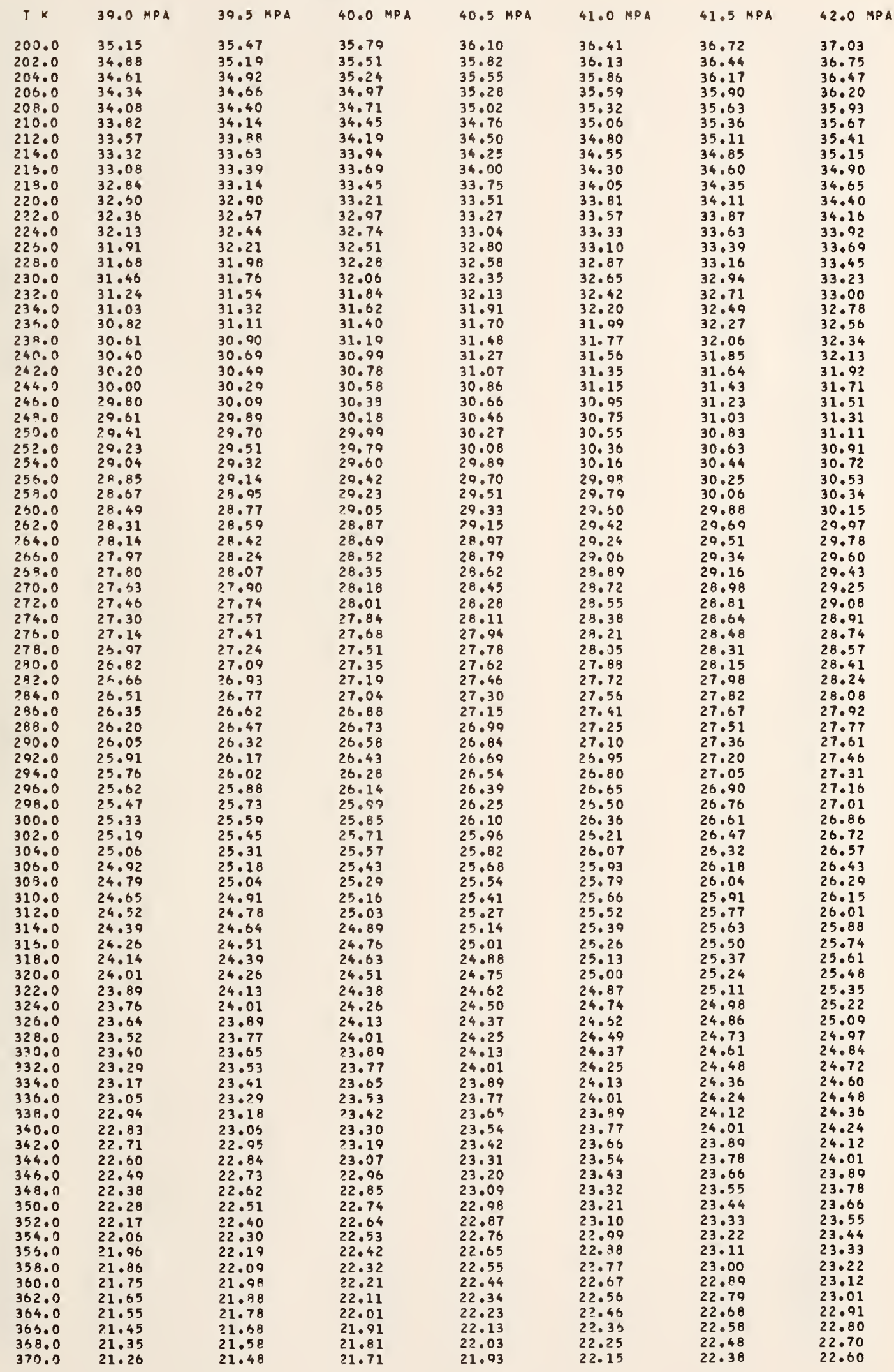


HYDROGEN DENSITY (KG/M3).

\begin{tabular}{|c|c|c|c|c|c|c|c|}
\hline$T K$ & 42.5 MPA & $43.0 \mathrm{MPA}$ & $43.5 \mathrm{MPA}$ & $44.0 \mathrm{MPA}$ & $44.5 \mathrm{MPA}$ & $45.0 \mathrm{MPA}$ & $45.5 \mathrm{MPA}$ \\
\hline $\begin{array}{l}200.0 \\
202.0\end{array}$ & $\begin{array}{l}37.34 \\
37.05\end{array}$ & $\begin{array}{l}37.64 \\
37.035\end{array}$ & $\begin{array}{l}37.94 \\
37.65\end{array}$ & $\begin{array}{l}38.24 \\
37.95\end{array}$ & $\begin{array}{r}38.53 \\
38.25\end{array}$ & $\begin{array}{l}38.83 \\
38.54\end{array}$ & 39.12 \\
\hline $\begin{array}{l}202.0 \\
204.0\end{array}$ & $\begin{array}{l}37.05 \\
35.77\end{array}$ & $\begin{array}{l}37.35 \\
37.07\end{array}$ & $\begin{array}{l}37.65 \\
37.37\end{array}$ & $\begin{array}{l}37.95 \\
37.67\end{array}$ & $\begin{array}{l}38.25 \\
37.96\end{array}$ & $\begin{array}{l}38.54 \\
38.25\end{array}$ & $\begin{array}{l}38.83 \\
38.54\end{array}$ \\
\hline 206.0 & 36.50 & 36.80 & 37.10 & 37.39 & 37.68 & 37.97 & 38.26 \\
\hline 208.0 & 36.23 & 36.53 & 36.82 & 37.12 & 37.41 & 37.70 & 37.99 \\
\hline 210.0 & 35.96 & 36.26 & 36.56 & 36.85 & 37.14 & 37.43 & 37.71 \\
\hline 212.0 & 35.70 & 36.00 & 36.29 & 36.58 & 35.87 & 37.16 & 37.45 \\
\hline 214.0 & 35.45 & 35.74 & 36.03 & 36.32 & 36.61 & 36.90 & 37.18 \\
\hline 213.0 & 35.19 & 35.48 & 35.78 & 36.07 & 36.35 & 36.64 & 36.92 \\
\hline 218.0 & 34.94 & 35.23 & 35.52 & 35.81 & 36.10 & 36.31 & 36.66 \\
\hline $\begin{array}{l}220.0 \\
222.0\end{array}$ & $\begin{array}{l}34.69 \\
34.45\end{array}$ & $\begin{array}{l}34.99 \\
34.74\end{array}$ & $\begin{array}{l}35.27 \\
35.03\end{array}$ & $\begin{array}{l}35.56 \\
35.32\end{array}$ & $\begin{array}{l}35.85 \\
35.60\end{array}$ & $\begin{array}{l}36.13 \\
35.88\end{array}$ & $\begin{array}{l}36.41 \\
36.16\end{array}$ \\
\hline 224.0 & 34.21 & 34.50 & 34.79 & 35.07 & 35.36 & 35.64 & 33.92 \\
\hline 226.0 & 33.98 & 34.26 & 34.55 & 34.83 & 35.11 & 35.39 & 35.67 \\
\hline 228.0 & 33.74 & 34.03 & 34.31 & 34.60 & 34.88 & 35.16 & 35.43 \\
\hline 230.0 & 33.51 & 33.80 & 34.08 & 34.36 & 34.64 & 34.92 & 35.20 \\
\hline 232.0 & 33.29 & 33.57 & 33.85 & 34.13 & 34.41 & 34.69 & 34.96 \\
\hline 234.0 & 33.06 & 33.35 & 33.63 & 33.91 & 34.18 & 34.46 & 34.73 \\
\hline $\begin{array}{l}236.0 \\
238.0\end{array}$ & $\begin{array}{l}32.84 \\
32.53\end{array}$ & $\begin{array}{l}33.13 \\
32.91\end{array}$ & $\begin{array}{l}33.41 \\
33.19\end{array}$ & $\begin{array}{l}33.68 \\
33.46\end{array}$ & $\begin{array}{l}33.96 \\
33.74\end{array}$ & $\begin{array}{l}34.24 \\
34.01\end{array}$ & $\begin{array}{l}34.51 \\
34.28\end{array}$ \\
\hline 240.0 & 32.41 & 32.69 & 32.97 & 33.25 & 33.52 & 33.79 & 34.06 \\
\hline 242.0 & 32.20 & 32.48 & 32.76 & 33.03 & 33.30 & 33.58 & 33.85 \\
\hline $\begin{array}{l}244.0 \\
245.0\end{array}$ & $\begin{array}{r}31.99 \\
31.79\end{array}$ & 32.27 & 32.55 & $32 \cdot 82$ & 33.09 & 33.36 & 33.63 \\
\hline $\begin{array}{l}243.0 \\
243.0\end{array}$ & $\begin{array}{l}31.79 \\
31.58\end{array}$ & $\begin{array}{l}32.06 \\
31.96\end{array}$ & $\begin{array}{l}32.34 \\
32.13\end{array}$ & $\begin{array}{l}32.61 \\
32.40\end{array}$ & $\begin{array}{l}32.88 \\
32.67\end{array}$ & $\begin{array}{l}33.15 \\
32.94\end{array}$ & $\begin{array}{l}33.42 \\
33.21\end{array}$ \\
\hline 250.0 & 31.38 & 31.66 & 31.93 & 32.20 & 32.47 & 32.74 & 33.00 \\
\hline $25 ? .0$ & 31.19 & 31.46 & 31.73 & 32.00 & 32.27 & 32.53 & 32.80 \\
\hline 254.0 & 30.99 & 31.26 & 31.53 & 31.80 & 32.07 & 32.33 & 32.50 \\
\hline 256.0 & 30.80 & 31.07 & 31.34 & 31.61 & 31.87 & 32.14 & 32.40 \\
\hline 258.0 & 30.61 & 30.88 & 31.15 & 31.41 & 31.68 & 31.94 & 32.20 \\
\hline 260.0 & 30.42 & 30.59 & 30.96 & 31.22 & 31.49 & 31.75 & 32.01 \\
\hline 262.0 & 30.23 & 30.50 & 30.77 & 31.03 & 31.30 & 31.56 & 31.82 \\
\hline 264.0 & 30.05 & 30.32 & 30.58 & 30.85 & 31.11 & 31.37 & 31.63 \\
\hline 265.0 & 29.87 & 30.14 & 30.40 & 30.66 & 30.92 & 31.18 & 31.44 \\
\hline 268.0 & 29.69 & 29.96 & 30.22 & 30.48 & 30.74 & 31.00 & 31.26 \\
\hline 270.0 & 29.52 & 29.78 & 30.04 & 30.30 & 30.56 & 30.82 & 31.08 \\
\hline 272.0 & 29.34 & 29.60 & 29.87 & 30.13 & 30.38 & 30.64 & 30.90 \\
\hline 274.0 & 29.17 & 29.43 & 29.59 & 29.95 & 30.21 & 30.46 & 30.72 \\
\hline 276.0 & 29.00 & 29.26 & 29.52 & 29.78 & 30.03 & 30.29 & 30.54 \\
\hline $\begin{array}{l}273.0 \\
280.0\end{array}$ & 28.83 & 29.09 & $? 9.35$ & 29.61 & 29.86 & 30.12 & 30.37 \\
\hline 280.0 & 28.67 & 28.93 & 29.18 & 29.44 & 29.69 & 29.94 & 30.20 \\
\hline $\begin{array}{l}282.0 \\
284.0\end{array}$ & 28.50 & 28.76 & 29.02 & 29.27 & 29.52 & 29.78 & 30.03 \\
\hline $\begin{array}{l}284.0 \\
286.0\end{array}$ & $\begin{array}{l}28.34 \\
28.18\end{array}$ & $\begin{array}{l}38.60 \\
28.44\end{array}$ & $\begin{array}{l}28.85 \\
28.69\end{array}$ & $\begin{array}{l}29.11 \\
28.94\end{array}$ & $\begin{array}{l}29.36 \\
29.19\end{array}$ & $\begin{array}{l}29.61 \\
29.44\end{array}$ & $\begin{array}{l}29.86 \\
29.69\end{array}$ \\
\hline $28 A_{0} 0$ & 28.02 & 28.28 & 28.53 & 28.78 & 29.03 & 29.28 & 29.53 \\
\hline $\begin{array}{l}290.0 \\
29 ? .0\end{array}$ & $\begin{array}{l}27.87 \\
27.71\end{array}$ & $\begin{array}{l}28.12 \\
27.96\end{array}$ & 28.37 & $\begin{array}{l}28.62 \\
28.47\end{array}$ & 28.87 & 29.12 & 29.37 \\
\hline $\begin{array}{l}292.0 \\
294.0\end{array}$ & & $\begin{array}{l}27.96 \\
27.81\end{array}$ & $\begin{array}{l}28.22 \\
28.06\end{array}$ & & $\begin{array}{l}28.71 \\
28.55\end{array}$ & $\begin{array}{l}28.96 \\
28.80\end{array}$ & $\begin{array}{l}29.21 \\
29.05\end{array}$ \\
\hline 295.0 & 27.41 & 27.66 & $? 7.91$ & 28.16 & 28.40 & 28.65 & 28.89 \\
\hline 298.0 & 27.26 & 27.51 & 27.76 & 28.00 & 28.25 & 28.49 & 28.74 \\
\hline 300.0 & 27.11 & 27.36 & 27.61 & 27.85 & 28.10 & 28.34 & 28.38 \\
\hline 302.0 & 26.97 & 27.21 & 27.46 & 27.70 & 27.95 & 28.19 & 28.43 \\
\hline 304.0 & 26.82 & 27.07 & 27.31 & 27.56 & 27.80 & 28.04 & 28.28 \\
\hline 305.0 & 26.68 & 26.92 & 27.17 & 27.41 & 27.65 & 27.89 & 28.13 \\
\hline 308.0 & 26.54 & 0.6 .78 & 27.03 & 27.27 & 27.51 & 27.75 & 27.99 \\
\hline 310.0 & 26.40 & 26.64 & 26.88 & 27.13 & 27.37 & 27.60 & 27.84 \\
\hline $\begin{array}{l}312.0 \\
314.0\end{array}$ & 26.26 & $\begin{array}{l}26.50 \\
26.36\end{array}$ & $\begin{array}{l}26.74 \\
25.61\end{array}$ & $\begin{array}{l}26.98 \\
26.84\end{array}$ & $\begin{array}{l}27.22 \\
27.08\end{array}$ & $\begin{array}{l}27.46 \\
27.32\end{array}$ & $\begin{array}{l}27.70 \\
27.36\end{array}$ \\
\hline 316.0 & 25.99 & 26.23 & $\begin{array}{l}23.61 \\
26.47\end{array}$ & $\begin{array}{l}20.07 \\
26.71\end{array}$ & 26.94 & $\begin{array}{l}27.32 \\
27.18\end{array}$ & 27.42 \\
\hline 318.0 & 25.85 & 26.09 & 26.33 & 25.57 & 26.81 & 27.04 & 27.28 \\
\hline 320.0 & 25.72 & 25.96 & 20.20 & 26.44 & 26.67 & 26.91 & 27.14 \\
\hline 322.0 & 25.59 & 25.83 & 26.07 & 26.30 & 26.54 & 26.77 & 27.00 \\
\hline $\begin{array}{l}324.0 \\
326.0\end{array}$ & $\begin{array}{l}25.45 \\
25.33\end{array}$ & $\begin{array}{l}25.70 \\
25.57\end{array}$ & $\begin{array}{l}25.93 \\
25.80\end{array}$ & $\begin{array}{l}26.17 \\
25.04\end{array}$ & $\begin{array}{l}26.40 \\
26.27\end{array}$ & $\begin{array}{l}26.64 \\
26.50\end{array}$ & $\begin{array}{l}26.87 \\
26.74\end{array}$ \\
\hline $3 \geq 8.0$ & $25 . ? 1$ & 25.44 & 25.68 & 25.91 & 26.14 & 26.37 & 26.60 \\
\hline 330.0 & 25.08 & 25.32 & 25.55 & 25.78 & 26.01 & 26.24 & 26.47 \\
\hline 332.0 & 24.96 & 25.19 & 25.42 & 25.65 & 25.89 & 26.12 & 26.34 \\
\hline 334.0 & 24.83 & 25.07 & 25.30 & 25.53 & 25.76 & 25.99 & 26.22 \\
\hline $33 \% .0$ & $? 4.71$ & 24.94 & 25.17 & 25.41 & 25.63 & 25.86 & 26.09 \\
\hline 338.0 & 24.59 & 34.82 & 25.05 & & 25.51 & 25.74 & 25.96 \\
\hline $\begin{array}{l}340.0 \\
342.0\end{array}$ & 24.47 & 24.70 & 24.93 & 25.16 & 25.39 & 25.61 & 25.84 \\
\hline $\begin{array}{l}342.0 \\
344.0\end{array}$ & $\begin{array}{l}24.35 \\
24.24\end{array}$ & $\begin{array}{l}24.58 \\
24.47\end{array}$ & $\begin{array}{l}24.81 \\
24.69\end{array}$ & $\begin{array}{l}25.04 \\
24.02\end{array}$ & $\begin{array}{l}25.27 \\
25.15\end{array}$ & $\begin{array}{l}25.49 \\
25.37\end{array}$ & $\begin{array}{l}25.72 \\
25.59\end{array}$ \\
\hline $\begin{array}{l}544.0 \\
346.0\end{array}$ & 24.12 & 24.35 & $\begin{array}{l}24.09 \\
24.59\end{array}$ & $\begin{array}{l}24.92 \\
24.80\end{array}$ & $\begin{array}{l}25.15 \\
25.03\end{array}$ & $\begin{array}{l}25.37 \\
25.25\end{array}$ & $\begin{array}{l}25.39 \\
25.47\end{array}$ \\
\hline 348.0 & 24.01 & 24.23 & 24.46 & 24.68 & 24.91 & 25.13 & 25.33 \\
\hline 350.0 & 23.89 & 24.12 & 24.34 & 24.57 & 24.79 & 25.01 & 25.24 \\
\hline 352.0 & 23.78 & 24.01 & 24.23 & 24.45 & 24.68 & 24.90 & 25.12 \\
\hline 354.0 & 23.67 & 23.89 & 24.12 & 24.34 & 24.56 & 24.78 & 25.00 \\
\hline 355.0 & $\begin{array}{l}23.56 \\
23.45\end{array}$ & 23.78 & 24.01 & 24.23 & 24.45 & 24.67 & 24.89 \\
\hline $\begin{array}{l}358.0 \\
360.0\end{array}$ & $\begin{array}{l}23.45 \\
23.34\end{array}$ & $\begin{array}{l}23.57 \\
23.55\end{array}$ & $\begin{array}{l}23.89 \\
23.79\end{array}$ & $\begin{array}{l}24.12 \\
24.01\end{array}$ & $\begin{array}{l}24.34 \\
24.23\end{array}$ & $\begin{array}{l}24.56 \\
24.444\end{array}$ & $\begin{array}{l}24.77 \\
24.66\end{array}$ \\
\hline $\begin{array}{l}360.0 \\
36 ? .0\end{array}$ & $\begin{array}{l}23.34 \\
23.23\end{array}$ & $\begin{array}{l}230030 \\
23.46\end{array}$ & $\begin{array}{l}23.79 \\
23.58\end{array}$ & $\begin{array}{l}24.01 \\
23.90\end{array}$ & $\begin{array}{l}24.23 \\
24.12\end{array}$ & $\begin{array}{l}24.44 \\
24.33\end{array}$ & $\begin{array}{l}24.66 \\
24.55\end{array}$ \\
\hline 364.0 & 23.13 & 23.35 & $\begin{array}{r}23.57 \\
23.45\end{array}$ & 23.70 & 24.01 & 24.22 & 24.44 \\
\hline $\begin{array}{l}365.0 \\
368.0\end{array}$ & $\begin{array}{l}23.02 \\
? 2.92\end{array}$ & $\begin{array}{l}23.24 \\
23.14\end{array}$ & $\begin{array}{l}23.46 \\
23.36\end{array}$ & $\begin{array}{l}23.68 \\
23.57\end{array}$ & $\begin{array}{l}23.90 \\
23.79\end{array}$ & 24.11 & $\begin{array}{l}24.33 \\
24.22\end{array}$ \\
\hline 370.0 & 22.82 & 23.03 & $\begin{array}{l}23.36 \\
23.25\end{array}$ & 23.47 & $\begin{array}{l}23.74 \\
23.68\end{array}$ & $\begin{array}{l}24.01 \\
23.90\end{array}$ & 24.11 \\
\hline
\end{tabular}


HYDRDGEN DENSITY (KG/M3).

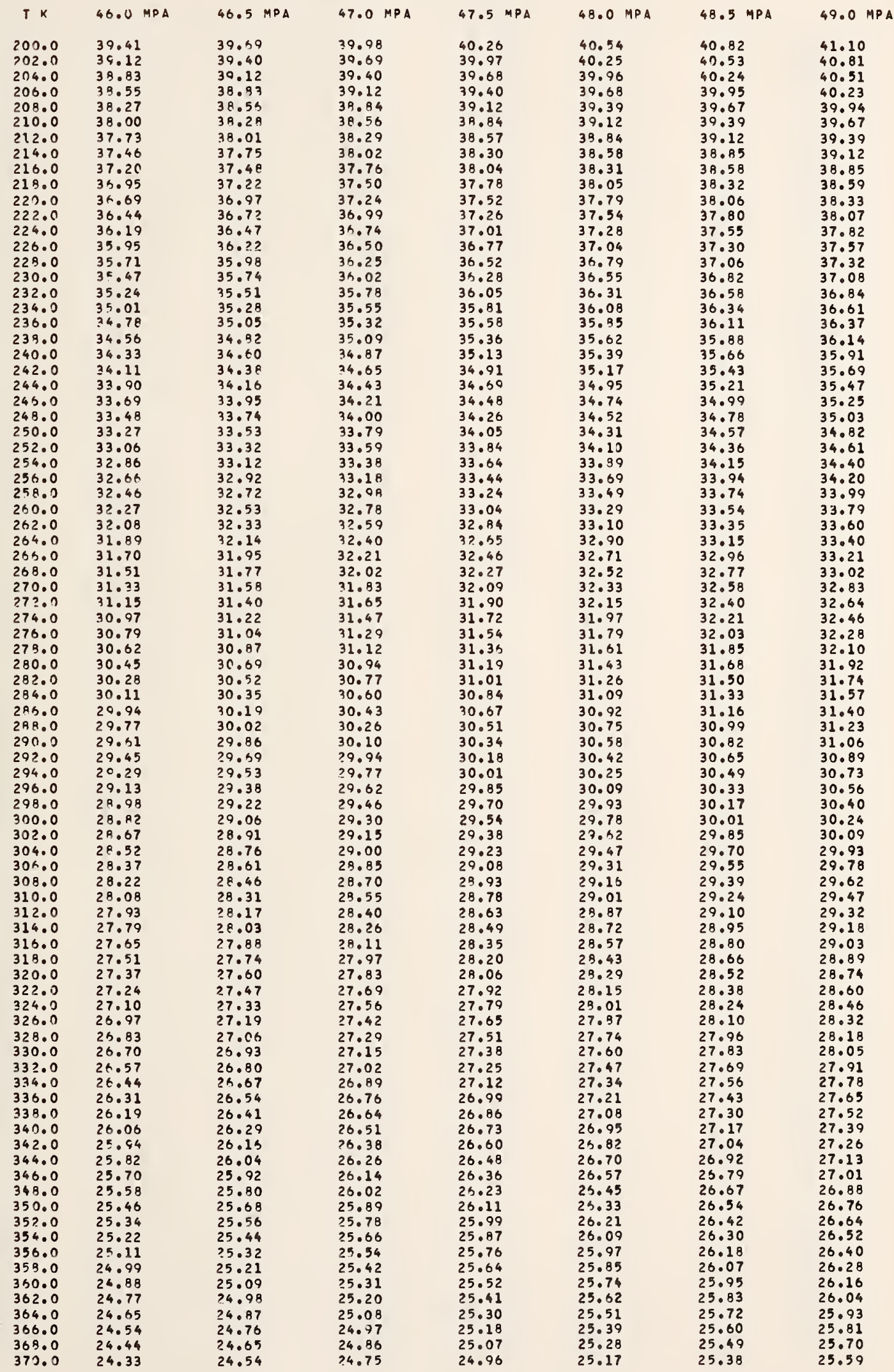


HYDROGEN OENSITY (KG/M3).

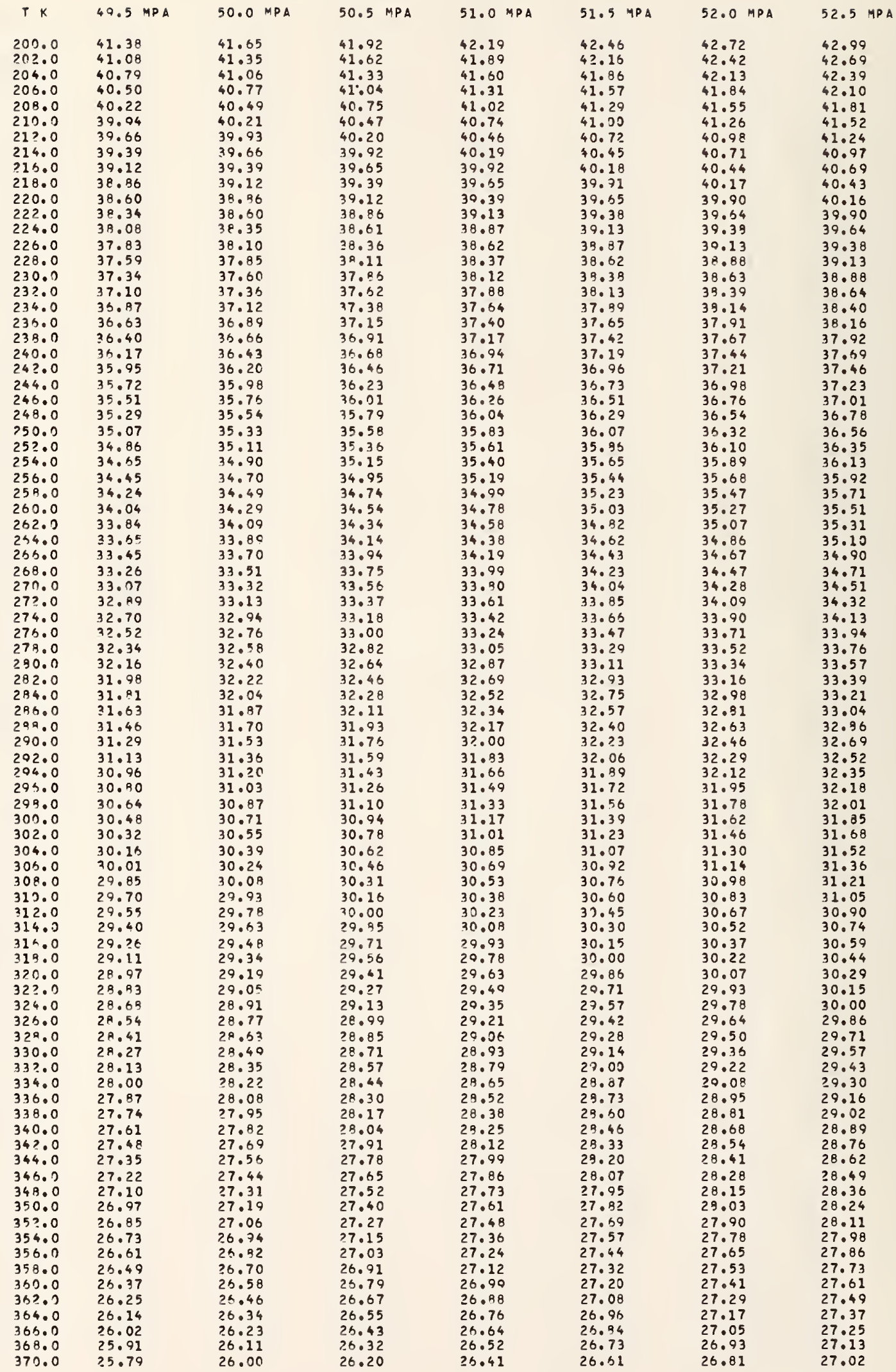


HYDROGEN DENSITY (KG/M3).

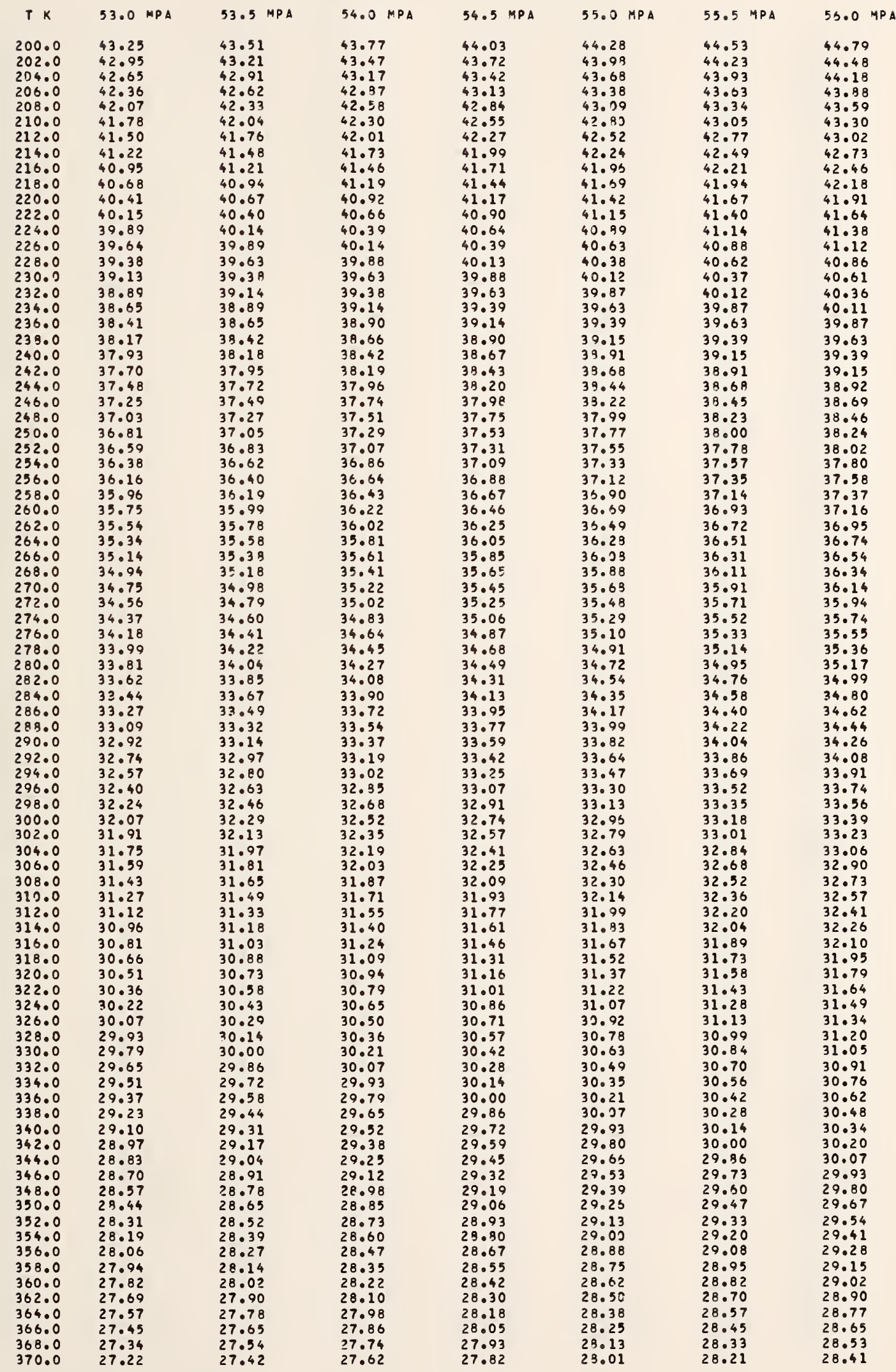


HYOROGEN DENSITY (KG/M3).

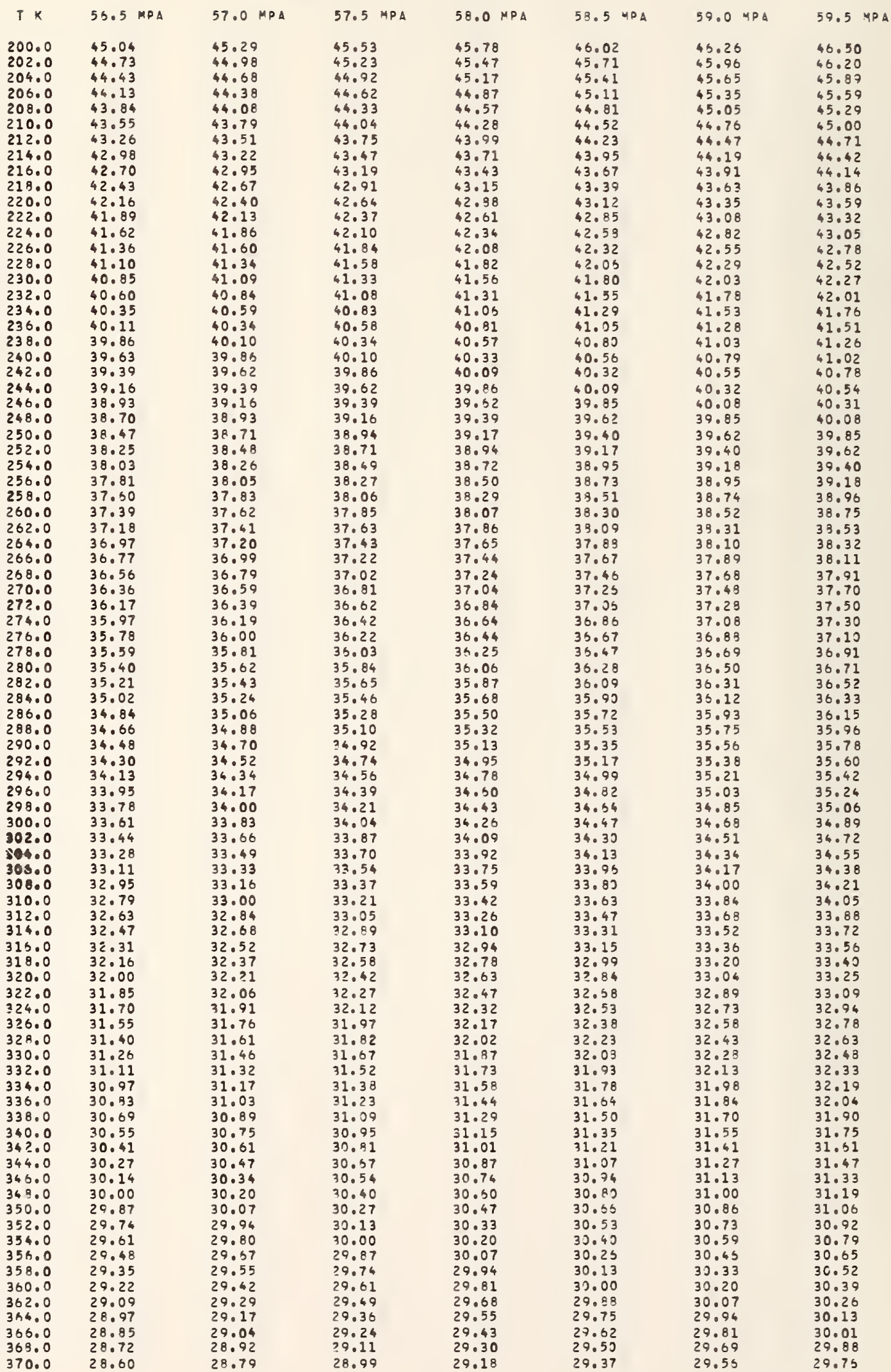


HYDROGEN DENSITY (KG/M3).

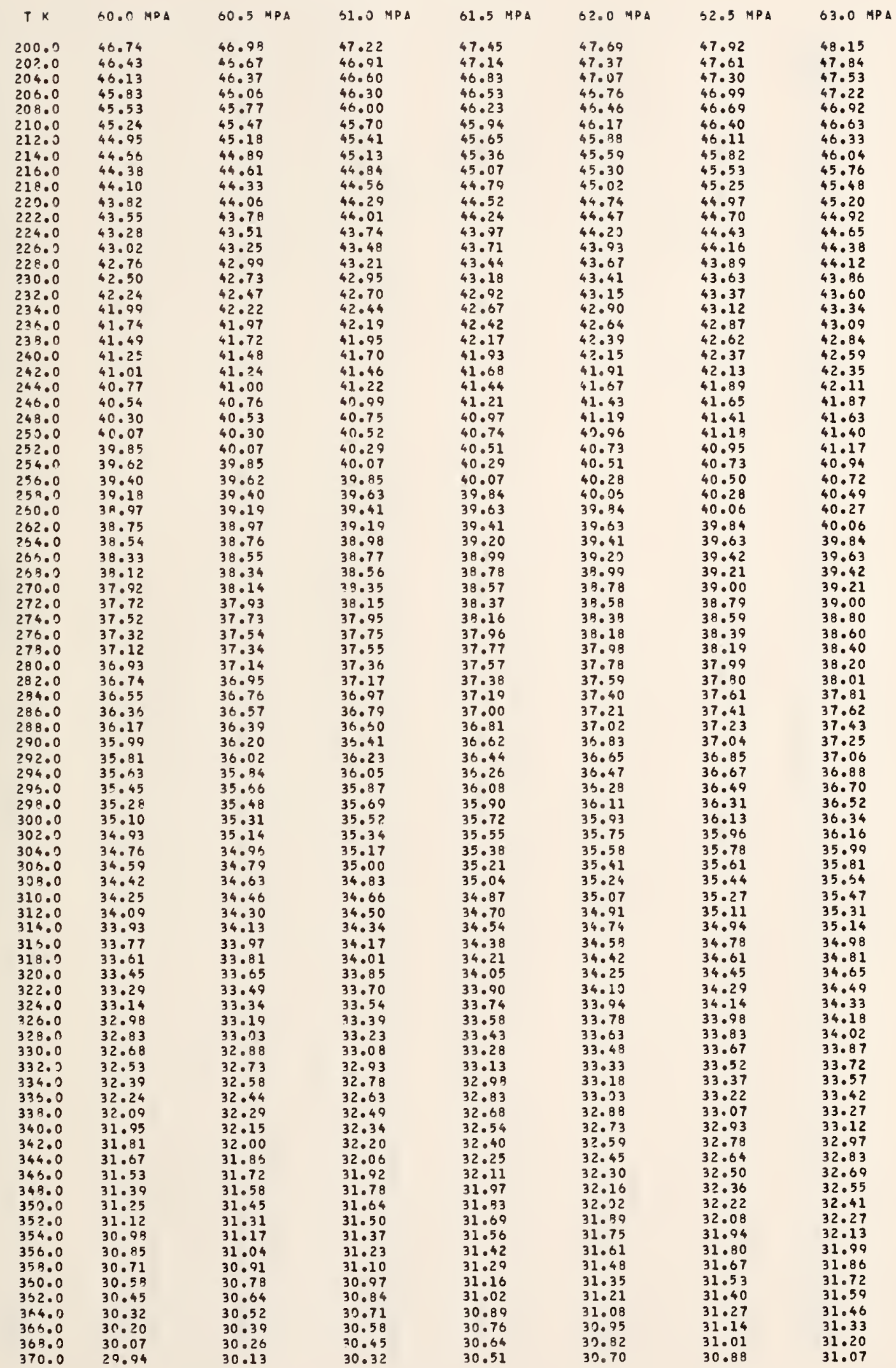


HYORDGEN DENSITY (KG/M3).

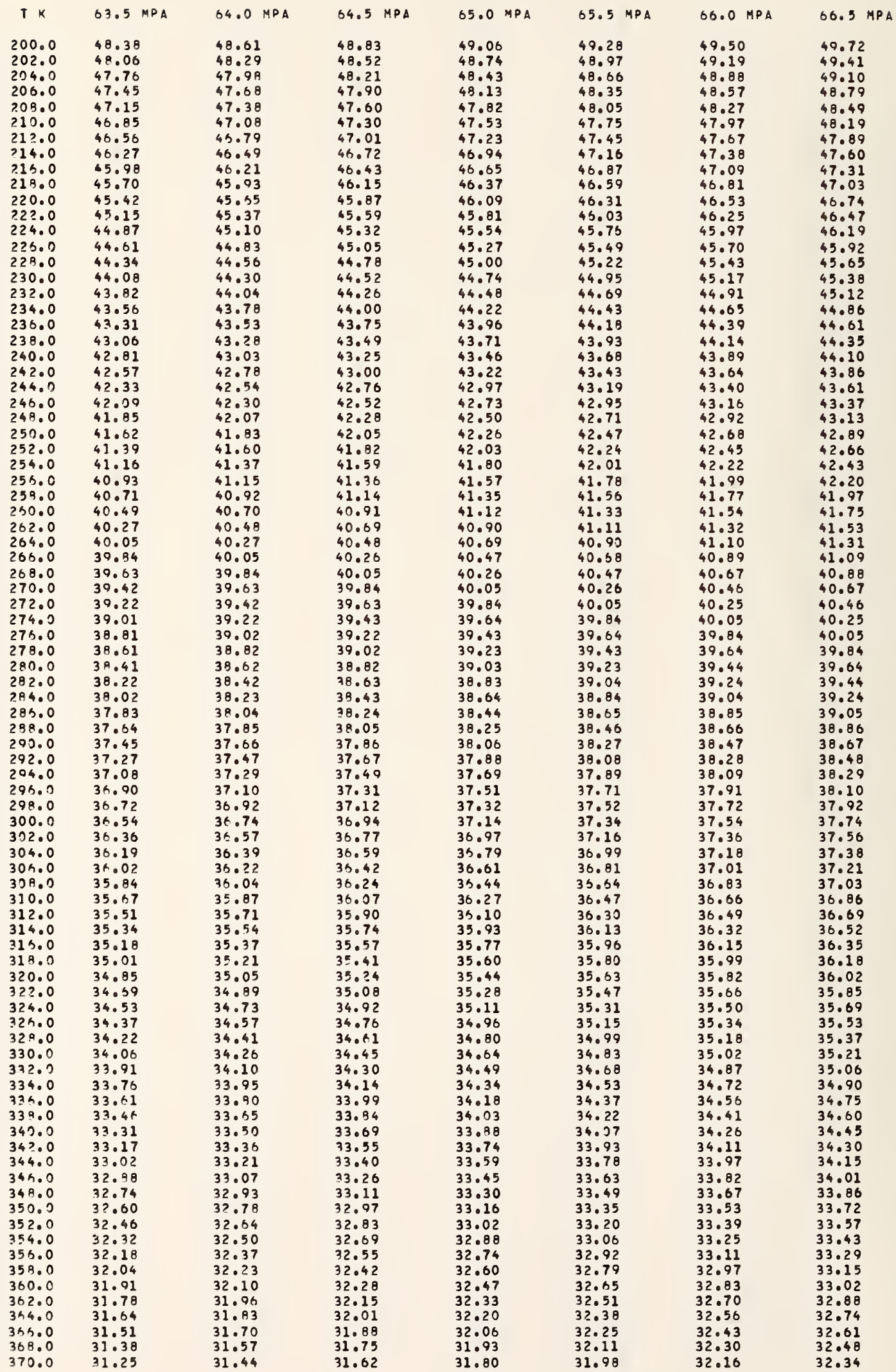


HYOROGEN DENSITY (KG/M3).

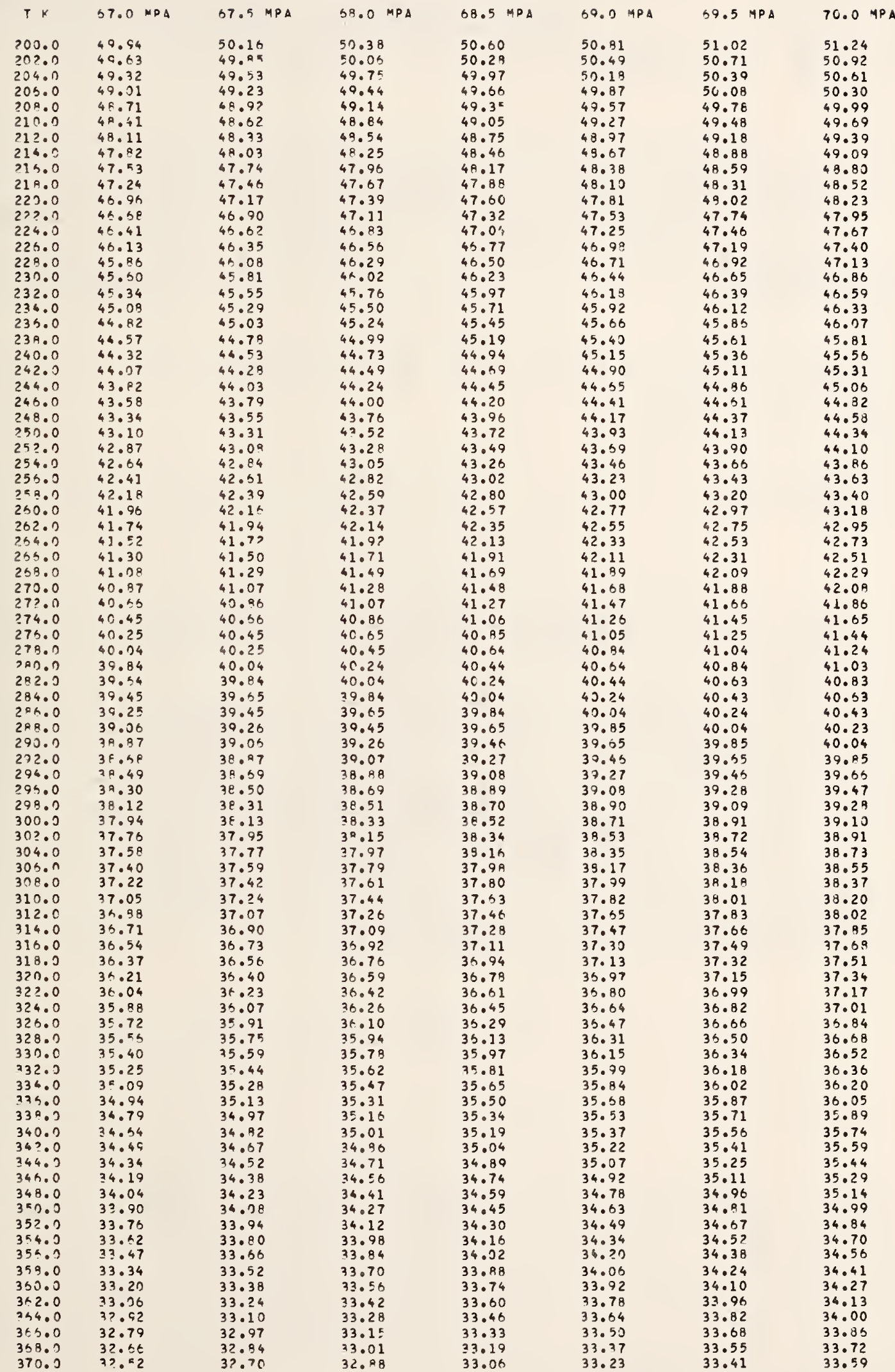


NBS-114A (REV. 2-BC)

U.S. DEPT. OF COMM.

BIBLIOGRAPHIC DATA

SHEET (See instructions)

1. PUBLICATION OR
REPORT NO.
NBS TN-1079

2. Performing Organ. Report No.

3. Publication $\overline{\text { Date }}$

NBS TN-1079

June 1985

4. TITLE AND SUBTITLE

TABLES OF INDUSTRIAL GAS CONTAINER CONTENTS AND DENSITY FOR OXYGEN, ARGON, NITROGEN, HELIUM, AND HYDROGEN

5. AUTHOR(S)

Ben A. Younglove and Nell A. Olien

6. PERFORMING ORGANIZATION (If joint or other than NBS, see in structions)

7. Contract/Grant No.

NATIONAL BUREAU OF STANDARDS

DEPARTMENT OF COMMERCE

WASHINGTON, D.C. 20234

8. Type of Report \& Period Covered

\section{SPONSORING ORGANIZATIOH NAMC AND COMPLETE ADDRESS (Street, City, Stote, ZIP)}

Compressed Gas Association

1235 Jefferson Davis Highway

Arlington, VA 22202

10. SUPPLEMENTARY NOTES

[.] Document describes a computer program; SF-185, FIPS Software Summary, is attached.

11. ABSTRACT (A 200-word or less factual summary of most significant information. If document includes a significant bibliography or literature survey. mention it here)

Custody transfer tables are presented for oxygen, argon, nitrogen, hellum, and hydrogen. The tables are based on standard reference data previously compiled by the National Bureau of Standards. Two sets of tables are provided for each fluid. Tables in engineering units cover the range -40 to $130^{\circ} \mathrm{F}$ with pressures from 100 to $10,000 \mathrm{psig}$. Tables in SI units (density versus pressure and temperature) cover the range 200 to $370 \mathrm{~K}$ with pressures from 0.5 to $70 \mathrm{MPa}$. The tables in engineering units are designed to provide a means of determining the volume of gas at standard conditions contained in a tank given the volume of the tank and the pressure and temperature of the gas within the tank. The publication also includes four examples of use of the tables in calculating tank quantities.

12. KEY WORDS (Six to twelve entries; alphabetical order; capitalize only proper names; and separate key words by semicolons) argon; custody transfer; gas density; gas volume; helium; hydrogen; nitrogen; oxygen

13. AVAILABILITY

Xulimited

$\square$ For Official Distribution. Do Not Release to NTIS

[X] Order From Superintendent of Documents, U.S. Government Printing Office, Washington, D.C. 20402.
14. NO. OF PRINTED PAGES

$$
200
$$

15. Price 


\section{Technical Publications}

\section{Periodicals}

Journal of Research-The Journal of Research of the National Bureau of Standards reports NBS research and development in those disciplines of the physical and engineering sciences in which the Bureau is active. These include physics, chemistry, engineering, mathematics, and computer sciences. Papers cover a broad range of subjects, with major emphasis on measurement methodology and the basic technology underlying standardization. Also included from time to time are survey articles on iopics closely related to the Bureau's technical and scientific programs. As a special service to subscribers each issue contains complete citations to all recent Bureau publications in both NBS and non-NBS media. Issued six times a year.

\section{Nonperiodicals}

Monographs-Major contributions to the technical literature on various subjects related to the Bureau's scientific and technical activities.

Handbooks-Recommended codes of engineering and industrial practice (including safety codes) developed in cooperation with interested industries, professional organizations, and regulatory bodies.

Special Publications-Include proceedings of conferences sponsored by NBS, NBS annual reports, and other special publications appropriate to this grouping such as wall charts, pocket cards, and bibliographies.

Applied Mathematics Series-Mathematical tables, manuals, and studies of special interest to physicists, engineers, chemists, biologists, mathematicians, computer programmers, and others engaged in scientific and technical work.

National Standard Reference I)ata Series-Provides quantitative data on the physical and chemical properties of materials, compiled from the world's literature and critically evaluated. Developed under a worldwide program coordinated by NBS under the authority of the National Standard Data Act (Public Law 90-396).

NOTE: The Journal of Physical and Chemical Reference Data (JPCRD) is published quarterly for NBS by the American Chemical Society (ACS) and the American Institute of Physics (AIP). Subscriptions, reprints, and supplements are available from ACS, 1155 Sixteenth St., NW, Washington, DC 20056.

Building Science Series-Disseminates technical information developed at the Bureau on building materials, components, systems, and whole structures. The series presents research results, test methods, and performance criteria related to the structural and environmental functions and the durability and safety characteristics of building elements and systems.

Technical Notes-Studies or reports which are complete in themselves but restrictive in their treatment of a subject. Analogous to monographs but not so comprehensive in scope or definitive in treatment of the subject area. Often serve as a vehicle for final reports of work performed at NBS under the sponsorship of other government agencies.

Voluntary Product Standards-Developed under procedures published by the Department of Commerce in Part 10, Title 15, of the Code of Federal Regulations. The standards establish nationally recognized requirements for products, and provide all concerned interests with a basis for common understanding of the characteristics of the products. NBS administers this program as a supplement to the activities of the private sector standardizing organizations.

Consumer Information Series-Practical information, based on NBS research and experience, covering areas of interest to the consumer. Easily understandable language and illustrations provide useful background knowledge for shopping in today's technological marketplace.

Order the abose NBS publications from: Superintendent of Documents, Government Printing Office,

Washington, DC 20402.

Order the following NBS publications-FIPS and NBSIR's-from the National Technical Information Service, Springfield, VA 22161.

Federal Information Processing Standards Publications (FIPS P( B)-Publications in this series collectively constitute the Federal Information Processing Standards Register. The Register serves as the official source of information in the Federal Government regarding standards issued by NBS pursuant to the Federal Property and Administrative Services Act of 1949 as amended, Public Lat 89-306 (79 Stat. 1127), and as implemented by Executive Order 11717 (38 FR 12315, dated May 11, 1973) and Part 6 of Title 15 CFR (Code of Federal Regulations).

NBS Interagency Reports (NBSIR)-A special series of interim or final reports on work performed by NBS for outside sponsors (both government and non-government). In general, initial distribution is handled by the sponsor; public distribution is by the National Technical Information Service, Springfield, VA 22161, in paper copy or microfiche form. 
U.S. Department of Commerce

National Bureau of Standards

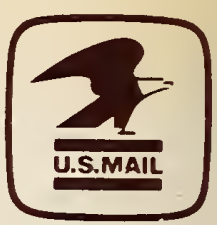

Washington, D.C. 20234

Official Business

Penalty for Private Use $\$ 300$

POSTAGE AND FEES PAID U.S DEPARTMENT OF COMMERCE COM-215

SPECIAL FOUATH-CLASS RATE

$$
\text { BOOK }
$$

